University of Rhode Island

DigitalCommons@URI

Open Access Dissertations

2012

\title{
NOVEL COMPOSITE MATERIALS AND SANDWICH STRUCTURES FOR BLAST MITIGATION
}

Nathaniel W, Gardner

University of Rhode Island, nate.gardner11@gmail.com

Follow this and additional works at: https://digitalcommons.uri.edu/oa_diss

\section{Recommended Citation}

Gardner, Nathaniel W, "NOVEL COMPOSITE MATERIALS AND SANDWICH STRUCTURES FOR BLAST MITIGATION" (2012). Open Access Dissertations. Paper 94.

https://digitalcommons.uri.edu/oa_diss/94

This Dissertation is brought to you for free and open access by DigitalCommons@URI. It has been accepted for inclusion in Open Access Dissertations by an authorized administrator of DigitalCommons@URI. For more information, please contact digitalcommons-group@uri.edu. 


\section{NOVEL COMPOSITE MATERIALS AND \\ SANDWICH STRUCTURES FOR BLAST \\ MITIGATION}

BY

NATHANIEL W. GARDNER

A DISSERTATION SUBMITTED IN PARTIAL FULFILLMENT OF THE

REQUIREMENTS FOR THE DEGREE OF

DOCTOR OF PHILOSOPHY

IN

MECHANICAL, INDUSTRIAL AND SYSTEMS ENGINEERING

UNIVERSITY OF RHODE ISLAND 


\title{
DOCTOR OF PHILOSOPHY DISSERTATION
}

\section{BY}

NATHANIEL W. GARDNER

\section{APPROVED:}

Thesis Committee:

\author{
Major Professor Dr. Arun Shukla \\ Dr. Martin H. Sadd \\ Dr. David G. Taggart \\ Dr. Michael L. Greenfield \\ Nasser H. Zawia \\ DEAN OF THE GRADUATE SCHOOL
}

UNIVERSITY OF RHODE ISLAND 


\begin{abstract}
An experimental study has been conducted to investigate the blast resistance and mitigation behaviors of novel composites and sandwich structures. Understanding the overall behaviors and failure mechanisms will aid in the development of optimally designed light-weight structures that can mitigate energy and maintain structural integrity when subjected to blast loadings. Due to the increased threat of damage to civilian and defense structures in the form of terrorist attacks and blast loading, a comprehensive understanding on blast mitigation of composites and sandwich structures, as well as an optimal design, is essential.

The dynamic behavior of various sandwich composites made of E-glass VinylEster (EVE) facesheets and Corecell ${ }^{\mathrm{TM}}$ A-series styrene acrylonitrile (SAN) foam core was studied using a shock tube apparatus. The overall specimen dimensions were held constant for all core configurations studied, more specifically the foam core thickness. Prior to shock tube testing, the quasi-static and dynamic constitutive behavior of the facesheets (tensile/compressive) and foam (compressive) was evaluated. During the shock tube testing, a high-speed photography system was utilized to capture the real-time deformation process, as well as mechanisms of failure. In the later studies, high-speed photography was coupled with the optical technique of 3-D Digital Image Correlation (DIC) to obtain the real-time, full-field deformation process, including the out-of-plane deflection and velocity, as well as in-plane strain. Post-mortem analysis was also carried out to evaluate the overall blast performance of these configurations.
\end{abstract}


First, shock tube experiments were performed to study the dynamic response of sandwich panels with E-glass Vinyl-Ester (EVE) composite facesheets and stepwise graded styrene acrylonitrile (SAN) foam cores. Two types of core configurations, with identical areal density, were subjected to the shock wave loading. The core layers were arranged according to the density of the respective foam; configuration 1 consisted of low / middle / high density foams and configuration 2 consisted of middle / low / high density foams. The method to calculate the incident and reflected energies of the shock wave, as well as the deformation energy of the specimen, were proposed based on the shock wave pressure profiles and the high-speed deflection images that were obtained. The experimental results showed that configuration 1 outperformed configuration 2 in regards to their blast resistance. Significant core material compression was observed in configuration 1, while in configuration 2 the core layers disintegrated and the front skin (blast side) fractured into two pieces along the midsection. The foam core compression in configuration 1 reduced the dynamic pressures seen on the back facesheet, and thus limited the total amount of damage imparted on the specimen. The estimated energies were then calculated for both configurations. The total energy difference between the incident and reflected energies was almost identical, even though the deformation energy for configuration 2 was larger.

Since it was observed that a stepwise graded foam core allows for more compression in the core, thus reducing dynamic pressures seen on the back facesheet, and limiting the total amount of damage imparted on the specimen, the study was then continued to investigate the influence of the number of foam core layers, as well as 
material interfaces, on the dynamic response of sandwich structures. Four types of core configurations were subjected to the shock wave loading. The foam core was monotonically graded based on increasing acoustic wave impedance, with the foam core layer of lowest wave impedance facing the blast. The specimen dimensions were held constant for all core configurations, while the number of core layers varied, resulting in specimens with one layer, two layer, three layer, and four layers of foam core gradation. The results indicated that even though each configuration allowed for a stepwise compression of the core, the number of core layers has an influence on the dynamic response of the structure under blast loading. More specifically, by increasing the number of monotonically graded layers, the acoustic wave impedance mismatch between successive layers is reduced. Therefore, the strength of the initial shock wave (stress wave) can be weakened by the time it reaches the back facesheet, resulting in lower back face deflection, in-plane strain, and velocity. More importantly, the overall damage imparted on the structure can be reduced and structural integrity can be maintained.

Due to the fact that higher levels of core gradation helped maintain structural integrity and improved the overall blast performance of sandwich structures, the study was then continued to investigate the blast response of sandwich structures with a functionally graded core and polyurea (PU) interlayer, and more importantly how the location of this polyurea interlayer affects the overall behavior and blast performance. Two types of core configurations were subjected to shock wave loading. The materials, as well as the core layer arrangements, were identical, with the only difference arising in the location of the polyurea interlayer. The foam core itself was 
layered with monotonically increasing wave impedance of the core layers, with the lowest wave impedance facing the shock loading. For configuration 1, the polyurea interlayer was placed behind the front facesheet, in front of the foam core, while in configuration 2 it was placed behind the foam core, in front of the back facesheet. The results indicated that applying polyurea behind the foam core and in front of the back facesheet will reduce the back face deflection, particle velocity, and in-plane strain, thus improving the overall blast performance and maintaining structural integrity.

Since an optimized core configuration was determined, the study was continued to investigate the relationship between the weight of the panel and its overall blast performance. Two types of core configuration were subjected to shock wave loading. The materials, as well as the core layer arrangements, and overall specimen dimensions were identical, with the only difference appearing in the core layers; one configuration utilized equivalent core layer thickness, while the other configuration utilized equivalent core layer mass. The foam core itself was layered based on monotonically increasing the acoustic wave impedance of the core layers, with the lowest wave impedance facing the shock loading. The results indicated that with a decrease in areal density of $\sim 1 \mathrm{~kg} / \mathrm{m}^{2}(5 \%)$ from the sandwich composites with equivalent core layer thickness to the sandwich composites with equivalent core layer mass, an increase in deflection (20\%), in-plain strain (8\%) and velocity (8\%) was observed.

Finally, since an optimal core configuration was developed to better mitigate blast loadings, and an in-depth study was performed on the relationship between the weight of the panel and its overall blast performance, the research was continued with 
composite facesheet designs to better mitigate impact and blast loadings. Two types of core configurations were subjected to shock wave loading. The core material and thickness, as well as overall specimen dimensions were held constant, with the only difference arising in the resin system used during the infusion. The non-core-shell rubber toughened resin system (Non-CSR) consisted of a Vinyl-Ester resin only; while the CSR toughened resin consisted of the same Vinyl-Ester resin, but with Kane Ace MX-153 nano-scale core-shell rubber particles added to the mixture. Results indicated that adding nano-scale core-shell rubber (CSR) particles to sandwich composites, aids in dispersing the initial shock wave loading, thus reducing the overall deflection, strain, and velocity and improving the overall blast resistance of the structure. 


\section{ACKNOWLEDGMENTS}

First and foremost, I would like to thank Dr. Arun Shukla for his continuous guidance and support throughout this research. His patience and adamant work ethic have truly been inspiring and motivating throughout my doctoral research. I cannot thank him enough for all of the positive things he has instilled into me to help me grow as an engineer, a researcher and most of all an individual. He is not only an outstanding professor and mentor, but a truly inspirational human being. It has been an honor for me to be one of his Ph.D. students.

I would also like to sincerely thank Dr. Martin H. Sadd, Dr. David G. Taggart, Dr. Michael L. Greenfield, Dr. Richard Brown and Dr. Philip Datseris for agreeing to serve as my committee members. A special thanks to Dr. Sadd for his critical discussion in the weekly group meetings and presentations.

The help and encouragement from my friends and colleagues are greatly appreciated. I would like to thank all of my lab mates in the Dynamic Photomechanics Laboratory that have been by my side and influenced me along the way: Addis Kidane, Mathew Jackson, Erheng Wang, Puneet Kumar, James LeBlanc, Sandeep Abotula, Sachin Gupta, Nicholas Heeder, Ryan Sekac, Jefferson Wright, Daniel Gracia, and Alexander Escher. A special thanks to Gifford W. Plume IV for contributing additional creativity and engineering knowledge when it came to our graduate coursework and individual research. Without him, my undergraduate and graduate studies at the University of Rhode Island would have been more difficult. The time spent with everyone has made it an incredible experience, as well as provided me with many great friendships. In addition, I would like to thank Joe 
Gomez, Dave Ferriera, Jim Byrnes, Rob D'Ambrosca, Jen Cerullo, Nancy Dubee, Sally Marinelli, Brittany Mathews and the rest of the mechanical engineering department faculty and staff.

I would like to acknowledge the financial support provided by Dr. Yapa D. S. Rajapakse under the Office of Naval Research (ONR) Grant No. N00014-10-1-0662 and Grant No. N00014-04-1-0268, as well as the financial support provided by the Department of Homeland Security (DHS) under Cooperative Agreement No. 2008ST-061-ED0002. I would also like to thank Gurit SP Technology (Matthew Bodoff) and Specialty Products, Inc. (Shere Bush) for providing the material, as well as Dr. Stephen Nolet and TPI Composites for providing the facility for creating the composites and sandwich structures used in these investigations. A special thanks to Dr. Richard Brown for providing the Scanning Electron Microscope (SEM) facility used in obtaining the images of the cell microstructures.

Last but not least, I would like to thank my parents Randy and Sandi, my brothers Graham and Ryan, my grandmother Catherine Waradzin and the rest of my extended family for their understanding nature and endless support throughout my studies. A special thank you to my dog Indyka for her undevoted love no matter what time I came home, and for providing me with an excuse to stop working and enjoy a walk. 


\section{PREFACE}

An experimental study has been conducted to investigate the blast resistance and mitigation behaviors of novel composites and sandwich structures. Understanding the overall behaviors and failure mechanisms will lead to optimally designed light-weight structures that can mitigate energy and maintain structural integrity when subjected to blast loadings. Due to the increased threat of damage to civilian and defense structures in the form of terrorist attacks and blast loading, a comprehensive understanding on blast mitigation of composites and sandwich structures, as well as an optimal design, is pivotal. This dissertation addresses the dynamic behaviors and the failure mechanisms of composite materials and sandwich structures under high-intensity airblast (shock wave) loading. This dissertation is prepared using the manuscript format.

Chapter 1 provides an overview of previous and current published literature of subject matter relevant to this dissertation. Topics include a brief background on sandwich composites, the use of sandwich composites as structural materials, and why they are important in the naval, aerospace and defense industry, as well as the idea of various core materials/structures, and functionally graded materials. This chapter serves to provide an overview of the relevant research in literature, the possible data gaps that exist, as well as an introduction to the studies within this dissertation.

Chapter 2 focuses on a controlled study performed to understand the effect of core gradation on the blast response of sandwich composites made of E-glass Vinyl-Ester (EVE) facesheets and a stepwise graded Corecell A-series foam core. A shock tube apparatus was utilized to generate a controllable and repeatable dynamic loading. Two types of core configurations, with identical areal density, were subjected to the air- 
blasts. The core layers were arranged according to the density of the respective foam; configuration 1 consisted of low / middle / high density foams and configuration 2 consisted of middle / low / high density foams. The method to calculate the incident and reflected energies of the shock wave, as well as the deformation energy of the specimen, were proposed based on the shock wave pressure profiles and the highspeed deflection images that were obtained. This chapter follows the formatting guidelines specified by the International Journal of Solids and Structures.

Chapter 3 details the experimental studies conducted to understand the effect of the number of functionally graded foam core layers, as well the effect of material interfaces, on the blast response of sandwich composites made of E-glass Vinyl-Ester (EVE) facesheets and graded Corecell ${ }^{\mathrm{TM}}$ A-series foam. The foam core was monotonically graded based on increasing acoustic wave impedance, with the foam core layer of lowest wave impedance facing the blast. A shock tube apparatus was utilized to generate a controllable and repeatable dynamic loading. Four core configurations were designed and fabricated. The specimen dimensions were held constant for all core configurations, while the number of core layers varied, resulting in specimens with one layer, two layer, three layer, and four layers of foam core gradation. Prior to shock tube testing, the quasi-static and dynamic constitutive behavior (compressive) of each type of foam was evaluated. During the shock tube testing, a high-speed photography coupled with the optical technique of Digital Image Correlation (DIC) was utilized to capture the real-time deformation process as well as mechanisms of failure. Post-mortem analysis was also carried out to evaluate the 
overall blast performance of these configurations. This chapter follows the formatting guidelines specified by Composite Structures.

Chapter 4 concentrates on the experimental studies conducted to understand the effect of polyurea, and its location, on the blast response of sandwich composites made of E-glass Vinyl-Ester (EVE) facesheets and a core made of Corecell ${ }^{\mathrm{TM}} \mathrm{A}$-series foam with a polyurea interlayer. A shock tube apparatus was utilized to generate a controllable and repeatable dynamic loading. Two types of core configurations were designed and fabricated. The materials, as well as the core layer arrangements, were identical, with the only difference arising in the location of the polyurea interlayer. The foam core itself was layered with monotonically increasing wave impedance of the core layers, with the lowest wave impedance facing the shock loading. For configuration 1 , the polyurea interlayer was placed behind the front facesheet, in front of the foam core, while in configuration 2 it was placed behind the foam core, in front of the back facesheet. A high-speed side-view camera, along with a high-speed backview 3-D Digital Image Correlation (DIC) system, was utilized to capture the realtime deformation process as well as mechanisms of failure. Post-mortem analysis was also carried out to evaluate the overall blast performance of these two configurations. This chapter follows the formatting guidelines specified by Experimental Mechanics.

Chapter 5 details the experimental studies conducted to understand the effect of equivalent core layer thickness or equivalent core layer mass on the blast response of sandwich composites made of E-glass Vinyl-Ester (EVE) facesheets and Corecell ${ }^{\mathrm{TM}}$ A-series foam. A shock tube apparatus was utilized to generate a controllable and repeatable dynamic loading. Two different core configurations were designed and 
fabricated. The materials, as well as the core layer arrangements, and overall specimen dimensions were identical, with the only difference appearing in the core layers; one configuration utilized equivalent core layer thickness, while the other configuration utilized equivalent core layer mass. The foam core itself was layered based on monotonically increasing the acoustic wave impedance of the core layers, with the lowest wave impedance facing the shock loading. During the shock tube testing, a high-speed photography system coupled with the optical technique of 3-D Digital Image Correlation (DIC) was utilized to capture the real-time deformation process as well as mechanisms of failure. Post-mortem analysis was carried out to evaluate the overall blast performance of these two configurations. This chapter will follow the formatting guidelines specified by Composites: Part B.

Chapter 6 focuses on the experimental studies conducted to understand the effect of nano-scale core-shell rubber (CSR) toughening on the dynamic behavior of sandwich composites made of E-glass Vinyl-Ester (EVE) facesheets and Corecell ${ }^{\mathrm{TM}}$ A-series foam. A shock tube apparatus was utilized to generate a controllable and repeatable dynamic loading. Two different core configurations were designed and fabricated. The core material and thickness, as well as overall specimen dimensions were held constant, with the only difference arising in the resin system used during the infusion. The non-core-shell rubber toughened resin system (Non-CSR) consisted of a vinyl-ester resin only; while the CSR toughened resin consisted of the same vinylester resin, but with Kane Ace MX-153 nano-scale core-shell rubber particles added to the mixture. Prior to shock tube testing, the quasi-static and dynamic constitutive behavior of the facesheets (tensile/compressive) and foam (compressive) was 
evaluated. During the shock tube testing, high-speed photography coupled with the optical technique of Digital Image Correlation (DIC) was utilized to capture the realtime deformation process as well as mechanisms of failure. Post-mortem analysis was also carried out to evaluate the overall blast performance of these configurations. This chapter will follow the formatting guidelines specified by the International Journal of Impact Engineering.

Chapter 7 provides a summary of the major experimental findings obtained during the investigation of novel composites and sandwich structures to mitigate blast loadings. Suggestions for future core designs, as well as experiments and analysis will also be provided. 


\section{TABLE OF CONTENTS}

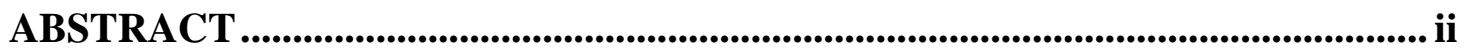

ACKNOWLEDGMENTS ....................................................................................................... vii

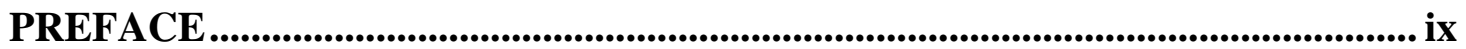

TABLE OF CONTENTS.......................................................................................... xiv

LIST OF TABLES …................................................................................................... xxi

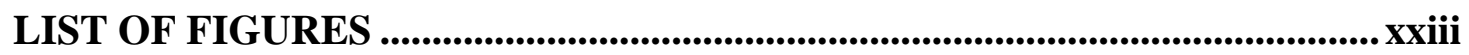

CHAPTER 1: INTRODUCTION AND LITERATURE REVIEW ......................... 1

CHAPTER 2: THE BLAST RESISTANCE OF SANDWICH COMPOSITES

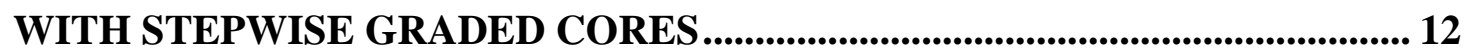

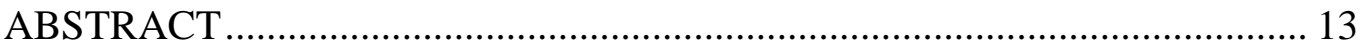

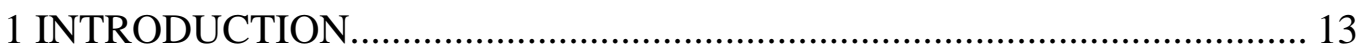

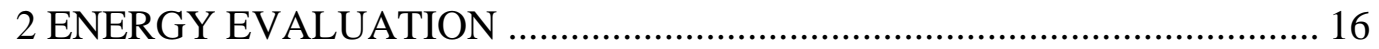

2.1 THE INCIDENT AND REFLECTED ENERGIES ............................... 17

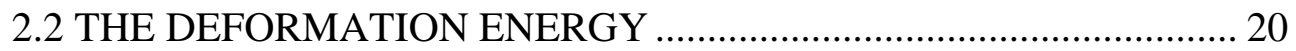

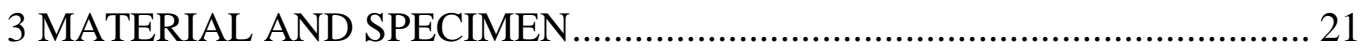

3.1 SKIN AND CORE MATERIALS ………………………………...... 21

3.2 SANDWICH PANELS WITH STEWPISE GRADED CORE LAYER

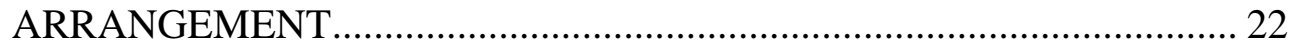

4 EXPERIMENTAL SET-UP AND PROCEDURE ......................................... 23

4.1 MODIFIED SPLIT HOPKINSON PRESSURE BAR WITH HOLLOW

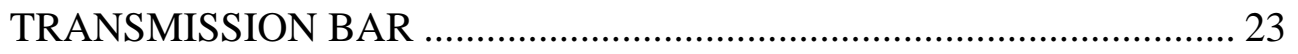

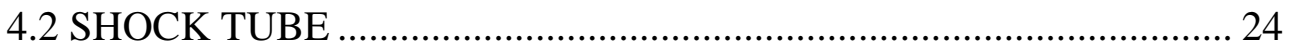


4.3 EXPERIMENTAL PROCEDURE AND PARAMETERS 25

5 EXPERIMENTAL RESULTS AND DISCUSSION ................................. 27

5.1 DYNAMIC BEHAVIOR OR CORE MATERIALS ........................ 27

5.2 BLAST RESPONSE OF SANDWICH COMPOSITES WITH

STEPWISE GRADED CORE ............................................................ 28

5.2.1 REAL-TIME DEFORMATION .......................................... 28

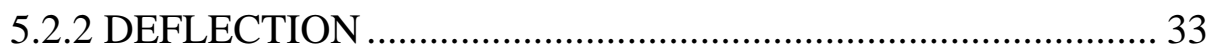

5.2.3 POST-MORTEM ANALYSIS .......................................... 34

5.2.3 ENERGY EVALUATION................................................... 36

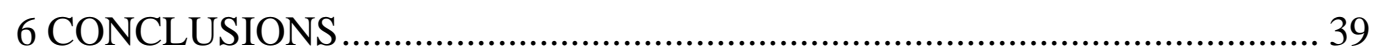

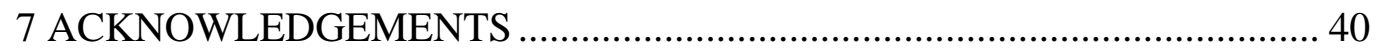

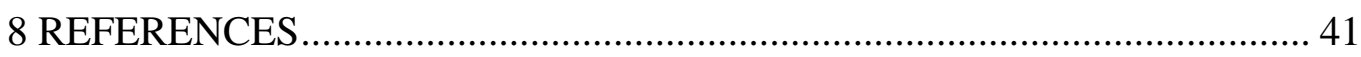

CHAPTER 3: PERFORMANCE OF FUNCTIONALLY GRADED SANDWICH COMPOSITE BEAMS UNDER SHOCK WAVE LOADING ............................. 43

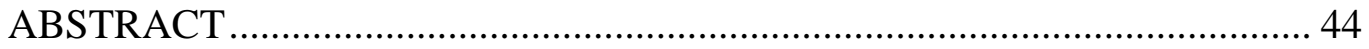

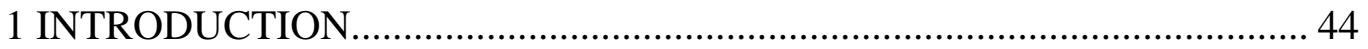

2 MATERIAL AND SPECIMEN ........................................................... 48

2.1 SKIN AND CORE MATERIALS ................................................. 48

2.2 SANDWICH PANELS WITH FUNCTIONALLY GRADED CORE

LAYER ARRANGMENT............................................................. 51

3 EXPERIMENTAL SET-UP AND PROCEDURE ...................................... 53

3.1 MODIFIED SPLIT HOPKINSON PRESSURE BAR WITH HOLLOW

TRANSMISSION BAR ............................................................... 53 
3.3 HIGH-SPEED PHOTOGRAPHY SYSTEM ..................................... 55

3.4 EXPERIMENTAL PROCEDURE AND PARAMETERS.................... 56

4 EXPERIMENTAL RESULTS AND DISCUSSION ....................................... 57

4.1 DYNAMIC BEHAVIOR OF CORE MATERIALS ............................ 57

4.2 BLAST RESPONSE OF SANDWICH COMPOSITES WITH

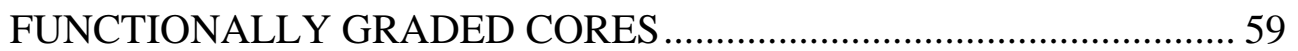

4.2.1 REAL-TIME DEFORMATION .............................................. 59

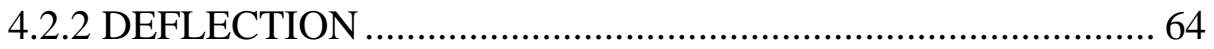

4.2.3 DIGITAL IMAGE CORRELATION (DIC) ANALYSIS ........... 73

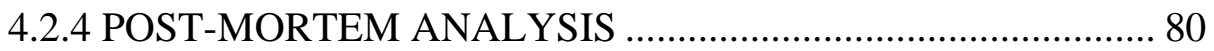

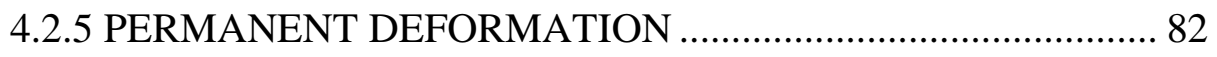

5 DISCUSSION ON STRESS WAVE PROPAGATION IN FUNCTIONALLY

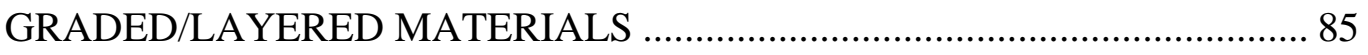

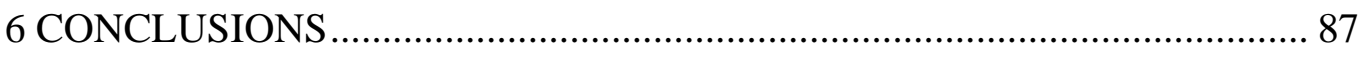

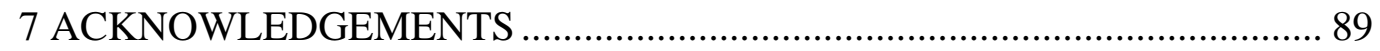

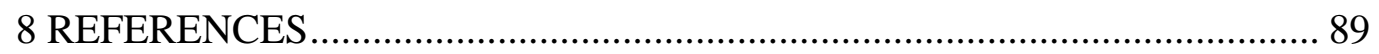

CHAPTER 4: BLAST MITIGATION IN A SANDWICH COMPOSITE USING

A GRADED CORE AND POLYUREA INTERLAYER .......................................... 93

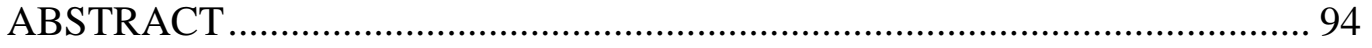

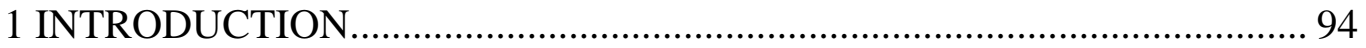

2 MATERIAL AND SPECIMEN.................................................................. 97

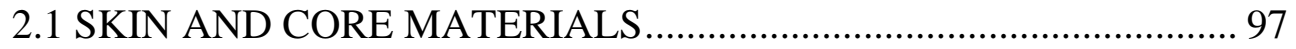


2.2 SANDWICH PANELS WITH FUNCTIONALLY GRADED CORE

LAYER ARRANGEMENT AND POLYUREA (PU) INTERLAYER ..... 99

3 EXPERIMENTAL SET-UP AND PROCEDURE …………………............ 100

3.1 MODIFIED SPLIT HOPKINSON PRESSURE BAR WITH HOLLOW

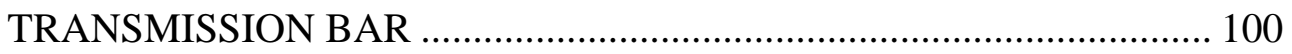

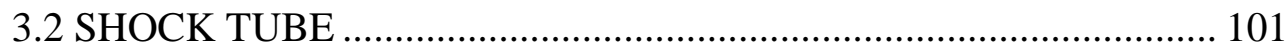

3.3 HIGH-SPEED PHOTOGRAPHY SYSTEM .................................... 102

3.4 EXPERIMENTAL PROCEDURE AND PARAMETERS.................. 103

4 EXPERIMENTAL RESULTS AND DISCUSSION .................................... 105

4.1 DYNAMIC BEHAVIOR OF CORE MATERIALS ............................ 105

4.2 BLAST RESPONSE OF SANDWICH COMPOSITES WITH

FUNCTIONALLY GRADED CORE AND PU INTERLAYER .............. 106

4.2.1 REAL-TIME DEFORMATION .............................................. 106

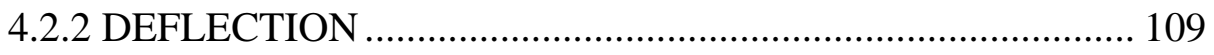

4.2.3 DIGITAL IMAGE CORRELATION (DIC) ANALYSIS ......... 115

4.2.4 POST-MORTEM ANALYSIS ............................................... 119

4.2.5 ENERGY REDISTRIBUTION BEHAVIOR........................... 122

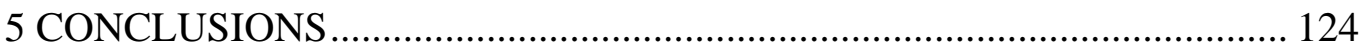

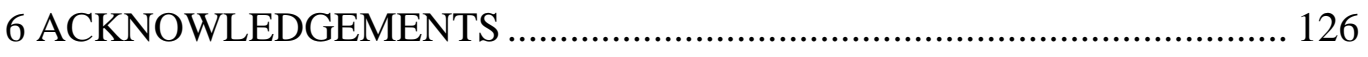

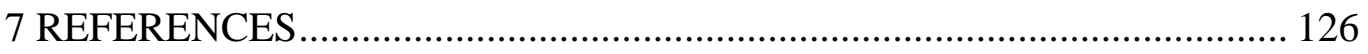

CHAPTER 5: THE BLAST RESPONSE OF SANDWICH COMPOSITES

WITH GRADED CORE: EQUIVALENT CORE LAYER THICKNESS VS

EQUIVALENT CORE LAYER MASS ........................................................................... 129 
ABSTRACT 130

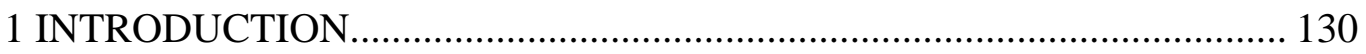

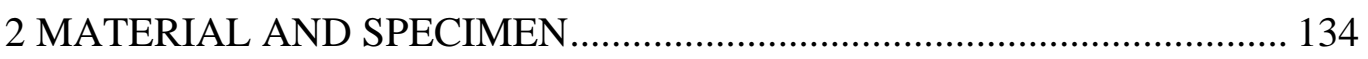

2.1 SKIN (FACESHEET) AND CORE MATERIALS ............................. 134

2.2 SANDWICH PANELS WITH EQ. CORE LAYER THICKNESS AND

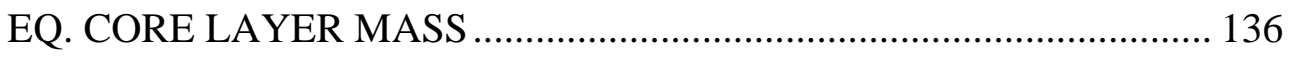

3 EXPERIMENTAL SET-UP AND PROCEDURE ......................................... 139

3.1 MODIFIED SPLIT HOPKINSON PRESSURE BAR WITH HOLLOW

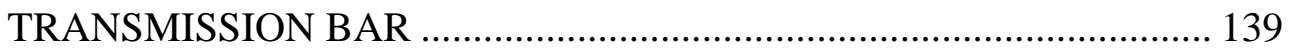

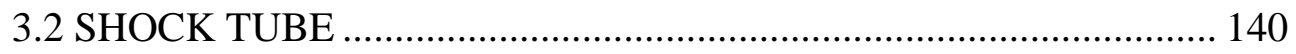

3.3 HIGH-SPEED PHOTOGRAPHY SYSTEM ..................................... 143

3.4 EXPERIMENTAL PROCEDURE AND PARAMETERS.................. 144

4 EXPERIMENTAL RESULTS AND DISCUSSION .................................... 145

4.1 DYNAMIC BEHAVIOR OF CORE MATERIALS ............................ 145

4.2 BLAST RESPONSE OF SANDWICH COMPOSITES WITH EQ.

CORE LAYER THICKNESS AND EQ. CORE LAYER MASS ............ 147

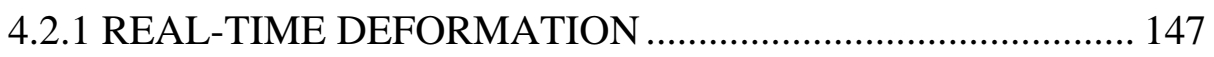

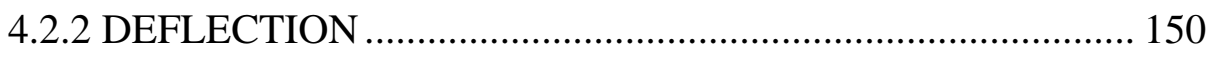

4.2.3 DIGITAL IMAGE CORRELATION (DIC) ANALYSIS ........ 153

4.2.4 POST-MORTEM ANALYSIS ............................................... 159

4.2.5 ENERGY REDISTRIBUTION BEHAVIOR ............................ 160

4.2.6 BENDING STIFFNESS AND STRENGTH OF SANDWICH

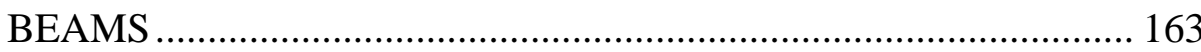


5 CONCLUSIONS 169

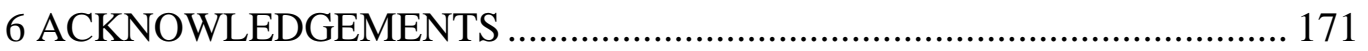

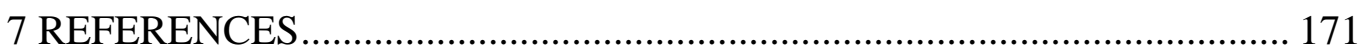

CHAPTER 6: THE BLAST RESISTANCE OF NANO-SCALE CORE-SHELL RUBBER (CSR) TOUGHENED SANDWICH COMPOSITES .......................... 175

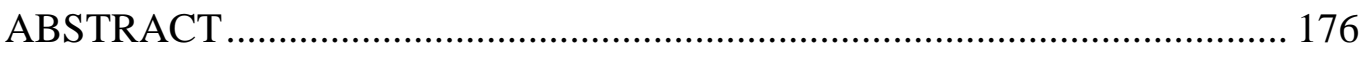

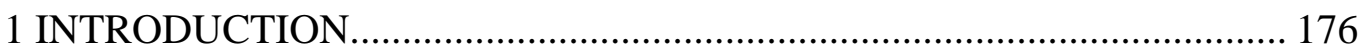

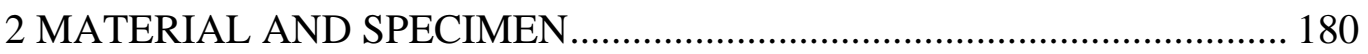

2.1 SKIN (FACESHEET) AND CORE MATERIALS ............................. 180

2.2 CORE-SHELL RUBBER (CSR) TOUGHENED SANDWICH

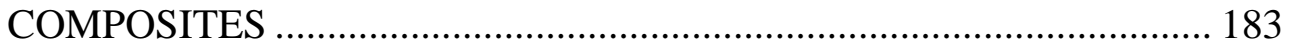

3 EXPERIMENTAL SET-UP AND PROCEDURE ……………………….... 184

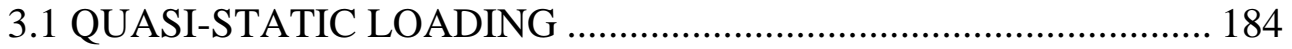

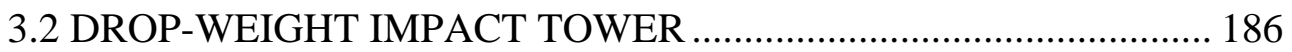

3.3 SPLIT HOPKINSON PRESSURE BAR (SHPB) .............................. 190

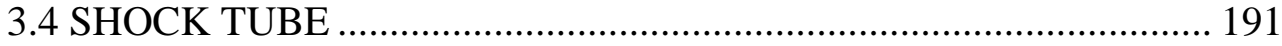

3.5 HIGH-SPEED PHOTOGRAPHY SYSTEM ..................................... 194

3.6 EXPERIMENTAL PROCEDURE AND PARAMETERS.................. 196

3.7 RESIUDAL COMPRESSIVE STRENGTH MEASUREMENTS ..... 198

4 EXPERIMENTAL RESULTS AND DISCUSSION ..................................... 199

4.1 CONSTITUTIVE BEHAVIOR OF FACESHEET AND CORE

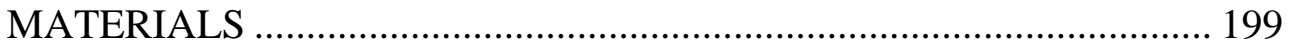

4.1.1 QUASI-STATIC BEHAVIOR ................................................. 199 
4.1.3 HIGH STRAIN RATE (SHPB) BEHAVIOR .......................... 202

4.2 BLAST RESPONSE OF CORE-SHELL RUBBER (CSR)

TOUGHENED SANDWICH COMPOSITES .......................................... 203

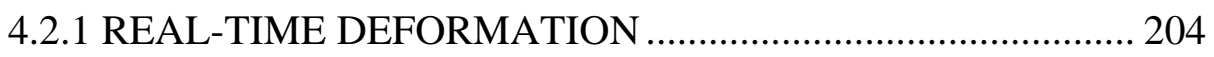

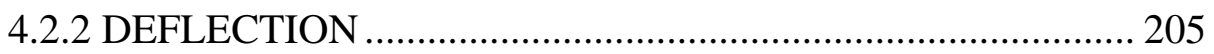

4.2.3 DIGITAL IMAGE CORRELATION (DIC) ANALYSIS ........ 207

4.2.4 RESIDUAL COMPRESSIVE STRENGTH ........................... 214

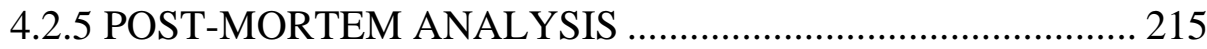

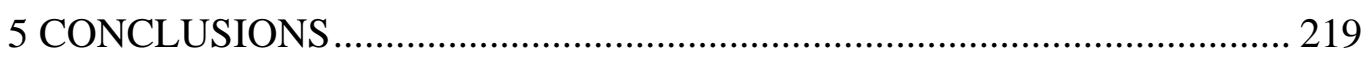

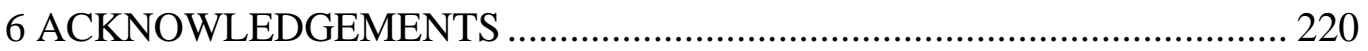

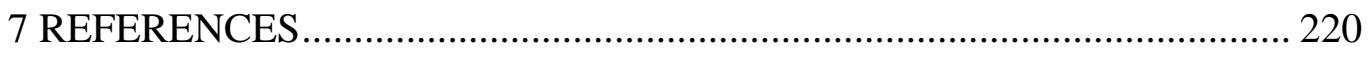

CHAPTER 7: CONCLUSIONS AND FUTURE WORK ....................................... 225

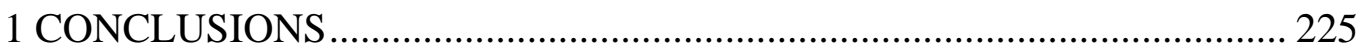

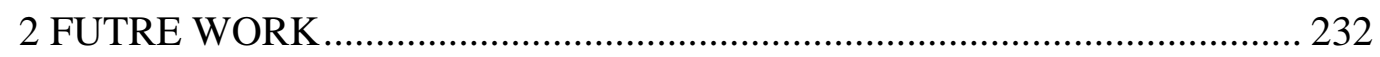

APPENDICES …..................................................................................................................235

APPENDIX A: FABRICATION OF SAMPLES (VARTM PROCESS) ........ 235

APPENDIX B: STANDARD OPERATING PROCEDURES (SOP),............. 243

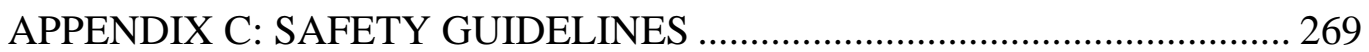

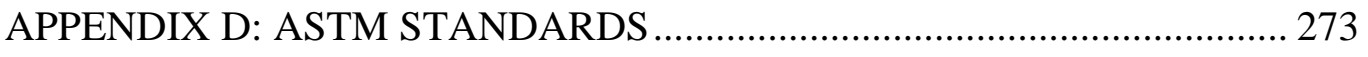

BIBLIOGRAPHY …........................................................................................................... 278 


\section{LIST OF TABLES}

TABLE

PAGE

\section{CHAPTER 2}

Table 1 - Material properties for foam core

Table 2 -Yield strengths of Corecell ${ }^{\mathrm{TM}}$ A-series foams .27

\section{CHAPTER 3}

Table 1 - Quasi-static material properties of the foam core and EVE facesheets ........50

Table 2 - Foam core gradation and thickness ..............................................52

Table 3 - Flow stresses (plateau) of Corecell ${ }^{\mathrm{TM}}$ A-series foams ...............................58

Table 4 - Dynamic strain required to initiate compression in subsequent foam core

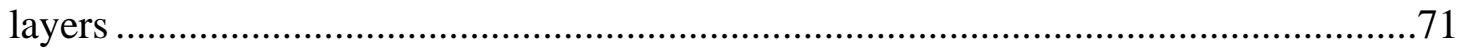

Table 5 - Permanent deflection and core compression..........................................85

\section{CHAPTER 4}

Table 1 -Material properties of the foam core and polyurea ...................................99

Table 2 - Yield strength of core materials .........................................................106

\section{CHAPTER 5}

Table 1 - Quasi-static material properties of the foam core and EVE facesheets ......135

Table 2 - Flow stresses (plateau) of Corecell ${ }^{\mathrm{TM}}$ A-series foams .............................147

Table 3 - Specimen dimensions and three-point bending variables ........................165

Table 4 - Comparison of quasi-static flexural and shear rigidity of both configurations 166 
Table 5 - Comparison of different three-point bending failure modes and required

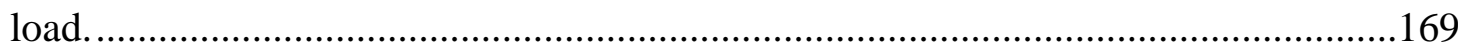

Table 6 - Overall comparison of final results .....................................................171

\section{CHAPTER 6}

Table 1 - Quasi-static material properties of the foam core and EVE facesheets ......182

Table 2 - Mass of drop-weight components contributing to impact 188 


\section{LIST OF FIGURES}

FIGURE

PAGE

\section{CHAPTER 2}

Figure 1 - Sketch of the incident and the reflected shock process .17

Figure 2 - Deflection based on high-speed images and pressure profile. 20

Figure 3 - Specimen configurations .23

Figure 4 - Sketch of modified SHPB with hollow transmitter bar and typical pulse profiles .24

Figure 5 - Shock tube apparatus .25

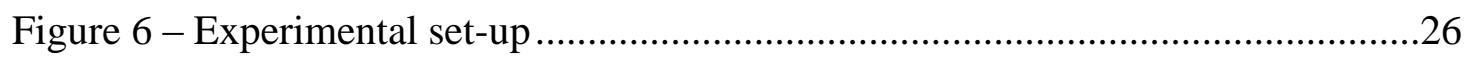

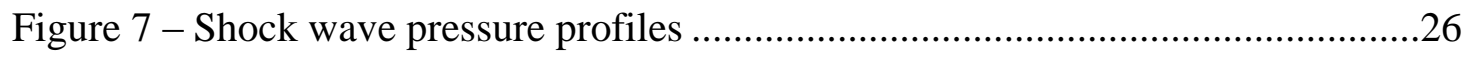

Figure 8 - Quasi-static and high strain rate behaviors of different types of Corecell ${ }^{\mathrm{TM}}$ A-series foams .27

Figure 9 - Real-time side-view images of Configuration 1 (A300/A500/A800) .........29

Figure 10 - Real-time side-view images of Configuration 2 (A500/A300/A800) ........31

Figure 11 - Deflection of A300/A500/A800 ......................................................33

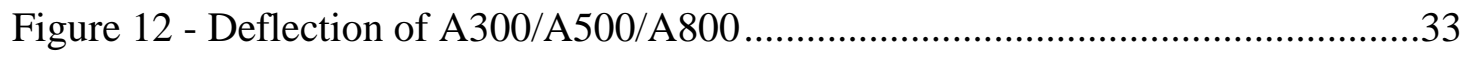

Figure 13 - Visual examination of sandwich composites after being subjected to highintensity blast load .34

Figure 14 - The failure and compression of core foam cell microstructure in Configuration 1(A300/A500/A800). .35 
Figure 15 - The details of the failure mode in Configuration 2 (A500/A300/A800) ...36

Figure 16 - Incident and reflected energies of Configuration 1 (A300/A500/A800)...37

Figure 17 - A comparison of the total energy loss and a comparison of the deformation energy for the two configurations .38

\section{CHAPTER 3}

Figure 1 - Schematic of sandwich composite with skin and core .49

Figure 2 - Cell microstructure of foam core layers …………………......................50

Figure 3 - Specimen configuration and core gradation ...........................................51

Figure 4 - Sketch of modified SHPB device with hollow transmission bar and typical

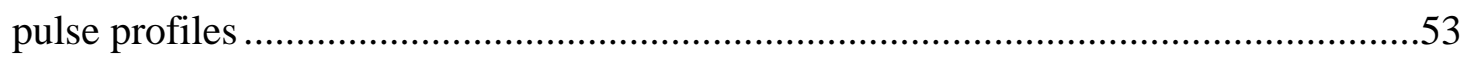

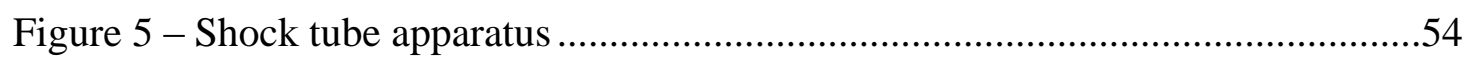

Figure 6 - High-speed photography set-up (Back-view DIC and side-view)................55

Figure 7 - Incident and reflected pressure profiles ...................................................56

Figure 8 - Compressive behavior of different types of Corecell ${ }^{\mathrm{TM}}$ A-series foams .....57

Figure 9 - High-speed images for (a) One layer (b) Two layers (c) Three layers and

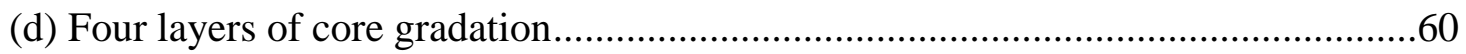

Figure 10 - Mid-point deflections of all core configurations .......................................65

Figure 11 - Strain history of each core layer along the mid-line (line of symmetry) for

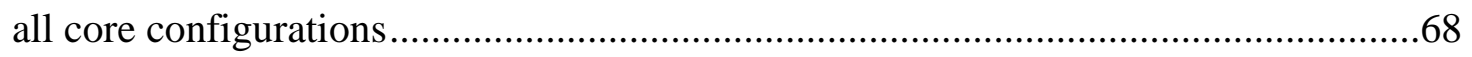

Figure 12 - Front and back face deflection comparison of all core configurations ......72

Figure 13 - Localized areas of larger deflections (loading) during fluid-structure 
interaction. 74-75

Figure 14 - Full-field out-of-plane deflection (W) for (a) One layer (b) Two layers (c) Three layers and (d) Four layers of core gradation .76

Figure 15 - Full-field in-plane strain $\left(\varepsilon_{y y}\right)$ for (a) One layer (b) Two layers (c) Three layers and (d) Four layers of core gradation ...... .77

Figure 16 - In-plane strain $\left(\varepsilon_{\mathrm{yy}}\right)$ of all core configurations .79

Figure 17 - Out-of-plane velocity (dW/dt) of all core configurations .80

Figure 18 - Visual examination of all core configurations after being subjected to highintensity blast loading .81

Figure 19 - Specimen schematic for permanent deflection measurements

\section{CHAPTER 4}

Figure 1 - Schematic of sandwich composite with skin and core .98

Figure 2 - Cell microstructure of foam core layers ...

Figure 3 - Specimen configuration and core gradation .100

Figure 4 - Sketch of modified SHPB device with hollow transmission bar and typical pulse profiles. .101

Figure 5 - Shock tube apparatus

Figure 6 - High-speed photography set-up (back-view DIC and side-view) .103

Figure 7 - Incident and reflected pressure profiles. .104

Figure 8 - Quasi-static and high strain-rate behaviors of the different types of core materials .105 
Figure 9 - High-speed images for configuration 1 (PU/A300/A500/A800) ..............106

Figure 10 - High-speed images for configuration 2 (A300/A500/A800/PU) 107

Figure 11 - Mid-point deflection of both configurations

Figure 12 - Strain and strain rate history of each core layer along the mid-line (line of symmetry) for both configurations

Figure 13 - Front and back face out-of-plane velocities for both configurations 115

Figure 14 - Full-field out-of-plane deflection (W) of both configurations 116

Figure 15 - Full-field in-plane-strain $\left(\varepsilon_{\mathrm{yy}}\right)$ of both configurations

Figure 16 - Full-field out-of-plane velocity (dW/dt) of both configurations 118

Figure 17 - In-plane strain $\left(\varepsilon_{\mathrm{yy}}\right)$ of both configurations

Figure 18 - Visual examination of both configurations after being subjected to high intensity blast load (Incident peak pressure $\sim 1.0 \mathrm{MPa}$ ) 120

Figure 19 - Visual examination of both configurations after being subjected to high intensity blast load (Incident peak pressure $~ 1.5 \mathrm{MPa}$ ) .121

Figure 20 - Total energy loss in both configurations during blast loading event .......123

Figure 21 - Deformation energy of both configurations during blast loading event ..124

\section{CHAPTER 5}

Figure 1 - Schematic of sandwich composite with skin and core. .135

Figure 2 - Cell microstructure of foam core layers .136

Figure 3 - Specimen design and core configuration. 138

Figure 4 - Sketch of modified SHPB device with hollow transmission bar and typical 
pulse profiles

Figure 5 - Shock tube apparatus

Figure 6 - High-speed photography set-up (Back-view DIC and side-view)

Figure 7 - Incident and reflected pressure profile 145

Figure 8 - Compressive behavior of both types of Corecell $^{\mathrm{TM}}$ A-series foams 146

Figure 9 - High-speed images for sandwich composites with (a) Equivalent core layer thickness (b) Equivalent core layer mass .148

Figure 10 - Mid-point deflections of both core configurations

Figure 11 - Comparison of mid-point deflections of the back face of both configurations.

Figure 12 - Strain of A300 foam core layer along the mid-line (line of symmetry) for both configurations

Figure 13 - Full-field out-of-plane deflection (W) of both configurations 154

Figure 14 - Full-field in-plane strain $\left(\varepsilon_{y y}\right)$ of both configurations .155

Figure 15 - Full-field out-of-plane velocity (dW/dt) of both configurations 156

Figure 16 - In-plane strain $\left(\varepsilon_{\mathrm{yy}}\right)$ of both core configurations 158

Figure 17 - Out-of-plane velocity (dW/dt) of both core configurations 158

Figure 18 - Visual examination of all core configurations after being subjected to highintensity blast loading 160

Figure 19 - Energy redistribution behavior of both configurations during the blast loading event .161 
Figure 20 - Shape reconstruction analysis of both configurations 163

Figure 21 - Simply-supported sandwich beam centrally loaded in three-point bending. 164

Figure 22 - Modified simply-supported sandwich beam centrally loaded in three-point bending for both core configurations

\section{CHAPTER 6}

Figure 1 - Schematic of sandwich composite with skin and core. 181

Figure 2 - SEM image of nano-scale core-shell rubber (CSR) particles.

Figure 3 - Cell microstructure of A500 foam core layer.

Figure 4 - Specimen design and facesheet configurations

Figure 5 - Quasi-static experimental set-up 186

Figure 6 - Drop-weight impact experimental set-up 187

Figure 7 - Specimen placed in simply-supported conditions for drop-weight impact event

Figure 8 - Sketch of modified SHPB device with hollow transmission bar and typical pulse profiles

Figure 9 - Shock tube apparatus

Figure 10 - High-speed photography set-up (Back-view DIC and side-view) 196

Figure 11 - Incident and reflected pressure profile

Figure 12 - Compressive residual strength experimental set-up. .198

Figure 13 - Quasi-static behavior of composite facesheets (tensile) and A500 foam 
core (compressive)

Figure 14 - Total energy absorbed for both composite facesheets during drop-weight impact event

Figure 15 - High strain rate compressive behavior of composite facesheets and A500 foam core. .203

Figure 16 - High-speed images for (a) Non-CSR and (b) CSR toughened sandwich composites .204

Figure 17 - Mid-point deflections of both configurations. .206

Figure 18 - Localized areas of larger deflections (loading) during fluid-structure interaction .208

Figure 19 - Full-field out-of-plane deflection (W) of both configurations .209

Figure 20 - Full-field in-plane strain $\left(\varepsilon_{y y}\right)$ of both configurations .210

Figure 21 - Full-field out-of-plane velocity (dW/dt) of both configurations .211

Figure 22 - In-plane strain $\left(\varepsilon_{\mathrm{yy}}\right)$ of both configurations .213

Figure 23 - Out-of-plane velocity (dW/dt) of both configurations

Figure 24 - Longitudinal line-inspection analysis across central region of back face for both composite facesheet systems.

Figure 25 - Residual compressive strength of both types of composite facesheets after being subjected to high intensity blast loading (incident peak pressure $0.3 \mathrm{MPa}$ ) ....215 Figure 26 - Visual examination of both composite facesheets after being subjected to $150 \mathrm{~J}$ impact .216 
Figure 27 - Visual examination of both composite facesheets after being subjected to

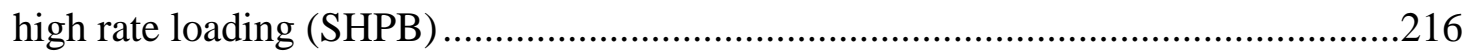
Figure 28 - Visual examination of both configurations after being subjected to high intensity blast load (incident peak pressure of 1.0 MPa)

Figure 29 - Visual examination of both composite facesheets after being subjected to high intensity blast loading (incident peak pressure $0.3 \mathrm{MPa}$ ) .218 


\section{CHAPTER 1}

\section{INTRODUCTION AND LITERATURE REVIEW}

Blast loading and explosions in the form of terrorist attacks are occurring much more frequently in the world we live in today, i.e. attack on USS Cole [1] (Naval structure), attack on the US Embassy in Kenya [2] (Civilian structure) and IED roadside bombings on Humvees (Military structures). These attacks have led to a significant need to replace conventional structural materials with more blast resistant materials. Also these attacks have lead to a need to develop a more comprehensive understanding of material and damage behavior under air-blast and explosive loadings. With growing concerns on safety and human lives involved, the significance of such research cannot be understated.

Sandwich structures have very important applications in the naval and aerospace industry. Due to their construction they have many advantages that include high strength/weight ratio, high stiffness/weight ratio, and energy absorption capabilities. Sandwich structures consist of two thin, stiff facesheets, usually the same thickness, separated by a lightweight, thicker core. The facesheets carry almost all of the bending and in-plane loads, while the core helps to stabilize the facesheets and defines the flexural stiffness and out-of-plane shear and compressive behavior. When sandwich structures are subjected to high-intensity impulse loadings, such as air blasts, the core materials play a crucial role in the dynamic behavior and overall structural response. Their properties assist in dispersing the mechanical impulse that is transmitted into the structure, and thus protect anything located behind it [3-5]. 
Common cores are made of metallic and non-metallic honeycombs, cellular foams, balsa wood, PVC, truss and lattice structures. Extensive research exists in the literature regarding the dynamic response of sandwich structures consisting of the various core materials and geometric structures. Dharmasena et al. [5], Zhu et al. [6], and Nurick et al. [7] have tested sandwich structures with a metallic honeycomb core material. Their results indicated that the parameters of core materials can effectively reduce the transmitted impulse and damage of the back facesheet. Tagarielli et al. [8] has investigated the dynamic response of sandwich beams with PVC and balsa wood cores. Radford et al. [9] has conducted metal foam projectile impact experiments to simulate a blast loading on sandwich structures with metal foam cores. McShane et al. $[10,11]$ have investigated the underwater blast response of sandwich composites with a prismatic lattice (Y-frame, corrugated), as well as simulated an air blast, using metal foam projectiles, on sandwich composites with a pyramidal lattice cores. These studies have indicated that advanced sandwich structures can potentially have significant advantages over monolithic plates of equivalent mass in absorbing the blast energy, whether in air or underwater.

In recent years, functionally graded materials, where the material properties vary gradually or layer by layer within the material itself, have gained much attention. Hossain et al. [12] have experimentally studied the compressive behavior of a functionally graded foam material system and energy absorption under quasi-static loading conditions. The results indicated stepwise crushing from the lower density to the higher density foams. Kiernan et al. [13] numerically investigated the propagation of a stress wave through a virtual functionally graded foam material (FGFM). It was 
concluded that the amplitude of a stress wave propagating through a FGFM can be shaped by the gradient functions according to which the foam density varies through the direction of wave propagation. Cui et al. [14] proposed a functionally graded foam model to improve the energy absorption characteristics offered by uniform foams. In this model, the characteristics of the foam (e.g. density) are varied through the thickness. Results indicated that for high energy impacts, increasing the density range can decrease the performance of the graded foams over conventional foams of uniform density. Consequently, decreasing the density range can increase the performance of the graded foams over conventional foams of uniform density.

Since the properties of the layered/graded material can be designed and controlled, they show great potential to be an effective core material for energy absorption and blast mitigation. To date, there have been very little results published regarding the dynamic impact response of sandwich composites with a functionally graded core, and even less regarding the blast response. Li et al. [15] numerically examined the response of layered and graded metal-ceramic structures under impulsive loadings. It was concluded that the choice of gradation has a great significance on the impact applications and the particular design can exhibit better energy dissipation properties. Apetre et al. [16] numerically investigated the impact response of sandwich beams with a functionally graded core. Their results showed that a reasonable core design can effectively reduce the shear forces and strains within the structure. Consequently, they can mitigate or completely prevent impact damage on sandwich composites. However, there have been no results past or present, regarding the properties of sandwich composites with a functionally graded core material under 
blast loading. To obtain a better understanding and optimal core design of sandwich composites to improve blast performance, the dynamic constitutive behavior of the core material has to be experimentally studied first. Also, the dynamic deformation and failure mechanisms under blast loading have to be investigated as well.

In recent years, with its ability to improve structural performance and damage resistance of structures, the application of polyurea to sandwich structures has become a new area of interest. Although the behavior of polyurea has been investigated [1720], there have been no studies regarding the dynamic behavior of functionally graded core with a polyurea interlayer. Tekalur et al. [21] experimentally studied the blast resistance and response of polyurea based layered composite materials subjected to blast loading. Results indicated that sandwich materials prepared by sandwiching the polyurea between two composite skins had the best blast resistance compared to the EVE composite and polyurea layered plates. Bahei-El-Din et al. [22] numerically investigated the blast resistance of sandwich plates with a polyurea interlayer under blast loading. Their results suggest that separating the composite facesheet from the foam core by a thin interlayer of polyurea can be very beneficial in comparison to the conventional sandwich plate design. Amini et al. [23 - 26] experimentally and numerically studied the dynamic response of circular monolithic steel and steelpolyurea bilayer plates to impulsive loads. More importantly they focused on the significance of the relative position of the polyurea layer with respect to the loading direction. Results indicated that the polyurea layer can have a significant effect on the response of the steel plate to dynamic impulsive loads, both in terms of failure mitigation and energy absorption, if it is placed on the back face of the plate. On the 
contrary, they also found that polyurea can enhance the destructive effect of the blast, promoting (rather than mitigating) the failure of the steel plate if applied on the impact side of the plate.

Apart from the various core materials, the facesheet also plays an important role in the blast mitigation properties of the structure. In fact, the facesheet is the part of the structure which is directly exposed to the blast loading. Adhesive and fibercomposite materials are commonly based on epoxy polymers. The epoxies are highly cross-linked thermosetting polymers, which exhibit good elevated temperature resistance and low creep. However their high cross-link densities cause them to be relatively brittle in nature. This limits their applications as structural materials, as they have a poor resistance to crack initiation and growth.

To overcome this deficiency, and increase toughness, a commonly used method is the addition of a second dispersed particulate phase (during infusion). This second dispersed particulate phase can either be initially soluble in the epoxy resin and which then phase separates during curing to form or it can be of pre-formed particles. For the phase-separable tougheners, both rubbers (carboxyl-terminated butadiene-acrylonitrile (CBTN) [27, 28]) and thermoplastics [29-31] have been investigated. Pre-formed particles that have been studied include ceramic particles (glass [32, 33], alumina [34], or silica [35,36]), metal particles (aluminum [35]), polymers [37, 38] and core-shell rubber particles [39-45].

The behavior of rubber toughened and core-shell rubber toughened epoxy resin has been extensively studied in the literature [27, 28, 39-45]. The core-shell rubber particles consist of two parts, a core which is rubber for impact resistance, and a shell 
which is a co-polymer compatible with epoxy resin. Note for these investigations, most of these rubber particles were on the micro-scale level. Results of these investigations indicated that the addition of rubber particles to epoxy resins can aid in increasing the fracture toughness, lap shear / T-peel strength, and fatigue resistance, as well as allow for no loss of $\mathrm{T}_{\mathrm{g}}$ or thermal properties (during infusion process), consistent morphology and a wide cure window. Therefore, the addition of rubber particles to current resin systems allows the once-brittle by nature resin to become toughened and subsequently more impact resistant.

Due to the improvement in mechanical properties, these rubber toughened epoxies can be used as the matrices for fiber-reinforced composite systems. However, the addition of these tougheners or pre-formed rubber particles, in the concentrations required to sufficiently enhance the toughness, can significantly increase the viscosity of the matrix resin. Also, conventional pre-formed particles generally have a particle diameter larger than the inter-fiber spacing, and particles are filtered out during infusion. This has led to the development of nano-scale rubber particles [44], defined as rubber particles less than $100 \mathrm{~nm}$ in diameter, since these particles will flow between the fibers during infusion [45]. However, research investigating nano-scale rubber toughened fiber-reinforced composite systems is extremely limited. 


\section{REFERENCES}

[1] Perl R and O'Rourke R (2001) Terrorist attack on USS Cole: Background and issues for Congress. Emerging Technologies: Recommendations for counter-terrorism. Institute for Security Technology Studies, Dartmouth College.

[2] Perl R (1998) Terrorism: US responses to bombings in Kenya and Tanzania: A new policy direction? Congressional Report. Congressional Research Service, The Library of Congress.

[3] Xue Z and Hutchinson JW (2003) Preliminary assessment of sandwich plates subject to blast loads. Int J Mech Sci, 45: 687 - 705.

[4] Fleck NA and Deshpande VS (2004) The resistance of clamped sandwich beams to shock loading. J Appl Mech, 71: 386 - 401.

[5] Dharmasena KP, Wadley HNG, Xue Z and Hutchinson JW (2008) Mechanical response of metallic honeycomb sandwich panel structures to high-intensity dynamic loading. Int J Imp Eng, 35 (9): 1063 - 1074.

[6] Zhu F, Zhao L, Lu G and Wang Z (2008) Deformation and failure of blast loaded metallic sandwich panels - Experimental investigations. Int J Imp Eng, 35 (8): 937 951.

[7] Nurick GN, Langdon GS, Chi Y and Jacob N (2009) Behavior of sandwich panels subjected to intense air blast: part 1- Experiments. Compos Struct, 91 (4): 433 - 441.

[8] Tagarielli VL, Deshpande VS and Fleck NA (2008) The high strain rate response of PVC foams and end-grain balsa wood. Compos: Part B, 39: 83 - 91.

[9] Radford DD, McShane GJ, Deshpande VS and Fleck NA (2006) The response of clamped sandwich plates with metallic foam cores to simulated blast loading. Int $\mathbf{J}$ Solid Struct, 44: 6101 - 6123.

[10] McShane GJ, Deshpande VS and Fleck NA (2007) The underwater blast resistance of metallic sandwich beams with prismatic lattice cores. J Appl Mech, 74: $352-364$. 
[11] McShane GJ, Radford DD, Deshpande VS and Fleck NA (2006) The response of clamped sandwich plates with lattice cores subjected to shock loading. European J Mech - A: Solids, 25: 215 - 229.

[12] Hossain MK, Liu QL and O'Toole BJ (2007) Functionally graded foam material system for energy absorption. SAMPE $39^{\text {th }}$ ISTC- Cincinnati OH, Oct. 29 - Nov. 1.

[13] Kiernan S, Cui L and Gilchrist MD (2009) Propagation of a stress wave through a virtual functionally graded foam. Int J Non-Linear Mech, 44: 456 - 468.

[14] Cui L, Kiernan S and Gilchrist MD (2009) Designing the energy absorption capacity of functionally graded foam materials. Mater Sci Eng: A, 507: 215 - 225.

[15] Li Y, Ramesh KT and Chin ESC (2001) Dynamic characterization of layered and graded structures under impulsive loading. Int J Solid Struct, 38 (34-35): 6045 - 6061.

[16] Apetre NA, Sankar BV and Ambur DR (2006) Low-velocity impact response of sandwich beams with functionally graded core. Int J Solid Struct, 43(9): 2479 - 2496.

[17] Yi J, Boyce MC, Lee GF and Balizer E (2005) Large deformation rate-dependent stress-strain behavior of polyurea and polyurethanes. Poly, 47 (1): 319 - 329.

[18] Amirkhizi AV, Isaacs J, McGee J and Nemat-Nasser S (2006) An experimentally-based constitutive model for polyurea, including pressure and temperature effects. Phil Mag, 86 (36): 5847 - 5866.

[19] Hoo Fatt MS, Ouyang X and Dinan RJ (2004) Blast response of walls retrofitted with elastomer coatings. Struct Mater, 15: 129 - 138.

[20] Roland CM, Twigg JN, Vu Y and Mott PH (2006) High strain rate mechanical behavior of polyurea. Poly, 48 (2): 574 - 578.

[21] Tekalur SA, Shukla A and Shivakumar K (2008) Blast resistance of polyurea based layered composite materials. Compos Struct, 84: 271 - 281.

[22] Bahei-El-Din YA, Dvorak GJ and Fredricksen OJ (2006) A blast-tolerant sandwich plate design with a polyurea interlayer. Int J Solid Struct, 43: 7644 - 7658. 
[23] Amini MR, Isaacs JB and Nemat-Nasser S (2010) Experimental investigation of response of monolithic and bilayer plates to impulsive loading. Int J Imp Eng, 37: 82 89.

[24] Amini MR, Amirkhizi AV and Nemat-Nasser S (2010) Numerical modeling of response of monolithic and bilayer plates to impulsive loading. Int J Imp Eng, 37: 90 102.

[25] Amini MR, Isaacs JB and Nemat-Nasser S (2010) Numerical modeling of effect of polyurea on response of steel plates to impulsive loads in direct pressure-pulse experiments. Mech Mater, 42: 615 - 627.

[26] Amini MR, Isaacs JB and Nemat-Nasser S (2010) Investigation of effect of polyurea on response of steel plates to impulsive loads in direct pressure-pulse experiments. Mech Mater, 42: 628-639.

[27] Kinloch AJ, Shaw SJ, Tod DA, Hunston DL (1983) Deformation and fracture behavior of a rubber-toughened epoxy: 1. Microstructure and fracture studies. Poly, 24 (10): 1341- 1354.

[28] Yee AF, Pearson RA (1986) Toughening mechanisms in elastomer-modified epoxies. J Mater Sci, 21: 2462-2474.

[29] Bucknall CB and Partridge IK (1983) Phase separation in epoxy resins containing polyethersulphone. Poly, 24 (5): $639-644$.

[30] Kinloch AJ, Yuen ML, Jenkins SD (1994) Thermoplastic-toughened epoxy polymers. J Mater Sci, 29: 3781-3790.

[31] Johnsen BB, Kinloch AJ, Taylor AC (2005) Toughness of syndiotactic polystyrene/epoxy polymer blends: microstructure and toughening mechanisms. Poly, 46: 7352-7369.

[32] Broutman LJ, Sahu S (1971) The effect of interfacial bonding on the toughness of glass filled polymers. Mater Sci Eng, 8: 98-107.

[33] Spanoudakis J, Young RJ (1984) Crack propagation in a glass particle- filled epoxy resin - Part 1. J Mater Sci, 19: 473-486. 
[34] McGrath LM, Parnas RS, Lenhart JL, King S (2006) Fracture toughness of alumina-epoxy composites. Poly Mater Sci Eng, Preprints, 94: 683 - 684.

[35] Griffiths R, Holloway D (1970) The fracture energy of some epoxy resin materials. J Mater Sci, 5 (4): 302-307.

[36] Hsieh TH, Kinloch AJ, Masania K, Sohn Lee J, Taylor AC, Sprenger S (2010) The toughness of epoxy polymers and fiber composites modified with rubber microparticles and silica nanoparticles. J Mater Sci, 45: 1193 - 1210.

[37] Dixon DG, Harris SJ, Dempster M, Nicholls P (1998) Effect of PEEK fibers and powder on joints made with high temperature adhesive. J Adhesion, 65 (1- 4): 131162.

[38] Kinloch AJ, Taylor AC (2002) The toughening of cryanate-ester polymers -Part I - Physical modification using particles, fibers and woven mats. J Mater Sci, 37: 433460.

[39] Lin KF, Shieh YD (1998) Core-Shell Particles Designed for Toughening the Epoxy Resins: I. Preparation and Characterization of Core-Shell Particles. J Appl Poly Sci, 69: 2069-2078.

[40] Lin KF, Shieh YD (1998) Core-shell particles designed for toughening the epoxy resins: II. Core-shell particle toughened epoxy resins. J Appl Poly Sci, 70: 2313 -2322. [41] Becu L, Maazouz A, Sautereau H, and Gerard JF (1997) Fracture behavior of epoxy polymers modified with core-shell rubber particles. Journal J Appl Poly Sci, 65 (12): 2419-2431.

[42] Yan C, Xiao KQ, Ye L, and Mai YW (2002) Numerical and experimental studies on the fracture behavior of rubber toughened epoxy in bulk specimen and laminated composites. J Mater Sci, 37 (5): 921-927.

[43] Giannakopoulos G ,Masania K, and Taylor AC (2011) Toughening of epoxy using core-shell particles. J Mater Sci, 46 (2): 327-338.

[44] Kaneka Texas Corporation, http://www.kanekatexas.com/MX (Accessed April 3, 2012). 
[45] Kinloch AJ, Mohammed RD, Taylor AC, Sprenger S and Egan D (2006) The interlaminar toughness of carbon-fiber reinforced plastic composites using 'hybridtoughened' matrices. J Mater Sci, 41(15): 5043-5046. 


\title{
CHAPTER 2
}

\section{THE BLAST RESISTANCE OF SANDWICH COMPOSITES WITH STEPWISE GRADED CORES}

by

\author{
Nate Gardner, Erheng Wang and Arun Shukla
}

has been published in the International Journal of Solids and Structures (2009, Vol. 46, 3492-3502)

Corresponding Author: Arun Shukla

Dynamic Photo Mechanics Laboratory

Department of Mechanical, Industrial and Systems

Engineering

University of Rhode Island

206 Wales Hall, 92 Upper College Rd

Kingston, RI, 02881, USA

Phone: +1-401-874-2283

Email Address: shuklaa@egr.uri.edu 


\begin{abstract}
Shock tube experiments were performed to study the dynamic response of sandwich panels with E-glass Vinyl-Ester (EVE) composite facesheets and stepwise graded styrene acrylonitrile (SAN) foam cores. Two types of core configurations, with identical areal density, were subjected to the shock wave loading. The core layers were arranged according to the density of the respective foam; configuration 1 consisted of low / middle / high density foams and configuration 2 consisted of middle / low / high density foams. The method to calculate the incident and reflected energies of the shock wave, as well as the deformation energy of the specimen, were proposed based on the shock wave pressure profiles and the high-speed deflection images that were obtained. The experimental results showed that configuration 1 outperformed configuration 2 in regards to their blast resistance. Significant core material compression was observed in configuration 1 , while in configuration 2 the core layers disintegrated and the front skin (blast side) fractured into two pieces along the midsection. The estimated energies were then calculated for both configurations. The total energy difference between the incident and reflected energies was almost identical, even though the deformation energy for configuration 2 was larger.
\end{abstract}

Keyword: Sandwich Structures, Discretely Layered Core, Shock Wave Loading, Dynamic Failure, High-speed Imaging

\title{
1. Introduction
}

Sandwich structures have important applications in the naval and aerospace industry. Their high strength/weight ratio and high stiffness/weight ratio play a vital role in their applications, especially when they are subjected to high-intensity impulse 
loadings such as air blasts. Their properties assist in dispersing the mechanical impulse that is transmitted into the structure and thus protect anything located behind it (Xue et al. 2003; Fleck and Deshpande, 2004; Dharmasena et al., 2008).

The core materials play a crucial role in the dynamic behavior of sandwich structures when they are subjected to blast loading. The general core materials include polymer foams, metal foams, metal honeycomb, balsa wood, and truss structures et al. In recent years, stepwise graded materials, where the material properties vary gradually or layer by layer within the material itself, were utilized as a core material in sandwich composites. Since the properties of graded/layered core structures can be designed and controlled, they show great potential to be an effective core material for absorbing the blast energy and improving the overall blast resistance of sandwich structures.

The behaviors of sandwich composites under blast loading have been widely studied. Fleck and Deshpande (2004) have theoretically studied the dynamic response of sandwich beams under air and underwater blast loading. They divided the structural response into three sequential steps and then developed performance charts of the sandwich beams with different core materials in order to find an optimal design. Dharmasena et al. (2008), Nurick et al. (2008), and Zhu et al. (2008) have tested sandwich structures with a metallic honeycomb core material. Their results indicated that the parameters of core materials can effectively reduce the damage of the back facesheet. Radford et al. (2006) has conducted metal foam projectile impact experiments to simulate a blast loading on sandwich structures with metal foam cores, and he found that the ability of sandwich panels to resist dynamic loading is far more 
superior to that of monolithic metal plates with the same areal density. Tekalur et al (2008) have studied the dynamic behavior of sandwich structures with reinforced polymer foam cores. They concluded that the imparted damage was substantially reduced when Z-direction pin reinforcements were introduced into the core material. Li et al. (2008) proposed a higher order non-linear core theory and incorporated it into the constitutive equations of core materials. They used this model to obtain the transient response of a shallow shell sandwich composite subjected to blast loading. In addition to the previous works, the behaviors of sandwich structures with designable micro structure core materials have been studied under blast loading in recent years (McShane et al. 2006; Wadley et al. 2008).

However, there have been no results, past or present, regarding the dynamic properties of sandwich composites with a stepwise graded core material under blast loading. Only the behaviors of sandwich composites with stepwise graded core under contact loadings, such as low-velocity impact, have been reported, and even these reports are limited. The numerical investigation by Apetre et al. (2006) has shown that a reasonable core design can effectively reduce the shear forces and strains within the structures. Consequently, they can mitigate or completely prevent impact damage on sandwich composites. Li et al. (2001) examined the impact response of layered and graded metal-ceramic structures numerically. He found that the choice of gradation has a great significance on the impact applications and the particular design can exhibit better energy dissipation properties.

The present study focuses on the blast resistance and energy absorption of sandwich composites with a stepwise graded foam core when experimentally 
subjected to a shock wave loading. The results will help to understand the performance and the mechanisms of failure of sandwich composites with a stepwise graded core under blast loading and provide a guideline for a better core design. The quasi-static and dynamic constitutive behaviors of the foam core materials were first studied using a modified SHPB device with a hollow transmitter bar. The sandwich composites with two types of layered foam core arrangements were then fabricated and subjected to shock wave loading generated by a shock tube. The two types of sandwich composites have identical core materials but different core layer arrangements. The shock pressure profiles and real-time deflection images were carefully analyzed to reveal the failure mechanisms of these sandwich composites.

Based on the experimental data, the methods to calculate the energies of the incident shock wave (incident energy), the reflected shock wave (reflected energy) and the energy that deforms the specimen (deformation energy) were proposed and implemented. The energy redistribution in the system was analyzed, and the results showed that only a small amount of incident energy of the shock wave was transferred into the sandwich composites during the shock wave loading process.

\section{Energy Evaluation}

The incident energy, the reflected energy, and the deformation energy were calculated based on the shock wave pressure profiles and the high-speed deflection images obtained from the shock tube experiment. Fig. 1 shows a shock wave loading process with a shock tube. The definite state of the gas can be defined using the following physical parameters: 
$p \quad$ the pressure

$\rho \quad$ the gas density

$u \quad$ the gas particle speed

$c \quad$ the speed of sound in gas

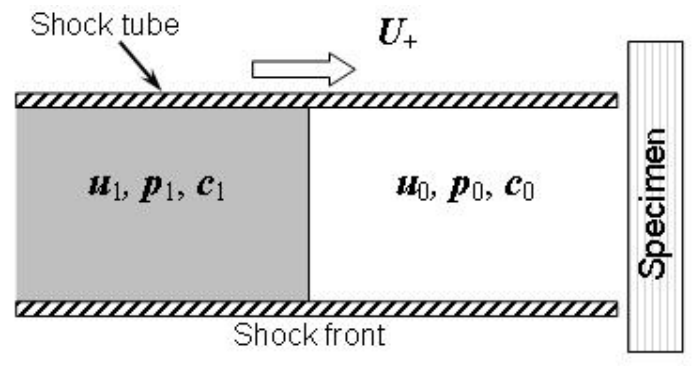

(a) Incident shock process

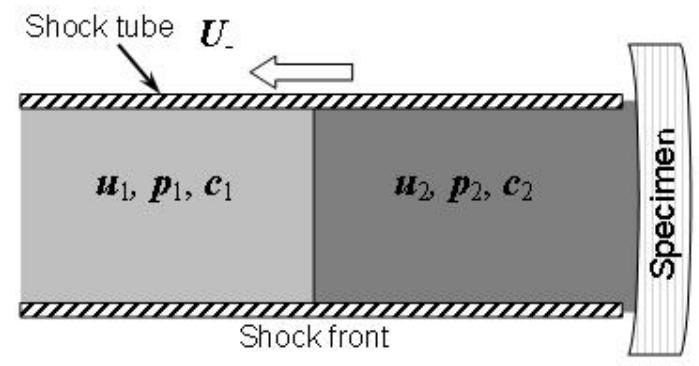

(b) Reflected shock process

Fig. 1 Sketch of the incident and the reflected shock process

The subscript 0 on the parameters denotes the initial state of the gas. Subscript 1 represents the state of the gas located behind the incident shock wave front and it will be defined as the incident state. Subscript 2 represents the state of the gas located behind the reflected shock wave front and it will be defined as the reflected state.

\subsection{The Incident and Reflected Energies}

The calculation of the incident and reflected energies is based on the incident and reflected shock wave pressure profiles. When a planar shock wave impacts a planar panel, the energy stored in the gas, which is located behind the shock wave front, will impinge on the structure. The stored energy in the gas is equivalent to the work done by the gas as it propagates through the cross-section of the shock tube. Note that the particle speed, $u$, of the gas located behind the shock wave front is important in evaluating the energies, and it is always less than the propagating speed, $U$, of the wave front. When a shock wave with a pressure profile, $p(t)$, propagates within a 
shock tube with a cross-sectional area, $S$, it induces a particle speed, $u$ and impacts a specimen, then the energy stored in the impinging gas during element time, $d t$, is equivalent to $p(t) * S * u * d t$. Therefore, the total energy can be obtained by integrating $p(t) * S * u * d t$ with respect to time. The formulas for $E_{\text {incident }}$ and $E_{\text {reflected }}$ are as follows,

$$
\begin{aligned}
& E_{\text {incident }}=\int\left[p_{1}(t) * S * u_{1}\right] d t \\
& E_{\text {reflected }}=\int\left[p_{2}(t) * S * u_{2}\right] d t
\end{aligned}
$$

where, $p_{1}(t)$ is the incident pressure profile, $u_{1}$ is the particle speed behind the incident shock front, $p_{2}(t)$ is the reflected pressure profile, and $u_{2}$ is the particle speed behind the reflected shock front. The incident energy, $E_{\text {incident }}$, is the energy stored in the impinging gas, while the reflected energy, $E_{\text {reflected }}$, is the energy stored in the gas after the incident shock wave impacted the specimen.

In Eq. (1) and Eq. (2), the cross sectional area, $S$, of the shock tube is known and the incident and reflected pressure profiles, $p_{1}(t)$ and $p_{2}(t)$, can be measured. The particle velocities, $u_{1}$ and $u_{2}$ behind the incident and reflected shock front can be calculated using the theory of gas dynamics (Courant and Friedrichs, 1948).

Based on the Hugoniot relation of the polytropic gas and the jump conditions for the shock wave, we can derive the following equations (using incident shock process in Fig. 1a as an example),

$$
\frac{p_{1}}{p_{0}}=\left(1+\mu^{2}\right) M_{0}^{2}-\mu^{2}
$$

or 


$$
\frac{p_{0}}{p_{1}}=\left(1+\mu^{2}\right) M_{1}^{2}-\mu^{2}
$$

and

$$
\left(1-\mu^{2}\right)\left(U_{+}-u_{0}\right)^{2}-\left(u_{1}-u_{0}\right)\left(U_{+}-u_{0}\right)=\left(1-\mu^{2}\right) c_{0}^{2}
$$

or

$$
\left(1-\mu^{2}\right)\left(U_{+}-u_{1}\right)^{2}-\left(u_{0}-u_{1}\right)\left(U_{+}-u_{1}\right)=\left(1-\mu^{2}\right) c_{1}^{2}
$$

where, $\mu^{2}=\frac{\gamma-1}{\gamma+1}, \gamma$ is the adiabatic exponent of the gas, $M$ is the Mach number, $M_{1}=\frac{\left|u_{1}-U_{+}\right|}{c_{1}}$ and $M_{0}=\frac{\left|u_{0}-U_{+}\right|}{c_{0}}, U_{+}$and $U_{-}$are the incident and reflected shock front velocities respectively. $p_{0}$ and $p_{1}$ are the pressures at different locations.

In the present experiments, the incident and reflected processes (as shown in Fig.1a and 1b) generate a system of four independent equations and they are defined by Eq. (3) and Eq. (4). There are twelve parameters in these equations namely: $p_{0}$, $p_{1}, p_{2}, u_{0}, u_{1}, u_{2}, c_{0}, c_{1}, c_{2}, U_{+}, U_{-}$and $\gamma$. Note $u_{0}$ is zero, $c_{0}$ is $340 \mathrm{~m} / \mathrm{s}$ (speed of sound in air), the adiabatic exponent, $\gamma$, is a gas constant and $p_{0}, p_{1}, p_{2}, U_{+}, U_{-}$ can be measured. Therefore, there exist only four unknown parameters, $u_{1}, u_{2}, c_{1}$ and $c_{2}$. The particle speeds behind the shock wave can be solved explicitly as eq. (5).

$$
\begin{gathered}
u_{1}=\frac{\left(1-\mu^{2}\right)\left(U_{+}^{2}-c_{0}^{2}\right)}{U_{+}} \\
u_{2}=\frac{\left(1-\mu^{2}\right)\left(U_{+}^{2}-c_{0}^{2}\right)}{U_{+}}+\left(1-\mu^{2}\right)\left[\frac{\left(1-\mu^{2}\right)\left(U_{+}^{2}-c_{0}^{2}\right)}{U_{+}}+U_{-}\right]\left[\frac{\left(1+\mu^{2}\right)}{\left(p_{2} / p_{1}\right)+\mu^{2}}-1\right]
\end{gathered}
$$


By assuming these particle speeds to be constant during the shock wave loading process, the incident and reflected energies can then be calculated by substituting Eq. (5) into Eq. (1) and (2).

\subsection{The Deformation Energy}

The calculation of the deformation energy is based on the reflected shock wave pressure profile and the high-speed deflection images. The main idea is to obtain the deflection-time data from the high-speed deflection images and the force-time data from the reflected pressure profile. Combining the deflection-time data and the forcetime data will result in force-deflection data. Then the deformation energy can be obtained by integrating the force-deflection data.

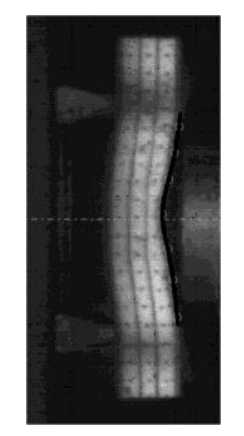

(a) Curve fitting

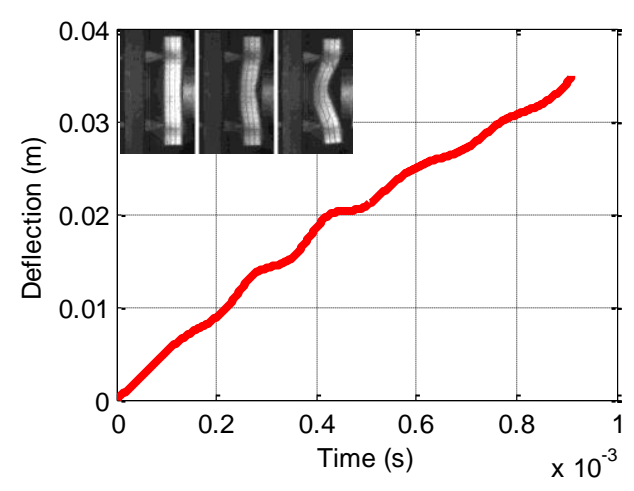

(b) Mid-point deflection-time curve

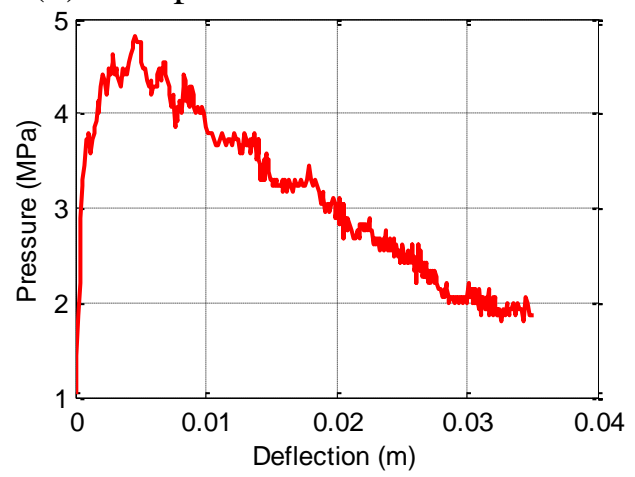

(c) Mid-point pressure-deflection curve

Fig. 2 Deflection based on high-speed images and pressure profile 
The measurement of the deflection is the most important step in this energy calculation. Since the force is only applied on the front face of the specimen, the deflection of the front face of the specimen is what we need. As shown in Fig. 2a, seven points were chosen along the profile of the front face of the specimen in the high-speed images, and a spline curve fitting was applied to match the shape of the front face. After calibrating the distance and choosing the reference point, we can calculate the deflection of every point on the front face. Thus, the deflection-time data can be obtained. Fig. $2 \mathrm{~b}$ shows the typical deflection-time data obtained from this process. By assuming that the pressure applied on the shock area is uniform and combining the pressure-time data and the deflection-time data, the pressure-deflection profile can be obtained, as shown in Fig. 2c. Therefore, the deformation energy $\left(E_{\text {deformation }}\right)$ can be calculated by integrating the pressure-deflection profile of every point inside the shock area. The formula is as follows:

$$
E_{\text {deformation }}=\oint_{S_{\text {ube }}}\left(\int p_{2}(t) d l_{\text {deflection }}\right) d S
$$

\section{Material and Specimen}

\subsection{Skin and Core Materials}

The skin materials that were utilized in this study were E-glass Vinyl-Ester (EVE) composites. The woven roving E-glass fibers of the skin material were placed in a quasi-isotropic layout $[0 / 45 / 90 /-45]_{\mathrm{s}}$. The fibers were made of the $0.61 \mathrm{~kg} / \mathrm{m}^{2}$ areal density plain weave. The resin system used was Ashland Derakane Momentum 8084 and the front skin and the back skin consisted of identical layup and materials.

The core materials used in the present study were Corecell ${ }^{\mathrm{TM}} \mathrm{A}$-series styrene acrylonitrile (SAN) foams, which were manufactured by Gurit SP Technologies 
specifically for marine sandwich composite applications. The three types of Corecell $^{\mathrm{TM}}$ A-series foam that were used in the present study were A300, A500, and A800. Table 1 lists the important material properties of the three foams from the manufacturer's data (http://www.gurit.com).

The cell structures for the three foams were very similar and the only difference appears in the cell wall thickness and node sizes, which accounted for the different densities of the foams.

Table 1. Material properties for foam core (http://www.gurit.com)

\begin{tabular}{cccc}
\hline & $\begin{array}{c}\text { Nominal Density } \\
\mathrm{kg} / \mathrm{m}^{3}\end{array}$ & $\begin{array}{c}\text { Compressive Modulus } \\
\mathrm{MPa}\end{array}$ & $\begin{array}{c}\text { Shear Elongation } \\
\%\end{array}$ \\
\hline A300 & 58.5 & 32 & $62 \%$ \\
A500 & 92 & 64 & $69 \%$ \\
A800 & 150 & 117 & $50 \%$ \\
\hline
\end{tabular}

\subsection{Sandwich Panels with Stepwise Graded Core Layer Arrangement}

The Vacuum Assisted Resin Transfer Molding (VARTM) process was utilized to fabricate the sandwich specimens. During the VARTM process, the sandwich specimens were infused under the same conditions, i.e. temperature, humidity, and vacuum pressure $(760 \mathrm{mmHg}(1 \mathrm{~atm}))$, with the same volume of resin. For the core, each layer of foam was $12.7 \mathrm{~mm}$ thick. The overall dimensions for the samples were $102 \mathrm{~mm}$ wide, $254 \mathrm{~mm}$ long, and $48 \mathrm{~mm}$ thick. The foam core itself was $38 \mathrm{~mm}$ thick, while the skin thickness was $5 \mathrm{~mm}$. The average areal density of the samples was $19.02 \mathrm{~kg} / \mathrm{m}^{2}$.

Two types of sandwich specimens with different core layer gradation were studied (as shown in Fig. 3a). Configuration 1 consisted of a core gradation of 
A300/A500/ A800 (low / middle / high density), and configuration 2 consisted of a core gradation of A500/A300/A800 (middle / low / high density). With these configurations it should be noted that the first core layer was the one first subjected to the shock wave loading. An actual sample can be seen in Fig. $3 b$.

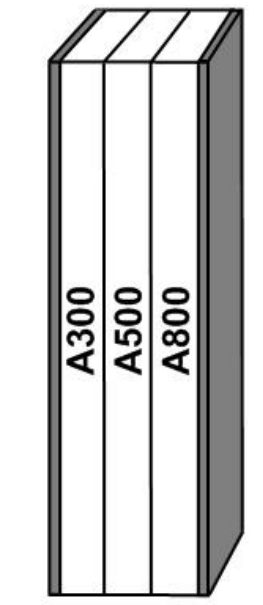

Configuration 1

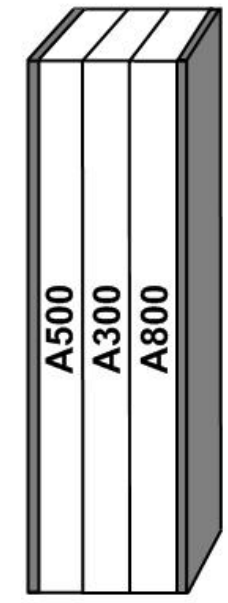

Configuration 2

(a) Two types specimens

Fig. 3 Specimen Configuration

\section{Experimental Set-up and Procedure}

\subsection{Modified Split Hopkinson Pressure Bar with Hollow Transmission Bar}

A Split Hopkinson Pressure Bar (SHPB) is the most common device for measuring dynamic constitutive properties of materials. Due to the low-impedance of Corecell $^{\mathrm{TM}}$ foam materials, dynamic experiments for the core materials were performed with a modified SHPB device with a hollow transmission bar to increase the transmitted signal intensity. A sketch of the modified SHPB device and typical pulse profiles were given in Fig. 4. It had a $304.8 \mathrm{~mm}$-long striker, $1600 \mathrm{~mm}$-long incident bar and $1447 \mathrm{~mm}$-long transmission bar. All of the bars were made of a 6061 aluminum alloy. The nominal outer diameters of the solid incident bar and hollow 
transmission bar were $19.05 \mathrm{~mm}$. The hollow transmission bar had a $16.51 \mathrm{~mm}$ inner diameter. At the head and at the end of the hollow transmission bar, end caps made of the same material as the bar were pressure fitted into the hollow tube. By applying pulse shapers, the effect of these end caps on the stress waves can be minimized. The details of the analysis and derivation of equations for analysis of experimental data can be found in Chen's paper (1998).

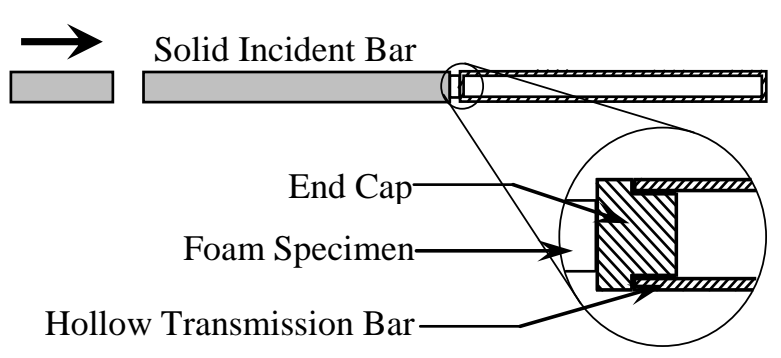

(a) Modified SHPB device

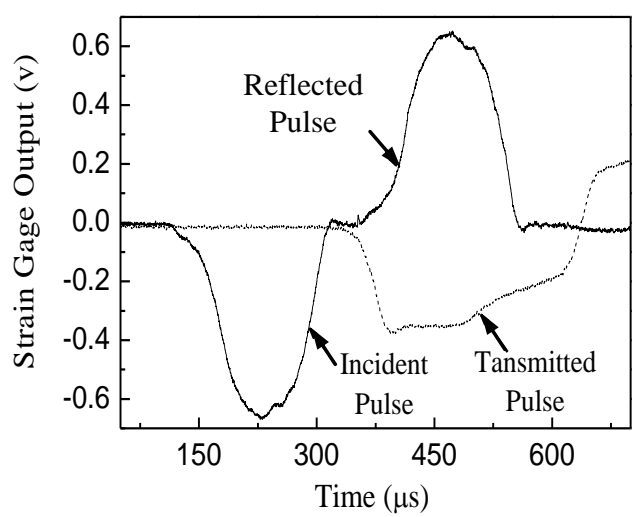

(b) Typical pulse profiles

Fig. 4 Sketch of modified SHPB device with hollow transmission bar and typical

\subsection{Shock Tube} pulse profiles

A shock tube apparatus was utilized to obtain the controlled blast loading (Fig. 5a). It had an overall length of $8 \mathrm{~m}$, consisting of a driver, driven and muzzle section. The high-pressure driver section and the low pressure driven section were separated by a diaphragm. By pressurizing the high-pressure section, a pressure difference across the diaphragm was created. When this difference reached a critical value, the diaphragms ruptured. This rapid release of gas created a shock wave, which travelled down the tube to impart dynamic loading on the specimen.

Fig. 5b showed detailed dimensions and locations of the muzzle, specimen, supports and the pressure sensors (PCB102A). The sensors were mounted at the end of 
the muzzle section to measure the pressure profiles during the experiment. The final muzzle diameter was $0.0762 \mathrm{~m}$. The distance between the two sensors was $0.16 \mathrm{~m}$ and the distance between the second sensor and the end of the muzzle was $\sim 0.02 \mathrm{~m}$. The specimen was placed in the support fixture, which ensured simply supported boundary conditions with a $0.1524 \mathrm{~m}$ span. The front face of the specimen was normal to the axis of the shock tube and had a $\sim 1.6 \mathrm{~mm}$ initial gap to the muzzle end.

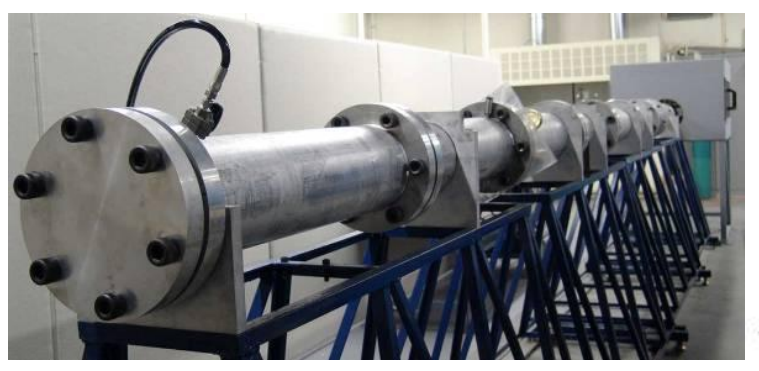

(a) Shock tube

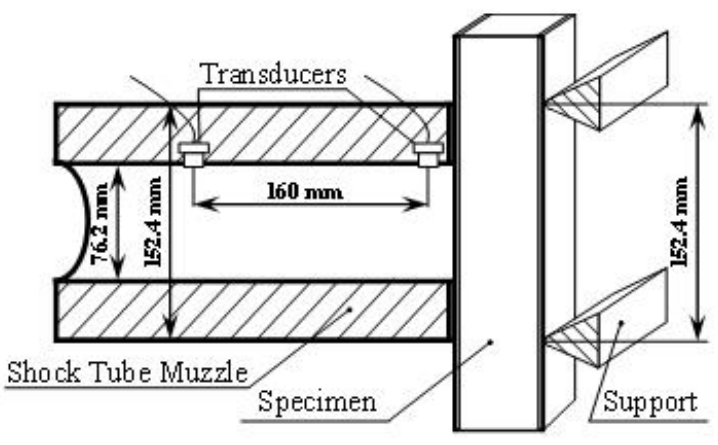

(b) Detail dimensions of the muzzle

Fig. 5 Shock tube apparatus

\subsection{Experimental Procedure and Parameters}

In the present study, a simply stacked diaphragm of 5 plies of $0.254 \mathrm{~mm}$ mylar sheets with a total thickness of $1.270 \mathrm{~mm}$ was utilized to generate an impulse loading on the specimen with an incident peak pressure of approximately $1 \mathrm{MPa}$ and a wave speed of approximately $1000 \mathrm{~m} / \mathrm{s}$. For each configuration, at least three samples were tested. A high-speed digital camera, IMACON 200, was used to capture the real-time side-view deformation of the specimen. With an inter-frame time of $70 \mu$ s and an exposure time of 700 ns, approximately 14 frames could be obtained. Fig. 6 shows the experimental set-up. 


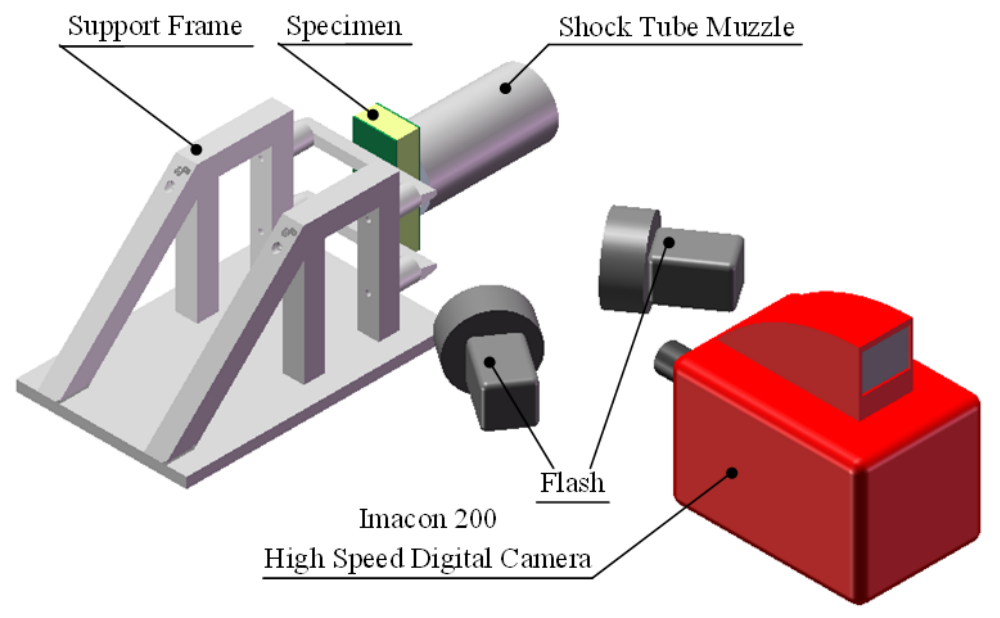

Fig. 6 Experimental set-up

Fig. 7a shows the pressure profile associated with the incident pulse in the absence of a target. This pressure profile was used in calculating the total incident energy. Fig. $7 \mathrm{~b}$ shows the reflected pressure profile obtained by the transducer located at the muzzle end and this pressure profile was used in the calculation of reflected energy as well as the deformation energy of the specimen.

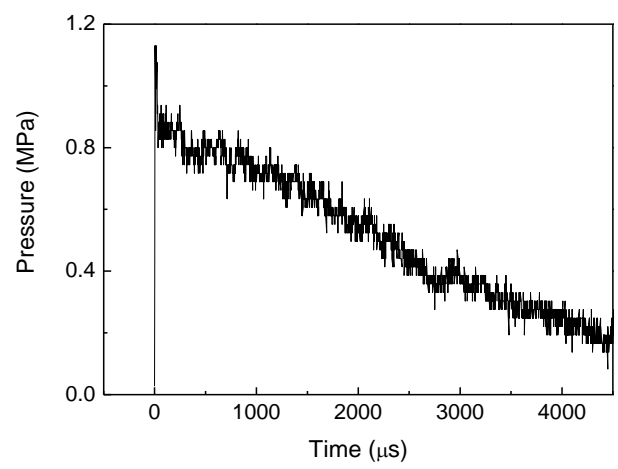

(a) Incident Pressure profile

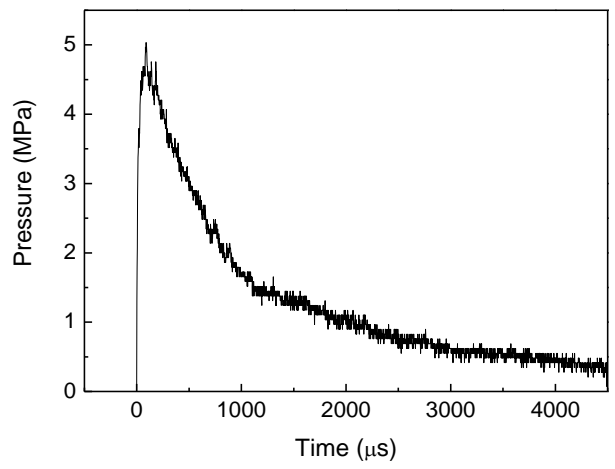

(b) Reflected pressure profile

Fig. 7 Shock wave pressure profiles 


\section{Experimental Results and Discussion}

\subsection{Dynamic Behavior of Core Materials}

The three types of Corecell ${ }^{\mathrm{TM}} \mathrm{A}$ foams have different quasi-static and dynamic behaviors. For the same type of Corecell ${ }^{\mathrm{TM}} \mathrm{A}$ foam, the material behavior under high strain rate loading is significantly different from its behavior under quasi-static loading.

Fig. 8 shows the quasi-static and high strain-rate behavior of the different types of Corecell $^{\mathrm{TM}}$ A foams. For quasi-static behavior, the stress-strain curves showed three deformation regions. The first one was the elastic region; the second was the plateau stress region and the third was the densification region. For high strain rate behavior, the stress-strain curves also showed elastic and plateau stress regions though the strain did not reach the densification region. The plateau stress regions for both instances had a large strain range. This showed the high energy absorption ability of these foams under low stress levels.

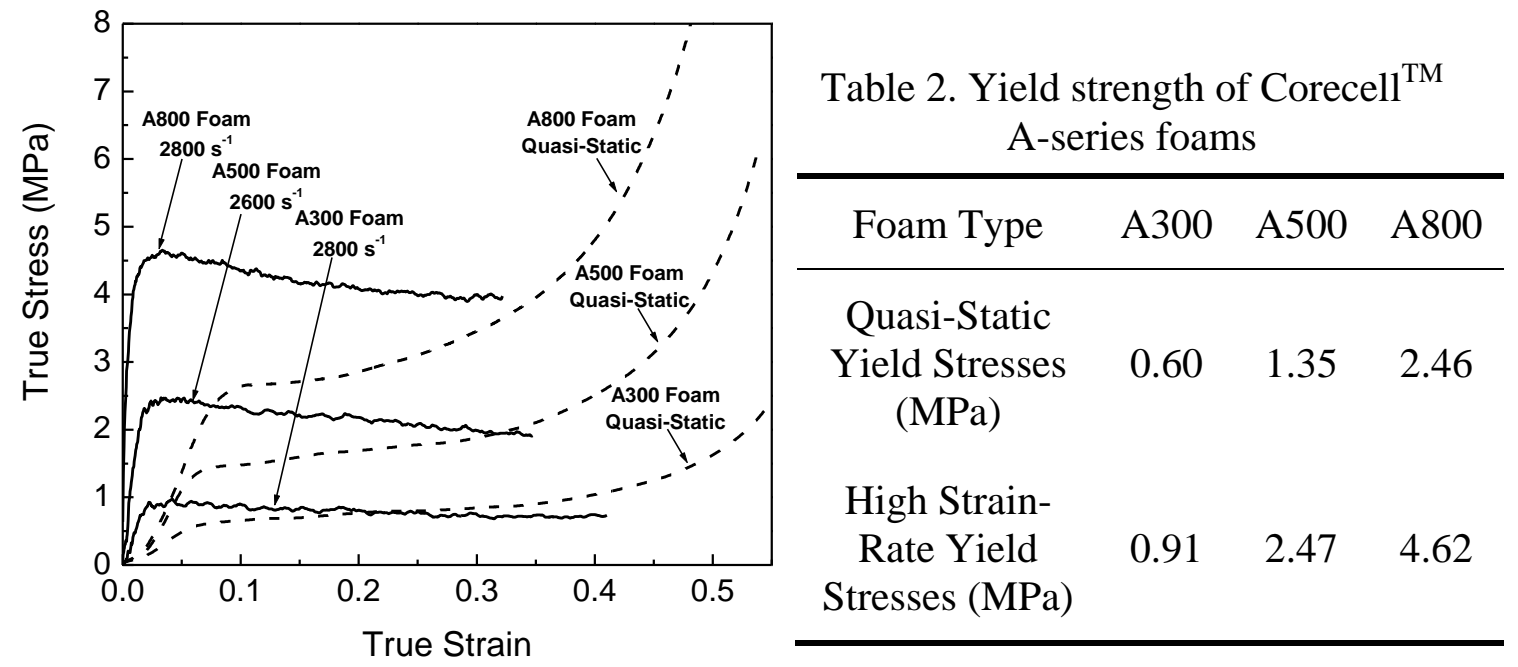

Fig. 8 Quasi-static and high strain-rate behaviors of different types of Corecell ${ }^{\mathrm{TM}}$ A Foams 
As seen in Fig. 8, the quasi-static and dynamic stress-strain responses had an obvious trend for the different types of foams. Lower density foam has a lower strength and stiffness, as well as a larger strain range for the plateau stress.

The high strain-rate yield stresses and plateau stresses were much higher than the quasi-static ones for the same type of foam. Table 2 showed the quasi-static and high strain-rate yield stresses. The dynamic strength of A500 and A800 increased approximately $100 \%$ in comparison to their quasi-static strength, while A300 increased approximately 50\%. The high yield stresses and long stress plateaus indicated that these foams can bear higher stresses and absorb larger amounts of energy. Therefore, they showed great potential in being used as core materials in sandwich structures subjected to high intensity blast loading.

\subsection{Blast Response of Sandwich Composites with Stepwise Graded Core}

\subsubsection{Real-time Deformation}

The real-time observations of the transient behavior of configuration 1 (A300/A500/A800) and configuration 2 (A500/A300/ A800) under shock wave loading were shown in Fig. 9 and Fig. 10 respectively. The shock wave propagated from the right side of the image to the left side and some detailed deformation mechanisms were pointed out in the figures.

For configuration 1, as shown in Fig. 9, the first core layer subjected to the shock wave was A300 and the core gradation was from the foam of least density and lowest strength to the foam of highest density and highest strength. 


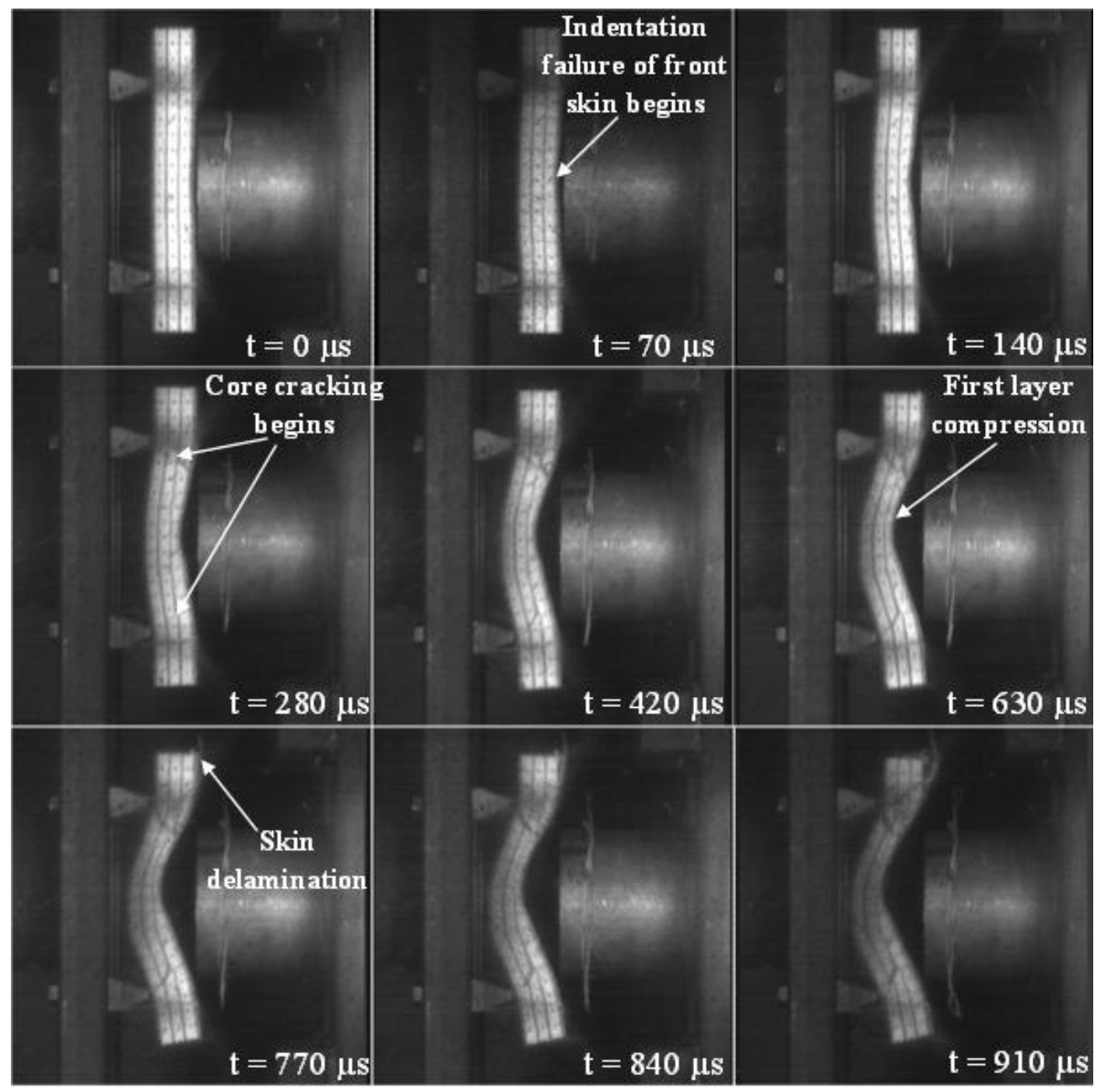

Fig. 9 Real-time side view images of configuration 1 (A300/A500/A800) under shock loading

In this case, two deformation mechanisms were observed during the panel deflection: core compression and global bending. The indentation failure of the front skin can be observed at $\mathrm{t}=70 \mu \mathrm{s}$. Core compression of the A300 foam, the first core layer of gradation, can initially be observed at $140 \mu \mathrm{s}$. At this time there is no compression in the other two core layers of foam. Due to the compression of the foam, the high dynamic pressure applied to the front skin was substantially weakened by the time it reached the back skin. The measurements showed that at $t=420 \mu \mathrm{s}$ and onward 
the central deflection of the A300 foam was approximately $25 \%$ more than that of the A500 and A800 foams. This deflection can be directly related to the density of the A300 foam and its compressive strength. The double-winged deformation shape showed that the core of the sandwich structure was under intense shear loading. The onset of core failure, where core cracking begins, was observed at $t=280 \mu \mathrm{s}$ and the initial separation / delamination of the front skin from the core was observed at $\mathrm{t}=770$ $\mu \mathrm{s}$; this indicated relatively weak adhesion. Even though the onset of core failure began at $\mathrm{t}=280 \mu \mathrm{s}$, complete core collapse and failure was not observed in this configuration.

In configuration 2, as shown in Fig. 10, A500 was the first core layer subjected to the shock wave and the core gradation began with the foam of middle density and middle strength, next the foam of least density and lowest strength, and then the highest density and highest strength foam.

The images in Fig. 10 show that indentation failure of the front skin began at $t=$ $70 \mu \mathrm{s}$. Also note that the central core compression was not as prominent in this sandwich as can be seen in configuration 1. The initial separation / delamination of the core began at $\mathrm{t}=350 \mu \mathrm{s}$ and was located between the A500 and A300 foams. The onset of core failure, where core cracking began, can be seen at $t=140 \mu$ s and the onset of complete collapse of the core initiates at $t=490 \mu \mathrm{s}$, where the core cracking had traveled completely through the core. In this case, the only deformation mechanism observed was global bending. 


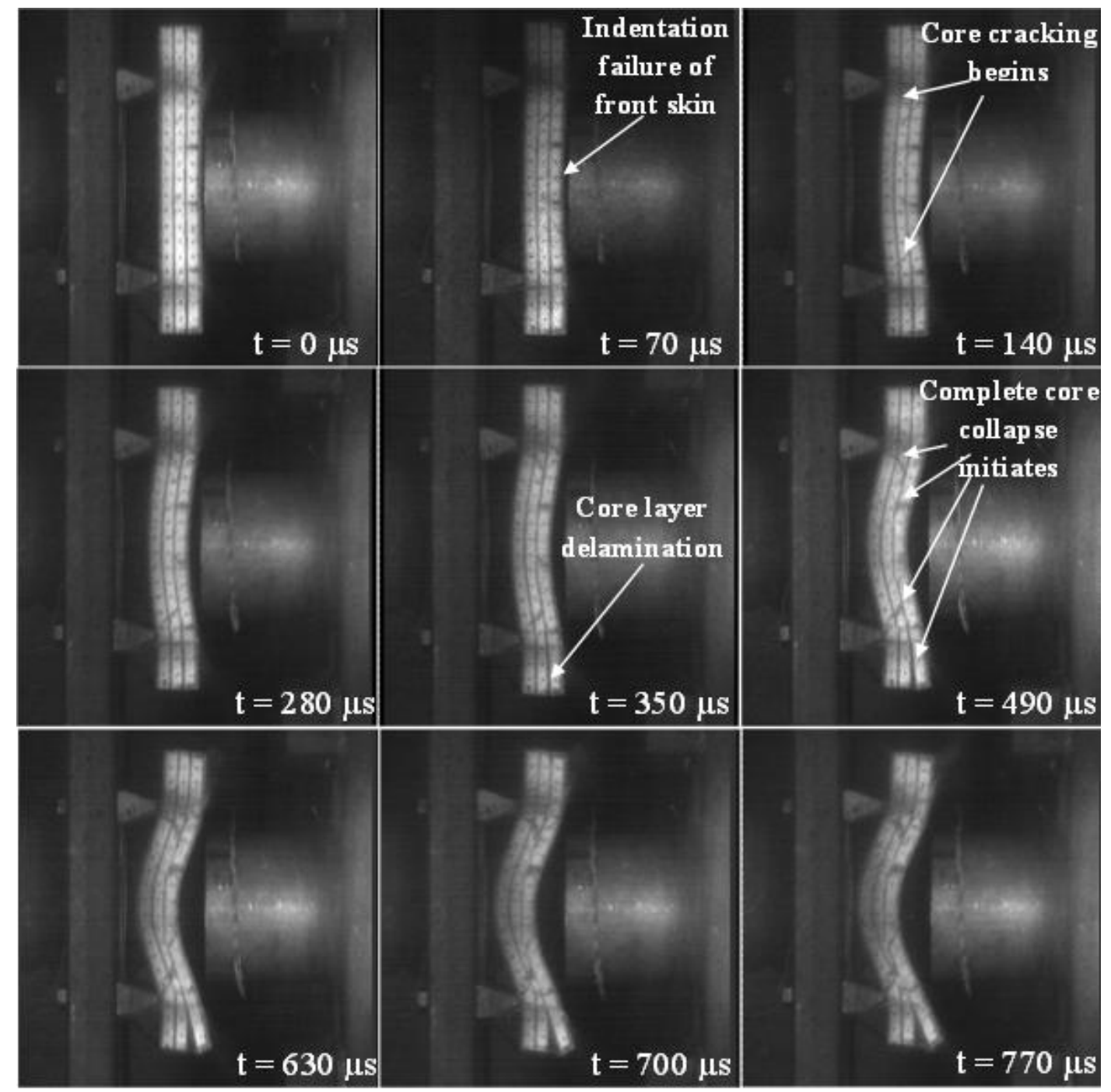

Fig. 10 Real-time side view images of configuration 2 (A500/A300/A800) under shock loading

The major failure mechanism in configuration 2 was progressive damage of the core and the sandwich, which initiated at the back skin and was evident in Fig. 10. This crack became a large inclined crack and propagated through the core from the back skin to the front skin. By $t=490 \mu$ s the crack had extended completely through the core and delamination between the A300 and A500 foam was very prominent. Also at this time, many cracks were visible in the core which is followed by a rapid crushing of the core and catastrophic failure of the sandwich structure. This showed 
that configuration 2 cannot withstand the applied shock wave pressure, which had a peak value of $\sim 4.83 \mathrm{MPa}$.

Contrary to the case of configuration 2 the real-time deformation sequences observed in Fig. 9 for configuration 1 indicated that the major failure mechanism was core compression. The results showed that the core lay-up improved the overall performance of the structure. The onset of core failure took twice as long to be visible in this configuration as opposed to configuration 2 and no complete core collapse was evident. Even though delamination did occur, it was between the facesheet and foam core only. Overall configuration 1 outperformed configuration 2, and this was related to the dynamic constitutive behaviors of the foam core materials and the order of the core layer arrangements. For configuration 1, the strength of the core layers increase monotonously from the front facesheet to the back facesheet. Due to the low yield stress of the first core layer, A300, under dynamic loading, core compression occurred before the sandwich panel exhibited any bending (indentation failure in Fig. 9) and the core layers were compressed layer by layer. For configuration 2 , the strength of the core layers did not increase monotonously from the front facesheet to the back facesheet. Here the first core layer, A500, had higher strength in comparison to A300 foam. These factors neutralized the core compression even though the core materials were identical. Thus bending occurred before the sandwich panel exhibited core compression. 


\subsubsection{Deflection}

The mid-point deflections of each graded sandwich panel and all of its constituents were obtained from the high-speed images. The deflection of the front face (front skin), interface 1 (between first and second core layer), interface 2 (between second and third core layer), and back face (back skin) for configuration 1 and configuration 2 were plotted in Fig. 11 and Fig. 12. It can be seen in Fig. 11 for configuration 1 that the front face deflects to $\sim 33 \mathrm{~mm}$ at $\sim \mathrm{t}=840 \mu \mathrm{s}$, which was approximately $25 \%$ more than the other three constituents. Note that the difference between the front face (skin) and interface 1 was the A300 foam, which was the weakest foam in three types of foams, and almost all compression occurs here $(\sim 7$ $\mathrm{mm})$.

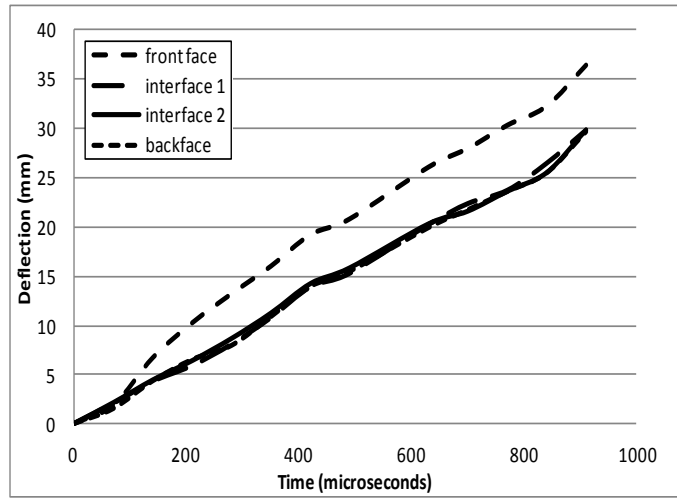

Fig. 11 Deflection of A300/A500/A800

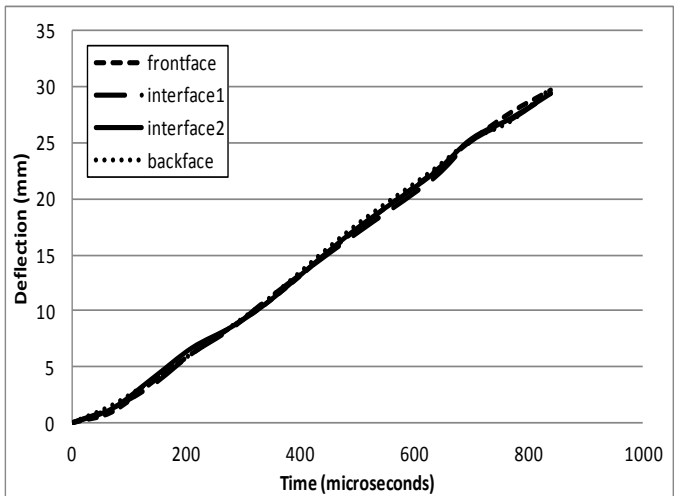

Fig. 12 Deflection of A500/A300/A800

On the contrary, all of the constituents of configuration 2 deflect in the same manner (shown in Fig. 12). This showed almost no obvious compression, even though the core foams of configuration 1 and configuration 2 were identical, but in a different gradation. Also this graded sandwich panel only deflected to $\sim 29 \mathrm{~mm}$ at $\sim \mathrm{t}=840 \mu \mathrm{s}$. 


\subsubsection{Post-mortem Analysis}

The damage patterns in the graded sandwich composites after the shock event occurred were visually examined and recorded using a high resolution digital camera and were shown in Fig. 13.

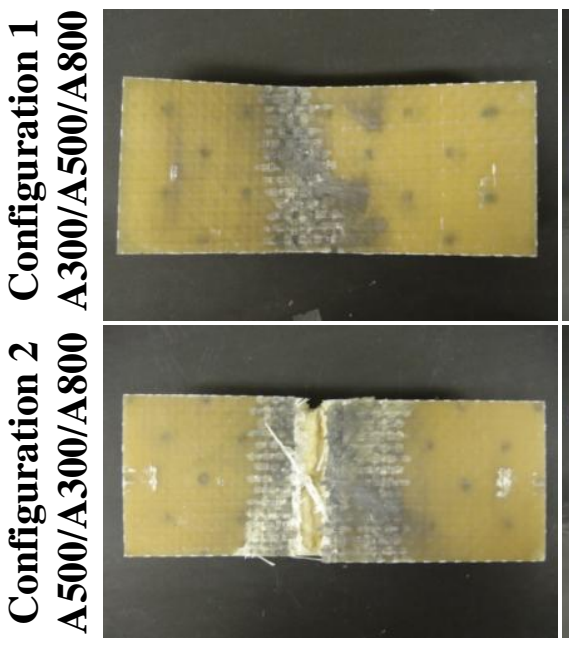

(a) Front facesheet (blast side)

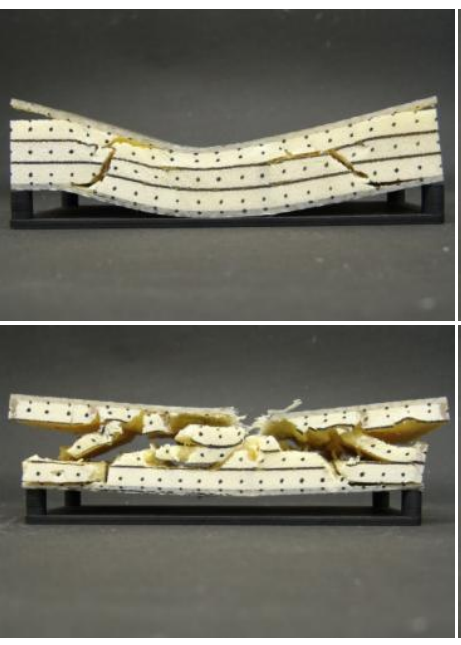

(b) Foam core

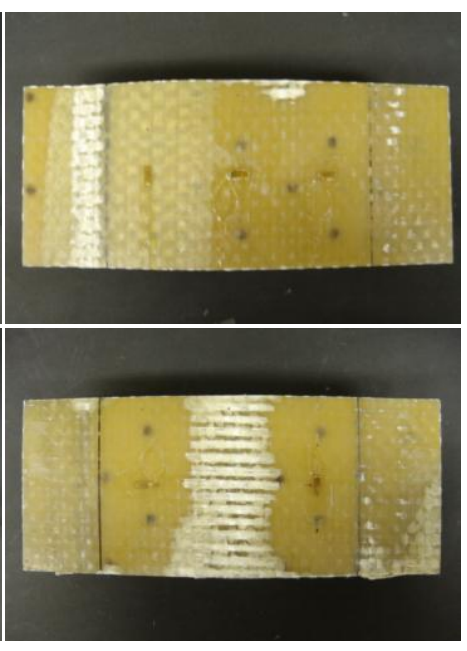

(c) Back facesheet

Fig. 13 Visual examination of sandwich composites after being subjected to high intensity blast load

When configuration 1 was subjected to the highly transient loading, the damage was confined to the area where the supports were located in the shock tube and core cracking was visible in these two areas. Delamination was visible between the front skin and the foam core, as well as the back skin and the foam core. The core compression can be seen clearly and distinctively in the A300 foam.

Microscopic analysis of the failure and compression observed in configuration 1 was done using a Nikon SMZ microscope. Pre and post-blast core cell structures for the three layers of gradation were shown in Fig. 14. Note the heavy amount of compression seen in the A300 foam core cell structure. Also the cell structure for the 
A500 foam did indeed compress, but not nearly as much as can be seen in the A300 foam. Likewise, the A800 foam core cell structure did compress, but only minimally.

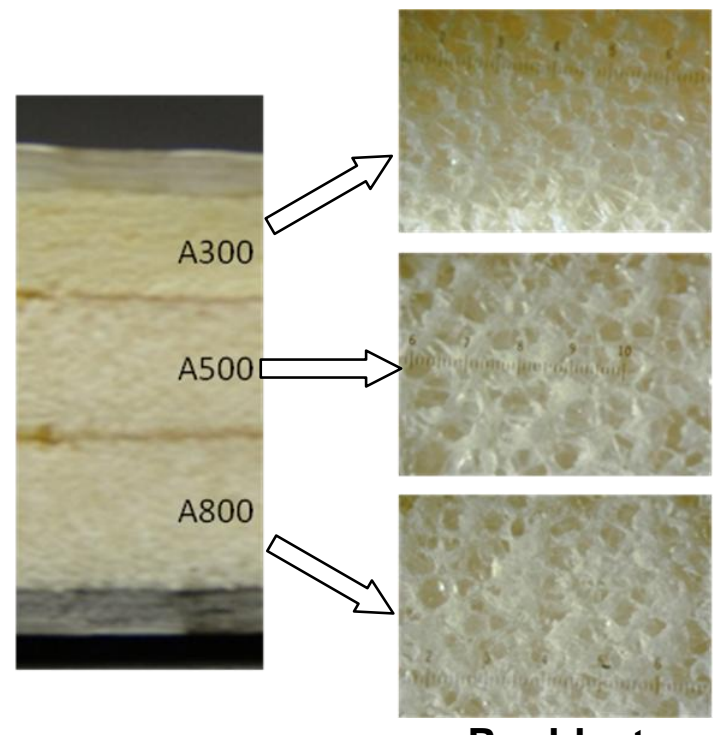

Pre-blast
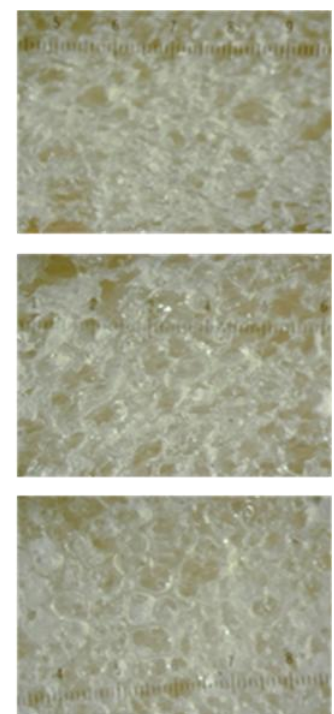

Post-blast

Fig. 14 The failure and compression of core foam cell microstructure in configuration 1

Unlike the damage visible in configuration 1, configuration 2 suffered catastrophic damage as shown in Fig. 13. The core of the sandwich disintegrated and the front skin (blast side) of the sandwich fractured into two pieces at the midsection. The back skin showed an extensive amount of fiber delamination in the central region as well.

Fig. 15 shows the details of the failure modes in configuration 2 . The photograph shows a flat segment located at the incident face indicating that the stresses were released in this region. The cracks observed on both sides of the flat segment do not resemble those caused by the bending shear stresses which were typical near the supports. The detailed macroscopic images of the local cracks and delamination 
surfaces show that the delamination surfaces exhibit similar material granules as those observed in a pure tension test.
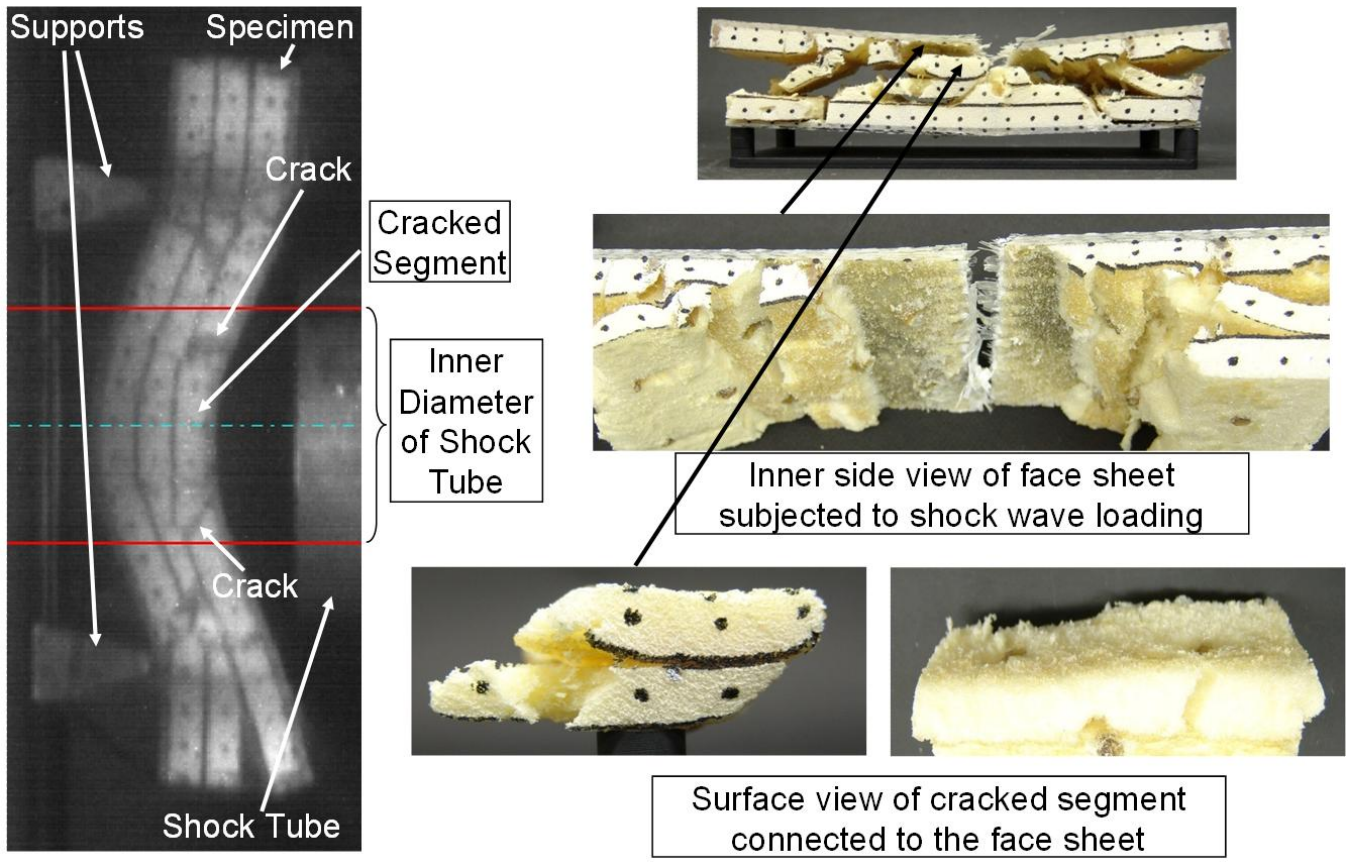

Fig. 15 The details of the failure mode in configuration 2

\subsubsection{Energy Evaluation}

The energies calculated by the methods described in section 2 were shown in Fig. 16 and Fig. 17. With regards to the choice of the adiabatic component, $\gamma$, the following explanation was offered. In the present shock tube experiments, prior to the diaphragm rupturing, one side of the diaphragm was helium (driver side), while the other side of the diaphragm was air (driven side). After the diaphragm ruptured the compressive shock wave travelled in the direction of higher pressure to lower pressure (helium $->$ air). Since the particle speed of the gas (helium) located behind the shock front was less than the speed of the shock front itself, air passed over the shock front and occupied the space located between the gas (helium) and the shock front during 
the propagation of the shock wave. Therefore, by the time the shock wave reached the specimen, the gas located to the front and back side of the shock front were both airs. Thus, the adiabatic exponent of air, $\gamma=1.4$, was used in the energy calculations.

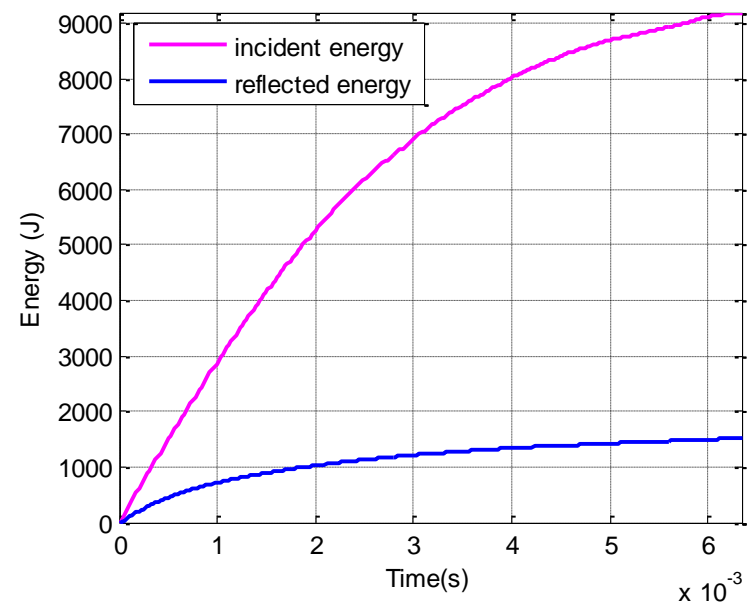

Fig. 16 Incident and reflected energies of configuration 1 (A300 / A500 / A800)

Fig. 16 shows the incident and reflected energy calculated for configuration 1(A300/500/800). The difference between the incident and reflected energies was the total energy lost during the shock wave loading process. It included the energy absorbed by the composite structures, sound, light, heat, rigid body motion and other forms of energy. We defined it as the total amount of energy loss. It can be seen that there is a large amount of energy lost during the shock wave loading process.

The initial gap between the specimen and the muzzle end $(\sim 1.6 \mathrm{~mm})$ increased after the impingement of shock on the specimen as the specimen deformed in a concave manner. The gas leak from this gap did affect the reflected energy calculation as it influenced the reflected pressure drop. Therefore, a fraction of energy was lost due to the gas that escaped from this gap. This lost energy was included in our estimation of the total energy loss. 


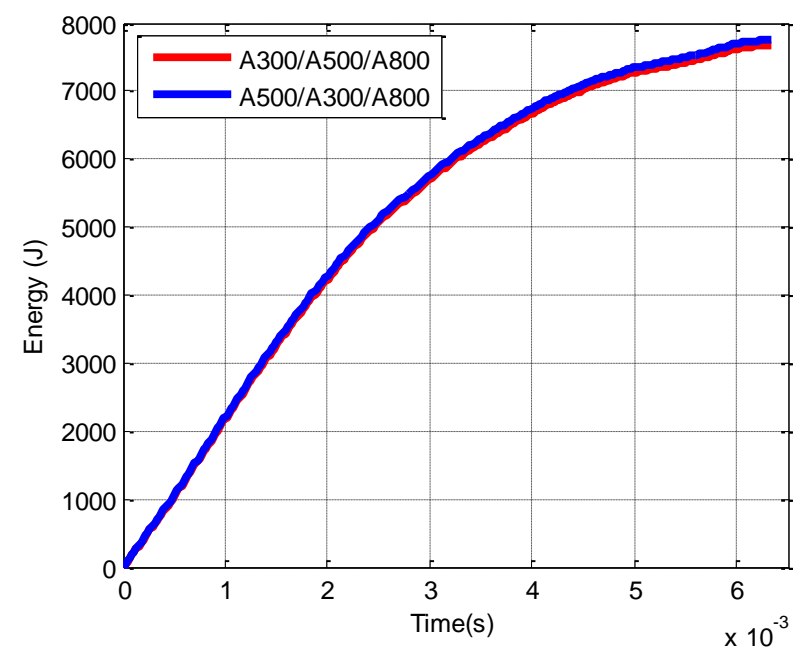

(a) The total energy loss

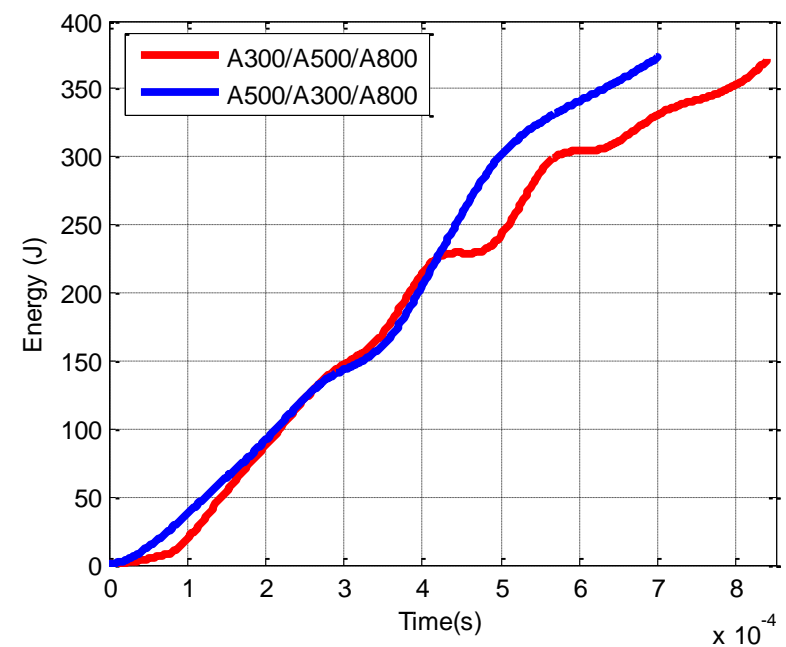

(b) The deformation energy

Fig. 17 A comparison of the total energy loss and a comparison of the deformation energy for two configurations

Fig. 17a compared the total energy loss of configuration 1 (A300/500/800) and configuration 2(A500/300/800), while Fig. 17b compared the deformation energy of configuration 1 and configuration 2 respectively. It can be seen in Fig. 17a that the total energy loss for both configurations was almost identical. The deformation energy of configuration 2(A500/300/800) was a slightly higher than that of configuration 1(A300/500/800). This minimal difference can be ignored due to the error that arose 
when measuring the positions and deflection using the high-speed images. With that said, these two configuration exhibited similar energy transferring properties when subjected to the same input shock wave loading. Configuration 1(A300/500/800) showed no obvious structural collapse while configuration 2(A500/300/800) exhibited total structural collapse. Therefore, it can be concluded that configuration 1(A300/500/800) can withstand a higher blast loading than configuration 2(A500/300/800) and thus overall outperformed configuration 2.

Due to the fact that the deformation energy (Fig. 17b) was much less than the total amount of energy loss (Fig. 17a), it can be concluded that only a small amount of energy was transferred into the sandwich structure. At $0.6 \mathrm{~ms}$, the total energy loss was approximately $1300 \mathrm{~J}$, while the deformation energy was only $\sim 350 \mathrm{~J}$. This indicated that only $\sim 25 \%$ of the total energy lost was transferred into the specimen and most of the energy actually dissipated into other forms of energy (sound, heat, light, rigid body motion and various other forms).

\section{Conclusions}

(1) The dynamic stress-strain response was significantly higher than the quasistatic response for every type of Corecell ${ }^{\mathrm{TM}} \mathrm{A}$-series foam studied. Both quasistatic and dynamic constitutive behaviors of Corecell ${ }^{\mathrm{TM}}$ A-series foams (A300, A500, A800) showed an increasing trend.

(2) The sandwich specimens with two different core arrangements, configuration 1 (A300/A500/A800) and configuration 2 (A500/A300/A800), were subjected to shock wave loading. The overall performance of configuration 1 (A300/A500/A800) was better than that of configuration 2 (A500/A300/A800). 
Large compression was visible in the core when the least density foam (A300) is first in contact with the blast loading. This configuration reduced the dynamic pressures seen on the back facesheet, and thus limited the total amount of damage imparted on the specimen. When using the A500 foam first in contact with the blast loading, the overall deformation process of the sample was completely different. Compression in the core was limited, and thus the specimen showed a heavy amount of damage.

(3) The methods used to calculate the energy of the incident energy, the reflected energy and the deformation energy were proposed and implemented. The difference between the total incident and reflected energy was defined as the total energy loss in the system during the shock loading process. Only a small amount of energy was transferred into the specimens during the shock loading process. The total energy loss in the two configurations as well as their deformation energy was almost identical. Therefore, since configuration 2(A500/A300/A800) showed heavy damage and failure, it can be concluded that overall configuration 1(A300/A500/A800) outperformed configuration 2(A500/A300/A800).

\section{Acknowledgements}

The authors kindly acknowledge the financial support provided by Dr. Yapa D. S. Rajapakse, under Office of Naval Research (ONR) Grant No. N00014-04-1-0268. The authors acknowledge the support provided by the Department of Homeland Security (DHS) under Cooperative Agreement No. 2008-ST-061-ED0002. Authors also thank 
Dr. Stephen Nolet and TPI Composites for providing the facility for creating the materials used in this study.

\section{References}

Apetre, N.A., Sankar, B.V. and Ambur, D.R., 2006. Low-velocity impact response of sandwich beams with functionally graded core. International Journal of Solids and Structures 43 (9) 2479-2496.

Chen, W., Zhang, B., and Forrestal, M.J., 1998. A split Hopkinson bar technique for low-impedance materials. Experiment Mechanics 39 (2) 81-85.

Courant, R. and Friedrichs, K.O., 1948. Supersonic flow and shock waves. Interscience Publishers, New York.

Dharmasena, K.P., Wadley, H.N.G., Xue, Z. and Hutchinson, J.W., 2008. Mechanical response of metallic honeycomb sandwich panel structures to high-intensity dynamic loading. International Journal of Impact Engineering 35 (9) 1063-1074.

Fleck, N.A., Deshpande, V.S., 2004. The resistance of clamped sandwich beams to shock loading. Journal of Applied Mechanics 71 386-401.

http://www.gurit.com (Accessed February 9, 2009).

Li, R.F., Kardomateas, G.A. and Simitses, G.J., 2008. Nonlinear Response of a Shallow: Sandwich Shell With Compressible Core to Blast Loading. Journal of Applied Mechanics-Transactions of the ASME 75 (6) 125-138.

Li, Y., Ramesh, K.T. and Chin, E.S.C., 2001. Dynamic characterization of layered and graded structures under impulsive loading. International Journal of Solids and Structures 38 (34-35) 6045-6061.

McShane, G.J., Radford, D.D., Deshpande, V.S. and Fleck, N.A., 2006. The response of clamped sandwich plates with lattice cores subjected to shock loading. European Journal of Mechanics - A: Solids 25 215-229. 
Nurick, G.N., Langdon, G.S., Chi, Y. and Jacob, N., 2008. Behavior of sandwich panels subjected to intense air blast: part 1- Experiments. Composite Structures, in press.

Radford, D.D., McShane, G.J., Deshpande, V.S. and Fleck, N.A., 2006. The response of clamped sandwich plates with metallic foam cores to simulated blast loading. International Journal of Solids and Structures 44 6101-6123.

Tekalur, S.A., Bogdanovich, A.E. and Shukla, A., 2008. Shock loading response of sandwich panels with 3-D woven E-glass composite skins and stitched foam core. Composite Science and Technology, doi:10.1016/j.compscitech.2008.03.017.

Wadley, H.N.G., Dharmasena, K.P., Chen, Y., Dudt, P., Knight, D., Charette, R. and Kiddy, K., 2008. Compressive response of multilayered pyramidal lattices during underwater shock loading. International Journal of Impact Engineering 35 (9) 11021114.

Xue, Z. and Hutchinson, J.W., 2003. Preliminary assessment of sandwich plates subject to blast loads. International Journal of Mechanical Sciences 45 687-705.

Zhu, F., Zhao, L., Lu, G. and Wang, Z., 2008. Deformation and failure of blast loaded metallic sandwich panels - Experimental investigations. International Journal of Impact Engineering 35 (8) 937-951. 


\title{
CHAPTER 3
}

\section{PERFORMANCE OF FUNCTIONALLY GRADED SANDWICH COMPOSITE BEAMS UNDER SHOCK WAVE LOADING}

by

\author{
Nate Gardner, Erheng Wang and Arun Shukla
}

has been published in Composite Structures (2012, Vol. 94, 1755-1770)

Corresponding Author: Arun Shukla

Dynamic Photo Mechanics Laboratory

Department of Mechanical, Industrial and Systems

Engineering

University of Rhode Island

206 Wales Hall, 92 Upper College Rd

Kingston, RI, 02881, USA

Phone: +1-401-874-2283

Email Address: shuklaa@egr.uri.edu 


\begin{abstract}
The dynamic behavior of sandwich composites made of E-glass Vinyl-Ester (EVE) facesheets and graded Corecell ${ }^{\mathrm{TM}}$ A-series foam was studied using a shock tube apparatus. The foam core was monotonically graded based on increasing acoustic wave impedance, with the foam core layer of lowest wave impedance facing the blast. The specimen dimensions were held constant for all core configurations, while the number of core layers varied, resulting in specimens with one layer, two layer, three layer, and four layers of foam core gradation. Prior to shock tube testing, the quasistatic and dynamic constitutive behavior (compressive) of each type of foam was evaluated. During the shock tube testing, high-speed photography coupled with the optical technique of Digital Image Correlation (DIC) was utilized to capture the realtime deformation process as well as mechanisms of failure. Post-mortem analysis was also carried out to evaluate the overall blast performance of these configurations. The results indicated that increasing the number of monotonically graded foam core layers, thus reducing the acoustic wave impedance mismatch between successive layers, helped maintain structural integrity and increased the blast performance of the sandwich composite.
\end{abstract}

Keywords: Sandwich Structures, Functionally Graded Materials, Acoustic Wave Impedance, Blast Loading, High-Speed Photography

\title{
1. Introduction
}

Sandwich structures have very important applications in the naval and aerospace industry. Due to their construction they have many advantages that include high strength/weight ratio, high stiffness/weight ratio, and energy absorption capabilities. 
Sandwich structures consist of two thin, stiff facesheets, usually the same thickness, separated by a lightweight, thicker core. The facesheets carry almost all of the bending and in-plane loads, while the core helps to stabilize the facesheets and defines the flexural stiffness and out-of-plane shear and compressive behavior. When sandwich structures are subjected to high-intensity impulse loadings, such as air blasts, the core materials play a crucial role in the dynamic behavior and overall structural response. Their properties assist in dispersing the mechanical impulse that is transmitted into the structure, and thus protect anything located behind it [1-3].

Common cores are made of metallic and non-metallic honeycombs, cellular foams, balsa wood, PVC, truss and lattice structures. Extensive research exists in the literature regarding the dynamic response of sandwich structures consisting of the various core materials and geometric structures [3-11]. Dharmasena et al. [3], Zhu et al. [4], and Nurick et al. [5] have tested sandwich structures with a metallic honeycomb core material. Their results indicated that the parameters of core materials can effectively reduce the transmitted impulse and damage of the back facesheet. Radford et al. [6] and Wang et al. [7] have conducted metal foam projectile impact experiments to simulate a blast loading on sandwich structures with metal foam cores. Tagarielli et al. [8] and Atas et al. [9] have investigated the dynamic response of sandwich beams with PVC and balsa wood cores. McShane et al. [10, 11] have investigated the underwater blast response of sandwich composites with a prismatic lattice (Y-frame, corrugated), as well as simulated an air blast, using metal foam projectiles, on sandwich composites with a pyramidal lattice cores. These studies have indicated that advanced sandwich structures can potentially have significant 
advantages over monolithic plates of equivalent mass in absorbing the blast energy, whether in air or underwater.

In recent years, functionally graded materials, where the material properties vary gradually or layer by layer within the material itself, have gained much attention. Hossain et al. [12] have experimentally studied the compressive behavior of a functionally graded foam material system and energy absorption under quasi-static loading conditions. The results indicated stepwise crushing from the lower density to the higher density foams. Kiernan et al. [13] numerically investigated the propagation of a stress wave through a virtual functionally graded foam material (FGFM). It was concluded that the amplitude of a stress wave propagating through a FGFM can be shaped by the gradient functions according to which the foam density varies through the direction of wave propagation. Cui et al. [14] proposed a functionally graded foam model to improve the energy absorption characteristics offered by uniform foams. In this model, the characteristics of the foam (e.g. density) are varied through the thickness. Results indicated that for high energy impacts, increasing the density range can decrease the performance of the graded foams over conventional foams of uniform density. Consequently, decreasing the density range can increase the performance of the graded foams over conventional foams of uniform density.

Since the properties of the layered/graded material can be designed and controlled, they show great potential to be an effective core material for energy absorption and blast mitigation. To date, there have been very little results published regarding the dynamic impact response of sandwich composites with a functionally graded core, and even less regarding the blast response. Li et al. [15] numerically 
examined the response of layered and graded metal-ceramic structures under impulsive loadings. It was concluded that the choice of gradation has a great significance on the impact applications and the particular design can exhibit better energy dissipation properties. Apetre et al. [16] numerically investigated the impact response of sandwich beams with a functionally graded core. Their results showed that a reasonable core design can effectively reduce the shear forces and strains within the structure. Consequently, they can mitigate or completely prevent impact damage on sandwich composites. In the previous work by the authors [17] they investigated the blast resistance of sandwich composites with a discretely layered foam core. They concluded that monotonically increasing the density of the foam core allows for a stepwise compression of the core layers and thus reduces the dynamic pressures imparted on the back facesheet.

Due to the fact that the authors' earlier work [17] was limited to sandwich composites with one specific foam core configuration (i.e. 3 layers of core gradation), the current study will expand upon the previous work and investigate the influence of the number of monotonically graded foam core layers (based on increasing acoustic wave impedance) on the overall blast response of the sandwich structure. For this investigation a series of four (4) different foam core layer arrangements were studied, which consisted of one layer, two layers, three layers, and four layers of gradation respectively. The purpose of this investigation was to study the role of material interfaces and the acoustic wave impedance mismatch between consecutive core layers on the dynamic behavior and blast response of the structure, and the results will help provide an optimal core design to help mitigate the high-intensity impulse 
loading. More importantly, the rationale for this series was based off of creating a sandwich composite structure with enough foam core layers to reduce impedance mismatch between successive layers, therefore creating a theoretical "continuous" gradation. The quasi-static and dynamic constitutive behaviors of the foam core materials were first studied using a modified SHPB device with a hollow transmitted bar. The sandwich composites with different core layer arrangements were then fabricated and subjected to shock wave loading generated by a shock tube. All of the sandwich composites have an identical core thickness and overall specimen geometry, but a different number of core layers and overall areal densities. The shock pressure profiles and real-time deformation images were carefully analyzed to reveal the failure mechanisms of these sandwich composites. Digital Image Correlation (DIC) analysis was implemented to investigate the real-time deflection, in-plane strain and velocity of the back face of the specimens. Post-mortem analysis was also carried out to evaluate the overall blast performance of these sandwich structures.

\section{Material and Specimen}

\subsection{Skin and Core Materials}

The skin materials that were utilized in this study are E-glass Vinyl-Ester (EVE) composites. The woven roving E-glass fibers of the skin material were placed in a quasi-isotropic layout $[0 / 45 / 90 /-45]_{\mathrm{s}}$. The fibers were made of the $0.61 \mathrm{~kg} / \mathrm{m}^{2}$ areal

density plain weave. The resin system used was Ashland Derakane Momentum 8084, with the front and back skins (facesheets) consisting of identical layups and materials. Fig. 1 shows a schematic of the sandwich composite with skin and core materials. 

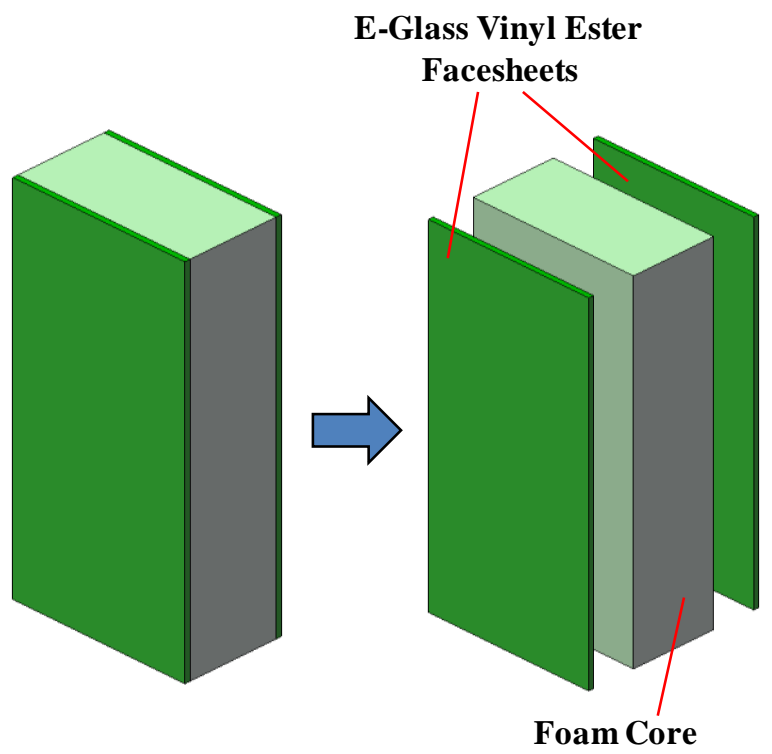

Fig. 1 Schematic of sandwich composite with skin and core

The core materials used in the present study are Corecell ${ }^{\mathrm{TM}} \mathrm{A}$-series styrene acrylonitrile (SAN) foams, which are manufactured by Gurit SP Technologies specifically for marine sandwich composite applications. The four types of Corecell ${ }^{\mathrm{TM}}$ A-series foam that were used in present study were A300, A400, A500, and A800. Table 1 lists important material properties of the four foams from the manufacturer's data [18], as well as the material properties of the facesheet. The material properties of the facesheet and the core materials were determined using proper ASTM standards, D 3410 and D 1621 respectively, as well as Rule of Mixtures (ROM) formula (transverse material properties).

In Table 1, the A300 foam has the lowest nominal density ( $\rho$ ), as well as compressive modulus (E) of the four foams, followed by the A400, A500 and A800 foams respectively. Since both the nominal density and the compressive modulus are increasing from A300 to A800 foam, the one-dimensional acoustic wave impedance (Z) also increases, and shows the following relationship, 


$$
\begin{gathered}
Z=\rho C=\rho \sqrt{E / \rho} \\
Z_{A 800}>Z_{A 500}>Z_{A 400}>Z_{A 300}
\end{gathered}
$$

The cell structures for the four foams are very similar and the only difference appears in the cell wall thickness and node sizes, which accounts for the different densities of the foams. The SEM images of the cell microstructures can be seen in Fig.

2.

Table 1. Quasi-static material properties of foam core [18] and EVE facesheet

\begin{tabular}{ccccc}
\hline & $\begin{array}{c}\text { Nominal } \\
\text { Density, } \boldsymbol{\rho} \\
\left(\mathrm{kg} / \mathrm{m}^{3}\right)\end{array}$ & $\begin{array}{c}\text { Compressive } \\
\text { Modulus, } \mathbf{E} \\
(\mathrm{MPa})\end{array}$ & $\begin{array}{c}\text { Compressive } \\
\text { Strength, } \boldsymbol{\sigma}_{\mathbf{y}} \\
(\mathrm{MPa})\end{array}$ & $\begin{array}{c}\text { Acoustic Wave } \\
\text { Impedance } \\
\left(\mathrm{kg} / \mathrm{m}^{2} \mathrm{~s}\right)\end{array}$ \\
\hline $\mathbf{A 3 0 0}$ & 58.5 & 32 & 0.5 & $4.3 \times 10^{4}$ \\
$\mathbf{A 4 0 0}$ & 69 & 41 & 0.6 & $5.3 \times 10^{4}$ \\
$\mathbf{A 5 0 0}$ & 92 & 64 & 0.9 & $7.7 \times 10^{4}$ \\
$\mathbf{A 8 0 0}$ & 150 & 117 & 2.1 & $13.2 \times 10^{4}$ \\
$\begin{array}{c}\text { E-glass } \\
\text { Vinyl-Ester } \\
\text { Composite }\end{array}$ & 1800 & $\begin{array}{c}13600 \\
{[\text { longitudinal] }} \\
6200\end{array}$ & 220 & $\begin{array}{c}4.9 \times 10^{6} \\
{[\text { longitudinal] }} \\
\text { [transverse] }\end{array}$ \\
\hline
\end{tabular}

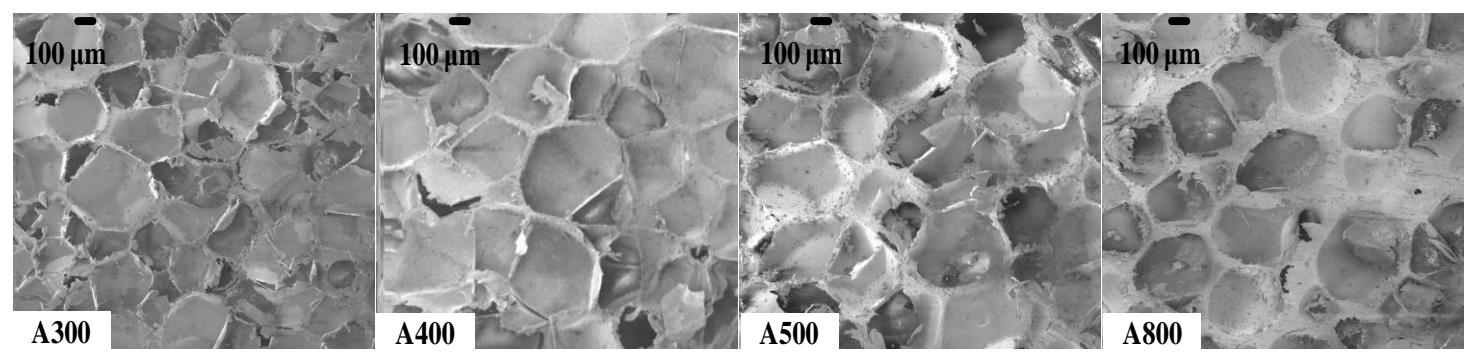

Fig. 2 Cell microstructure of foam core layers [19] 


\subsection{Sandwich Panels with Functionally Graded Core Layer Arrangement}

The Vacuum Assisted Resin Transfer Molding (VARTM) process was utilized to fabricate the sandwich specimens. During the VARTM process, the sandwich specimens were infused under the same conditions, i.e. temperature, humidity, and vacuum pressure $(760 \mathrm{mmHg}(1 \mathrm{~atm}))$, with the same volume of resin. The overall dimensions for the samples were approximately $102 \mathrm{~mm}$ wide, $254 \mathrm{~mm}$ long and 48 $\mathrm{mm}$ thick. The foam core itself was approximately $38 \mathrm{~mm}$ thick, while the skin thickness was approximately $5 \mathrm{~mm}$.
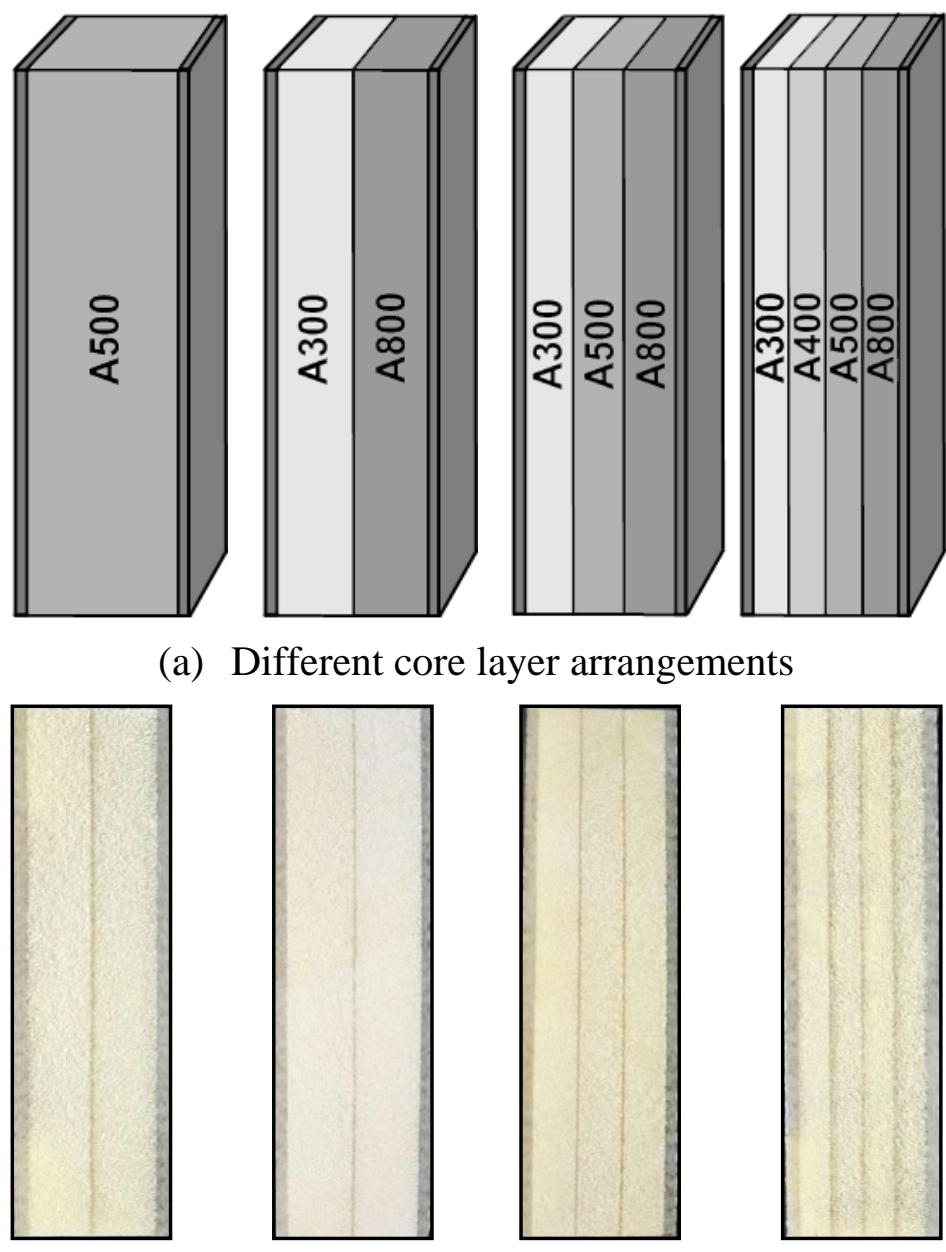

(a) Different core layer arrangements
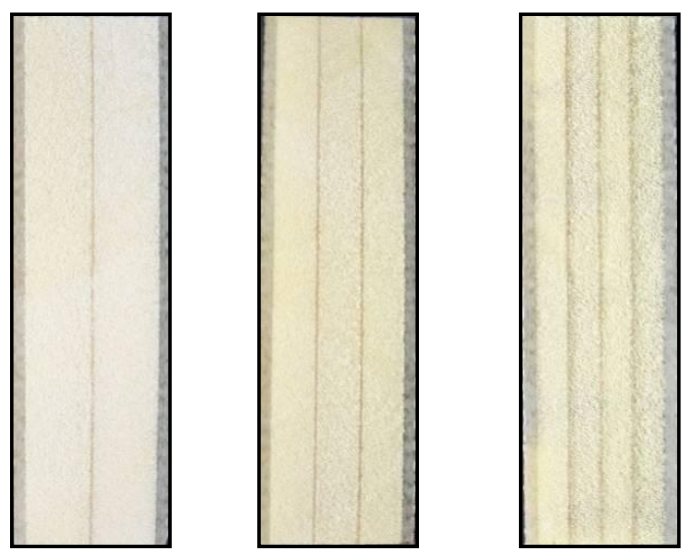

(b) Real specimens

Fig. 3 Specimen configuration and core gradation 
For the sandwich composites with a functionally graded/layered core, a series of four different core layer arrangements were studied (as shown in Fig. 3a). The four different core layer arrangements/gradations, along with their respected foam thickness and overall average areal densities are shown in Table 2. These core layer arrangements were functionally graded by monotonically increasing the onedimensional acoustic wave impedance.

Table 2. Foam core gradation and thickness

\begin{tabular}{ccccc}
\hline $\begin{array}{c}\text { Core } \\
\text { Gradation }\end{array}$ & $\begin{array}{c}\text { Core Layer } \\
\text { Arrangement }\end{array}$ & $\begin{array}{c}\text { Thickness of } \\
\text { Each Foam } \\
\text { Core Layer } \\
(\mathrm{mm})\end{array}$ & $\begin{array}{c}\text { Total } \\
\text { Thickness of } \\
\text { the Core } \\
(\mathrm{mm})\end{array}$ & $\begin{array}{c}\text { Average } \\
\text { Areal } \\
\text { Density } \\
\left(\mathrm{kg} / \mathrm{m}^{2}\right)\end{array}$ \\
\hline 1 Layer & A500/A500 & 19.05 & 38 & 19.0 \\
2 Layer & A300/A800 & 19.05 & 38 & 18.5 \\
3 Layer & A300/A500/A800 & 12.70 & 38 & 19.0 \\
4 Layer & A300/A400/A500/A800 & 9.53 & 38 & 20.0 \\
\hline
\end{tabular}

For one layer of core gradation, sandwich composites were created using A500 foam alone, and the core layer arrangement was A500/A500 (middle / middle density). For two layers of core gradation, sandwich composites were fabricated using A300 and A800 foams, and the core layer gradation was A300/A800 (lowest / high density). For three layers of core gradation, sandwich composites were created using A300, A500 and A800 foams, and the core layer gradation was A300/A500/A800 (lowest / middle / high density). Finally, for four layers of core gradation, sandwich composites were fabricated using A300, A400, A500, and A800 foams, and the core layer gradation was A300/A400/A500/A800 (lowest / low / middle / high density). With 
these configurations it should be noted that the first core layer is the one first subjected to the shock wave loading. Actual samples can be seen in Fig. $3 b$.

\section{Experimental Set-up and Procedure}

\subsection{Modified Split Hopkinson Pressure Bar with Hollow Transmission Bar}

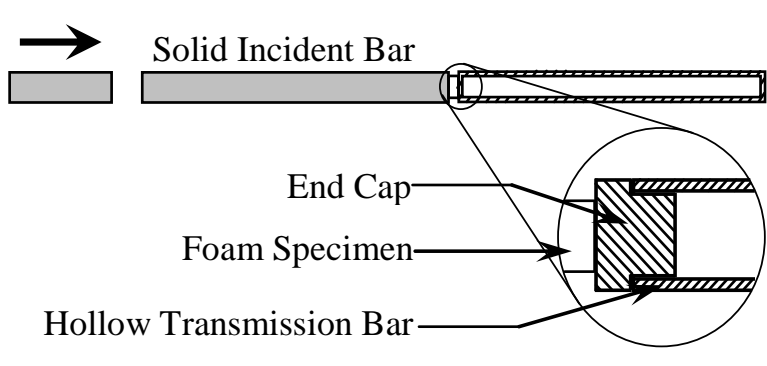

(a) Modified SHPB device

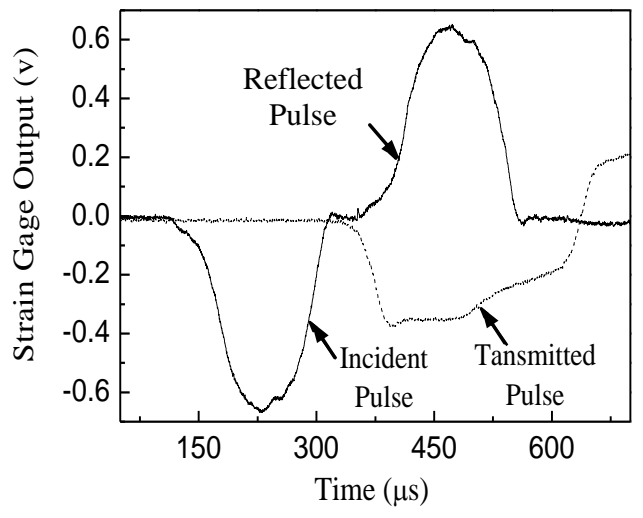

(b) Typical pulse profiles

Fig. 4 Sketch of modified SHPB device with hollow transmission bar and typical pulse profiles

A Split Hopkinson Pressure Bar (SHPB) is the most common device for measuring dynamic constitutive properties of materials. Due to the low-impedance of Corecell $^{\mathrm{TM}}$ foam materials, dynamic experiments for the core materials were performed with a modified SHPB device with a hollow transmission bar to increase the transmitted signal intensity. A sketch of the modified SHPB device and typical pulse profiles are given in Fig. 4. It has a $305 \mathrm{~mm}$-long striker, $1600 \mathrm{~mm}$-long incident bar and 1447 mm-long transmission bar. All of the bars are made of a 6061 aluminum alloy. The nominal outer diameters of the solid incident bar and hollow transmission bar are both $19.05 \mathrm{~mm}$. The hollow transmission bar has a $16.51 \mathrm{~mm}$ inner diameter. At the head and at the end of the hollow transmission bar, end caps made of the same material as the bar were press fitted into the hollow tube. By applying pulse shapers, the effect of the end caps on the stress waves can be minimized. The details of the 
analysis and derivation of equations for analysis of experimental data can be found in ref [20].

\subsection{Shock Tube}

The shock tube apparatus was utilized to obtain the controlled blast loading (Fig. 5a). It has an overall length of $8 \mathrm{~m}$, consisting of a driver, driven and muzzle section. The high-pressure driver section and the low-pressure driven section are separated by a diaphragm. By pressurizing the high-pressure section, a pressure difference across the diaphragm is created. When this difference reaches a critical value, the diaphragms rupture. This rapid release of gas creates a pressure wave that develops into a shock wave as it travels down the tube to impart dynamic loading on the specimen.

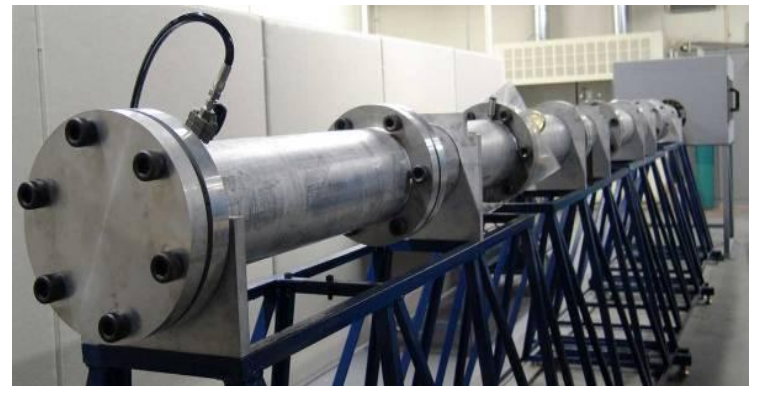

(a) Shock tube

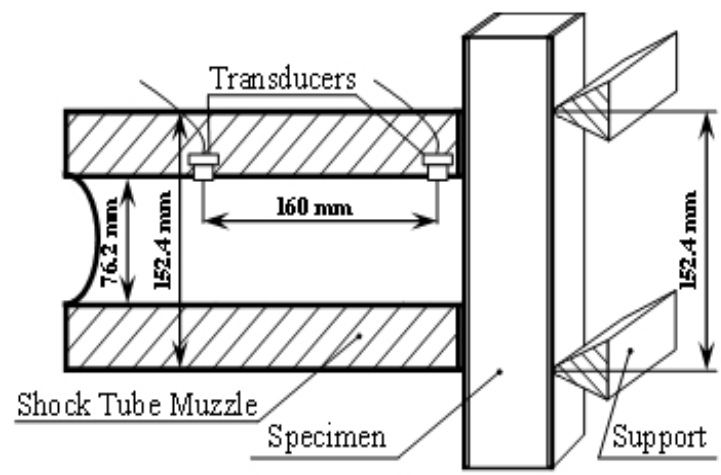

(b) Detailed dimensions of the muzzle

Fig. 5 Shock tube apparatus

Fig. $5 b$ shows detailed dimensions and locations of the muzzle, specimen, supports and the pressure sensors (PCB102A). The sensors are mounted at the end of the muzzle section to measure the incident pressure and the reflected pressure during the experiment. The final muzzle diameter is $0.076 \mathrm{~m}$. The distance between the two sensors is $0.160 \mathrm{~m}$ and the distance between the second sensor and the end of the muzzle is $\sim 0.020 \mathrm{~m}$. The specimen was placed in the supports and positioned close to 
the end of the muzzle. These support fixtures ensure simply supported boundary conditions with a $0.152 \mathrm{~m}$ span.

\subsection{High-speed Photography Systems}

Two high-speed photography systems were used in the present study, as shown in Fig. 6. A high-speed 3-D Digital Image Correlation (DIC) system, consisting of two high-speed digital cameras [Photron SA1], was placed facing the back side of the specimen to obtain the real-time full-field in-plane strain, along with out-of-plane deflection and velocity of the back facesheet. A speckle pattern was placed directly on the back facesheet of the sandwich composite to ensure good contrast of the images. Another high-speed digital camera, [Photron SA1], was placed perpendicular to the side surface of the specimen to capture the side-view deformation images. A framing rate of 20,000 fps was utilized which gives an interframe time of approximately $50 \mu$ s.

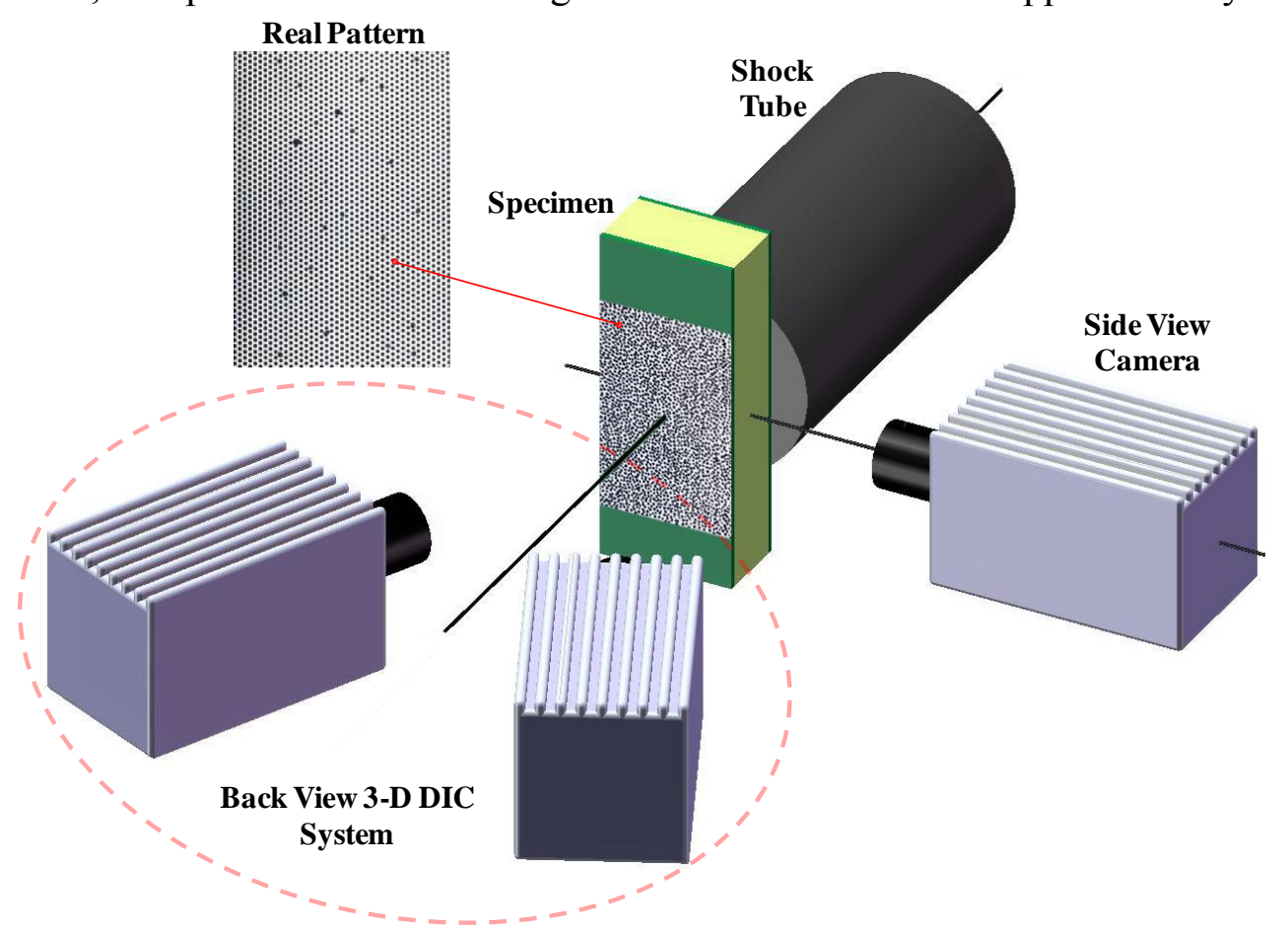

Fig. 6 High-speed photography set-up (Back-view DIC and side-view) 


\subsection{Experimental Procedure and Parameters}

In the present study, a simply stacked diaphragm of 5 plies of $0.254 \mathrm{~mm}$ mylar sheets with a total thickness of $1.270 \mathrm{~mm}$ was utilized to generate an impulse loading on the specimen with an incident peak pressure of approximately $1 \mathrm{MPa}$, a reflected peak pressure of approximately $5 \mathrm{MPa}$ and a shock wave speed of approximately 1000 $\mathrm{m} / \mathrm{s}$. For each configuration, at least three samples were tested. A typical pressure profile obtained from the transducer closest to the specimen ( $\sim 0.020 \mathrm{~m}$ away $)$ can be seen in Fig. 7. It should be noted that both pressure transducers were utilized to obtain the shock wave history, i.e. incident / reflected pressure and incident / reflected velocity. However, only the pressure transducer closest to the specimen was utilized to obtain the pressure applied on the specimen.

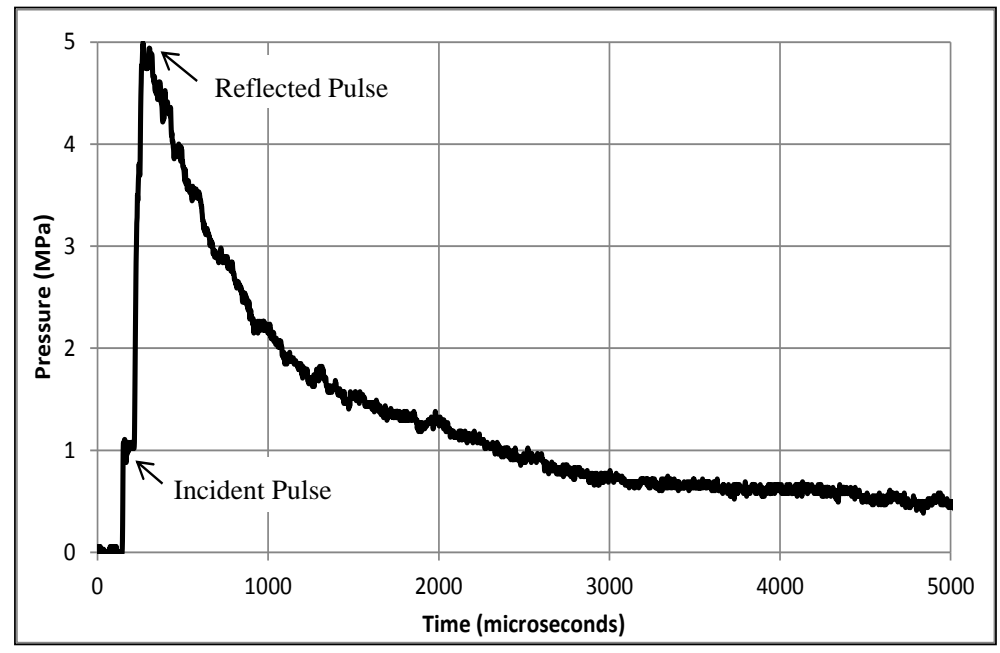

Fig. 7 Incident and reflected pressure profile 


\section{Experimental Results and Discussion}

\subsection{Dynamic Behavior of Core Material}

The four types of Corecell ${ }^{\mathrm{TM}} \mathrm{A}$-series foams have different quasi-static and dynamic compressive behavior. For the same type of Corecell ${ }^{\mathrm{TM}}$ foam, the material shows strain rate dependency from quasi-static to dynamic loading.

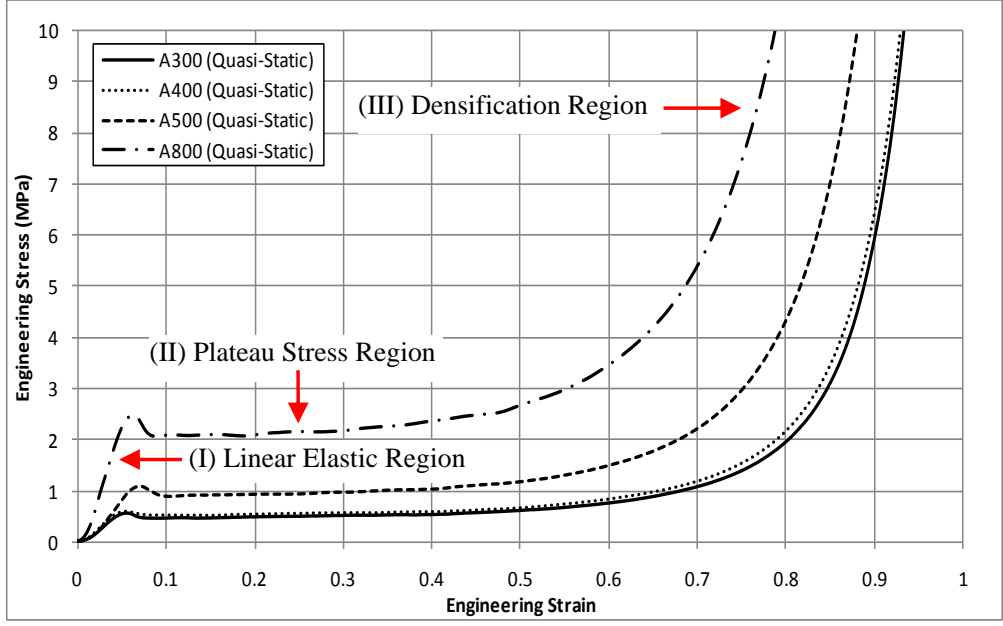

(a) Quasi-Static

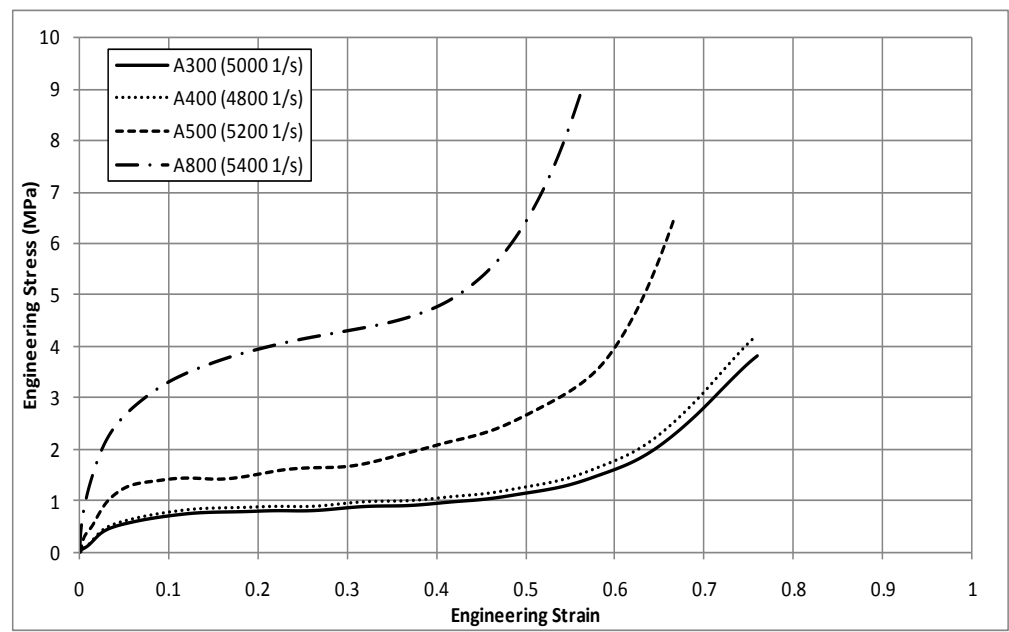

(b) Dynamic

Fig. 8 Compressive behavior of different types of Corecell ${ }^{\mathrm{TM}} \mathrm{A}$-series foams

Fig. 8 shows the quasi-static (Fig. 8a) and high strain rate (Fig. 8b) compressive behavior of the different types of Corecell ${ }^{\mathrm{TM}}$ A-series foams. For the quasi-static compressive behavior (Fig. 8a), the stress-strain curves show three deformation 
regions; (I) the linear elastic region, (II) the plateau stress (plastic yielding) region and (III) the densification region [21]. For high strain rate compressive behavior, the stress-strain curves also show the three deformation regions, even though the densification region is much harder to achieve. Note the plateau stress regions for both instances have a large strain range.

As seen in Fig. 8, the quasi-static and dynamic stress-strain responses have an obvious trend for the different types of foams. Lower density foam has a lower strength and stiffness, as well as a larger strain range for the plateau stress.

The high strain rate yield stresses and plateau (flow) stresses are much higher than the quasi-static ones for the same type of foams. Table 3 shows the quasi-static and high strain rate plateau stresses (measured in the plateau stress region). The dynamic strengths of A500 and A800 foam increase approximately $100 \%$ in comparison to their quasi-static strengths, while the dynamic strengths of A300 and A400 foam increase approximately $50 \%$ in comparison to their quasi-static strengths. The high yield stresses and long stress plateaus indicate that these foams can withstand higher stresses and absorb larger amounts of energy. Therefore, they show great potential in being used as core materials in sandwich structures subjected to high intensity blast loading.

Table 3. Flow stresses (plateau) of Corecell ${ }^{\mathrm{TM}}$ A-series foams

\begin{tabular}{ccccc}
\hline Foam Type & $\mathbf{A 3 0 0}$ & $\mathbf{A 4 0 0}$ & $\mathbf{A 5 0 0}$ & $\mathbf{A 8 0 0}$ \\
\hline $\begin{array}{c}\text { Quasi-Static } \\
\text { (MPa) }\end{array}$ & 0.48 & 0.56 & 0.88 & 2.08 \\
$\begin{array}{c}\text { High Strain } \\
\text { Rate (MPa) }\end{array}$ & 0.80 & 0.90 & 1.60 & 4.00 \\
\hline
\end{tabular}




\subsection{Blast Response of Sandwich Composites with Functionally Graded Cores}

\subsubsection{Real-time Deformation}

The real-time observations of the transient behavior for all types of configurations subjected to shock wave loading are shown in Fig. 9. The shock wave (pressure wave) propagates from the right side of the image to the left side and some detailed deformation mechanisms are pointed out in the figures. It should be noted that the time scheme used to represent the images in each configuration is identical. Therefore, for each of the four configurations investigated, the images are correlated based on the same time per frame. This allows for a better comparison between the different configurations in regards to the failure mechanisms and extent of damage observed. Also, since each configuration is graded monotonically by increasing the acoustic wave impedance, the damage processes are identical. First, indentation failure (initiation of core compression) is observed; followed by core compression, core cracking, and then finally delamination, either between the skin and core or at the core layer interfaces.

Fig. 9a shows the real-time blast loading response for the sandwich composites with one layer of core gradation. From these images, it can be seen that the indentation failure begins at approximately $\mathrm{t}=100 \mu \mathrm{s}$. At $\mathrm{t}=400 \mu$ s core cracking is evident. The crack starts at the lower support and propagates from the back face towards the front face. By $\mathrm{t}=700 \mu \mathrm{s}$ more core cracking is observed and delamination between the core layers can be seen. Skin delamination is evident at $t=1000 \mu$ s between the front facesheet and first core layer of foam, along with heavy first layer core compression. By $\mathrm{t}=1600 \mu$ s the core cracks have propagated completely through the core from the 
back face to the front face, and the amount of delamination has increased. Also, the first layer core compression has reached a maximum, approximately $30 \%$ of its original thickness.

(a)

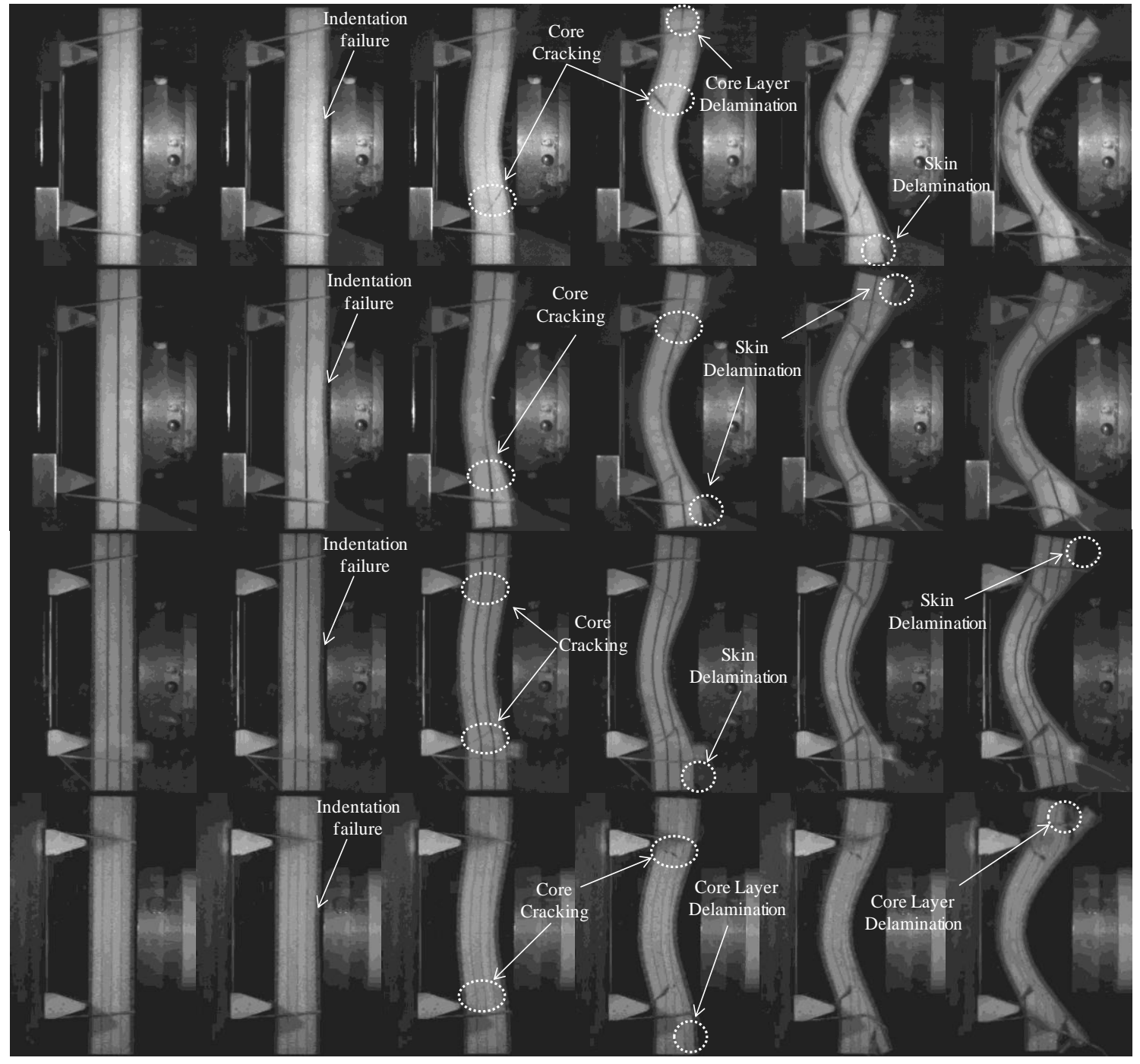

$\mathrm{t}=0 \mu \mathrm{s}$

$\mathrm{t}=100 \mu \mathrm{s}$

$\mathrm{t}=400 \mu \mathrm{s}$

$\mathrm{t}=700 \mu \mathrm{s}$

$\mathrm{t}=1000 \mu \mathrm{s}$

$\mathrm{t}=1600 \mu \mathrm{s}$

Fig. 9 High-speed images for (a) One layer (b) Two layer (c) Three layer and (d) Four layer of core gradation

The real-time blast loading response for the sandwich composites with two layers of core gradation can be seen in Fig. 9b. Indentation failure for this configuration is observed at approximately $\mathrm{t}=100 \mu \mathrm{s}$. By $\mathrm{t}=400 \mu \mathrm{s}$, first layer core compression is 
very evident (A300). Also at this time, core cracking starting from the back facesheet where the lower support is located can be seen. By $t=700 \mu$ s more core cracking can be observed, as well as skin delamination between the front skin and the first core layer of foam (located at the bottom of the specimen). At $t=1000 \mu$ s more skin delamination can be observed between the front facesheet and the first core layer of foam, but this time it is located at the top of the specimen. By $\mathrm{t}=1600 \mu \mathrm{s}$, the core cracks have propagated completely through the core from the back facesheet to the front facesheet. Also, the core compression in the first core layer has reached a maximum, approximately $75 \%$ of its original layer thickness.

Fig. 9c shows the real-time blast loading response for the sandwich composites with three layers of core gradation. It can be seen from these images that the indentation failure begins at approximately $\mathrm{t}=100 \mu \mathrm{s}$. Following indentation failure, heavy first layer (A300) core compression is observed as well as core cracking by approximately $\mathrm{t}=400 \mu \mathrm{s}$. By $\mathrm{t}=700 \mu \mathrm{s}$, the first core layer has compressed to a maximum, reaching a critical strain level and initiating the onset of indentation and compression in the second core layer of foam (A500). Also at this time, skin delamination between the front skin and the first core layer of foam is evident at the bottom of the specimen. At $t=1000 \mu$ s, more core compression is observed in the second core layer. Finally by $\mathrm{t}=1600 \mu$ s, skin delamination can be seen at the top of the specimen between the front facesheet and the first core layer of foam. The core cracking has propagated completely through the core from the back facesheet to the front facesheet. Also at this time, the core compression in the first and second layers has reached a maximum, approximately $70 \%$ and $50 \%$ respectively. 
The real-time blast loading response for the sandwich composites with four layers of core gradation are shown in Fig. 9d. From these images, indentation failure is observed at approximately $\mathrm{t}=100 \mu \mathrm{s}$. At $\mathrm{t}=400 \mu \mathrm{s}$, heavy core compression is observed in the first core layer (A300). Also at this time, core cracking has initiated and is located at the bottom of the specimen where the supports are. By $\mathrm{t}=700 \mu \mathrm{s} \mathrm{a}$ second core crack is evident at the top of the specimen where the upper support is located. Delamination is evident at the bottom of the specimen, between the first and second core layers of foam. Also at this time, the first core layer of foam has compressed to a maximum, reaching a critical strain level and initiating the onset of indentation and compression in the second core layer of foam (A400). By $\mathrm{t}=1000 \mu \mathrm{s}$, the second core layer has compressed to a maximum, reaching a critical strain level, causing indentation and compression in the third core layer of foam (A500). Finally by $\mathrm{t}=1600 \mu \mathrm{s}$, the total core compression has reached a maximum, the first layer and second core layer have compressed approximately $65 \%$, while the third layer has compressed approximately $30 \%$. Also at this time, delamination between the first and second core layer of foam is evident, and occurs at the top of the specimen. The core cracks have stopped propagating towards the front facesheet, and unlike the other configurations studied, these core cracks never propagated completely through the core.

In order to better evaluate the blast performance of the four different core layer arrangements studied, a more in depth look at the later time frames $(\mathrm{t}=1000 \mu \mathrm{s}$ and onward) of the high-speed images (Fig. 9) must be investigated. For the sandwich composite with one layer of core gradation (as shown in Fig. 9a), it is evident at $t=$ 
$1000 \mu$ s there are approximately four (4) major damage areas present. These damage areas consist of two heavy core cracks occurring in the central region of the specimen, one where the bottom support is located and the other just below the top support, as well as core delamination at the top of the specimen between the foam core layers, and skin delamination at the bottom of the specimen between the front facesheet and first core layer of foam.

Using two layers of core gradation, as shown in Fig. 9b, at $t=1000 \mu$ s again four (4) major damage areas exist. These damage areas include two heavy core cracks in the central region of the specimen, where the supports are located, as well as skin delamination between the front facesheet and first core layer of foam, both at the bottom and top of the specimen.

Utilizing three layers of core gradation, as shown in Fig. 9c, at $\mathrm{t}=1000 \mu$ s only three (3) damage areas are present. These damage areas include two core cracks located in the central region of the specimen where the supports are located, as well as skin delamination, which occurs at the bottom of the specimen between the front facesheet and first layer of foam core. By $\mathrm{t}=1600 \mu \mathrm{s}$, the fourth damage area becomes present and consists of skin delamination between the front skin and foam core which occurred at the top of the specimen.

For the sandwich composite with four layers of core gradation, as shown in Fig. 9d, again it can be observed that at $\mathrm{t}=1000 \mu$ s only three (3) areas of damage are present. These damage areas consist of two core cracks occurring wear the supports are located and propagating into the central region of the specimen. Also at this time core delamination can be observed, which occurs at the bottom of the specimen 
between the first and second core layer of foam. The fourth damage zone can be observed at $\mathrm{t}=1600 \mu \mathrm{s}$. This damage area consists of core delamination at the top of the specimen, which occurs between the first and second core layer of foam.

Therefore, by looking at Fig. 9, it is clearly evident that when using sandwich composites with one and two layers of core gradation, four damage areas are present by $t=1000 \mu \mathrm{s}$. When using sandwich composites with three and four layers of core gradation, only three (3) damage areas are present at $t=1000 \mu$ s. The fourth damage zone isn't observed until later in the panel deformation. Therefore, it can be concluded that higher levels of core layer gradation (i.e. 3 and 4 layers), allow for a delay in the arrival of the fourth damage zone.

\subsubsection{Deflection}

The mid-point deflections of each graded sandwich panel and all of its constituents were obtained from the high-speed images and a typical response can be seen in Fig. 10. For one layer gradation, the midpoint deflection for the front face (front skin) and back face (back skin) of the specimen was plotted and can be seen in Fig. 10a. It is evident from the figure that at $t=1600 \mu$ s the front face deflects to approximately $46 \mathrm{~mm}$, while the back facesheet deflects approximately $35 \mathrm{~mm}$. Therefore, the difference between the deflection of the front face and deflection of the back face signifies the total amount of compression observed in the core. Therefore, it can be concluded that the core compressed approximately $11 \mathrm{~mm}$, which is $30 \%$ of its original thickness $(38 \mathrm{~mm})$. 


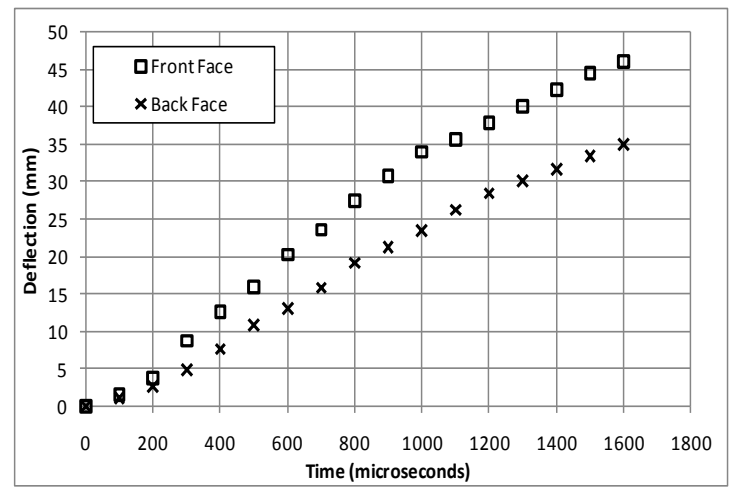

(a) One Layer Gradation

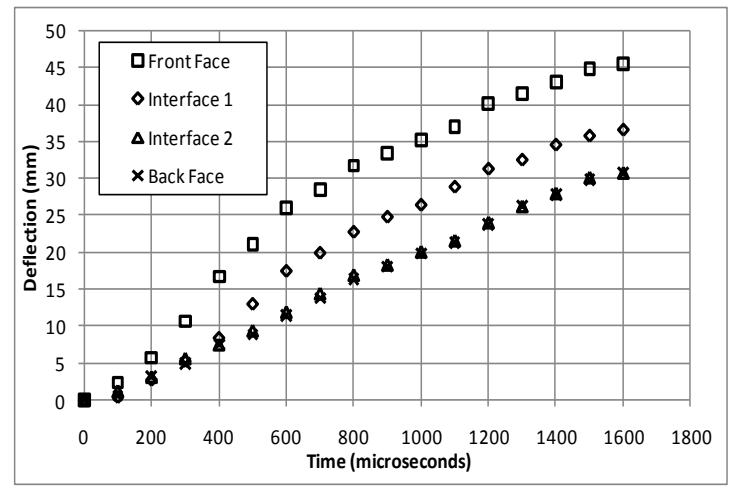

(c) Three Layer Gradation

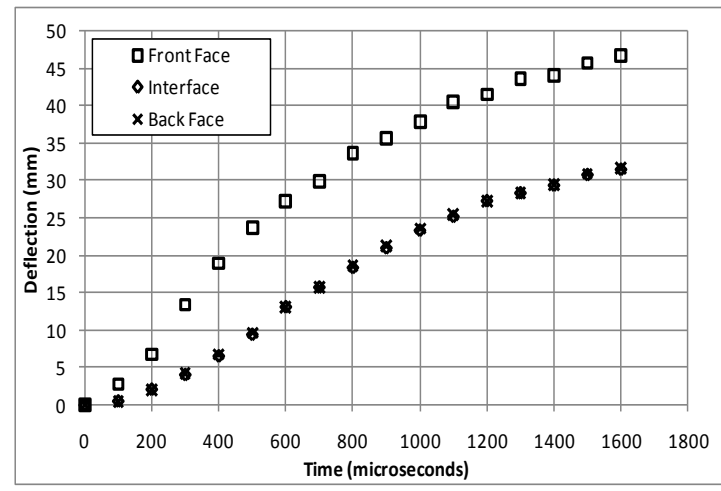

(b) Two Layer Gradation

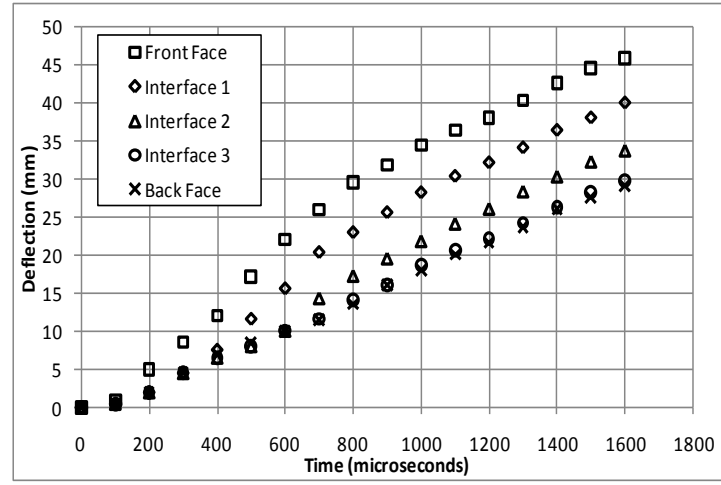

(d) Four Layer Gradation

Fig. 10 Mid-point deflection of all configurations

When using two layers of core gradation, the midpoint deflection of the front face (front skin), interface (between first and second core layer), and back face of the specimen were plotted and are shown in Fig. 10b. It can be seen from the figure that at $\mathrm{t}=1600 \mu \mathrm{s}$, the front face has deflected to approximately $46 \mathrm{~mm}$, while the interface and the back face deflect to approximately $33 \mathrm{~mm}$. The difference between the deflection of the front face and deflection of the interface indicates the total amount of compression observed in the first core layer of foam (A300 layer). Therefore, the first core layer of foam (A300) compressed approximately $13 \mathrm{~mm}$, or $75 \%$ of its original thickness $(19.05 \mathrm{~mm})$. Since the interface and the back face deflect in an identical 
manner to the same value of $33 \mathrm{~mm}$, it can be concluded that no compression was observed in the second core layer of foam (A800).

For three layers of core gradation, the midpoint deflection for the front face (front skin), interface 1 (between first and second core layer), interface 2 (between second and third core layer), and back face (back skin) were plotted and can be seen in Fig.10c. It is evident from the figure that at $\mathrm{t}=1600 \mu$ s, the front face has deflected to approximately $46 \mathrm{~mm}$, while interface 1 deflects to approximately $37 \mathrm{~mm}$, and interface 2 as well as the back face deflect to approximately $31 \mathrm{~mm}$. The difference between the deflection of the front face and the deflection of interface 1 constitutes the total amount of compression in the first core layer of foam (A300). Therefore, it can be seen that A300 foam compressed approximately $9 \mathrm{~mm}$, which is $~ 75 \%$ of its original thickness $(12.70 \mathrm{~mm})$. Looking at the difference between the deflection of interface 1 and the deflection of interface 2, the amount of compression observed in the second core layer of foam (A500) can be obtained. It can be seen that the A500 foam compressed approximately $6 \mathrm{~mm}$, or $\sim 50 \%$ of its original thickness $(12.70 \mathrm{~mm})$. Again, since interface 2 and the back face deflect in an identical manner to the same value of $31 \mathrm{~mm}$, it can be concluded that no compression was observed in the third core layer of foam (A800).

Finally, when using four layers of core gradation, the midpoint deflection of the front face (front skin), interface 1 (between first and second core layer), interface 2 (between second and third core layer), interface 3 (between third and fourth core layer), and back face (back skin) were plotted and are shown in Fig. 10d. It can be seen from the figure that at $\mathrm{t}=1600 \mu \mathrm{s}$, the front face has deflected to approximately 
$46 \mathrm{~mm}$, while interface 1 has deflected to approximately $39 \mathrm{~mm}$, interface 2 has deflected to approximately $33 \mathrm{~mm}$, while interface 3 and the back facesheet deflect to approximately $29 \mathrm{~mm}$. The difference between the deflection of the front face and the deflection of interface 1 signifies the total amount of compression in the first core layer of foam (A300). Therefore, it can be seen that A300 foam compresses approximately $7 \mathrm{~mm}$, which is $\sim 70 \%$ of its original thickness $(9.53 \mathrm{~mm})$. Looking at the difference between the deflection of interface 1 and the deflection of interface 2 , the amount of compression observed in the second core layer of foam (A400) can be obtained. It can be seen that the A400 foam compresses approximately $6 \mathrm{~mm}$, or $65 \%$ of its original thickness $(9.53 \mathrm{~mm})$. The difference between the deflection of interface 2 and the deflection of interface 3 indicates the total amount of compression observed in the third layer of foam (A500). Therefore, it is evident that the A500 foam compressed approximately $4 \mathrm{~mm}$, which is $\sim 40 \%$ of its original thickness $(9.53 \mathrm{~mm})$. Since interface 3 and the back face deflect in an identical manner to the same value of $29 \mathrm{~mm}$, it can be concluded that no compression was observed in the fourth core layer of foam (A800).

It should be noted that for all of the configurations studied, the core layers were graded monotonically by increasing the acoustic wave impedance, and therefore allowing for a stepwise compression of the core. This stepwise compression is more evident in the three and four layer core configurations, i.e. Fig. 10c and Fig. 10d.

From the deflection data of each interface in Fig. 10, the deformation of each core layer along the mid- line (line of symmetry) can be obtained by subtracting the core layers' back side deflection from the core layers' front side deflection. Sequentially, 
the strain along the line of symmetry of each core layer can be obtained using the following equation,

$$
\text { strain }=\varepsilon=\frac{(\Delta \mathrm{l})}{1_{\text {original }}}
$$

where, $l_{\text {original }}$ is the original thickness of the each core layer.

The strain histories of the core layers for each configuration, as calculated from Eq. (3) using the mid-point deflection data from Fig. 10, are shown in Fig. 11 respectively. Since there is no compression in the A800 foam core layer, its strain history is not shown here.

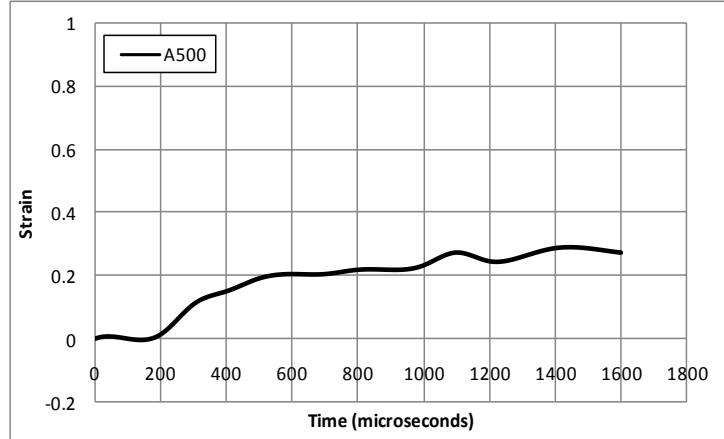

(a) One Layer Gradation

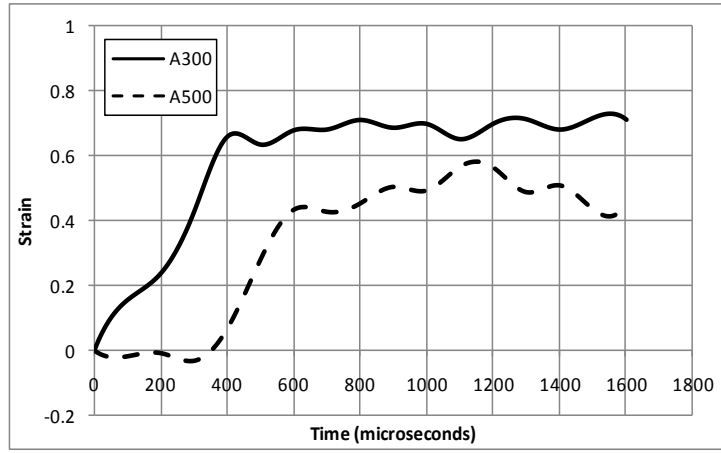

(c) Three Layer Gradation

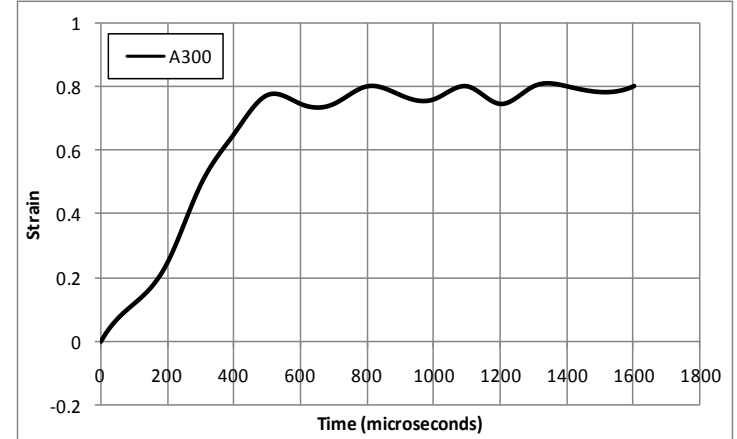

(b) Two Layer Gradation

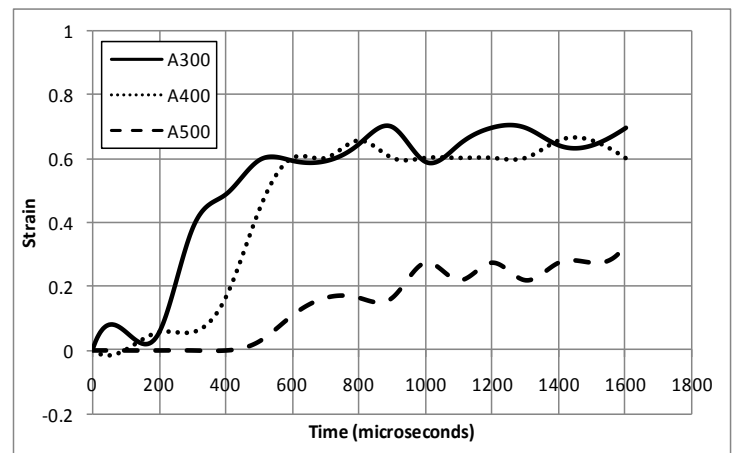

(d) Four Layer Gradation

Fig. 11 Strain history of each core layer along the mid-line (line of symmetry) for all core configurations

It is evident from the figure when using sandwich composites with higher levels of core gradation, the maximum strain levels achieved in the same individual core layer of foam is reduced. For the A300 foam core layer, the maximum strain level 
achieved using two layers of core gradation (Fig. 11b) is approximately $75 \%-80 \%$. When using higher levels of core gradation, three and four layers (Fig.11c and Fig. 11d), the A300 layer exhibits a maximum strain level of approximately $70 \%$ and $65 \%$ respectively. For the A500 foam core layer, the maximum strain level achieved using three layers of core gradation (Fig. 11c) was approximately 50\%, while the strain level was reduced to approximately $30 \%$ when using four layers of core gradation (Fig. 11d). Note that even though the A500 foam core layer exhibited its lowest maximum strain value when using one layer of core gradation (Fig. 11a), heavy core cracking was observed early in the deformation history and subsequently its strain values and overall behavior will not be discussed in full.

For the sandwich composite with two layers of core gradation (Fig. 11b), the A300 core layer exhibited a maximum strain level of approximately $75 \%-80 \%$ at $\mathrm{t}=$ $500 \mu$ s, which corresponds to a stress level of approximately 3.8 MPa (Fig. 8b). Note that the stress level required to initiate compression (increase strain) in the second core layer (A800) is approximately 4.0 MPa (Fig. 8b). Therefore, no compression (strain) is observed in the A800 foam layer.

For the sandwich composite with three layers of core gradation (Fig. 11c), the maximum strain level achieved in the A300 foam was approximately $70 \%$ at $\mathrm{t}=400$ $\mu \mathrm{s}$, which corresponds to a stress level of $2.8 \mathrm{MPa}$ (Fig. $8 \mathrm{~b}$ ). Note that the stress level required to initiate compression (increase strain) in the second core layer (A500) is approximately 1.6 MPa (Fig. 8b). Therefore, core compression (strain) is expected in this layer of foam by $t=400 \mu \mathrm{s}$. In regards to the A500 layer, this layer exhibited a maximum strain level of approximately $50 \%$ at $\mathrm{t}=900 \mu \mathrm{s}$, which corresponds to a 
stress level of approximately $2.6 \mathrm{MPa}$ (Fig. 8b). Note that the stress level required to initiate compression (increase strain) in the third core layer (A800) is approximately 4.0 MPa (Fig. 8b). Therefore, no compression (strain) is observed in the A800 foam layer.

For the sandwich composite with four layers of core gradation (Fig. 11d), the maximum strain level achieved in the A300 foam was approximately $60 \%-65 \%$ by $\mathrm{t}$ $=600 \mu \mathrm{s}$, which corresponds to a stress level of approximately $2 \mathrm{MPa}$ (Fig. 8b). Note that the stress level required to initiate compression (increase strain) of the second core layer (A400) is approximately 0.9 MPa (Fig. 8b). Therefore, core compression (strain) is expected in this layer of foam by $t=600 \mu \mathrm{s}$. For the A400 foam core layer, this layer exhibited a maximum strain level of approximately $60-65 \%$ by $\mathrm{t}=600 \mu \mathrm{s}$, which corresponds to a stress level of approximately $2.1 \mathrm{MPa}$ (Fig. 8b). Note that the stress level required to initiate compression (increase strain) of the third core layer (A500) is approximately 1.6 MPa (Fig. 8b). Therefore, core compression (strain) is expected in this layer of foam by $\mathrm{t}=600 \mu \mathrm{s}$. For the A500 foam core layer, this layer exhibited a maximum strain level of approximately $30 \%$, which corresponds to a stress level of approximately $1.8 \mathrm{MPa}$ (Fig. 8b). Note that the stress level required to initiate compression (increase strain) of the fourth core layer (A800) is approximately 4.0 MPa (Fig. 8b). Therefore, no compression (strain) will be observed in the A800 foam layer.

Table 4 shows the exact strain (dynamic) values necessary to initiate compression in each of the subsequent foam core layers, as generated from Fig. 8b. Correlating these strain values, to the strain-time history plot in Fig. 11, the exact time of 
compression in each of the successive foam core layers for all gradations can be generated.

Table 4. Dynamic strain required to initiate compression in subsequent foam core layers

\begin{tabular}{ccccc}
\hline $\begin{array}{c}\text { Foam } \\
\text { Type }\end{array}$ & $\mathbf{A 3 0 0}$ & $\mathbf{A 4 0 0}$ & $\mathbf{A 5 0 0}$ & $\mathbf{A 8 0 0}$ \\
\hline $\mathbf{A 3 0 0}$ & - & $30 \%(300 \mu \mathrm{s})$ & $60 \%(400-500 \mu \mathrm{s})$ & $80 \%$ \\
$\mathbf{A 4 0 0}$ & - & - & $55 \%(500-600 \mu \mathrm{s})$ & $75 \%$ \\
$\mathbf{A 5 0 0}$ & - & - & - & $60 \%$ \\
$\mathbf{A 8 0 0}$ & - & - & - & - \\
\hline
\end{tabular}

An interesting phenomenon can be observed from deflection data and strain results in Fig. 10 and Fig. 11 respectively. By looking at the deflection data (Fig. 10) for two, three and four layers of core gradation by $t=\sim 600 \mu$ s, the foam core of each configuration has compressed the same amount, approximately $13 \mathrm{~mm}$. Using the same time, $\mathrm{t}=\sim 600 \mu \mathrm{s}$, and referring to the strain history of each configuration (Fig 11), for a sandwich composite with two layers of core gradation, only the first foam core layer (A300) is compressed. For higher levels of core gradation, i.e. three and four layers, by $\mathrm{t}=600 \mu \mathrm{s}$, compression in multiple layers has initiated, and thus increasing strain values in subsequent layers can be observed. In regards to the sandwich composite with three layers of core gradation, both the first (A300) and second (A500) core layers exhibit increasing levels of strain (compression). When using four layers of core gradation, the first (A300), second (A400) and third (A500) foam core layer all show increasing levels of strain (compression). Even though the total amount of core compression is the same $(\sim 13 \mathrm{~mm})$ in all core configurations, the 
compression of the denser foams (A400 and A500) in the panels with higher levels of gradation (three and four layers), signifies their ability to absorb more energy.

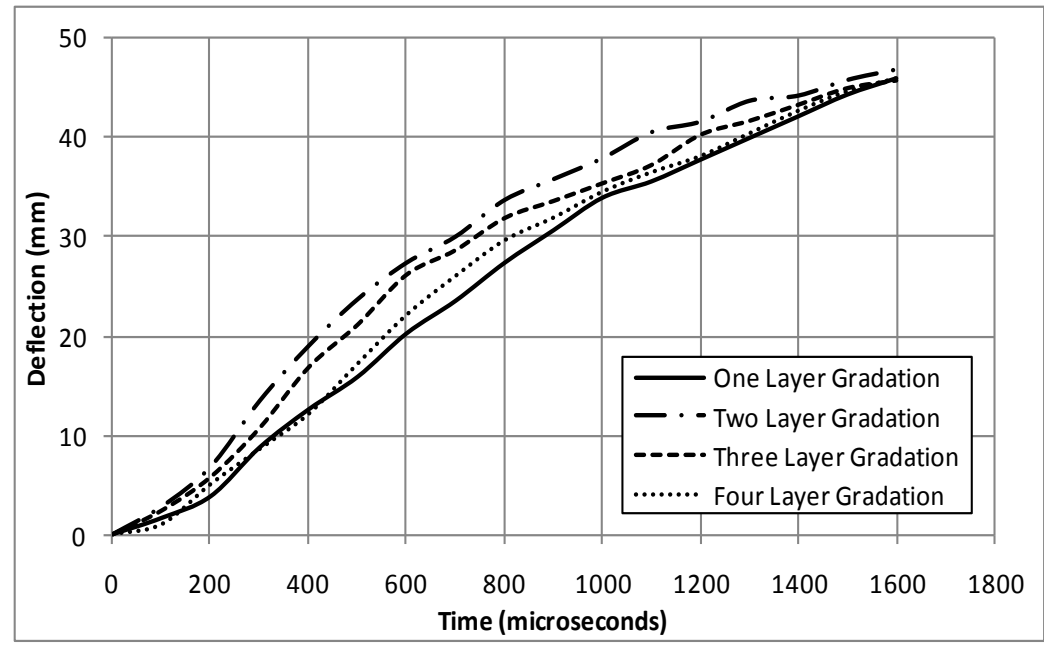

(a) Front face deflection

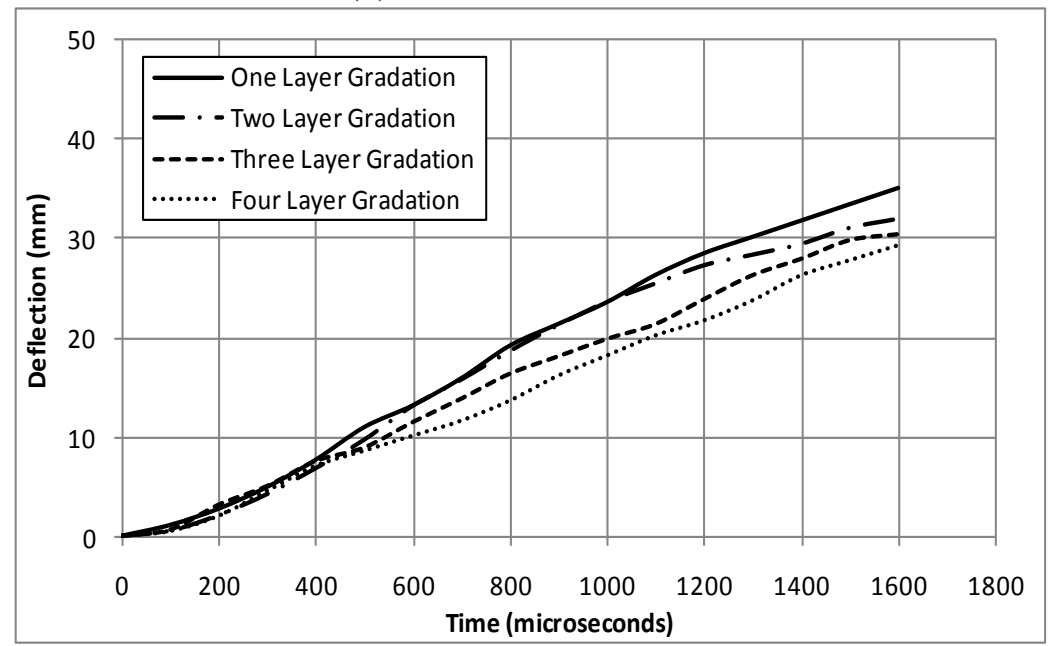

(b) Back face deflection

Fig. 12 Front and back face deflection comparison of all core configurations

Fig. 12 shows a comparison between the front face deflection (a) and the back face deflection (b) for the four different core configurations. It is evident from Fig. 12a, that at $\mathrm{t}=1600 \mu \mathrm{s}$, the front face deflection for all core configurations are in excellent agreement (within 5\%) It can also be observed that two layers of core gradation showed the most front face deflection, followed by three layers of core 
gradation. This is due to the thickness of the A300 foam layer (lowest density) located behind the front facesheet and its ability to compress. For two layer gradation and three layer gradation, the A300 layer of foam is approximately $19.05 \mathrm{~mm}$ and 12.70 mm respectively.

When looking at Fig. 12b, an interesting phenomenon is observed. At $\mathrm{t}=1600 \mu \mathrm{s}$, the back face deflection for the sandwich composite with one layer of core gradation is approximately $35 \mathrm{~mm}$, which is followed by two layer gradation, three layer gradation, and four layer gradation, with back face deflections of approximately $33 \mathrm{~mm}, 31 \mathrm{~mm}$ and $29 \mathrm{~mm}$ respectively. Therefore, in relation to the back face deflection of the sandwich composite with one layer gradation, two layer gradation deflects $\sim 6 \%$ less, three layer gradation deflects $\sim 11 \%$ less, and four layer gradation deflects $\sim 17 \%$ less.

\subsubsection{Digital Image Correlation (DIC) Analysis}

Utilizing the Digital Image Correlation (DIC) technique, the full-field deflection, in-plane strain and particle velocity of the back facesheet of each configuration was generated. Figures 13, 14, 15 show the full-field results for the back facesheet of all core gradations respectively. Fig. 13 shows the full-field out-of-plane deflection (W) during the initial fluid-structure interaction ( $\mathrm{t} \leq \sim 250 \mu \mathrm{s}$, [22]), with an emphasis on the shape of the loading, as indicated by the localized areas of larger deflection. Note that the scale used to represent each core gradation is different in order to highlight these areas. For one layer core gradation, as shown in Fig. 13a, by $\sim \mathrm{t}=150 \mu \mathrm{s}$, the loading can be observed as a circular region in the center of the back facesheet. For two, three and four layer core gradation, as shown in Figures 13b, 13c, and 13d respectively, by $t=150 \mu$ s the loading is more dispersed across the back facesheet, 
resulting in two and even three areas of localized deflection (loading). Therefore, it can be concluded that utilizing multiple layers of core gradation, and thus introducing more material interfaces, aids in dispersing the initial loading on the structure, resulting in up to three areas of localized deflection (loading) on the back facesheets.

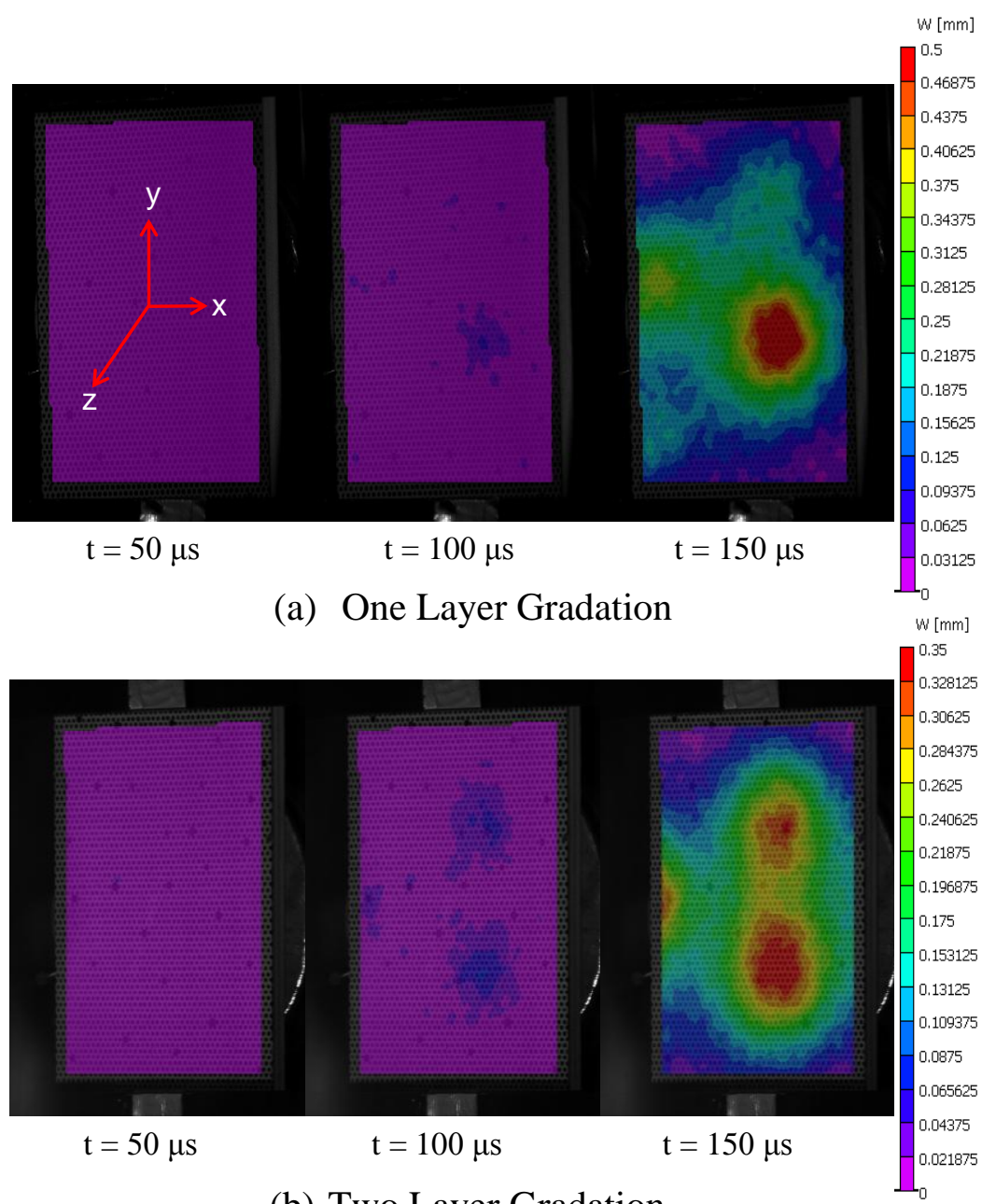

(b) Two Layer Gradation 


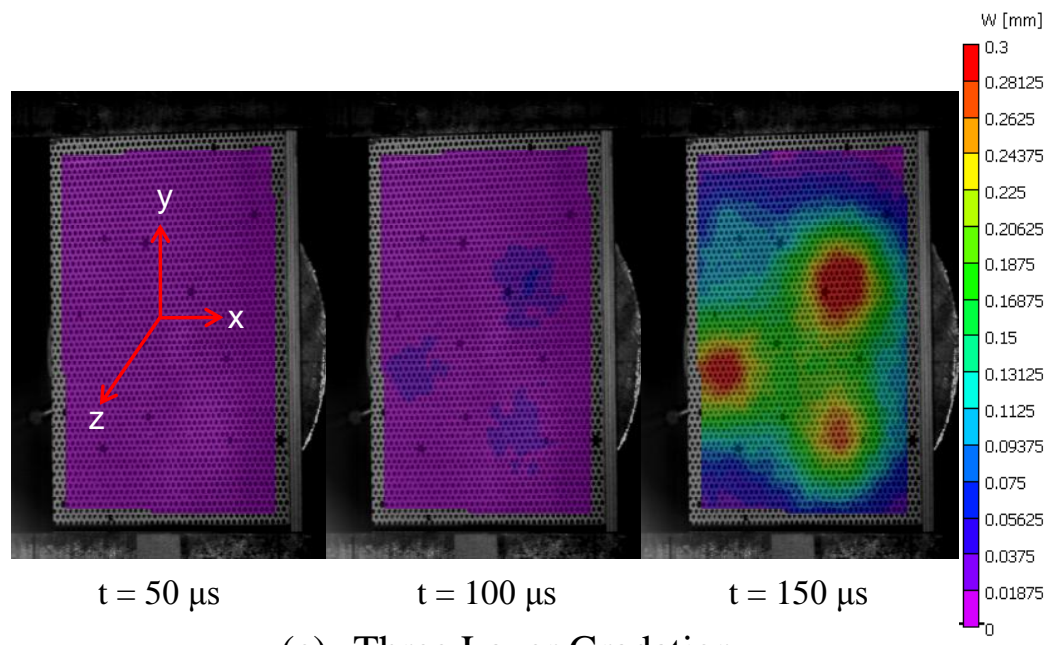

(c) Three Layer Gradation

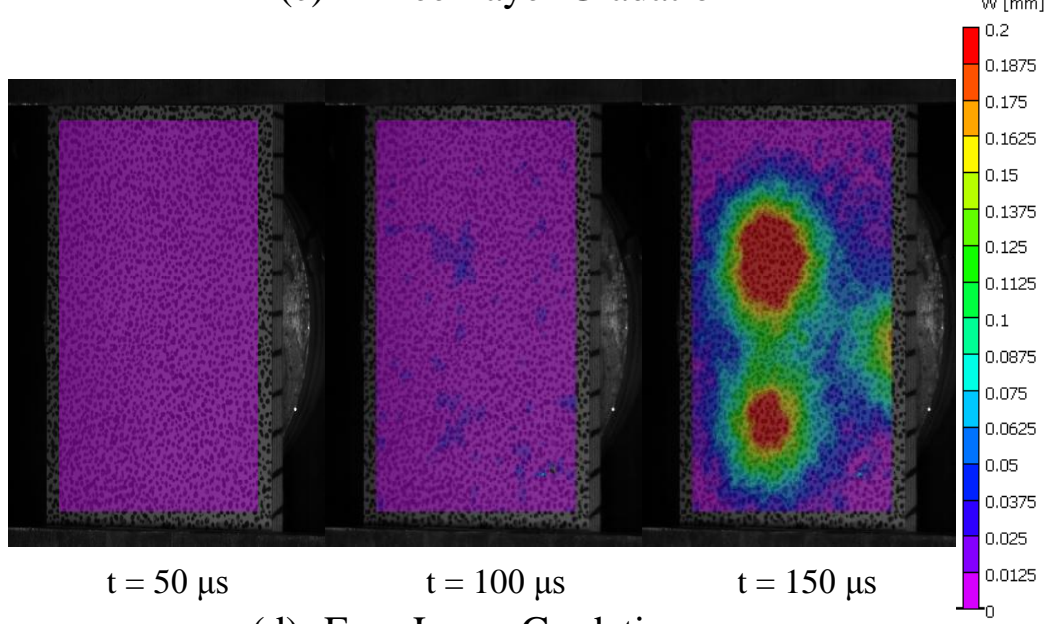

(d) Four Layer Gradation

Fig. 13 Localized areas of larger deflections (loading) during fluid-structure

Fig. 14 shows the full-field out-of-plane deflection (W) for all core layer gradations. Note the scale ranges from $0 \mathrm{~mm}$ (purple) to $40 \mathrm{~mm}$ (red). It is evident from the figure that the back face of all core configurations exhibits limited out-ofplane deflection prior to $\mathrm{t}=400 \mu \mathrm{s}$. Between $\mathrm{t}=400 \mu \mathrm{s}$ and $\mathrm{t}=1600 \mu \mathrm{s}$, all four of these configurations continue to bend and show deflections. For the sandwich composite with one layer of core gradation, as shown in Fig. 14a, it can be observed that at $\mathrm{t}=1600 \mu \mathrm{s}$, the central region of the panel has deflected approximately $36 \mathrm{~mm}$. When using two layers of core gradation (Fig. 14b), it can be seen that at $\mathrm{t}=1600 \mu \mathrm{s}$, 
the central region of the panel has deflected approximately $34 \mathrm{~mm}$. For the sandwich composite with three layers of core gradation, Fig. 14c, at $t=1600 \mu \mathrm{s}$, the central region has deflected approximately $32 \mathrm{~mm}$. Finally for the sandwich composite with four layers of core gradation (Fig. 14d), the central region of the panel has deflected $30 \mathrm{~mm}$ at $\mathrm{t}=1600 \mu \mathrm{s}$. Therefore, it can be concluded that utilizing more layers of core layer gradation, i.e. two, three and four layers, the deflection in the central region of the sandwich panel can be reduced $5 \%, 11 \%$ and $17 \%$ respectively, in comparison to one layer of core gradation.

(a)

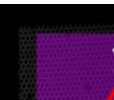

\section{y}

(b)

(c)

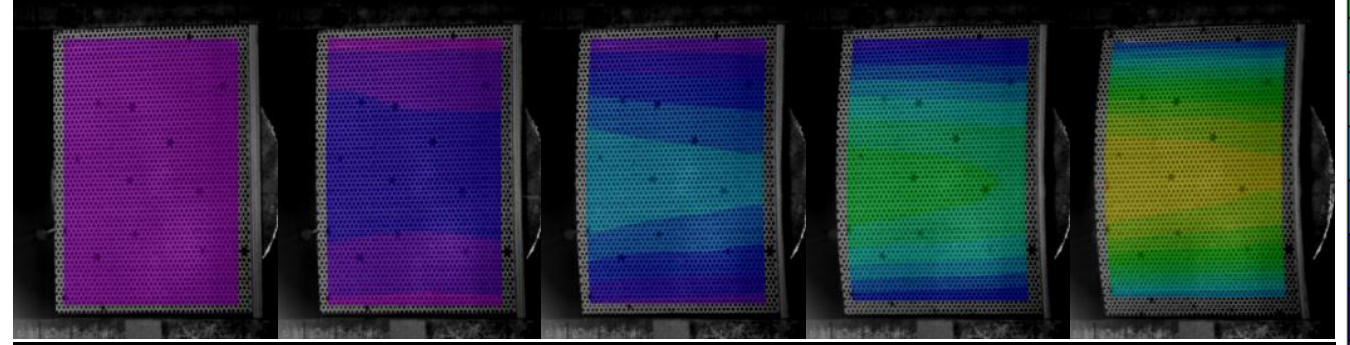

(d)

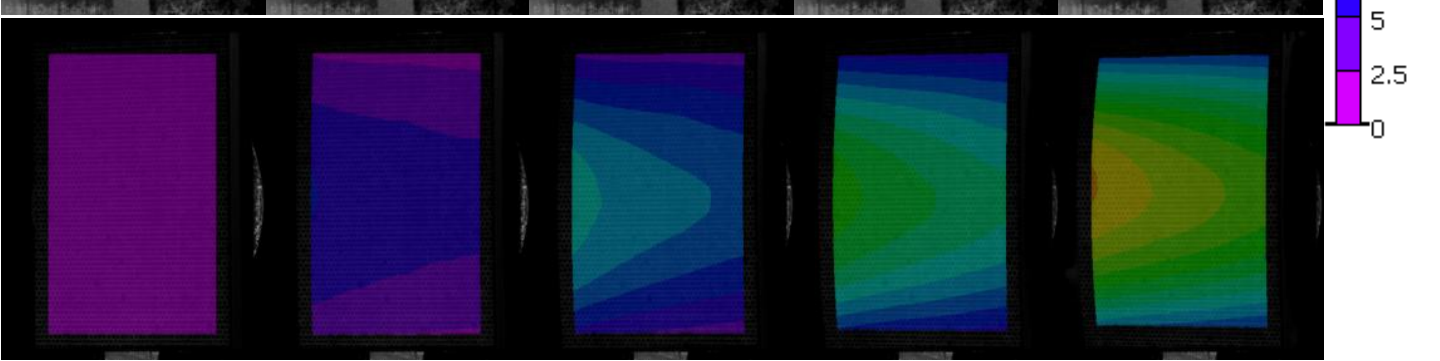

$\mathrm{t}=100 \mu \mathrm{s}$

$\mathrm{t}=400 \mu \mathrm{s}$

$\mathrm{t}=700 \mu \mathrm{s}$

$\mathrm{t}=1000 \mu \mathrm{s}$

$\mathrm{t}=1600 \mu \mathrm{s}$

Fig. 14 Full-field out-of-plane deflection (W) for (a) One layer (b) Two layers

(c) Three layers and (d) Four layers of core gradation 


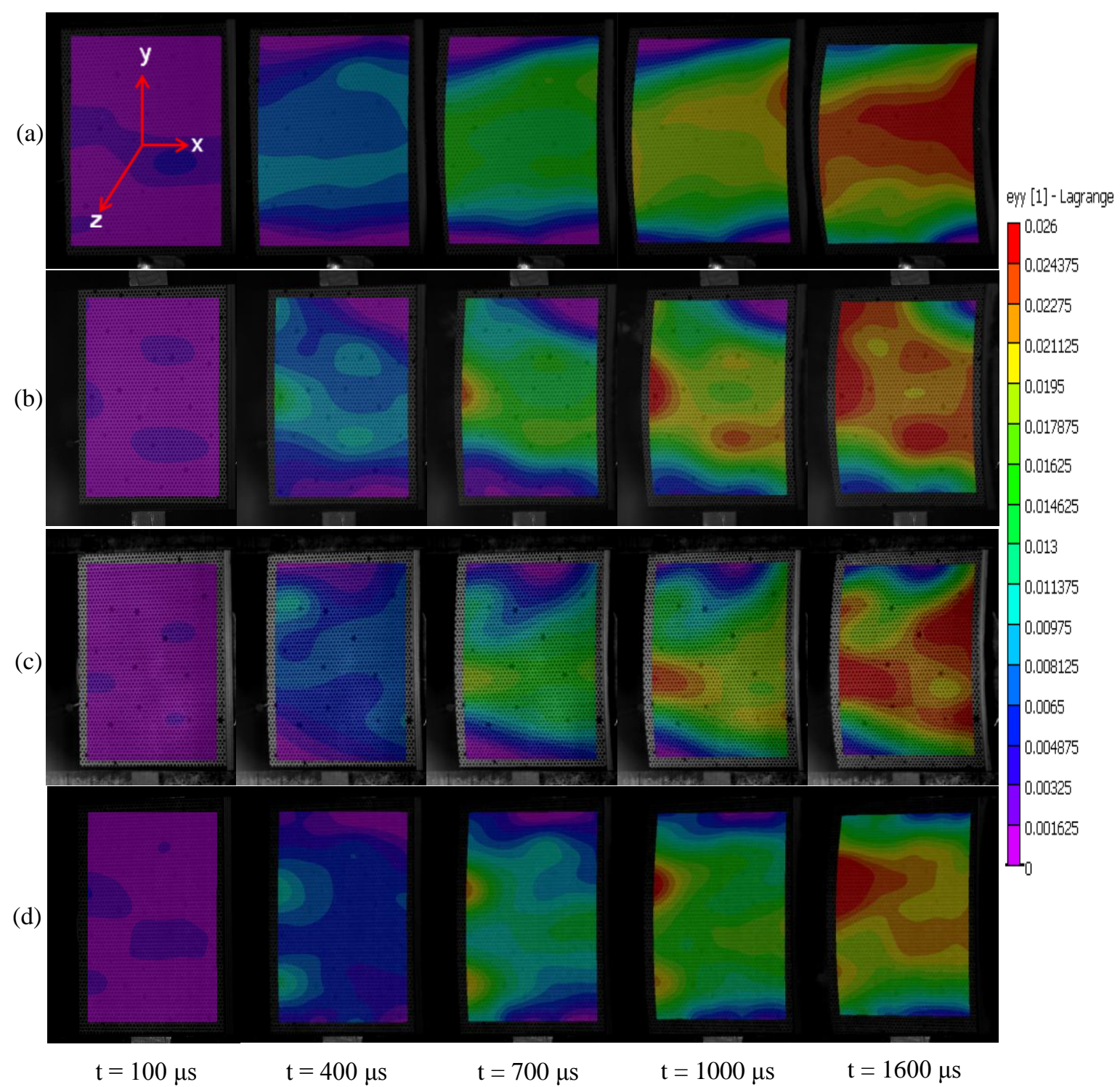

Fig. 15 Full-field in-plane strain ( $\left.\varepsilon_{y y}\right)$ for (a) One layer (b) Two layers

(c) Three layers and (d) Four layers of core gradation

The full-field in-plane strain $\left(\varepsilon_{y y}\right)$ is shown in Fig 15 for all core gradations with a scale of 0 (purple) to .026 (red), or $0 \%$ to $2.6 \%$ respectively. It can be observed in the figure that the back face of all core configurations exhibits very minimal in-plane strain $\left(\varepsilon_{\text {yy }}\right)$ until $\mathrm{t}=100 \mu \mathrm{s}$. Between $\mathrm{t}=100 \mu \mathrm{s}$ and $\mathrm{t}=1600 \mu \mathrm{s}$, all four of these configurations continue bending and the in-plane strain values increase. For the sandwich panel with one layer of core gradation, as shown in Fig. 15a, it can be 
observed that at $\mathrm{t}=1600 \mu \mathrm{s}$, the panel shows a maximum in-plane strain value across the central region of approximately $2.6 \%$. For two, three and four layers of core gradation, as shown in Figures $15 \mathrm{~b}, 15 \mathrm{c}$, and $15 \mathrm{~d}$ respectively, the approximate maximum in-plane strain values across the central region of the panels are not conclusive, but the trend is evident. When using more than one layer of core gradation, it can be seen that the strain distribution is altered, resulting in a reduction of maximum in-plane strain values observed on the back facesheet.

Using the point inspection tool from the Digital Image Correlation (DIC) software, a point directly in the center of the back face of each specimen was chosen. The out-of-plane deflection (W) showed excellent agreement with the results generated utilizing the high-speed images and therefore only the in-plane strain $\left(\varepsilon_{\mathrm{yy}}\right)$ and out-of-plane velocity (dW/dt) results are shown. Fig. 16 and Fig. 17 show the inplane strain and out-of-plane velocity values obtained. Looking at the in-plane strain values (Fig. 16) it can be seen that at $t=1600 \mu$ s, the maximum in-plane strain value at the central point of the back facesheet for one layer core gradation is approximately 2.4\%. When using more layers of core gradation, i.e. two, three and four layers, at $\mathrm{t}=$ $1600 \mu$ s the maximum in-plane strain values are reduced to $2.3 \%, 2.2 \%$ and $2.1 \%$ respectively. Therefore, it can be concluded that using more layers of core gradation, the maximum in-plane strain values are reduced by $4 \%, 8 \%$ and $12.5 \%$ for two, three and four layer gradation in comparison to one layer of core gradation. 


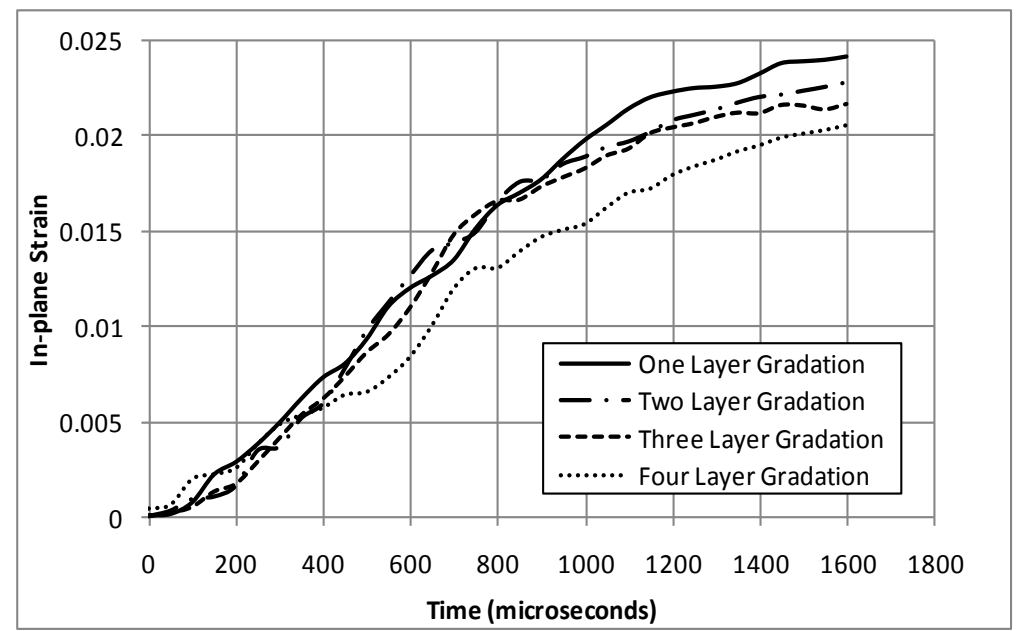

Fig. 16 In-plane strain $\left(\varepsilon_{y y}\right)$ of all core configurations

Fig. 17 shows the out-of-plane velocity (dW/dt) for all core gradations. For one layer of core gradation, the maximum velocity is reached at $\mathrm{t}=500 \mu \mathrm{s}$ and is approximately $32 \mathrm{~m} / \mathrm{s}$. Using two layers of core gradation, the maximum velocity is reached at approximately the same time, $\mathrm{t}=500 \mu \mathrm{s}$, but the velocity is slightly lower $(31 \mathrm{~m} / \mathrm{s})$. For the sandwich composite with three layers of core gradation, the maximum out-of-plane velocity isn't achieved until $\mathrm{t}=600 \mu \mathrm{s}$ and is approximately 29 $\mathrm{m} / \mathrm{s}$. Utilizing four layers of core gradation, the maximum out-of-plane velocity of 27 $\mathrm{m} / \mathrm{s}$ is reached at approximately $\mathrm{t}=700 \mu \mathrm{s}$. Therefore, two things can be concluded when using more layers of core gradation, i.e. two, three and four layers: (1) the maximum value of the out-of-plane velocity is reduced approximately $15 \%$, from 32 $\mathrm{m} / \mathrm{s}$ to $27 \mathrm{~m} / \mathrm{s}$, and (2) the time at which the maximum value of the out-of-plane velocity is achieved is delayed up to $200 \mu \mathrm{s}$. 


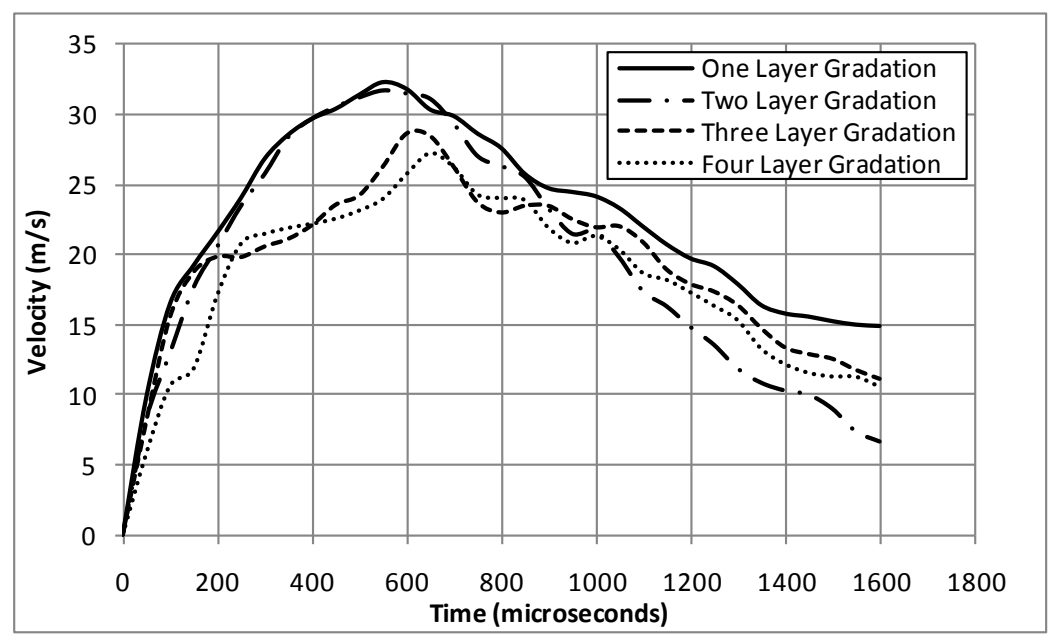

Fig. 17 Out-of-plane velocity (dW/dt) of all core configurations

\subsubsection{Post-mortem Analysis}

After the blast loading event occurred, the damage patterns of the sandwich composites with four different core layer arrangements were visually examined and recorded using a high resolution digital camera and are shown in Fig.18. When the sandwich composite with one layer core gradation was subjected to highly-transient loading, as shown in Fig. 18a, the damage was confined to the areas where the supports were located in the shock tube and core cracking is visible in these two areas. The core cracks propagated completely through the foam core. Core delamination is visible between the two core layers of A500 foam. Also one of the core cracks lead to back skin delamination, where the core separated from the back facesheet. Some core compression is also visible in the first core layer of A500 foam.

For the sandwich composite with two layers of core gradation, the damage patterns after being subjected to the shock loading are shown in Fig. 18b. For this core configuration, the damage was again confined to the areas where the supports were located in the shock tube and core cracking is evident. The core cracks propagated 
completely through the foam core. Skin delamination is obvious between the front facesheet and the foam core, as well as back skin delamination between the back facesheet and the foam core. Core delamination between the first and second core layers of foam, A300 and A800 respectively, is also evident, along with core compression in the first core layer of foam (A300).

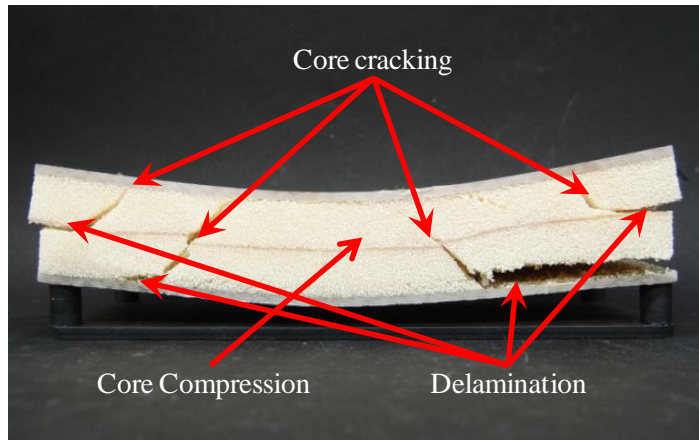

(a) One Layer Gradation

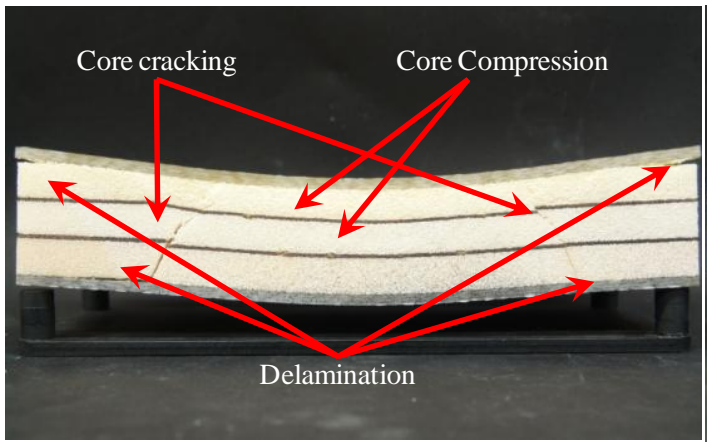

(c) Three Layer Gradation

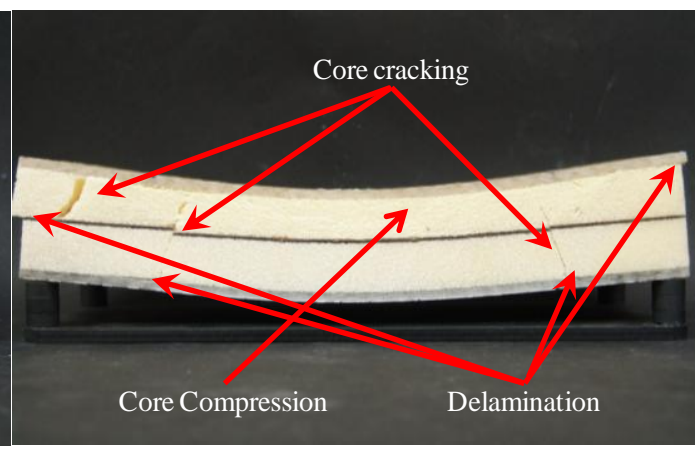

(b) Two Layer Gradation

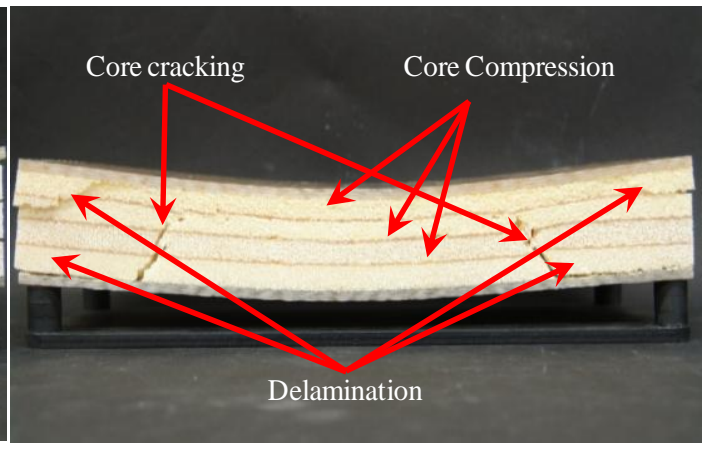

(d) Four Layer Gradation

Fig. 18 Visual examination of all core configurations after being subjected to high-intensity blast loading

Fig. 18c shows the damage patterns of the sandwich composite with three layers of core gradation after the blast loading event occurred. Again, the damage to this core configuration was confined to the areas where the supports were located in the shock tube and core cracking is visible in these two areas. These core cracks propagated completely through the foam core. Also skin delamination is visible between the front facesheet and the foam core, as well as back skin delamination located between the 
back facesheet and the foam core. Core compression is also evident in both the first and second core layers of foam, A300 and A500 respectively.

When the sandwich composite with four layers of core gradation was subjected to the shock loading, as shown in Fig. 18d, the damage was again confined to the areas where the supports from the shock tube were located and core cracking is evident in these two areas. Unlike the previous three configurations, the core cracks did not propagate completely through the foam core. Core delamination is obvious between the first and second core layers of foam, A300 and A400, as well as back skin delamination between the back facesheet and the foam core. Core compression is very obvious in this configuration. The first, second and third layer of foam, A300, A400 and A500 respectively, all exhibit various amounts of core compression.

\subsubsection{Permanent Deformation}

The permanent deflection (deformation) for each graded core configuration was measured after the shock loading experiment. A schematic of the specimen and how the measurements were taken can be seen in Fig. 19. The distance between the top dotted line (red) and the front surface of the front facesheet is defined as the permanent deflection of the front face. Similarly, the distance between the bottom dotted line and the top surface of the back facesheet is defined as the permanent deflection of the back face. Subtracting the total permanent deflection of the back face from the front face, the final core thickness and thus total core compression (permanent) can be obtained. These values are shown in Table 5 . 


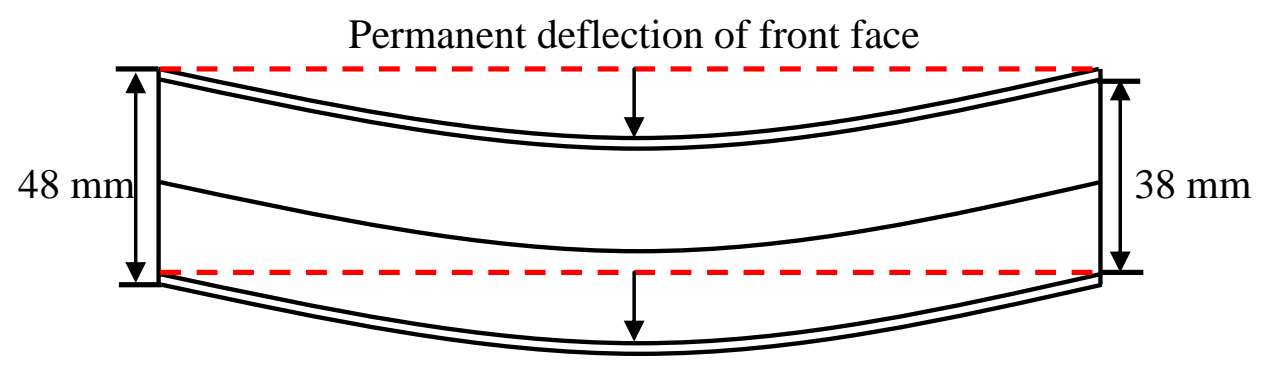

Permanent deflection of back face

Fig. 19 Specimen schematic for permanent deflection

For one layer core gradation, the front face exhibited a permanent deflection of $\sim 11.1 \mathrm{~mm}$, while the back face exhibited $\sim 8.5 \mathrm{~mm}$. By subtracting the back face deflection from the font face deflection, the total amount of permanent core compression can be calculated. For the sandwich panel with one layer of core gradation, the core showed approximately $2.6 \mathrm{~mm}(7 \%)$ of permanent core compression. When using two layers of core gradation, the front face showed $\sim 14.1$ $\mathrm{mm}$ of permanent deflection, while the back face showed approximately $\sim 9.2 \mathrm{~mm}$. Therefore, it can be concluded that the foam core itself exhibited approximately 4.9 $\mathrm{mm}(13 \%)$ of permanent compression. For three layers of core gradation, the front face exhibited $\sim 12.9 \mathrm{~mm}$ of permanent deflection, while the back face exhibited $~ 7.3$. This results in a total core compression of approximately $5.6 \mathrm{~mm}(15 \%)$. When using four layers of core gradation, the front face shows approximately $12.4 \mathrm{~mm}$ of permanent deflection, while the back face shows $6.1 \mathrm{~mm}$. Consequently, the total permanent compression of the core is $6.3 \mathrm{~mm}(17 \%)$. Therefore, increasing the number of monotonically graded core layers results in higher levels of permanent core compression, but lower levels of permanent back face deflections. 
The total core compression observed during the experiment, as measured from the high-speed images and shown in Fig. 10 is listed in Table 5 (Real-time core compression). Subtracting the total permanent core compression from the real-time core compression, the total amount of core compression recovered can be measured. When using one and two layers of core gradation, the amount of core compression recovered is almost the same, approximately $8.4 \mathrm{~mm}$ and $8.1 \mathrm{~mm}$ respectively. When using three and four layers of core gradation, the total amount of core compression recovered is $9.4 \mathrm{~mm}$ and $10.7 \mathrm{~mm}$. Thus, higher levels of core gradation allow for larger amounts of real-time compression to be recovered. This can be directly related to the strain-time history data in Fig. 11 and discussed in Section 4.2.2. When using higher levels of core gradation, the maximum strain values achieved in the same individual foam core layers was reduced. For the A300 foam core layer, the maximum amount of strain was reduced from approximately $80 \%$ for two layer gradation, to $70 \%$ and $65 \%$ for three and four layers of core gradation respectively. For the A500 foam core layer, the maximum amount of strain was reduced from $50 \%$ for three layers gradation to $30 \%$ for four layer of core gradation. Therefore, with higher levels of core gradation, the same individual foam core layers exhibit less strain, thus allowing for more real-time core compression to be restored post-blast.

The post-mortem images in Fig. 18, along with the results generated in Table 5, indicate the ability of functionally graded sandwich composites with higher levels of core gradation (three and four) to better mitigate blast energy. The sandwich panels with three and four layers of core gradation exhibited the largest amount of total core compression, $5.6 \mathrm{~mm}(15 \%)$ and $6.3 \mathrm{~mm}(17 \%)$, but the least amount of permanent 
back face deflection, $7.3 \mathrm{~mm}$ and $6.1 \mathrm{~mm}$. Also, these sandwich panels showed the largest amount of real-time core compression recovered, $9.4 \mathrm{~mm} \mathrm{(25 \% )} \mathrm{and} 10.7 \mathrm{~mm}$ $(28 \%)$.

Table 5. Permanent deflection and core compression

\begin{tabular}{ccccccc}
\hline $\begin{array}{c}\text { Core } \\
\text { Gradation }\end{array}$ & $\begin{array}{c}\text { Permanent } \\
\text { Front Face } \\
\text { Deflection } \\
(\mathrm{mm})\end{array}$ & $\begin{array}{c}\text { Permanent } \\
\text { Back Face } \\
\text { Deflection } \\
(\mathrm{mm})\end{array}$ & $\begin{array}{c}\text { Final } \\
\text { Core } \\
\text { Thickness } \\
(\mathrm{mm})\end{array}$ & $\begin{array}{c}\text { Permanent } \\
\text { Core } \\
\text { Compression } \\
(\mathrm{mm} / \%)\end{array}$ & $\begin{array}{c}\text { Real-Time } \\
\text { Core } \\
\text { Compression } \\
(\mathrm{mm} / \%)\end{array}$ & $\begin{array}{c}\text { Recovered } \\
(\mathrm{mm} / \%)\end{array}$ \\
\hline 1 Layer & 11.1 & 8.5 & 35.4 & $2.6(7 \%)$ & $11(30 \%)$ & $8.4(23 \%)$ \\
2 Layer & 14.1 & 9.2 & 33.1 & $4.9(13 \%)$ & $13(35 \%)$ & $8.1(22 \%)$ \\
3 Layer & 12.9 & 7.3 & 32.4 & $5.6(15 \%)$ & $15(40 \%)$ & $9.4(25 \%)$ \\
4 Layer & 12.4 & 6.1 & 31.7 & $6.3(17 \%)$ & $17(45 \%)$ & $10.7(28 \%)$ \\
\hline
\end{tabular}

\section{Discussion on Stress Wave Propagation in Functionally Graded/Layered}

\section{Materials}

The stress wave propagation in functionally graded/layered materials has been extensively investigated by [23 - 29]. Note for these investigations the wave interactions within the specimens were treated as one-dimensional, and without dispersion and compression waves. Makris et al. and Nerenberg et al. [23, 24] studied the attenuation of a blast wave with a cellular material (polymeric foam) and found that if a foam layer is loaded by a decaying blast wave, as opposed to a shock wave with a constant pressure profile, the shock wave transmitted through the foam may either be amplified or attenuated, depending on the blast wave strength and duration as well as the foam stiffness and thickness. Attention must be paid to the sequence and ordering of the layers, which introduces interfaces and affects the stress transmission greatly [25]. The stress at an interface with a large variation in impedances will mainly 
be governed by the properties of the material with lower impedance. Similarly, the equilibrium motion of the interface is governed by the material with high impedance [26]. Changjing et al. [27] found that during dynamic compression, the increase in the acoustic impedance should be small, and a better matching of wave impedance for layered media can change the stress peak value, duration, and energy distribution based on the consideration of wave attenuation and energy dissipation.

These results demonstrated that combining materials with pronounced differences in material properties, in an optimal configuration and arrangement, can lead to efficient wave attenuation. There are two ways in which the peak pressure of a compression or shock wave can be attenuated: through expansion of the high pressure shock wave (energy dissipation mechanism) or through scattering/dispersion of the wave through interface variations. During blast wave loading, the expansion wave following the shock wave is the main cause of the attenuation. As the rarefraction wavelets propagate faster in the compacted foam than the shock wave in the porous foam, the expansion fan is able to catch up to the shock wave and attenuate it gradually in a sufficiently thick foam layer $[23,24]$. By altering the number of interfaces and distribution of materials within a composite structure, the wave decoupling (scattering/dispersion) effect could be optimized. In other words, as the number of interfaces is increased, the number of wave reflections within the material system is greatly increased as well. These reflections lengthen the timescales for pressure rise across the sample $[26,28]$ allowing for a time-delay of the peak stress arrival, which in turn delays the time of damage initiation [29]. 
Even though it is nearly impossible to conclude which mechanism had a more important role in attenuating the stress wave, thus improving the dynamic performance of the structure when subjected to a blast loading, we do know that both mechanisms played a vital role. By inspecting the high-speed images in Fig. 9 and deflection plots in Fig 10, it can be observed that increasing the number of core layers allows for a stepwise compression of the core (energy dissipation mechanism). The scattering method of wave attenuation (time-delay phenomena) can be observed in the out-ofplane velocity (dW/dt), Fig. 17, as well as the high-speed images, Fig. 9. When using three and four layers of core gradation, the maximum velocities are reached $100 \mu \mathrm{s}$ and $200 \mu$ s respectively after the sandwich composites with one and two layers of core gradation (Fig. 17). This time-delay in the maximum velocity in turn delays the time of damage initiation, as seen in Fig. 9 and discussed in Section 4.2.1. When using more layers of core gradation (three and four), and thus introducing more material interfaces, the onset of the fourth damage zone was delayed $(\sim 200 \mu \mathrm{s})$.

\section{Conclusions}

The following is the summary of the investigation:

(1) The dynamic stress-strain response is significantly higher than the quasi-static response for every type of Corecell ${ }^{\mathrm{TM}} \mathrm{A}$ foam studied. Both quasi-static and dynamic constitutive behaviors of Corecell ${ }^{\mathrm{TM}}$ A series foams (A300, A400, A500, and A800) show an increasing trend. The increase in the yield strength from quasi-static response to dynamic response, along with the longer stress plateau, indicates that these foam materials show great potential in absorbing large amounts of energy. 
(2) Sandwich composites with four different core layer arrangements, one, two, three and four layers respectively, were subjected to shock wave loading. The overall performance of the sandwich composite with four layers of core gradation is the best, followed by the sandwich composites with three, two and one layer gradation respectively. Even though each configuration allowed for a stepwise compression of the core, it was shown that the number of core layers has an influence on the dynamic response of the structure under blast loading. More specifically, by increasing the number of monotonically graded layers, the acoustic wave impedance mismatch between successive layers is reduced. Therefore, the strength of the initial shock wave (stress wave) can be weakened by the time it reaches the back facesheet, resulting in lower back face deflection, in-plane strain, and velocity. More importantly, the overall damage imparted on the structure can be reduced and structural integrity can be maintained.

(3) Increasing the number of monotonically graded foam core layers, thus introducing more material interfaces, allows for blast wave (stress wave) attenuation through the following mechanisms: (1) stepwise compression of the core (energy dissipation mechanism) and (2) scattering/dispersion of the wave through interface variations. Combining these mechanisms results in lengthened timescales for pressure rises across the samples, allowing for a time-delay of the peak stress arrival, and thus delaying the time of damage initiation. 
(4) When using higher levels of core gradation, i.e. two, three and four layers respectively, the amount of stress transferred to subsequent layers is diminished, thereby subjecting the back face to reduced loadings and blast pressures.

\section{Acknowledgements}

The authors kindly acknowledge the financial support provided by Dr. Yapa D. S. Rajapakse, under Office of Naval Research (ONR) Grant No. N00014-10-1-0662. The authors acknowledge the support provided by the Department of Homeland Security (DHS) under Cooperative Agreement No. 2008-ST-061-ED0002. Authors thank Gurit SP Technology for providing the material, as well as Dr. Stephen Nolet and TPI Composites for providing the facility for creating the composites used in this study. A special thanks to Dr. Richard Brown for providing the Scanning Electron Microscopy (SEM) facility used in obtaining the images of the cell microstructures.

\section{References}

[1] Xue Z, Hutchinson JW. Preliminary assessment of sandwich plates subject to blast loads. International Journal of Mechanical Sciences 2003; 45: 687-705.

[2] Fleck NA, Deshpande VS. The resistance of clamped sandwich beams to shock loading. Journal of Applied Mechanics 2004; 71: 386-401.

[3] Dharmasena KP, Wadley HNG, Xue Z, Hutchinson JW. Mechanical response of metallic honeycomb sandwich panel structures to high-intensity dynamic loading. International Journal of Impact Engineering 2008; 35(9): 1063-1074.

[4] Zhu F, Zhao L, Lu G, Wang Z. Deformation and failure of blast loaded metallic sandwich panels - Experimental investigations. International Journal of Impact Engineering 2008; 35(8): 937-951. 
[5] Nurick GN, Langdon GS, Chi Y, Jacob N. Behavior of sandwich panels subjected to intense air blast: part 1- Experiments. Composite Structures 2009; 91 (4): 433-441.

[6] Radford DD, Fleck NA, Deshpande VS. The response of clamped sandwich beams subjected to shock loading. International Journal of Impact Engineering 2004; 32(6): 968-987.

[7] Wang Z, Jing L, Ning J, Zhao L. The structural response of clamped sandwich beams subjected to impact loading. Composite Structures 2011; 93: 1300-1308.

[8] Tagarielli VL, Deshpande VS, Fleck NA. The high strain rate response of PVC foams and end-grain balsa wood. Composites: Part B 2008; 39: 83-91.

[9] Atas C, Sevim C. On the impact response of sandwich composites with cores of balsa wood and PVC foam. Composite Structures 2010; 93: 40-48.

[10] McShane GJ, Deshpande VS, Fleck NA. The underwater blast resistance of metallic sandwich beams with prismatic lattice cores. Journal of Applied Mechanics 2007; 74: 352-364.

[11] McShane GJ, Radford DD, Deshpande VS, Fleck NA. The response of clamped sandwich plates with lattice cores subjected to shock loading. European Journal of Mechanics - A: Solids 2006; 25: 215-229.

[12] Hossain MK, Liu QL, O’Toole BJ. Functionally graded foam material system for energy absorption. SAMPE 39 ${ }^{\text {th }}$ ISTC- Cincinnati OH, Oct. 29 - Nov. 1, 2007.

[13] Kiernan S, Cui L, Gilchrist MD. Propagation of a stress wave through a virtual functionally graded foam. International Journal of Non-Linear Mechanics 2009; 44: 456-468.

[14] Cui L, Kiernan S, Gilchrist MD. Designing the energy absorption capacity of functionally graded foam materials. Materials Science and Engineering: A 2009; 507: 215-225.

[15] Li Y, Ramesh, KT, Chin ESC. Dynamic characterization of layered and graded structures under impulsive loading. International Journal of Solids and Structures 2001; 38(34-35): 6045-6061. 
[16] Apetre NA, Sankar BV, Ambur DR. Low-velocity impact response of sandwich beams with functionally graded core. International Journal of Solids and Structures 2006; 43(9): 2479-2496.

[17] Wang E, Gardner N, Shukla A. The blast resistance of sandwich composites with stepwise graded cores. International Journal of Solids and Structures 2009; 46: 34923502 .

[18] http://www.gurit.com/corecell-afoam.aspx. Accessed 20 Sept 2011.

[19] Gardner N, Wang E, Kumar P, Shukla A. Blast mitigation in a sandwich composite using graded core with polyurea interlayer. Experimental Mechanics 2011. DOI 10.1007/s11340-011-9517-9.

[20] Chen W, Zhang B, Forrestal MJ. A split Hopkinson bar technique for low impedance materials. Experimental Mechanics 1998; 39 (2): 81-85.

[21] Gibson LJ, Ashby MF. Cellular Solids: Structures and Properties. Elmsford, NY: Pergamon Press, Inc., 1988.

[22] Wang, E. Blast resistance and energy mitigation behavior of sandwich composite materials. Ph.D Dissertation, University of Rhode Island, 2010.

[23] Makris A, Frost DL, Nerenberg J, Lee JHS. Attenuation of a blast wave with a cellular material. In: Sturtevant B, Shepard JE, Hornung HG, editors. Proceedings of the $20^{\text {th }}$ International Symposium on Shock Waves (ISSW/20), Pasadena, CA, 1996. World Scientific. Vol. II, p.1387-1392.

[24] Nerenberg J, Nemes JA, Frost DL, Makris A. Blast wave loading of polymeric foam, In: Houwing AFP et al., editors. Proceedings of the $21^{\text {st }}$ International Symposium on Shock Waves (ISSW/21), Australia, 20-25 July 1997. Panther Publishing and Printing, Vol. I, p. 91-96.

[25] Wang LL. Foundation of stress waves, Elsevier, 2007.

[26] Petel OE, Jette FX, Goroshin S, Frost DL, Ouellet, S. Blast wave attenuation through a composite of varying layer distribution. Shock Waves 2011. DOI 10.1007/s00193-010-0295-6. 
[27] Changjing X, Yongxiang D, Liping C, Dongjie X, and Shunhong W. Propagation characteristic of explosive waves in layered media: Numerical analysis. Defence Science Journal 2009; 59(5): 499-504.

[28] Bruck H. A one-dimensional model for designing functionally graded materials to manage stress waves. International Journal of Solids and Structures 2000; 37: 63836395 .

[29] Samadhiya R, Mukherjee A, and Schmauder S. Characterization of discretely graded materials using acoustic wave propagation. Computational Materials Science 2006; 37: 20-28. 


\title{
CHAPTER 4
}

\section{BLAST MITIGATION IN A SANDWICH COMPOSITE USING A GRADED CORE AND POLYUREA INTERLAYER}

\author{
by \\ Nate Gardner, Erheng Wang, Puneet Kumar and Arun Shukla \\ has been published in Experimental Mechanics (2012, Vol. 52, 119-133) \\ Corresponding Author: Arun Shukla \\ Dynamic Photo Mechanics Laboratory \\ Department of Mechanical, Industrial and Systems \\ Engineering \\ University of Rhode Island \\ 206 Wales Hall, 92 Upper College Rd \\ Kingston, RI, 02881, USA \\ Phone: +1-401-874-2283 \\ Email Address: shuklaa@egr.uri.edu
}




\begin{abstract}
The dynamic behavior of two types of sandwich composites made of E-glass Vinyl-

Ester (EVE) facesheets and Corecell ${ }^{\mathrm{TM}} \mathrm{A}$-series foam with a polyurea interlayer was studied using a shock tube apparatus. The materials, as well as the core layer arrangements, were identical, with the only difference arising in the location of the polyurea interlayer. The foam core itself was layered with monotonically increasing wave impedance of the core layers, with the lowest wave impedance facing the shock loading. For configuration 1, the polyurea interlayer was placed behind the front facesheet, in front of the foam core, while in configuration 2 it was placed behind the foam core, in front of the back facesheet. A high-speed side-view camera, along with a high-speed back-view 3-D Digital Image Correlation (DIC) system, was utilized to capture the real-time deformation process as well as mechanisms of failure. Postmortem analysis was also carried out to evaluate the overall blast performance of these two configurations. The results indicated that applying polyurea behind the foam core and in front of the back facesheet will reduce the back face deflection, particle velocity, and in-plane strain, thus improving the overall blast performance and maintaining structural integrity.
\end{abstract}

Keywords: Sandwich Structures, Functionally Graded Material, Polyurea Interlayer, Blast Loading, High-speed Photography

\title{
1. Introduction
}

Core materials play a crucial role in the dynamic behavior of sandwich structures when they are subjected to high-intensity impulse loadings such as air blasts. Their 
properties assist in dispersing the mechanical impulse that is transmitted into the structure and thus protect anything located behind it [1-3]. Stepwise graded materials, where the material properties vary gradually or layer by layer within the material itself, were utilized as a core material in sandwich composites due to the fact that their properties can be designed and controlled. Typical core materials utilized in blast loading applications are generally foam, due to their ability to compress and withstand highly transient loadings. In recent years, with its ability to improve structural performance and damage resistance of structures, as well as effectively dissipate blast energy, the application of polyurea to sandwich structures has become a new area of interest

The numerical investigation by Apetre et al. [4] on the impact damage of sandwich structures with a graded core (density) has shown that a reasonable core design can effectively reduce the shear forces and strains within the structures. Consequently, they can mitigate or completely prevent impact damage on sandwich composites. Li et al. [5] examined the impact response of layered and graded metalceramic structures numerically. He found that the choice of gradation has a great significance on the impact applications and the particular design can exhibit better energy dissipation properties. In their previous work, the authors experimentally investigated the blast resistance of sandwich composites with stepwise graded foam cores [6]. Two types of core configurations were studied and the sandwich composites were layered / graded based on the densities of the given foams, i.e. monotonically and non-monotonically. The results indicated that monotonically increasing the wave impedance of the foam core, thus reducing the wave impedance mismatch between 
successive foam layers, will introduce a stepwise core compression, greatly enhancing the overall blast resistance of sandwich composites.

Although the behavior of polyurea has been investigated [7-10], there have been no studies regarding the dynamic behavior of functionally graded core with a polyurea interlayer. Tekalur et al. [11] experimentally studied the blast resistance and response of polyurea based layered composite materials subjected to blast loading. Results indicated that sandwich materials prepared by sandwiching the polyurea between two composite skins had the best blast resistance compared to the EVE composite and polyurea layered plates. Bahei-El-Din et al. [12] numerically investigated the blast resistance of sandwich plates with a polyurea interlayer under blast loading. Their results suggest that separating the composite facesheet from the foam core by a thin interlayer of polyurea can be very beneficial in comparison to the conventional sandwich plate design. Amini et al. [13 - 16] experimentally and numerically studied the dynamic response of circular monolithic steel and steel-polyurea bilayer plates to impulsive loads. More importantly they focused on the significance of the relative position of the polyurea layer with respect to the loading direction. Results indicated that the polyurea layer can have a significant effect on the response of the steel plate to dynamic impulsive loads, both in terms of failure mitigation and energy absorption, if it is placed on the back face of the plate. On the contrary, they also found that polyurea can enhance the destructive effect of the blast, promoting (rather than mitigating) the failure of the steel plate if applied on the impact side of the plate.

The present study focuses on the blast response of sandwich composites with a functionally graded core and a polyurea (PU) interlayer. Two different core layer 
configurations were investigated, with the only difference arising in the location of the polyurea (PU) interlayer. The quasi-static and dynamic constitutive behaviors of the foam core materials, as well as the polyurea, were first experimentally studied using a modified SHPB device with a hollow transmitted bar. The sandwich composites were then subjected to shock wave loading generated by a shock tube. The shock pressure profiles, real-time deflection images, and post-mortem images were carefully analyzed to reveal the mechanisms of dynamic failure of these sandwich composites. Digital Image Correlation (DIC) analysis was implemented to investigate the real-time deflection, strain, and particle velocity.

\section{Material and Specimen}

\subsection{Skin and Core Materials}

The skin materials that were utilized in this study are E-glass Vinyl-Ester (EVE) composites. The woven roving E-glass fibers of the skin material were placed in a quasi-isotropic layout $[0 / 45 / 90 /-45]_{\mathrm{s}}$. The fibers were made of the $0.61 \mathrm{~kg} / \mathrm{m}^{2}$ areal density plain weave. The resin system used was Ashland Derakane Momentum 8084, with the front and back skins (facesheets) consisting of identical layups and materials. Fig. 1 shows a schematic of the sandwich composite with skin and core materials.

The foam core materials used in the present study are Corecell ${ }^{\mathrm{TM}} \mathrm{A}$-series styrene acrylonitrile (SAN) foams, which are manufactured by Gurit SP Technologies specifically for marine sandwich composite applications. The three types of Corecell $^{\mathrm{TM}}$ A-series foam that were used in present study were A300, A500, and A800. The polyurea used in the present study is Dragonshield-HT, which is manufactured by Specialty Products Incorporated (SPI) specifically for blast resistance 
and mitigation. It is a state-of-the-art high performance, sprayed, plural component pure elastomer, based on amine-terminated polyether resins, amine chain extenders and MDI prepolymers. Table 1 lists important material properties from the manufacturer's data of the three foams [17], as well as the Dragonshield-HT polyurea [18] and the material properties of the facesheet as determined using ASTM standards. In Table 1, the A300 foam has the lowest nominal density $(\rho)$, as well as compressive modulus (E) of the three foams, followed by the A500 and A800 foams respectively. Since both the nominal density and the compressive modulus are increasing from A300 to A800 foam, the wave impedance also increases.

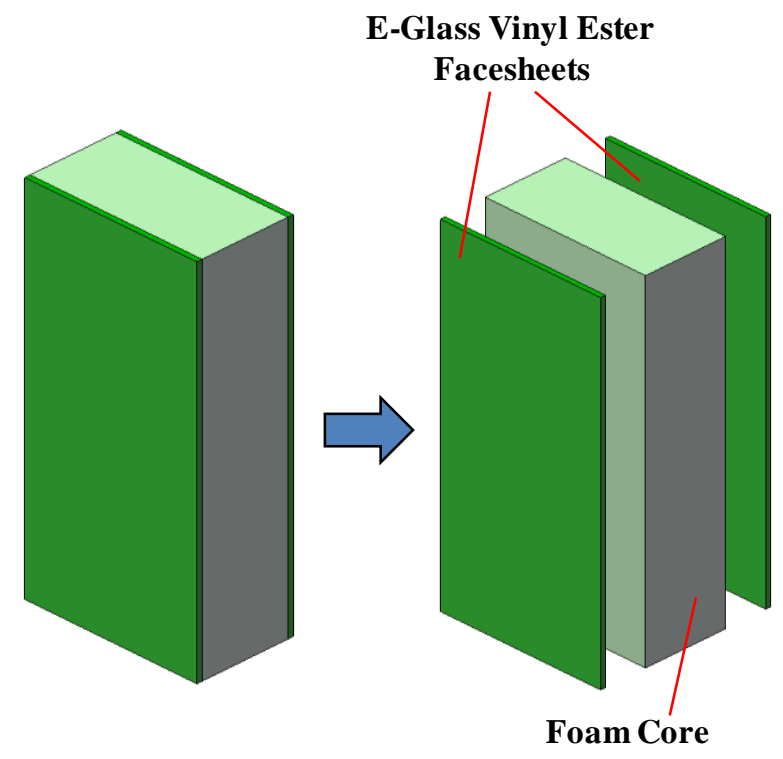

Fig. 1 Schematic of sandwich composite with skin and core

The cell structures for the three foams are very similar and the only difference appears in the cell wall thickness and node sizes, which accounts for the different densities of the foams. The SEM images of the cell microstructures can be seen in Fig. 2. 
Table 1 Material properties for foam core [17] and polyurea [18]

\begin{tabular}{ccccc}
\hline & $\begin{array}{c}\text { Nominal } \\
\text { Density, } \boldsymbol{\rho} \\
\left(\mathrm{kg} / \mathrm{m}^{3}\right)\end{array}$ & $\begin{array}{c}\text { Compressive } \\
\text { Modulus, E } \\
(\mathrm{MPa})\end{array}$ & $\begin{array}{c}\text { Compressive } \\
\text { Strength, } \boldsymbol{\sigma}_{\mathbf{y}} \\
(\mathrm{MPa})\end{array}$ & $\begin{array}{c}\text { Elongation } \\
(\%)\end{array}$ \\
\hline $\mathbf{A 3 0 0}$ & 58.5 & 32 & 0.5 & - \\
$\mathbf{A 5 0 0}$ & 92 & 64 & 0.9 & - \\
$\mathbf{A 8 0 0}$ & 150 & 117 & 2.1 & - \\
Dragonshield-HT & 1000 & - & - & - \\
$\begin{array}{c}\text { E-glass Vinyl- } \\
\text { Ester Composite }\end{array}$ & 1800 & 13600 & 220 & \\
\hline
\end{tabular}

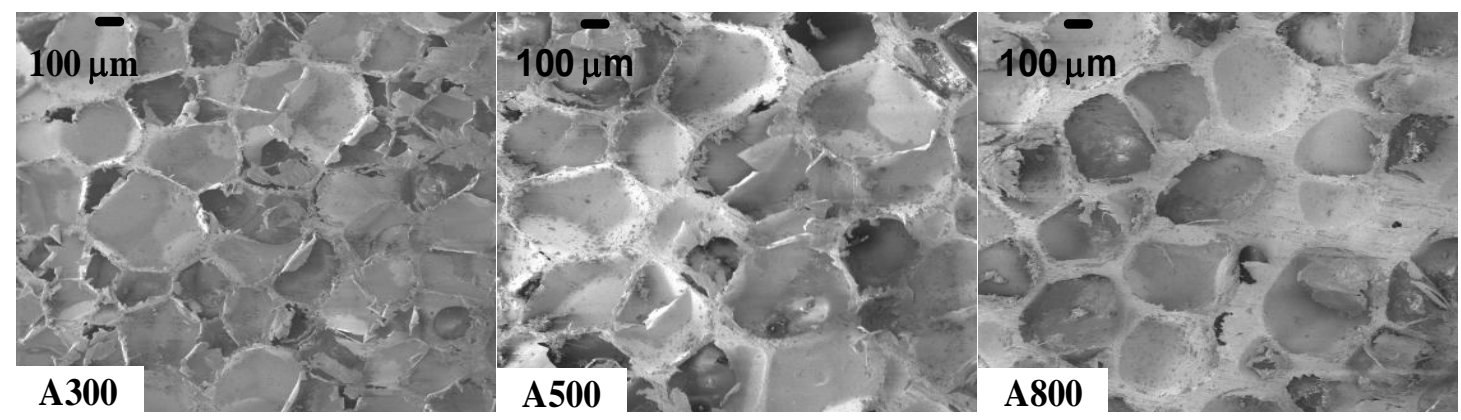

Fig. 2 Cell microstructure of foam core layers

\subsection{Sandwich Panels with Stepwise Graded Core Layer Arrangement and}

\section{Polyurea (PU) Interlayer}

The Vacuum Assisted Resin Transfer Molding (VARTM) process was utilized to fabricate the sandwich specimens. The overall dimensions for the samples were 102 $\mathrm{mm}$ wide, $254 \mathrm{~mm}$ long and $48 \mathrm{~mm}$ thick. The total thickness of the core was $38 \mathrm{~mm}$, with a skin thickness of $5 \mathrm{~mm}$. The core consisted of three layers of foam (A300/A500/A800 - low/middle/high density) and a polyurea (PU) interlayer. The first two layers of the foam core (A300 / A500) were $12.7 \mathrm{~mm}$ thick, while the third 
foam layer (A800) was $6.35 \mathrm{~mm}$. The polyurea interlayer was $6.35 \mathrm{~mm}$. The average areal density of the samples was $26 \mathrm{~kg} / \mathrm{m}^{2}$.

Two core configurations, which consisted of identical core materials, were studied (as shown in Fig. 3a). For configuration 1, the polyurea interlayer was placed behind the front facesheet and in front of the foam core (PU/A300/A500/A800). For configuration 2, the polyurea interlayer was placed behind the foam core, and in front of the back facesheet (A300/A500/A800/PU). With these configurations it should be noted that the first core layer is the one first subjected to the shock wave loading. Actual samples can be seen in Fig.3b.

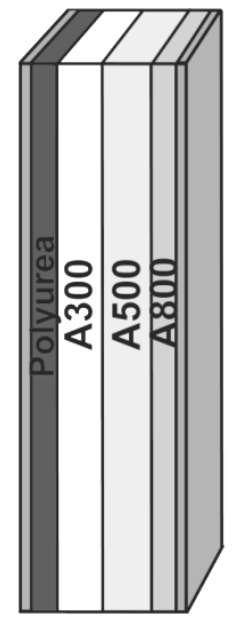

Configuration 1 Configuration 2

(a) Specimen schematics

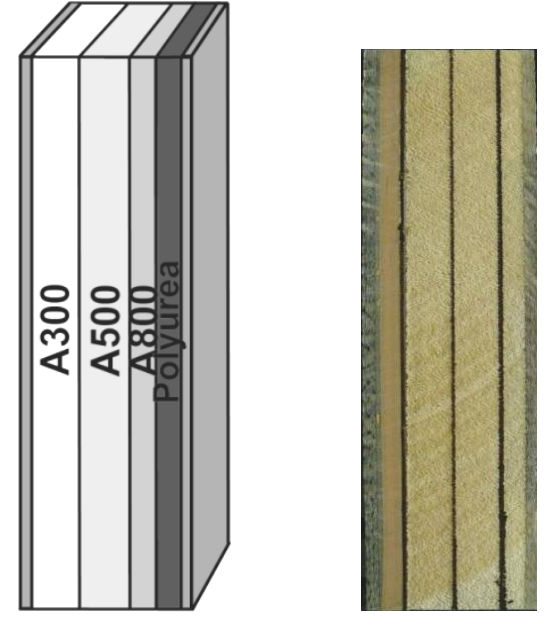

Configuration 1 Configuration 2

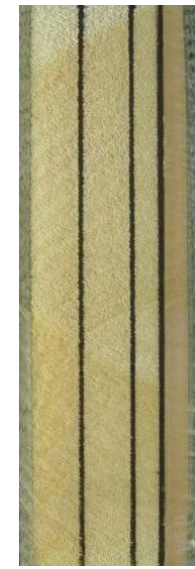

(b) Real specimens

Fig. 3 Specimen configuration and core gradation

\section{Experimental Set-up and Procedure}

\subsection{Modified Split Hopkinson Pressure Bar with Hollow Transmission Bar}

A Split Hopkinson Pressure Bar (SHPB) is the most common device for measuring dynamic constitutive properties of materials. Due to the low-impedance of Corecell $^{\mathrm{TM}}$ foam materials, dynamic experiments for the core materials were performed with a modified SHPB device with a hollow transmission bar to increase 
the transmitted signal intensity. A sketch of the modified SHPB device and typical pulse profiles are given in Fig. 4. It has a $304.8 \mathrm{~mm}$-long striker, $1600 \mathrm{~mm}$-long incident bar and $1447 \mathrm{~mm}$-long transmission bar. All of the bars are made of a 6061 aluminum alloy. The nominal outer diameters of the solid incident bar and hollow transmission bar are $19.05 \mathrm{~mm}$. The hollow transmission bar has a $16.51 \mathrm{~mm}$ inner diameter. At the head and at the end of the hollow transmission bar, end caps made of the same material as the bar were press fitted into the hollow tube. By applying pulse shapers, the effect of the end caps on the stress waves can be minimized. The details of the analysis and derivation of equations for analysis of experimental data can be found in ref [19].

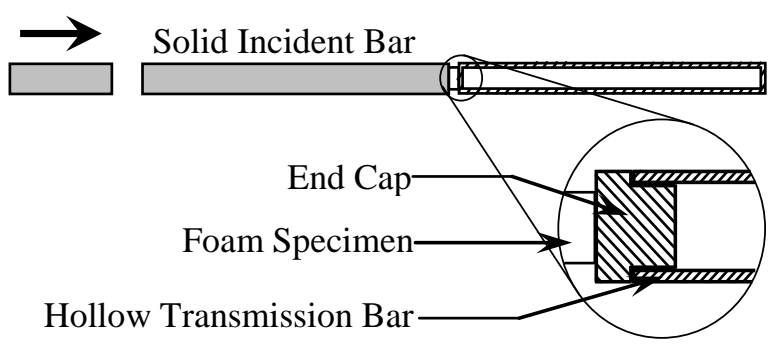

(a) Modified SHPB device

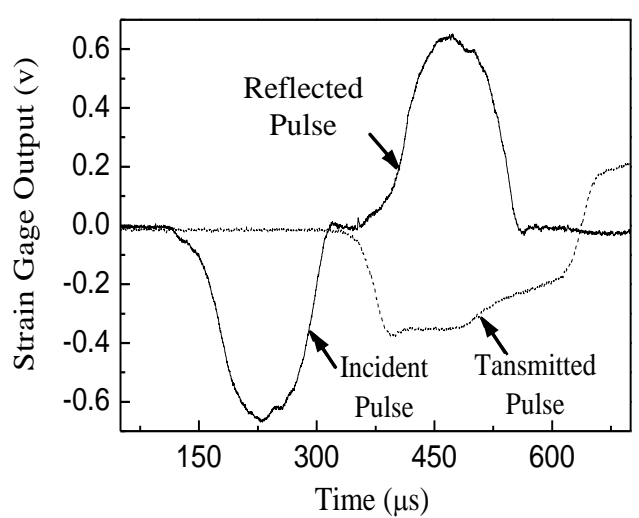

(b) Typical pulse profiles

Fig. 4 Sketch of modified SHPB device with hollow transmission bar and typical pulse profiles

\subsection{Shock Tube}

The shock tube apparatus was utilized to obtain the controlled blast loading (Fig. 5a). It has an overall length of $8 \mathrm{~m}$, consisting of a driver, driven and muzzle section. The high-pressure driver section and the low pressure driven section are separated by a diaphragm. By pressurizing the high-pressure section, a pressure difference across the diaphragm is created. When this difference reaches a critical value, the diaphragms 
rupture. This rapid release of gas creates a pressure wave that develops into a shock wave as it travels down the tube to impart dynamic loading on the specimen.

Fig. 5b shows detailed dimensions and locations of the muzzle, specimen, supports and the pressure sensors (PCB102A). The sensors are mounted at the end of the muzzle section to measure the incident pressure and the reflected pressure during the experiment. The final muzzle diameter is $0.0762 \mathrm{~m}$. The distance between the two sensors is $0.16 \mathrm{~m}$ and the distance between the second sensor and the end of the muzzle is $\sim 0.02 \mathrm{~m}$. The specimen was placed in the supports and positioned close to the end of the muzzle. These support fixtures ensure simply supported boundary conditions with a $0.1524 \mathrm{~m}$ span.

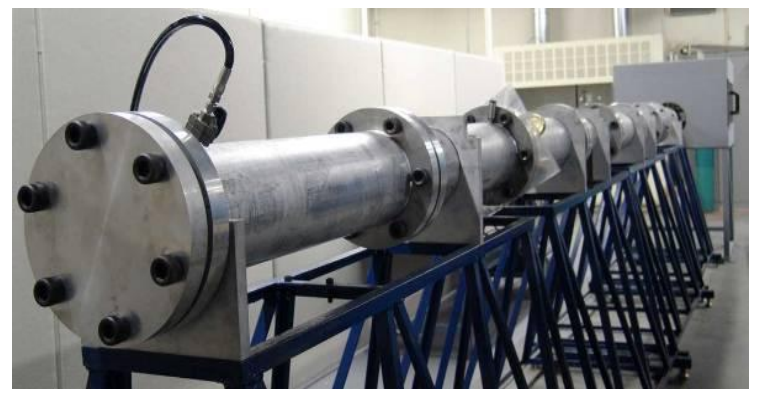

(a) Shock tube

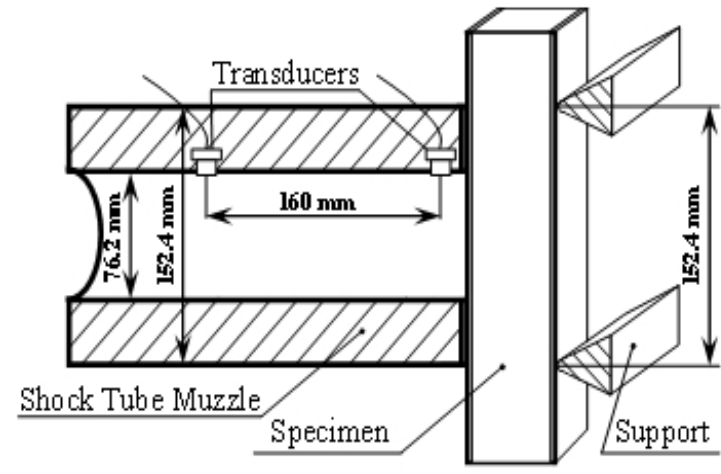

(b) Detailed dimensions of the muzzle

Fig. 5 Shock tube apparatus

\subsection{High-speed Photography Systems}

Two high-speed photography systems were used in the present study, as shown in Fig. 6. A high-speed 3-D Digital Image Correlation (DIC) system, consisting of two high-speed digital cameras [Photron SA1], was placed on the back side of the specimen to obtain the- real-time full-field in-plane and out-of-plane strain, along with deflection and velocity of the back facesheet. A speckle pattern was placed directly on 
the back facesheet of the sandwich composite to ensure good contrast of the images. Another high-speed digital camera, [Photron SA1], was placed perpendicular to the side surface of the specimen to capture the side-view deformation images. A framing rate of 20,000 fps was utilized which gives an interframe time of approximately $50 \mu$ s.

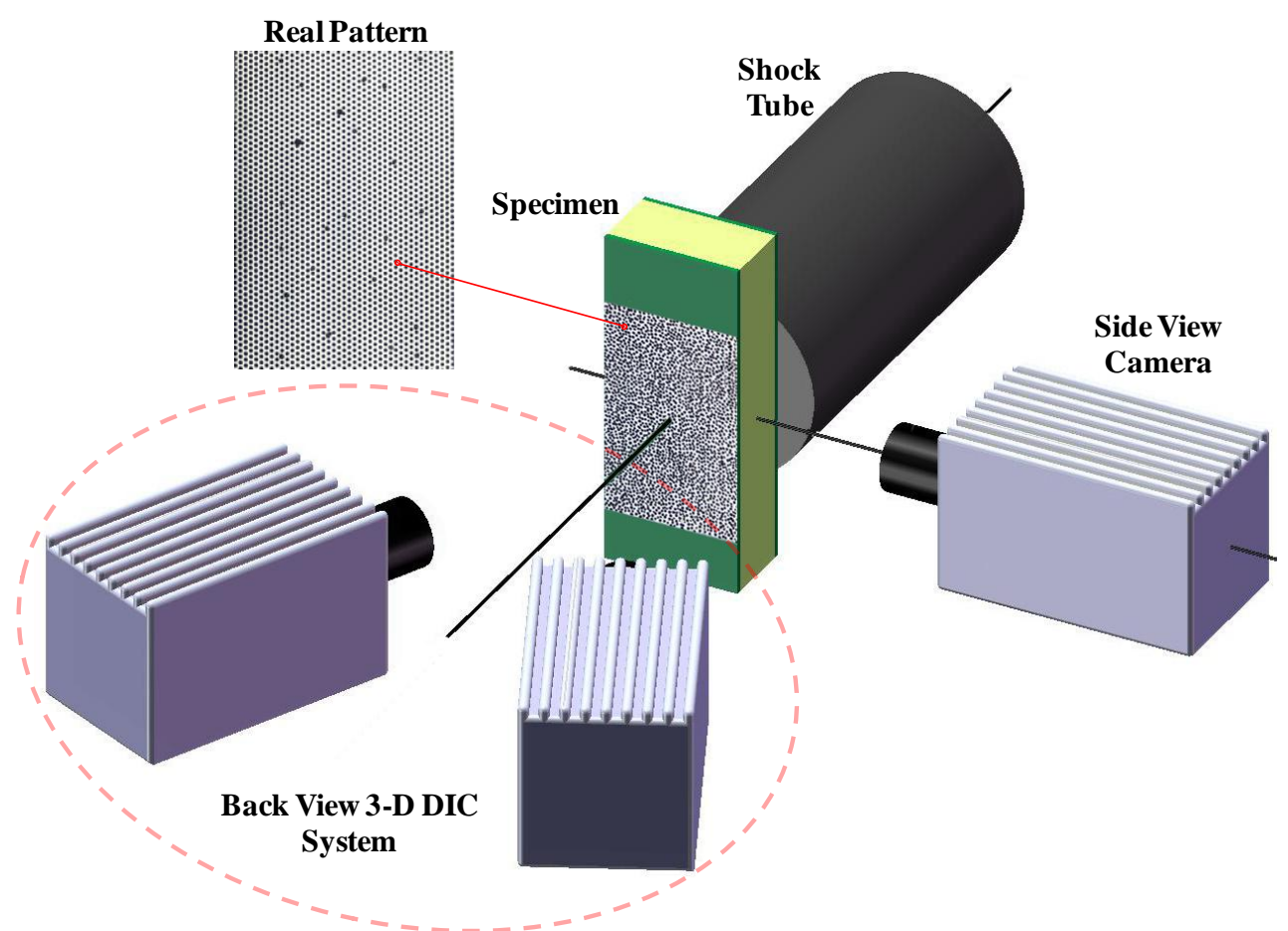

Fig. 6 High-speed photography set-up (Back-view DIC and side-view)

\subsection{Experimental Procedure and Parameters}

An initial series of experiments was conducted for both configurations and three samples were tested for each. This was followed by a second set of experiments, in which two specimens were tested for each configuration. Two different loading conditions were applied. For the first set of experiments, a simply stacked diaphragm of 5 plies of $0.254 \mathrm{~mm}$ mylar sheets with a total thickness of $1.270 \mathrm{~mm}$ was utilized to generate an impulse loading on the specimen with an incident peak pressure of approximately 1.0 $\mathrm{MPa}$, a reflected peak pressure of approximately 5.0 $\mathrm{MPa}$ and a 
wave speed of approximately $1000 \mathrm{~m} / \mathrm{s}$. A typical pressure profile obtained from the transducer closest to the specimen ( $\sim 0.02 \mathrm{~m}$ away) can be seen in Fig. 7. It should be noted that both pressure transducers were utilized to obtain the shock wave history, i.e. incident / reflected pressure and incident / reflected velocity. However, only the pressure transducer closest to the specimen was used to obtain the pressure applied on the specimen.

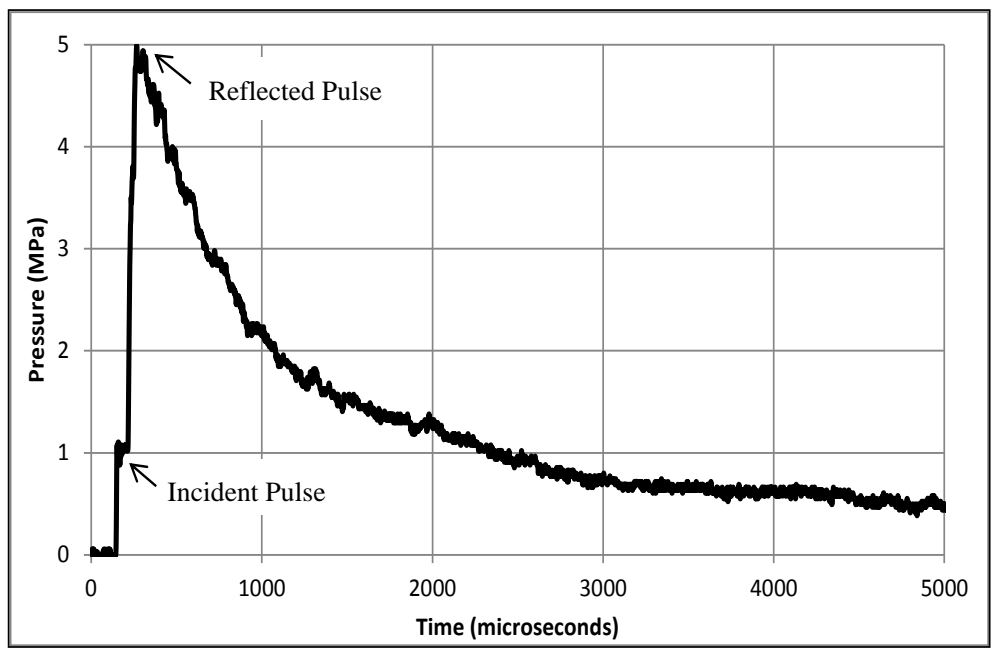

Fig. 7 Incident and reflected pressure profile

For the second set of experiments, a simply stacked diaphragm of 10 plies of $0.254 \mathrm{~mm}$ mylar sheets with a total thickness of $2.540 \mathrm{~mm}$ was utilized to generate an impulse loading on the specimen with an incident peak pressure of approximately 1.5 $\mathrm{MPa}$, a reflected peak pressure of approximately $7.5 \mathrm{MPa}$, and a wave speed of approximately $1300 \mathrm{~m} / \mathrm{s}$. Due to the authors previous work [6], the experiments corresponding to the loading conditions with an incident peak pressure of 1.0 $\mathrm{MPa}$ will be presented and discussed in detail, while the experiments corresponding to the higher loading conditions (incident peak pressure 1.5 MPa) will be utilized to better evaluate the performance and failure mechanisms of the sandwich structures. 


\section{Experimental Results and Discussion}

\subsection{Dynamic Behavior of Core Material}

The quasi-static and dynamic stress-strain curves for the core materials are obtained and shown in Fig.8. The four types of core materials used in the present study have different quasi-static and dynamic behavior. For the same type of Corecell ${ }^{\mathrm{TM}} \mathrm{A}$ foam and Dragonshield-HT polyurea, the material behavior under high strain rate loading is significantly different from its behavior under quasi-static loading.

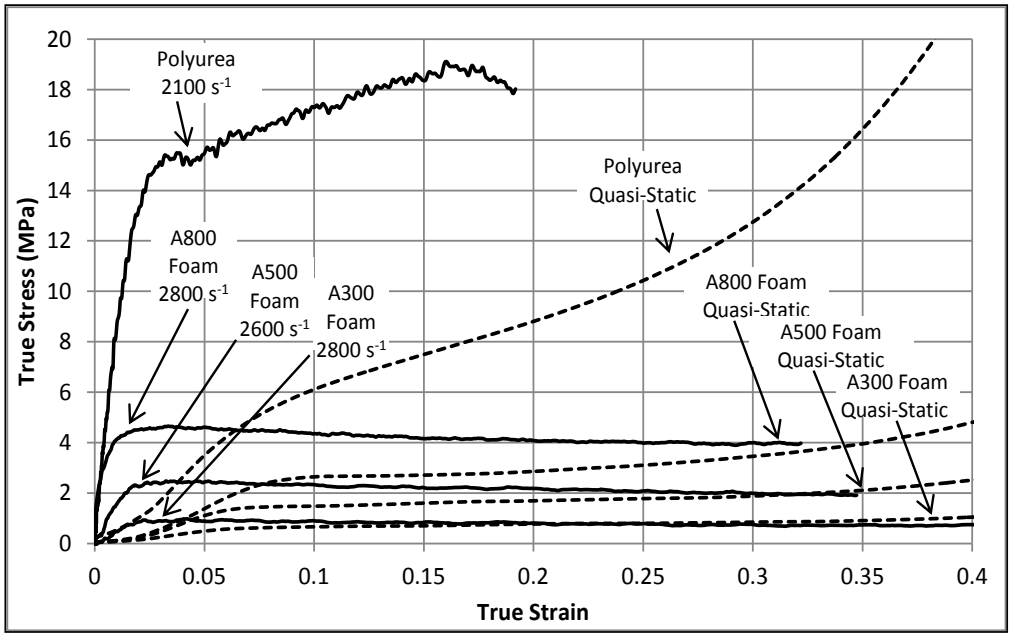

Fig. 8 Quasi-static and high strain-rate compressive behaviors of the different types of core materials

The yield stresses of core materials under quasi-static and high strain rate loading are listed in Table 2. The dynamic yield stress of A500 and A800 increases approximately $100 \%$ in comparison to their quasi-static yield stress, while the dynamic yield stress of A300 increases approximately 50\% in comparison to its quasistatic yield stress. Also it can be observed that the high strain-rate yield stress of Dragonshield-HT polyurea increases approximately $200 \%$ in comparison to its quasistatic yield stress. The improvement of the mechanical behavior from quasi-static to 
high strain-rates for all core materials used in the present study signifies their ability to absorb more energy under high strain-rate dynamic loading.

Table 2 Yield strength of core materials

\begin{tabular}{ccccc}
\hline Foam Type & A300 & A500 & A800 & Polyurea \\
\hline $\begin{array}{c}\text { Quasi-Static } \\
\text { (MPa) }\end{array}$ & 0.60 & 1.35 & 2.46 & 5.38 \\
$\begin{array}{c}\text { High Strain- } \\
\text { Rate (MPa) }\end{array}$ & 0.91 & 2.47 & 4.62 & 15.48 \\
\hline
\end{tabular}

\subsection{Blast Response of Sandwich Composites with Graded Core and PU Interlayer}

\subsubsection{Real-time Deformation}

The real-time observations of the transient behavior for both core configurations subjected to shock wave loading are shown in Fig. 9 and Fig. 10. The shock wave propagates from the right side of the image to the left side and some detailed deformation and failure mechanisms are pointed out in the figures. The time frames used to represent the blast loading event are chosen in a manner so they can be correlated to the time in which these damage mechanisms were first observed.

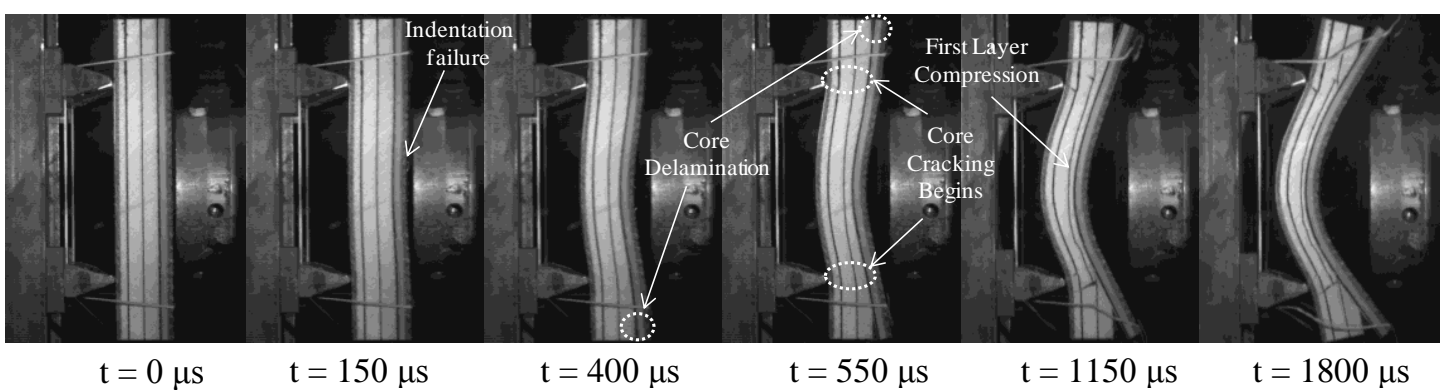

Fig. 9 High-speed images for configuration 1 (PU/A300/A500/A800)

The real-time blast loading response of configuration 1 (PU/A300/A500/A800) is shown in Fig. 9. It can be observed from the images that indentation failure (initiation 
of core compression) begins at approximately $\mathrm{t}=150 \mu \mathrm{s}$. Following indentation failure, core delamination is first observed at $\mathrm{t}=400 \mu \mathrm{s}$, and occurs at the bottom of the specimen between the polyurea interlayer and the first layer of foam core. By $\mathrm{t}=$ $550 \mu$ s more core delamination is observed at the top of the specimen again between the polyurea interlayer and the first layer of foam core. Also at this time two central core cracks have initiated where the supports are located, and heavy core compression is present in the first layer of the foam core $(\mathrm{A} 300)$. By $t=1150 \mu \mathrm{s}$, the first layer of foam core (A300) has reached a maximum level of compression $(8 \mathrm{~mm})$, approximately $75 \%$ of its original thickness $(12.7 \mathrm{~mm})$. After this time, the response is global bending of the specimen and by $\mathrm{t}=1800 \mu \mathrm{s}$, no new failure mechanisms were observed. Also the core cracks have propagated through the foam core to the polyurea interlayer and there is heavy core delamination between the polyurea interlayer and the foam core.

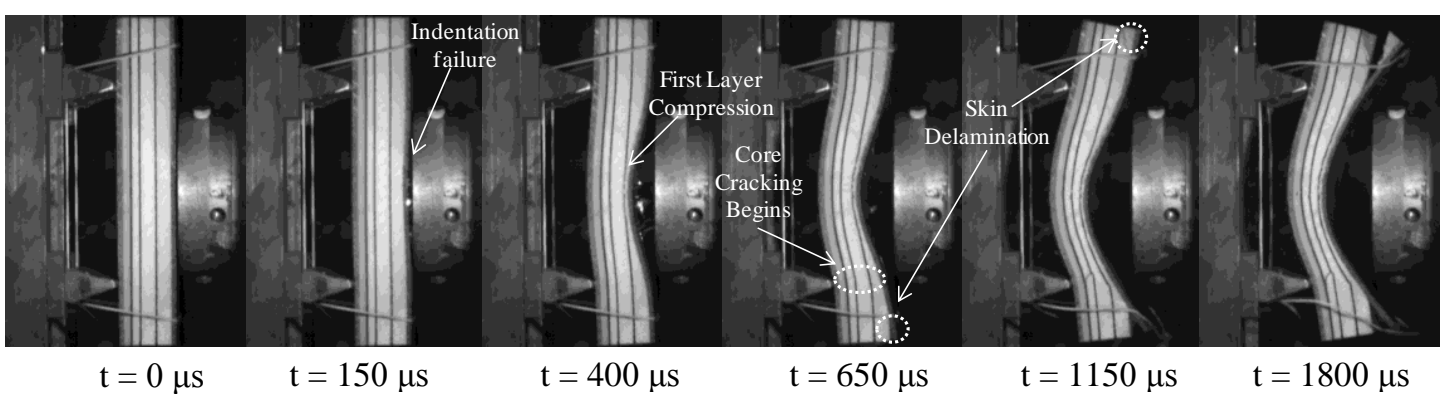

Fig. 10 High-speed images for configuration 2 (A300/A500/A800/PU)

The real-time blast loading response of configuration 2 (A300/A500/A800/PU) is shown in Fig. 10. It is evident from the figure that indentation failure begins at $t=150$ $\mu$ s. After indentation failure is observed, heavy core compression is observed in the first core layer (A300 foam). By $t=650 \mu$ s the first layer of foam (A300) has compressed to a maximum, reaching its densification level, and the shock wave has 
now propagated into the second foam core layer (A500), initiating compression of this core layer. Also at this time, a core crack has initiated at the bottom of the specimen where the support is located. Skin delamination is evident between the front skin and the foam core, and is located at the bottom of the specimen. At $t=1150 \mu$ s skin delamination can be observed at the top of the specimen between the front facesheet and the foam core. Also at this time the compression in the second foam core layer has increased to its maximum and no more compression is observed in the core, resulting in a global bending response. Between $\mathrm{t}=1150 \mu \mathrm{s}$ and $\mathrm{t}=1800 \mu \mathrm{s}$, no new failure mechanisms were observed. The core crack continues to propagate through the third layer of the foam core (A800) and skin delamination at the bottom of the specimen has increased between the front facesheet and the foam core.

Comparing the deformation mechanisms observed in configuration 1 and configuration 2, the location of the polyurea interlayer affects the order and level of different failure mechanisms, such as core compression, core cracking and interface delamination, as well as the time at which they are first observed. In configuration 1 , indentation failure (core compression) is followed by delamination in the core and then core cracking. Unlike configuration 1, the indentation failure of configuration 2 is followed by heavy core compression, core cracking and then skin delamination. Comparing both configurations, the indentation failure is observed at approximately the same time, while core cracking and delamination initiate in configuration 1 earlier in the deformation, approximately $100 \mu \mathrm{s}$ and $250 \mu \mathrm{s}$ respectively, than in configuration 2. 
The location of the polyurea interlayer also affects the core deformation mode for each configuration. In configuration 1 the initial blast loading is uniformly distributed over the polyurea layer, resulting in a global uniform compression of the first layer of the foam core (A300). On the contrary, in configuration 2 the initial impulse is nonuniformly distributed into the foam core, resulting in a local compression in the central region of the first layer of the foam core (A300) where the shock loading was applied. This indicates that the polyurea interlayer has the ability to disperse the shock loading.

Also, the deformation shape for both configurations is much different. For configuration 1, the specimen exhibits a double-winged deformation shape until approximately $\mathrm{t}=400-500 \mu \mathrm{s}$, then the polyurea layer begins to delaminate from the core, exhibiting a shape much like a specimen in pure bending. For configuration 2, the specimen exhibits a double-winged deformation shape throughout the entire blast loading event. Therefore, configuration 2 has the ability to support the shear stresses that are present during the event, while configuration 1 could not.

\subsubsection{Deflection}

The mid-point deflections of the constituents of sandwich composites with different core configuration were obtained from the high-speed side-view images and shown in Fig. 11. For configuration 1, the mid-point deflection of the front face (front skin), interface 1 (between first and second core layer), interface 2 (between second and third core layer), interface 3 (between third and fourth core layer), and back face (back skin) were plotted and are shown in Fig. 11a. The front face and interface 1 deflect in the same manner to the same value of approximately $43 \mathrm{~mm}$ at $\mathrm{t}=1800 \mu \mathrm{s}$, while interface 2 , interface 3 , and the back face deflect in a similar manner to 
approximately $34 \mathrm{~mm}$. Since the front face and interface 1 deflect in the same manner to the same value $(43 \mathrm{~mm})$, it signifies that the polyurea interlayer, which is located between the front face and interface 1, exhibits no compression. The difference between the deflection of interface 1 and interface 2 indicates the total amount of compression observed in the second core layer, which is the first layer of foam (A300). It can be seen that the A300 layer of foam compressed approximately $9 \mathrm{~mm}$, which is $75 \%$ of its original thickness $(12.7 \mathrm{~mm})$. Since interface 2, interface 3 , and the back face all deflected in a similar manner to the same value of approximately 34 $\mathrm{mm}$, it can be concluded that the A500 foam layer (located between interface 2 and interface 3) and the A800 foam layer (located between interface 3 and the back face) showed no compression. Therefore, the core layer arrangement of configuration 1 allows for compression only in the A300 layer of foam and has a front face and back face deflection of approximately $43 \mathrm{~mm}$ and $34 \mathrm{~mm}$ respectively.
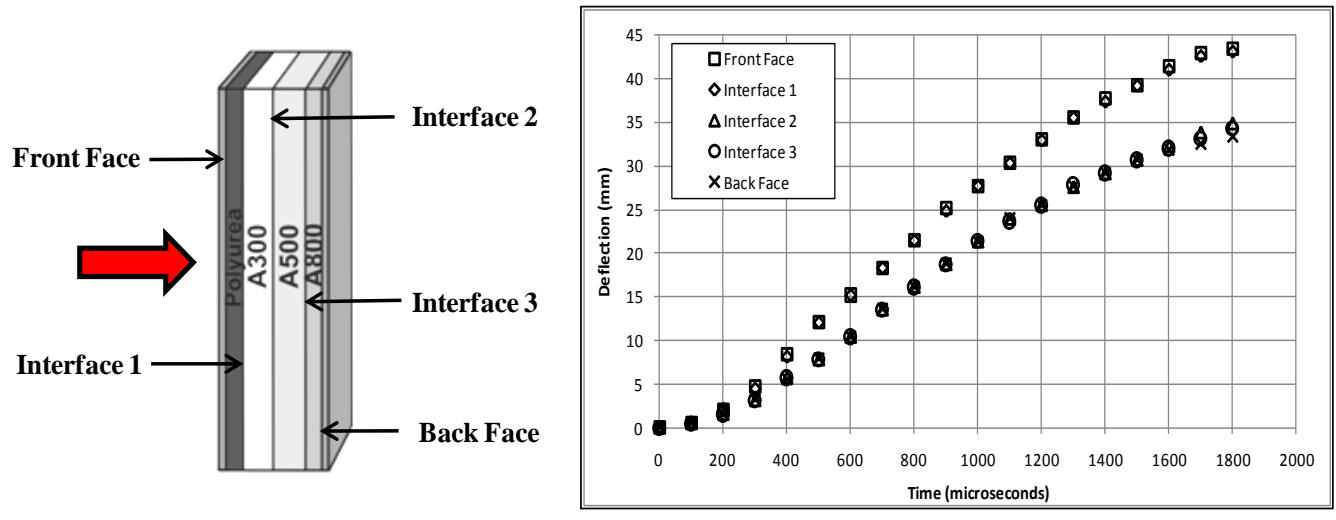

(a) Configuration 1 (PU/A300/A500/A800) 

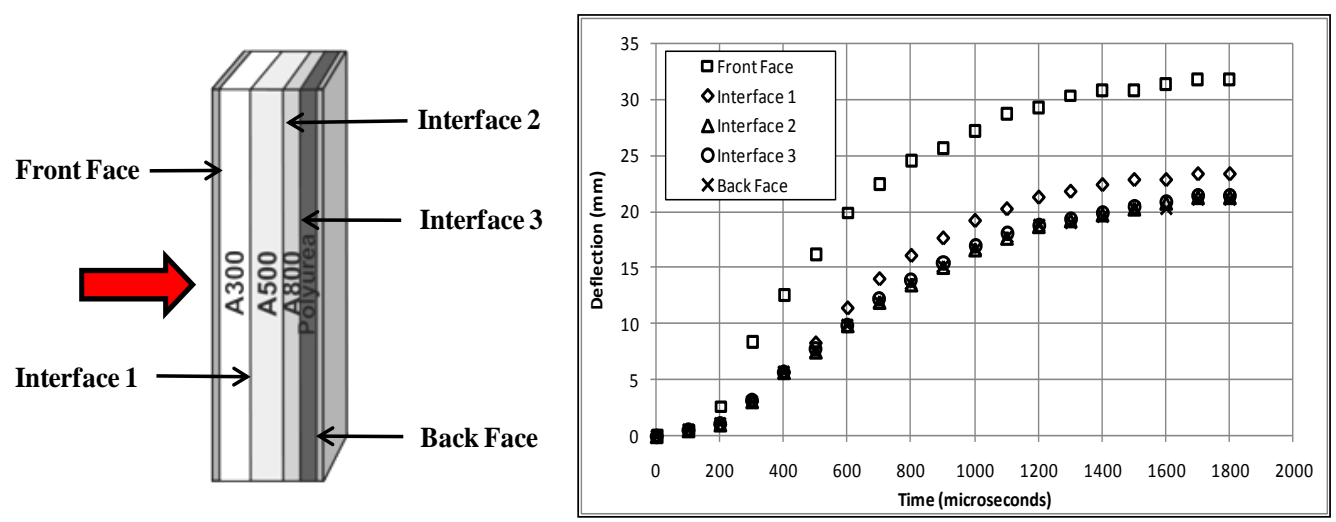

(b) Configuration 2 (A300/A500/A800/PU)

Fig. 11 Mid-point deflection of both configurations

For configuration 2, the mid-point deflection of the front face (front skin), interface 1 (between first and second core layer), interface 2 (between second and third core layer), interface 3 (between third and fourth core layer), and back face (back skin) were plotted and are shown in Fig. 11b. The front face deflected to approximately $33 \mathrm{~mm} \mathrm{t}=1800 \mu \mathrm{s}$, while interface 1 deflected to approximately 24 $\mathrm{mm}$, and interface 2 , interface 3 and the back face deflected in the same manner to a value of $21 \mathrm{~mm}$ respectively. The difference between the front face deflection and the deflection of interface 1 signifies the amount of compression in the first core layer (A300 foam). Therefore, it can be observed that the A300 foam layer compressed approximately $9 \mathrm{~mm}$, or $75 \%$ of its original thickness $(12.7 \mathrm{~mm})$. Again, noting the difference between the deflection of interface 1 and interface 2 the amount of compression in the second core layer (A500 foam) can be obtained. By inspection the A500 foam core layer compresses approximately $3 \mathrm{~mm}$, which is approximately $25 \%$ of its original thickness. Finally, since interface 2, interface 3 and the back face all deflected in a similar manner to the same value of approximately $21 \mathrm{~mm}$, it can be concluded that there was no compression in the third and fourth core layer (A500 
foam layer and the polyurea interlayer). Therefore, the core arrangement of configuration 2 allows for a stepwise compression through the core and the front face and back face deflect to approximately $33 \mathrm{~mm}$ and $21 \mathrm{~mm}$ respectively.

From the deflection data of each interface in Fig. 11, the deformation of each core layer along the mid- line (line of symmetry) can be obtained by subtracting the core layers' back side deflection from the core layers' front side deflection. Sequentially, the strain rate along the line of symmetry of each core layer can be obtained using the following equation,

$$
\begin{gathered}
\text { strain }=\varepsilon=\frac{(\Delta \mathrm{l})}{\mathrm{l}_{\text {original }}} \\
\text { strain rate }=\frac{\mathrm{d} \varepsilon}{\mathrm{dt}}=\frac{\mathrm{d}}{\mathrm{dt}}\left(\frac{(\Delta \mathrm{l})}{\mathrm{l}_{\text {original }}}\right)=\frac{1}{\mathrm{l}_{\text {orignal }}}\left(\frac{\mathrm{d}(\Delta \mathrm{l})}{\mathrm{dt}}\right)
\end{gathered}
$$

where, $l_{\text {original }}$ is the original thickness of the each core layer and $\Delta l / d t$ is the deformation rate.

The strain and strain rate histories of the core layers for each configuration, as calculated from Eq. (1) and Eq. (2) using the mid-point deflection data from Fig.11 are shown in Fig. 12. For those layers exhibiting no compression, their strain and strain rate results $\left(0 \mathrm{~s}^{-1}\right)$ are not shown here. The strain results are shown in Fig. 12a and Fig. 12b, while the strain rate results are shown in Fig. 12c and Fig. 12d.

It can be seen that in both configurations, the A300 foam layer exhibits approximately the same amount of maximum strain, however the time in which the maximum strain was reached varied. For configuration 1, distributing the initial loading uniformly results in densification of the A300 foam much later in the deformation history, more mitigation of the initial shock loading and thus less transmission of the load to the A500 layer, resulting in no compression in this layer. 


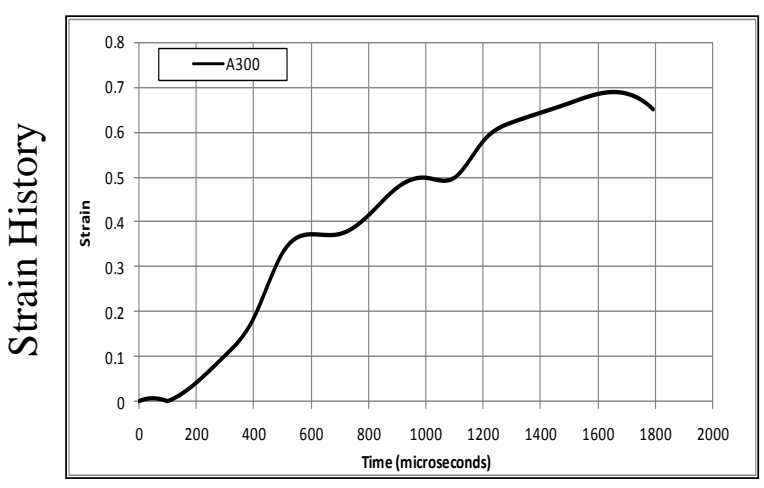

(a) Configuration 1

(PU/A300/A500/A800)

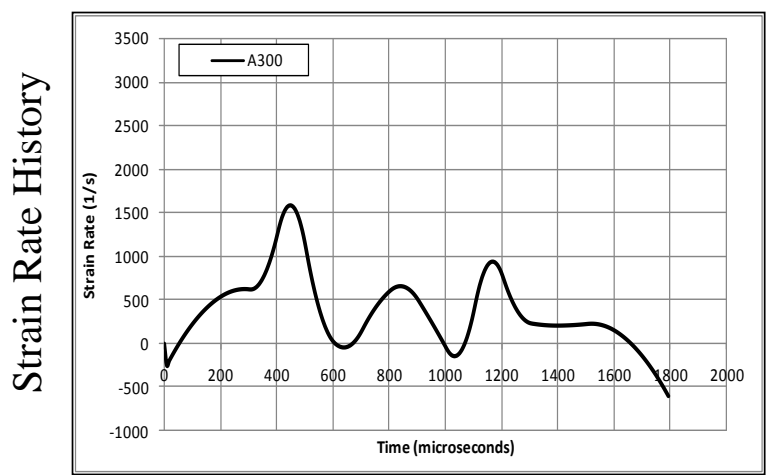

(c) Configuration 1 (PU/A300/A500/A800)

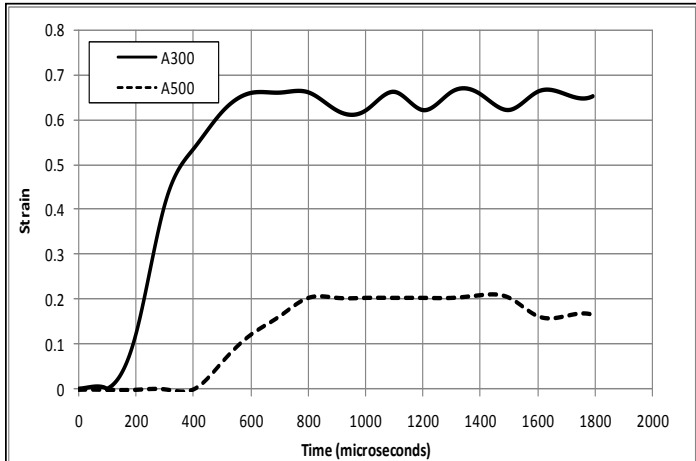

(b) Configuration 2 (A300/A500/A800/PU)

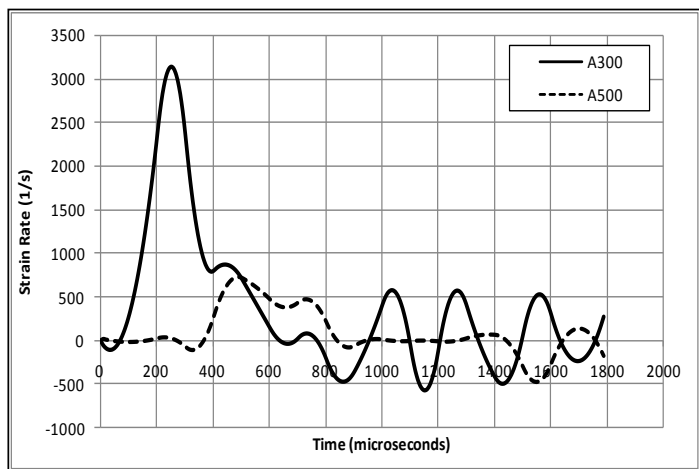

(d) Configuration 2 (A300/A500/A800/PU)

Fig. 12 Strain and strain rate history of each core layer along the mid-line

For configuration 2, unlike configuration 1, localized loading allowed for densification of the A300 foam layer much earlier in the deformation history, and consequently transmitted more shock loading into the A500 layer. Therefore, the A500 layer also showed compression. However, the deformation in configuration 2 is constrained to the central region where the initial loading was applied.

The strain rate plots of configuration 1 and configuration 2 show that the A300 layer of configuration 2 reached a much higher strain rate than the A300 layer of configuration, which was expected since the maximum level of strain in configuration 2 was achieved approximately twice as fast $(\sim 800 \mu$ s earlier $)$ than in configuration 1 . It 
should be noted that in configuration 2 the core stops compressing by approximately $\mathrm{t}$ $=800 \mu \mathrm{s}$, thus the oscillations that are observed in the strain rate plot after this time can be correlated to the errors in data recording, and consequently neglected.

From the mid-point deflection data in Fig. 11a and Fig. 11b, the average midpoint velocities of the front face and back face for both configurations can be obtained by differentiating the front face and back face deflection with respect to time, and are shown in Fig 13. It can be seen in the figure that in configuration 1, the front face and back face reach a maximum velocity together early in the deformation history, $\sim \mathrm{t}=$ $700 \mu$ s, converging on the same common velocity $(\sim 30 \mathrm{~m} / \mathrm{s})$ and then decelerating together. The front face and back face of configuration 2 reach maximum velocities at different times, $\sim \mathrm{t}=400 \mu \mathrm{s}$ and $\sim \mathrm{t}=600 \mu$ s respectively, and the share a common velocity of $\sim 20 \mathrm{~m} / \mathrm{s}$ much later in the event $(\sim 800 \mu \mathrm{s})$, before decelerating together. This suggests that the back face was beginning to decelerate, while the core was still compressing. Such responses and phenomena have been investigated by Liang et al. [20] and Tilbrook et al. [21]. When the front and back face velocities equalize early in the deformation history, this response is labeled as a hard core type response. In contrast, when the back face begins to decelerate while the core is still compressing, this response is labeled as a soft core type response. Therefore, it can be concluded that configuration 1 exhibits a hard core type response, while configuration 2 exhibits a soft core type response. The authors [20,21] suggested that the optimal performance of sandwich beams is attained for soft core designs, which allows for a reduction in the transmitted impulse during the initial fluid-structure interaction stage. 


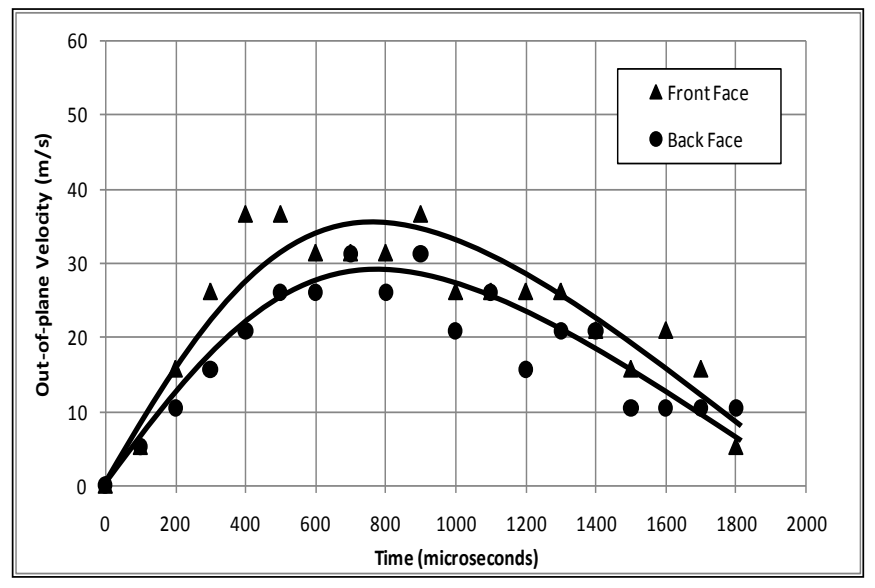

(a) Configuration 1 (PU/A300/A500/A800)

(Hard Core Type Response)

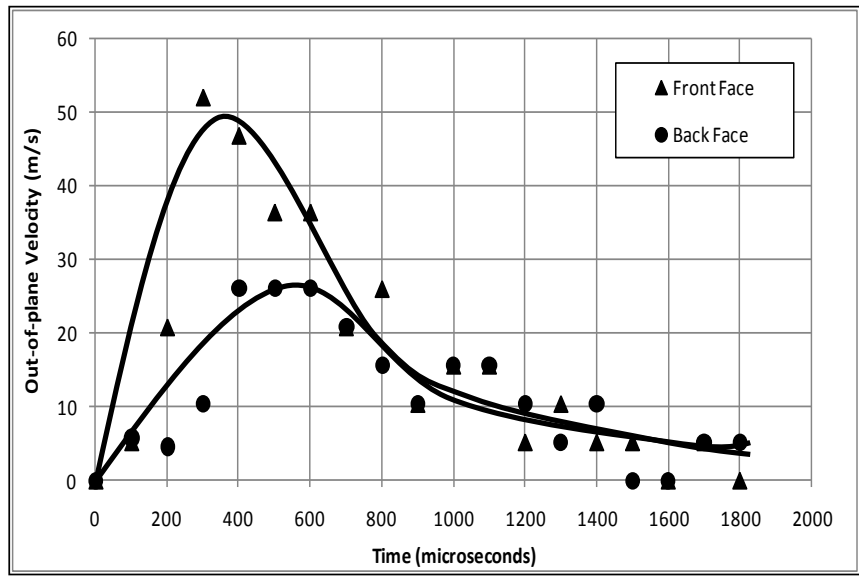

(b) Configuration 2 (A300/A500/A800/PU)

(Soft Core Type Response)

Fig. 13 Front and back face out-of-plane velocities for both configurations

\subsubsection{Digital Image Correlation (DIC) Analysis}

Utilizing the Digital Image Correlation (DIC) technique, the deflection, in-plane strain and particle velocity contours of the back facesheet for each configuration were generated. Fig. 14 - Fig 16 show the full-field results for the back facesheet of configuration 1 and configuration 2 respectively. Fig. 14 shows the full-field out-ofplane deflection (W) with a scale of $0 \mathrm{~mm}$ (purple) to $32 \mathrm{~mm}$ (red). It is evident from the figure that for configuration 1, as shown in Fig. 14a, the back face exhibits very 
little out-of-plane deflection until approximately $\mathrm{t}=400 \mu \mathrm{s}$. Between $\mathrm{t}=400 \mu \mathrm{s}$ and $\mathrm{t}$ $=1800 \mu \mathrm{s}$, the panel continues to show deflection. By $\mathrm{t}=1800 \mu \mathrm{s}$, it can be observed that the central region of the panel has deflected approximately $32 \mathrm{~mm}$. For configuration 2, as shown in Fig. 14b, the back face shows very little out-of-plane deflection until $\mathrm{t}=400 \mu \mathrm{s}$. Between $\mathrm{t}=400 \mu \mathrm{s}$ and $\mathrm{t}=1800 \mu \mathrm{s}$, the panel continues to exhibit deflection. By $\mathrm{t}=1800 \mu \mathrm{s}$, the central region of the panel has deflected approximately $22 \mathrm{~mm}$. Therefore, configuration 2 deflects approximately $35 \%$ less than configuration 1.

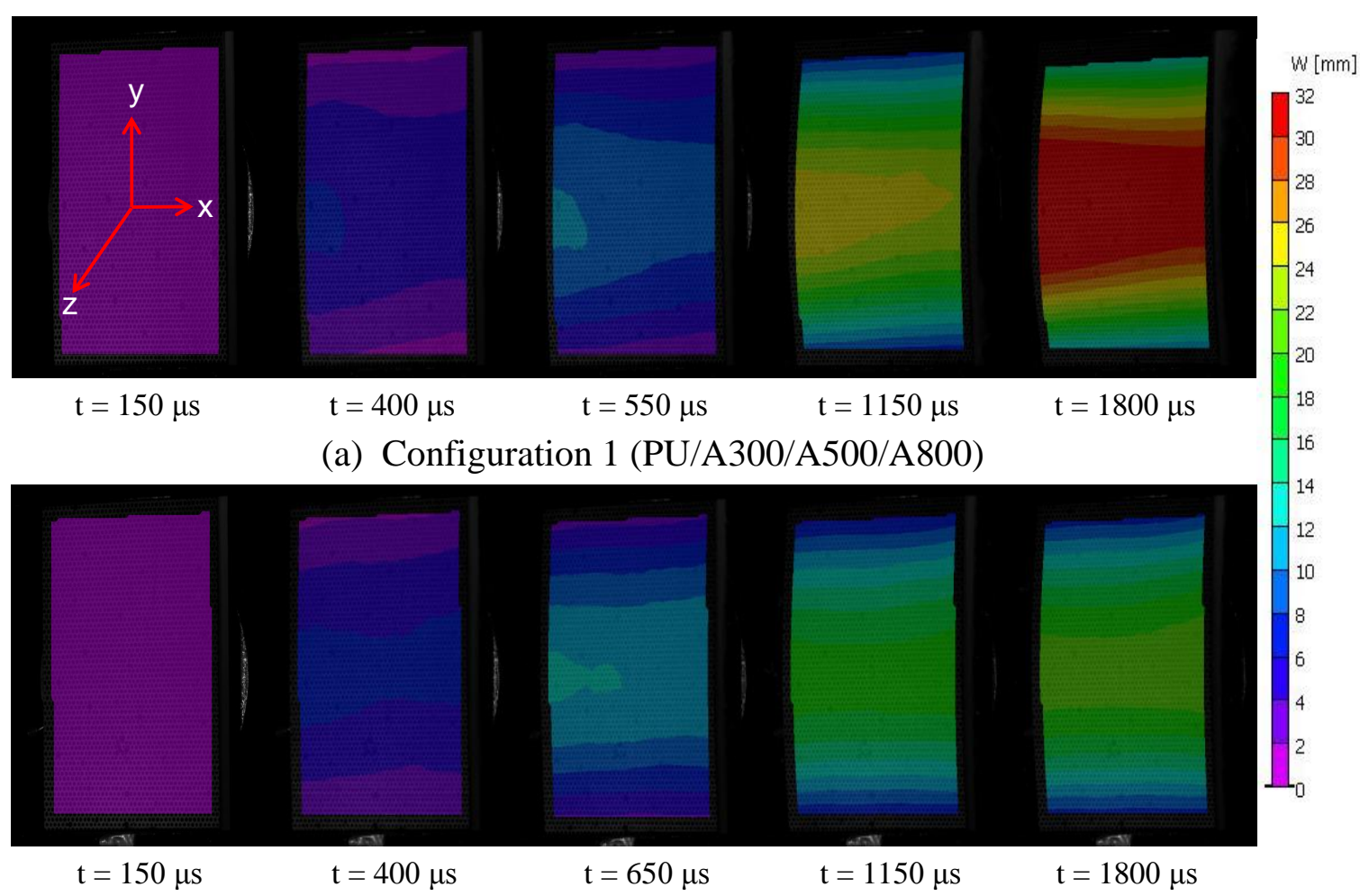

(b) Configuration 2 (A300/A500/A800/PU)

Fig. 14 Full-field out-of-plane deflection (W) of both configurations

Fig. 15 shows the full-field in-plane-strain $\left(\varepsilon_{y y}\right)$ for both configurations with a scale of 0 (red) to 0.026 (purple), or $0 \%$ to $2.6 \%$ respectively. For configuration 1, as shown in Fig. 15a, the back face exhibits very minimal in-plane-strain $\left(\varepsilon_{y y}\right)$ until 
approximately $\mathrm{t}=150 \mu \mathrm{s}$. Between $\mathrm{t}=150 \mu \mathrm{s}$ and $\mathrm{t}=1800 \mu \mathrm{s}$, the in-plane-strain $\left(\varepsilon_{\mathrm{yy}}\right)$ continues to increase. By $\mathrm{t}=1800 \mu \mathrm{s}$, it can be observed that the panel shows a maximum in-plane-strain $\left(\varepsilon_{\mathrm{yy}}\right)$ across the central region of the panel of approximately 2.6\%. For configuration 2, as shown in Fig. 15b, the back face shows very minimal inplane-strain $\left(\varepsilon_{y y}\right)$ until approximately $\mathrm{t}=150 \mu \mathrm{s}$. Between $\mathrm{t}=150 \mu \mathrm{s}$ and $\mathrm{t}=1800 \mu \mathrm{s}$, the in-plane-strain $\left(\varepsilon_{\mathrm{yy}}\right)$ continues to increase. By $\mathrm{t}=1800 \mu \mathrm{s}$, the panel shows a maximum in-plane-strain $\left(\varepsilon_{\mathrm{yy}}\right)$ across the central region of the panel of approximately $1.625 \%$. As a result, configuration 2 exhibits approximately $35 \%$ in-plane-strain than configuration 1 .

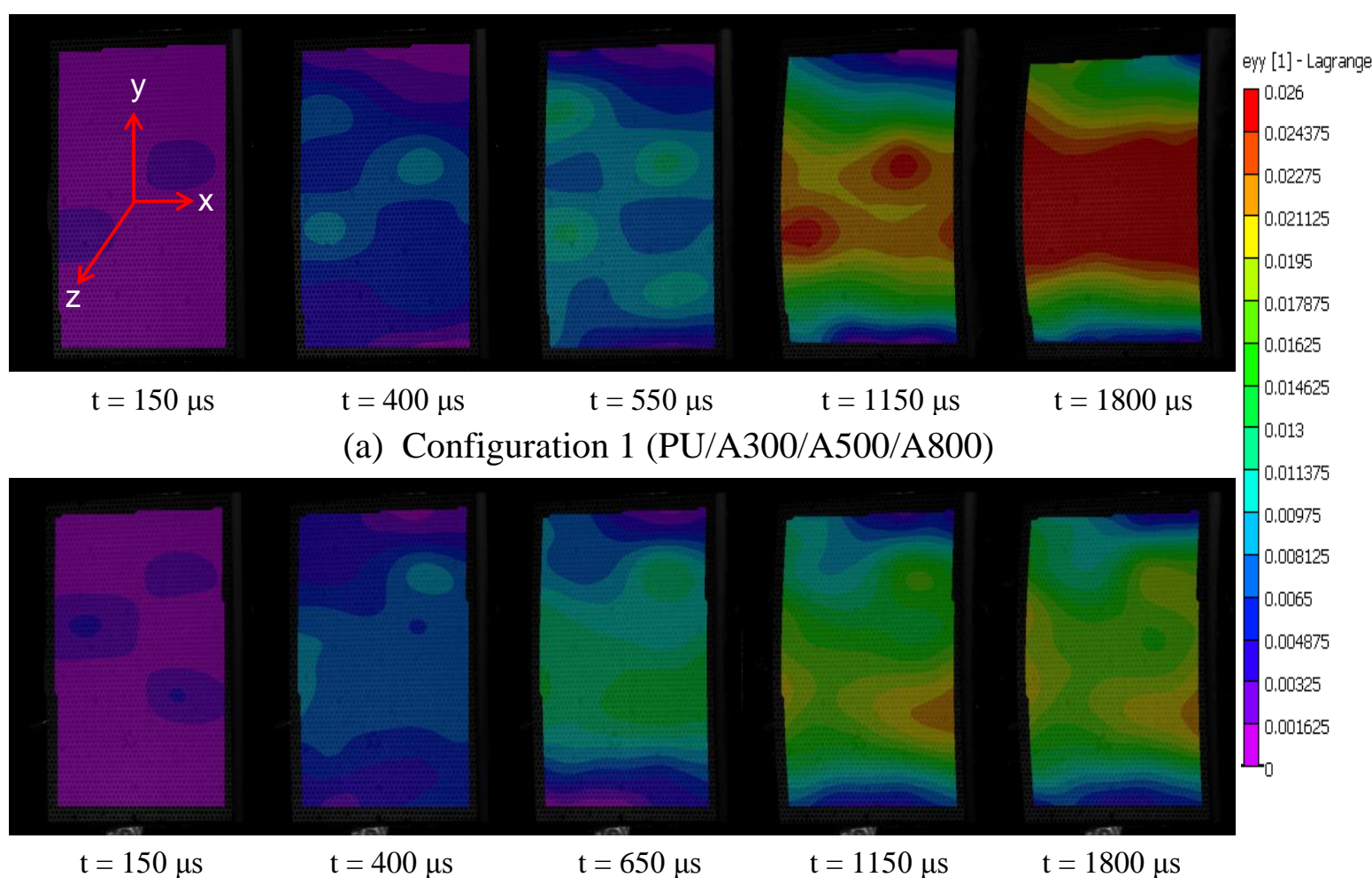

(b) Configuration 2 (A300/A500/A800/PU)

Fig. 15 Full-field in-plane strain $\left(\varepsilon_{\mathrm{yy}}\right)$ of both configurations

The full-field out-of-plane velocity $(\mathrm{dW} / \mathrm{dt})$ of the back face is shown in Fig. 16 for both configurations with a scale from $0 \mathrm{~mm} / \mathrm{s}$ (purple) to $30,000 \mathrm{~mm} / \mathrm{s}$ (red), or 0 
$\mathrm{m} / \mathrm{s}$ to $30 \mathrm{~m} / \mathrm{s}$. For configuration 1 , as shown in Fig. 16a, the back face begins to exhibit out-of-plane velocity by $\mathrm{t}=150 \mu \mathrm{s}$. By $\mathrm{t}=550 \mu \mathrm{s}$, the central region of the back face has reached an out-of-plane velocity of approximately $30 \mathrm{~m} / \mathrm{s}$. By $\mathrm{t}=1150$ $\mu \mathrm{s}$ the velocity has reduced to approximately $22.5 \mathrm{~m} / \mathrm{s}$ and by $\mathrm{t}=1800 \mu \mathrm{s}$ the velocity has reduced to $7.5 \mathrm{~m} / \mathrm{s}$. For configuration 2, as shown in Fig. 16b, the back face begins to begins to show out-of-plane velocity by $\mathrm{t}=150 \mu \mathrm{s}$. By $\mathrm{t}=400 \mu \mathrm{s}$, the central region of the back face has reached an out-of-plane velocity of approximately $24.375 \mathrm{~m} / \mathrm{s}$. From $\mathrm{t}=400 \mu \mathrm{s}$ and onward, the out-of-plane back face velocity continues to be reduced to approximately $22.5 \mathrm{~m} / \mathrm{s}$ at $\mathrm{t}=650 \mu \mathrm{s}$, approximately $7.5 \mathrm{~m} / \mathrm{s}$ at $\mathrm{t}=1150 \mu \mathrm{s}$, and approximately $0 \mathrm{~m} / \mathrm{s}$ by $\mathrm{t}=1800 \mu \mathrm{s}$. Subsequently, configuration 2 reduces the back face velocity by approximately $15 \%$ in comparison to configuration 1 .

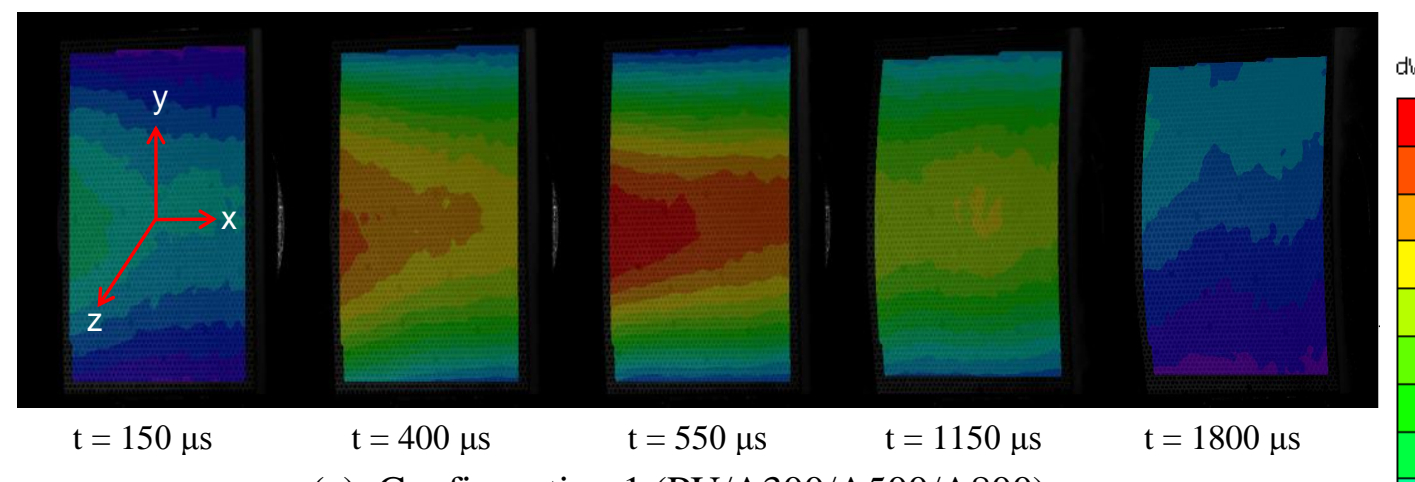
$\mathrm{dW} / \mathrm{dt}[\mathrm{mm} / \mathrm{s}]$ 30000 28125

(a) Configuration 1 (PU/A300/A500/A800)

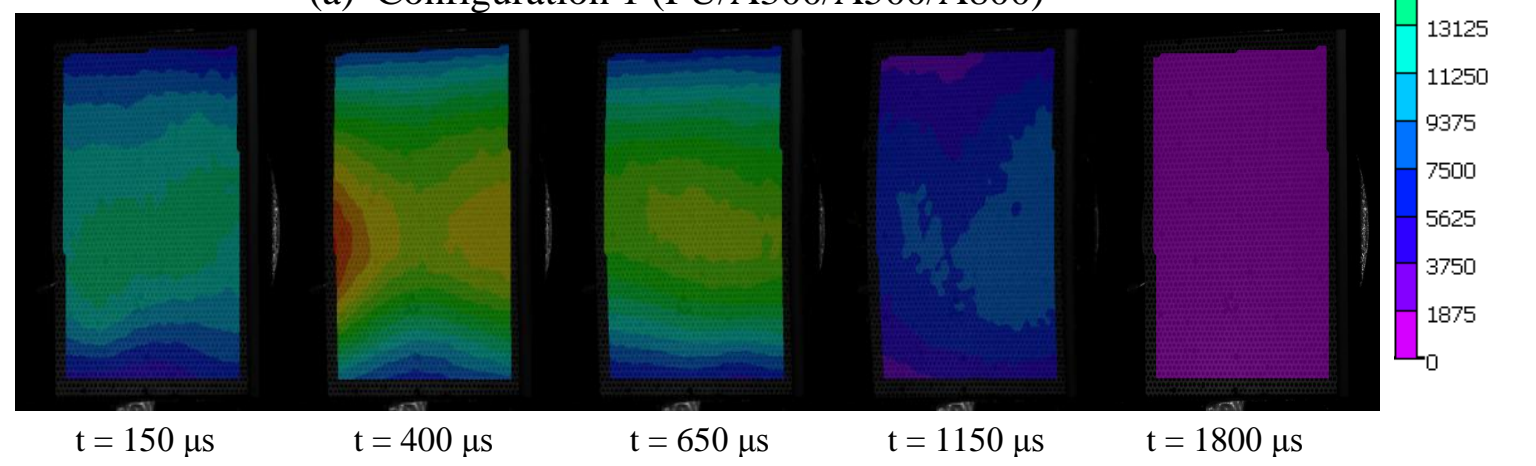

(b) Configuration 2 (A300/A500/A800/PU)

Fig. 16 Full-field out-of-plane velocity $(\mathrm{dW} / \mathrm{dt})$ of both configurations 
Utilizing a point-inspection tool from digital image correlation, the data at the center point of the back facesheet for each configuration was evaluated and plotted. The out-of plane deflection, as well as the back face velocity, showed excellent agreement with the results generated utilizing the side-view high-speed images, and therefore only the in-plane strain results are shown in Fig. 17. The back face of configuration 1 exhibits a maximum in-plane strain $\left(\varepsilon_{y y}\right)$ of approximately $2.4 \%$ at $\mathrm{t}=$ $1800 \mu$ s. For configuration 2 , the back face shows a maximum in-plane- strain $\left(\varepsilon_{y y}\right)$ of approximately $1.6 \%$ at $\mathrm{t}=1800 \mu$ s. Configuration 2 exhibits approximately $35 \%$ less in-plane-strain $\left(\varepsilon_{\mathrm{yy}}\right)$ than configuration 1.

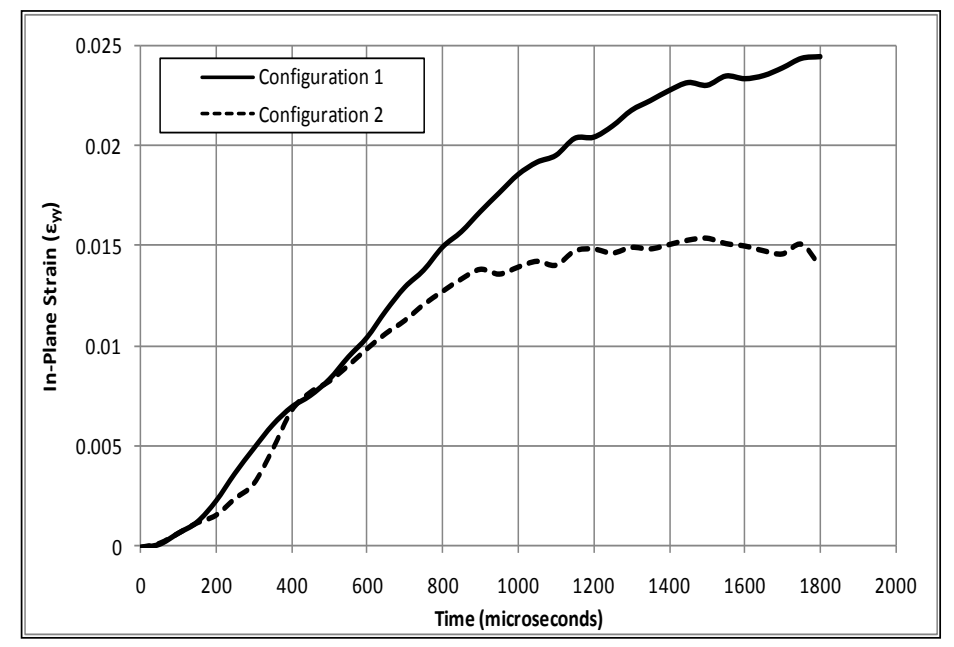

Fig. 17 In-plane strain $\left(\varepsilon_{y y}\right)$ for both configurations

\subsubsection{Post-mortem Analysis}

After the blast loading event occurred, the damage patterns of both configuration 1 and configuration 2 were visually examined and recorded using a high resolution digital camera and are shown in Fig. 18. When configuration 1 was subjected to transient shock wave loading, as shown in Fig. 18a, the damage was confined to the areas where the supports were located in the shock tube and core cracking is visible in 
these two areas. The core cracks propagated completely through the foam core to the polyurea interlayer. Core delamination is visible between the polyurea interlayer, and the first layer of the foam core (A300). Core compression is visible in the first core layer of A300 foam.

When configuration 2 was subjected to transient shock wave loading, the damage patterns can be seen in Fig. 18b. For this configuration, very little core damage was observed. Core delamination between the first two layers of the foam core (A300 and A500) led to a crack that propagated through the first foam core layer (A300) to the front facesheet. Skin delamination was evident between the front face and the first foam core layer (A300). Also core compression can be observed in the first two layers of the foam core (A300 and A500).

Front facesheet (Blast-receiving side)

\section{Foam and Polyurea Core}

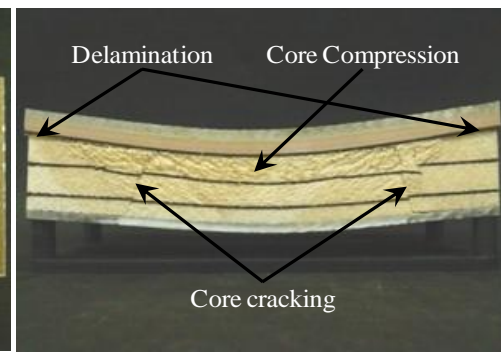

(a) Configuration 1 (PU/A300/A500/A800)
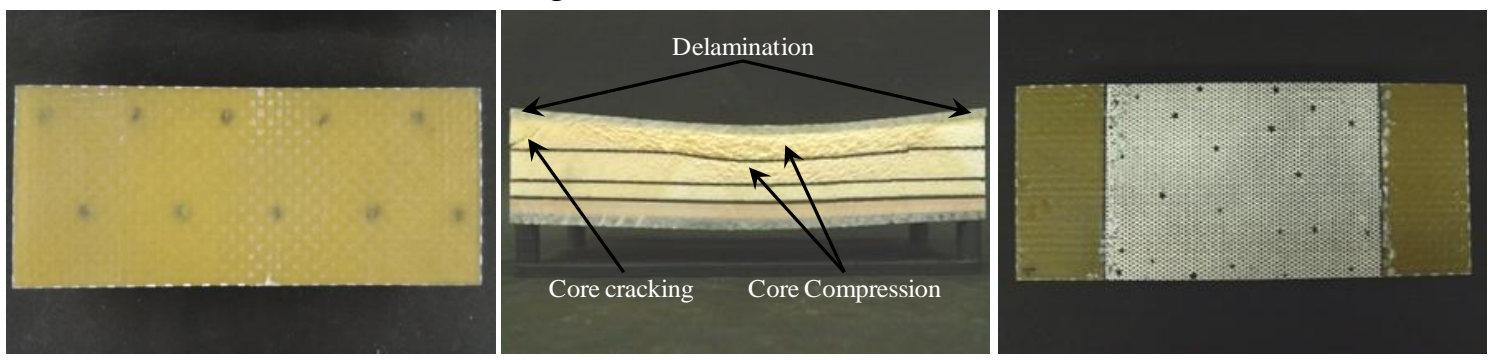

(b) Configuration 2 (A300/A500/A800/PU)

Fig. 18 Visual examination of both configurations after being subjected to high intensity blast load (Incident peak pressure $\sim 1.0 \mathrm{MPa}$ ) 
Fig. 19 shows the damage patterns of both configuration 1 and configuration 2 after they were subjected to the higher levels of blast loading (incident peak pressure 1.5 MPa, reflected peak pressure 7.5 MPa, wave velocity of $1300 \mathrm{~m} / \mathrm{s}$ ). It can be seen from the figure that when configuration 1, as seen in Fig. 19a, was subjected to a higher level of blast loading, the core exhibited heavy core cracking which lead to catastrophic failure. The font face showed heavy fiber delamination and cracking across the central region, while the back face delaminated completely from the core.

Front facesheet (Blast-receiving side)

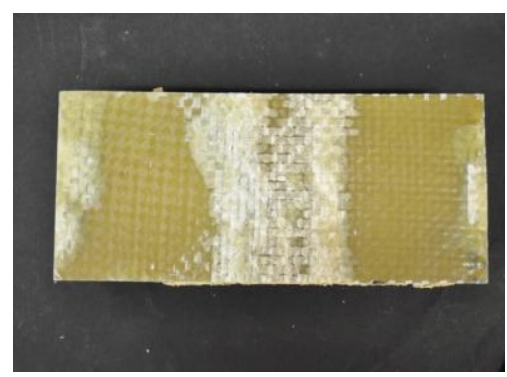

Foam and Polyurea Core

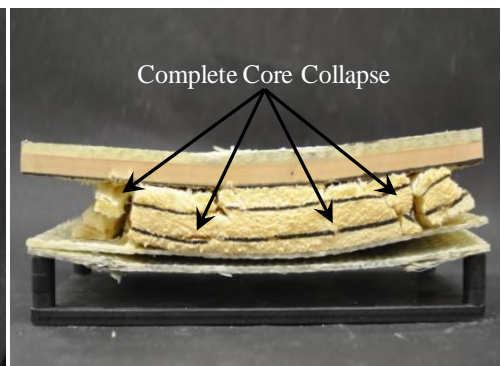

(a) Configuration 1 (PU/A300/A500/A800)
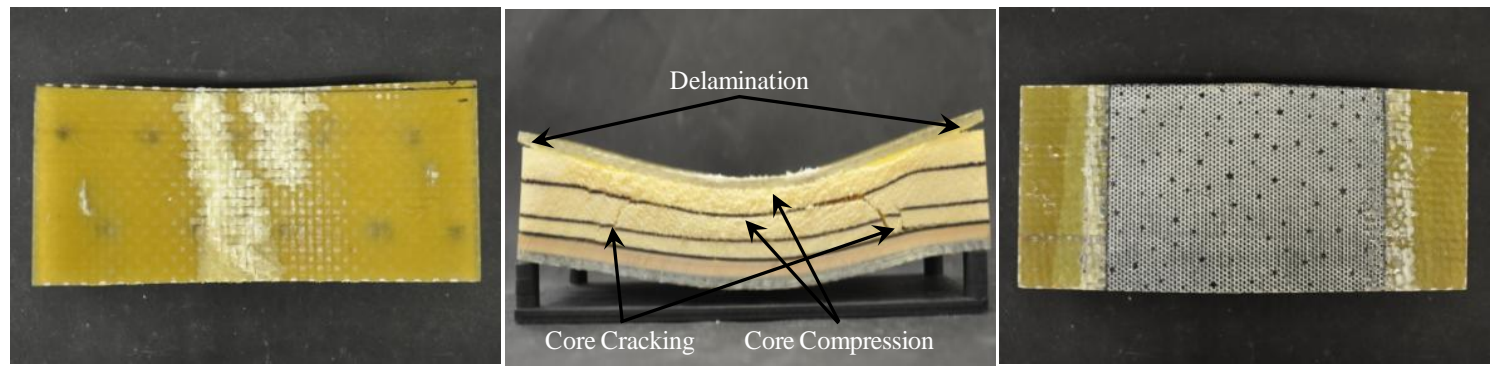

(b) Configuration 2 (A300/A500/A800/PU)

Fig. 19 Visual examination of both configurations after being subjected to high intensity blast load (Incident peak pressure $\sim 1.5 \mathrm{MPa}$ )

Configuration 2 on the other hand, as seen in Fig. 19b, remained structurally intact after the higher level of blast loading. The front face showed minor fiber delamination, while the core exhibited cracking along the central region where the supports were located. Minor front skin delamination was evident between the front 
face and the first foam core layer (A300). Also core compression can be observed in the first and second core layers of foam, A300 and A500 respectively.

\subsubsection{Energy Redistribution Behavior}

The energy redistribution behavior of both configurations was next analyzed using the methods described by Wang et al. [22]. The total energy loss and the total deformation energy of both configuration 1 and configuration 2 during the blast loading event are shown in Fig. 20 and Fig. 21 respectively. Total energy loss is characterized as the difference between the incident and remaining energies of the gas and total deformation energy is defined as the work done by the gas to deform the specimen. It can be observed in Fig. 20 and Fig. 21 that at $\mathrm{t}=1800 \mu$ s, the total energy loss of configuration 1, as well as the deformation energy, is approximately $25 \%$ more than that of configuration 2 . This indicates that configuration 1 has the ability to consume more energy during the shock loading process, as discussed in Section 4.2.1, but results in heavier core damage as observed in Fig. 18a and Fig. 19a. This phenomenon is directly related to the location of the polyurea layer and has been described by Amini et al. [13-16]. Polyurea is a highly pressure sensitive elastomer with its shear and bulk stiffness increasing remarkably with increasing pressure [8]. When polyurea is applied to the front of the specimen, behind the facesheet and in front of the foam core, the confined polyurea is loaded in compression, increasing its bulk stiffness and thus attaining a better impedance match with the facesheet. Consequently, more of the blast energy is transferred to the foam core.

On the contrary, when polyurea is applied to the back of the specimen, behind the foam core and in front of the facesheet, the foam core is loaded first and then a part of 
this energy is transferred to the polyurea. This compresses the polyurea layer, thus increasing its stiffness, and therefore increasing the amount of energy that it captures. This behavior can be elucidated by the fact that as the pressure-pulse travels through the polyurea layer and subsequently through the back facesheet, it is reflected back off its free-face surface as a tensile release wave. This results in a substantial decrease in the polyurea's shear stiffness and concurrently substantial increase in its dissipative ability due to its viscoelasticity. This phenomenon can be observed in the overall behavior of configuration 2. From the high-speed images in Fig. 10, it can be seen that the foam core is loaded first, resulting in heavy core compression as discussed in Section 4.2.1 and shown in Fig. 18b and Fig 19b. In comparison to configuration 1, the peak values of back face deflection, strain and velocity are reduced by $35 \%, 35 \%$ and $15 \%$ respectively, as shown in Fig. $11 \mathrm{~b}, 13 \mathrm{~b}$ and 17 . This means the energy that is transferred to the back face of configuration 2 is less than that of configuration 1.

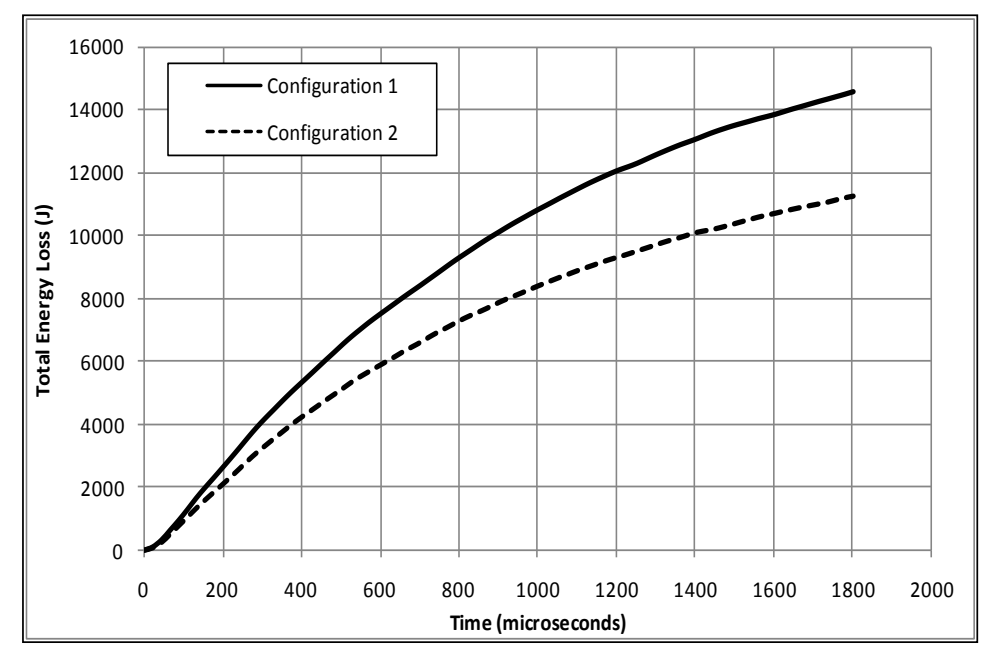

Fig. 20 Total energy loss in both configurations during blast loading event 


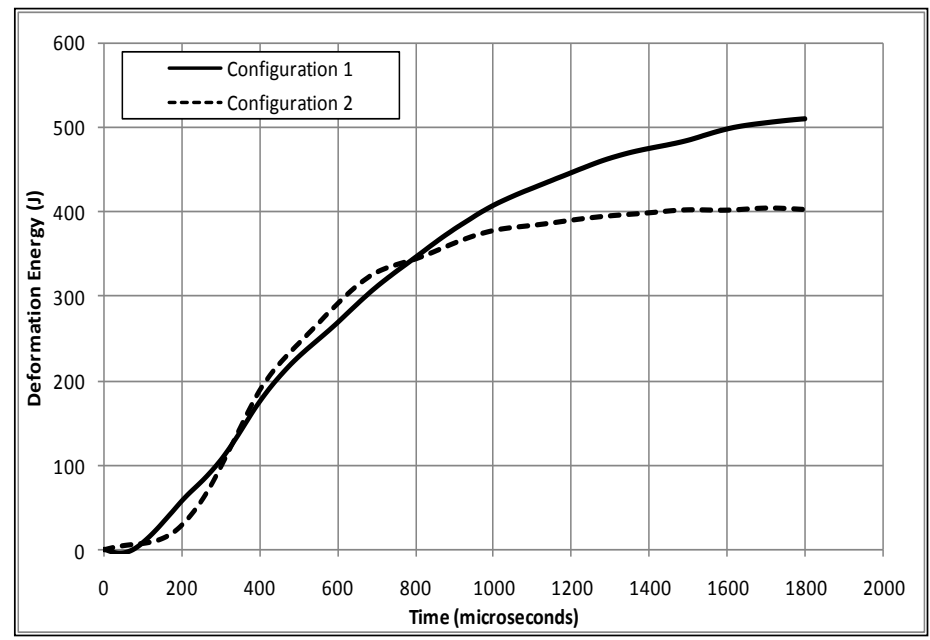

Fig. 21 Deformation energy of both configurations during blast loading event

Therefore, it can be concluded that the location of the polyurea layer has a significant effect on the response of composite sandwich panels to shock wave loading, both in terms of failure mitigation and energy absorption, if it is placed opposite the blast-receiving side (configuration 2). On the contrary, the presence of polyurea on the blast-receiving side (configuration 1), amplifies the destructive effect of the blast, promoting (rather than mitigating) the failure of the composite sandwich panels.

\section{Conclusions}

The following is the summary of the investigation:

(1) The dynamic stress-strain response is significantly higher than the quasi-static response for each type of core material used in the present study, Corecell ${ }^{\mathrm{TM}}$ A-series foam and Dragonshield-HT polyurea respectively. The quasi-static and dynamic constitutive behaviors of Corecell $^{\mathrm{TM}}$ A series foams (A300, A500, and A800) as well as the polyurea interlayer show an increasing trend. The increase in the yield strength from quasi-static response to dynamic 
response, along with the longer stress plateau, indicates that these core materials show great potential in absorbing large amounts of energy.

(2) Sandwich composites with two types of core layer arrangements were subjected to shock wave loading. Both core configurations consisted of three (3) types of Corecell ${ }^{\mathrm{TM}}$ foam and a polyurea (Dragonshield - HT) interlayer. The foam core was monotonically graded based on increasing wave impedance, and the only difference between the two core configurations arose in the location of the polyurea interlayer. It was observed that when the polyurea interlayer is located behind the graded foam core, and in front of the back face (i.e. configuration 2), the core layer arrangement allows for a stepwise compression of the core. Larger compression was visible in the A300 and A500 foam core layers of configuration 2 than configuration 1 . This compression lowers the strength of the initial shock wave by the time it reaches the back facesheet and thus the overall deflection, in-plane strain, and velocity were reduced in comparison to the sandwich composite with the polyurea interlayer located behind the front facesheet and in front of the foam core (i.e. configuration 1). Therefore, it can be concluded that placing the polyurea interlayer behind the foam core and in front of the back facesheet (configuration 2) improves the blast resistance of the sandwich composite and better maintains structural integrity.

(3) Comparison of the mid-point deflection of both configurations was made using high-speed photography (side-view images) and the Digital Image Correlation 
(DIC) technique. Results obtained using both methods of analysis showed excellent agreement with a small margin of error $(<5 \%)$.

(4) The methods used to evaluate the energy as described by Wang et al. [22] were implemented and the results analyzed. It was observed that the location of the polyurea layer has a significant positive effect on the response of composite sandwich panels to shock wave loading, both in terms of failure mitigation and energy absorption, if it is placed opposite the blast-receiving side (configuration 2). On the contrary, the presence of polyurea on the blastreceiving side (configuration 1), amplifies the destructive effect of the blast, promoting (rather than mitigating) the failure of the composite sandwich panels.

\section{Acknowledgements}

The authors kindly acknowledge the financial support provided by Dr. Yapa D. S. Rajapakse, under Office of Naval Research Grant No. N00014-04-1-0268. The authors acknowledge the support provided by the Department of Homeland Security under Cooperative Agreement No. 2008-ST-061-ED0002. Authors thank Gurit SP Technology and Specialty Products Incorporated (SPI) for providing the material as well as Dr. Stephen Nolet and TPI Composites for providing the facility for creating the composites used in this study.

\section{References}

[1] Xue Z, Hutchinson JW (2003) Preliminary assessment of sandwich plates subject to blast loads. Int J Mech Sci 45: 687-705. 
[2] Fleck NA, Deshpande VS (2004) The resistance of clamped sandwich beams to shock loading. J App Mech 71: 386-401.

[3] Dharmasena KP, Wadley HNG, Xue Z, Hutchinson JW (2008) Mechanical response of metallic honeycomb sandwich panel structures to high-intensity dynamic loading. Int J Imp Eng 35 (9): 1063-1074.

[4] Apetre NA, Sankar BV, Ambur DR (2006) Low-velocity impact response of sandwich beams with functionally graded core. Int J Solid Struct 43 (9): 2479-2496.

[5] Li Y, Ramesh, KT, Chin ESC (2001) Dynamic characterization of layered and graded structures under impulsive loading. Int J Solid Struct 38 (34-35): 6045-6061.

[6] Wang E, Gardner N, Shukla A (2009) The blast resistance of sandwich composites with stepwise graded cores. Int J Solid Struct 46: 3492-3502.

[7] Yi J, Boyce MC, Lee GF, Balizer E (2005) Large deformation rate-dependent stress-strain behavior of polyurea and polyurethanes. Poly 47 (1): 319-329.

[8] Amirkhizi AV, Isaacs J, McGee J, Nemat-Nasser S (2006) An experimentallybased constitutive model for polyurea, including pressure and temperature effects. Phil Mag 86 (36): 5847-5866.

[9] Hoo Fatt MS, Ouyang X, Dinan RJ (2004) Blast response of walls retrofitted with elastomer coatings. Struct Mater 15: 129-138.

[10] Roland CM, Twigg JN, Vu Y, Mott PH (2006) High strain rate mechanical behavior of polyurea. Poly 48 (2): 574-578.

[11] Tekalur SA, Shukla A, Shivakumar K (2008) Blast resistance of polyurea based layered composite materials. Comp Struct 84: 271-281.

[12] Bahei-El-Din YA, Dvorak GJ, Fredricksen OJ (2006) A blast-tolerant sandwich plate design with a polyurea interlayer. Int J Solid Struct 43: 7644-7658.

[13] Amini MR, Isaacs JB, Nemat-Nasser S (2010) Experimental investigation of response of monolithic and bilayer plates to impulsive loading. Int J Imp Eng 37: 8289. 
[14] Amini MR, Amirkhizi AV, Nemat-Nasser S (2010) Numerical modeling of response of monolithic and bilayer plates to impulsive loading. Int J Imp Eng 37: 90102.

[15] Amini MR, Isaacs JB, Nemat-Nasser S (2010) Numerical modeling of effect of polyurea on response of steel plates to impulsive loads in direct pressure-pulse experiments. Mech Mater 42: 615-627.

[16] Amini MR, Isaacs JB, Nemat-Nasser S (2010) Investigation of effect of polyurea on response of steel plates to impulsive loads in direct pressure-pulse experiments. Mech Mater 42: 628-639.

http://www.gurit.com/page.asp?section $=00010001002200160009 \&$ sectionTitle=Corec ell\%99+A-Foam+-+For+Hulls+\%26+Dynamic+Loading. Accessed 21 Dec 2010.

http://www.specialty-products.com/polyurea-products/Polyurea-Plural-Component-

Coatings/dragonshield-ht---ercl. Accessed 21 Dec 2010.

[19] Chen W, Zhang B, Forrestal MJ (1998) A split Hopkinson bar technique for low impedance materials. Exp Mech 39 (2): 81-85.

[20] Liang Y, Spuskanyuk AV, Flores SE, Hayhurst DR, Hutchinson JW, McMeeking RM, Evans AG (2007) The response of metallic sandwich panels to water blast. J Appl Mech 74: 81-99.

[21] Tilbrook MT, Deshpande VS, Fleck NA (2006) The impulsive response of sandwich beams: Analytical and numerical investigation of regimes of behavior. J Mech Phys Sol 54: 2242-2280.

[22] Wang E, Shukla A (2010) Analytical and experimental evaluation of energies during shock wave loading. Int J Imp Eng 37: 1188-1196. 


\section{CHAPTER 5}

\section{THE BLAST RESPONSE OF SANDWICH COMPOSITES WITH GRADED CORE: EQUIVALENT CORE LAYER THICKNESS VS EQUIVALENT CORE LAYER MASS}

by

Nate Gardner and Arun Shukla

Under preparation for submission to the Journal of Composites: Part B

Corresponding Author: Arun Shukla

Dynamic Photo Mechanics Laboratory

Department of Mechanical, Industrial and Systems

Engineering

University of Rhode Island

206 Wales Hall, 92 Upper College Rd

Kingston, RI, 02881, USA

Phone: +1-401-874-2283

Email Address: shuklaa@egr.uri.edu 


\begin{abstract}
The dynamic behavior of sandwich composites made of E-glass Vinyl-Ester (EVE) facesheets and Corecell ${ }^{\mathrm{TM}}$ A-series foam were studied using a shock tube apparatus. The materials, as well as the core layer arrangements, and overall specimen dimensions were identical, with the only difference appearing in the core layers; one configuration utilized equivalent core layer thickness, while the other configuration utilized equivalent core layer mass. The foam core itself was layered based on monotonically increasing the acoustic wave impedance of the core layers, with the lowest wave impedance facing the shock loading. During the shock tube testing, highspeed photography system coupled with the optical technique of 3-D Digital Image Correlation (DIC) was utilized to capture the real-time deformation process as well as mechanisms of failure. Post-mortem analysis was carried out to evaluate the overall blast performance of these two configurations. The results indicated that with a decrease in areal density of $\sim 1 \mathrm{~kg} / \mathrm{m}^{2}(5 \%)$ from the sandwich composites with equivalent core layer thickness to the sandwich composites with equivalent core layer mass, an increase in deflection (20\%), in-plain strain (8\%) and velocity (8\%) was observed.
\end{abstract}

Keywords: Sandwich Structures, Functionally Graded Materials, Areal Density, Flexural Stiffness, Blast Loading, High-Speed Photography, 3-D Digital Image Correlation (DIC)

\title{
1. Introduction
}

Sandwich structures have very important applications in the naval and aerospace industry. Due to their construction they have many advantages that include high 
strength/weight ratio, high stiffness/weight ratio, and energy absorption capabilities. Sandwich structures consist of two thin, stiff facesheets, usually the same thickness, separated by a lightweight, thicker core. The facesheets carry almost all of the bending and in-plane loads, while the core helps to stabilize the facesheets and defines the flexural stiffness and out-of-plane shear and compressive behavior. When sandwich structures are subjected to high-intensity impulse loadings, such as air blasts, the core materials play a crucial role in the dynamic behavior and overall structural response. Their properties assist in dispersing the mechanical impulse that is transmitted into the structure, and thus protect anything located behind it [1-3].

Common cores are made of metallic and non-metallic honeycombs, cellular foams, balsa wood, PVC, truss and lattice structures. Extensive research exists in the literature regarding the dynamic response of sandwich structures consisting of the various core materials and geometric structures [3-11]. Dharmasena et al. [3], Zhu et al. [4], and Nurick et al. [5] have tested sandwich structures with a metallic honeycomb core material. Their results indicated that the parameters of core materials can effectively reduce the transmitted impulse and damage of the back facesheet. Radford et al. [6] and Wang et al. [7] have conducted metal foam projectile impact experiments to simulate a blast loading on sandwich structures with metal foam cores. Tagarielli et al. [8] and Atas et al. [9] have investigated the dynamic response of sandwich beams with PVC and balsa wood cores. McShane et al. $[10,11]$ have investigated the underwater blast response of sandwich composites with a prismatic lattice (Y-frame, corrugated), as well as simulated an air blast, using metal foam projectiles, on sandwich composites with a pyramidal lattice cores. These studies have 
indicated that advanced sandwich structures can potentially have significant advantages over monolithic plates of equivalent mass in absorbing the blast energy, whether in air or underwater.

In recent years, functionally graded materials, where the material properties vary gradually or layer by layer within the material itself, have gained much attention. Since the properties of the layered/graded material can be designed and controlled, they show great potential to be an effective core material for energy absorption and blast mitigation [12-18]. Li et al. [15] numerically examined the response of layered and graded metal-ceramic structures under impulsive loadings. It was concluded that the choice of gradation has a great significance on the impact applications and the particular design can exhibit better energy dissipation properties. Apetre et al. [16] numerically investigated the impact response of sandwich beams with a functionally graded core. Their results showed that a reasonable core design can effectively reduce the shear forces and strains within the structure. Consequently, they can mitigate or completely prevent impact damage on sandwich composites. In the previous work done by the authors $[17,18]$, they experimentally investigated the blast resistance of sandwich composites with a functionally graded foam cores. Results indicated that monotonically increasing the wave impedance of the foam core, thus reducing the wave impedance mismatch between successive foam layers, will introduce a stepwise core compression, greatly enhancing the overall blast resistance of sandwich composites. It was also concluded that increasing the number of foam core layers, thus introducing more material interfaces, allows for blast wave (stress wave) attenuation: 
resulting in a time-delay of the peak stress arrival and consequently delaying the time of damage initiation.

In [17] two types of core configurations were studied and the sandwich composites were layered / graded based on the wave impedance of the given foams, i.e. monotonically and non-monotonically. In [18] four types of core configurations were investigated and the sandwich composites had a monotonically graded core based on increasing wave impedance, where the core gradations consisted of one, two, three and four layers respectively. The specimen dimensions and overall thickness were held constant, and each individual core layer was of equivalent thickness, i.e. for two layers of core gradation, each core layer was $19.0 \mathrm{~mm}$, while with four layers of core gradation, each core layer was $9.5 \mathrm{~mm}$.

The current investigation is an extension of the author's previous work and focuses on the blast response of sandwich composites with equivalent core layer mass. By using sandwich composites with equivalent core layer mass, the overall areal density of the specimen is reduced in comparison to its sandwich composite counterpart with equivalent core layer thickness. The quasi-static and dynamic constitutive behaviors of the foam core materials were first studied using a modified SHPB device with a hollow transmitted bar. The sandwich composites were then fabricated and subjected to shock wave loading generated by a shock tube. The materials, as well as the core layer arrangements, and overall specimen dimensions were identical; the only difference arises in the core layers, where one configuration has equivalent core layer thickness, and the other configuration has equivalent core layer mass. The shock pressure profiles and real-time deflection images were 
carefully analyzed to reveal the mechanisms of dynamic failure of these sandwich composites. Digital Image Correlation (DIC) analysis was implemented to investigate the real-time full-field deflection, in-plane-strain, and particle velocity on the back face of the specimens. Post-mortem analysis was also carried out to better evaluate the overall blast performance of the specimens (structural integrity).

\section{Material and Specimen}

\subsection{Skin (Facesheet) and Core Materials}

The facesheet materials that were utilized in this study are E-glass Vinyl-Ester (EVE) composites. The woven roving E-glass fibers of the skin material consisted of 8 layers of plain weave fabric placed in a quasi-isotropic layout $[0 / 45 / 90 /-45]_{s}$. The fibers were made of the $0.61 \mathrm{~kg} / \mathrm{m}^{2}$ areal density plain weave. The resin system used was Ashland Derakane Momentum 8084, with the front and back skins (facesheets) consisting of identical layups and materials. The EVE composite consisted of a 55\% / $45 \%$ volume fraction of glass (fiber) to resin, as determined using proper ASTM standard D 2584. Fig. 1 shows a schematic of the sandwich composite with skin and core materials.

The core materials used in the present study are Corecell ${ }^{\mathrm{TM}} \mathrm{A}$-series styrene acrylonitrile (SAN) foams, which are manufactured by Gurit SP Technologies specifically for marine sandwich composite applications. The two types of Corecell ${ }^{\mathrm{TM}}$ A-series foam that were used in present study were A300 and A800. Table 1 lists important material properties of the two foams from the manufacturer's data [19], as well as the material properties of the facesheet [18]. The material properties of the 
facesheet and the core materials were determined using proper ASTM standards, D 3410 and D 1621 respectively.

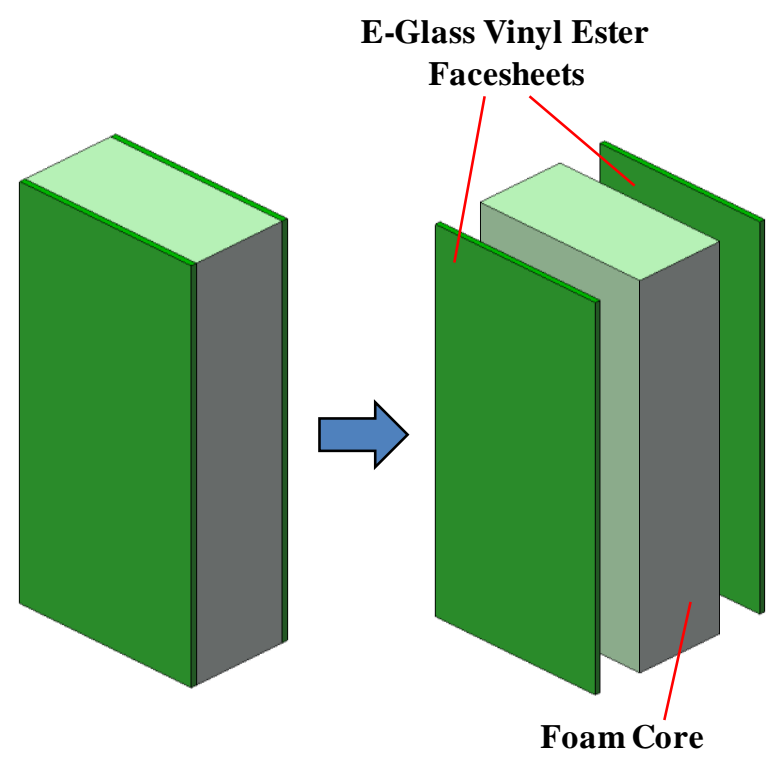

Fig. 1 Schematic of sandwich composite with skin and core

Table 1. Quasi-static material properties of foam core [18] and EVE facesheet

\begin{tabular}{ccccc}
\hline & $\begin{array}{c}\text { Nominal } \\
\text { Density, } \boldsymbol{\rho} \\
\left(\mathrm{kg} / \mathrm{m}^{3}\right)\end{array}$ & $\begin{array}{c}\text { Compressive } \\
\text { Modulus, E } \\
(\mathrm{MPa})\end{array}$ & $\begin{array}{c}\text { Compressive } \\
\text { Strength, } \boldsymbol{\sigma}_{\mathbf{y}} \\
(\mathrm{MPa})\end{array}$ & $\begin{array}{c}\text { Acoustic Wave } \\
\text { Impedance } \\
\left(\mathrm{kg} / \mathrm{m}^{2} \mathrm{~s}\right)\end{array}$ \\
\hline $\mathbf{A 3 0 0}$ & 58.5 & 32 & 0.5 & $4.3 \times 10^{4}$ \\
$\mathbf{A 8 0 0}$ & 150 & 117 & 2.1 & $13.2 \times 10^{4}$ \\
$\begin{array}{c}\text { E-glass } \\
\text { Vinyl-Ester } \\
\text { Composite }\end{array}$ & 1800 & $\begin{array}{c}13600 \\
{[\text { longitudinal] }} \\
3900\end{array}$ & 220 & $\begin{array}{c}4.9 \times 10^{6} \\
{[\text { longitudinal] }} \\
2.6 \times 10^{6} \\
{[\text { transverse] }}\end{array}$ \\
\hline
\end{tabular}

In Table 1, the A300 foam has a lower nominal density ( $\rho)$ and compressive modulus (E) than the A800 foam. Since both the nominal density and the compressive modulus are lower in the A300 foam than the A800 foam, the one-dimensional acoustic wave impedance $(\mathrm{Z})$ is also lower, and shows the following relationship, 


$$
\begin{gathered}
Z=\rho C=\rho \sqrt{E / \rho} \\
Z_{\mathrm{A} 300}<Z_{800}
\end{gathered}
$$

The cell structures for the two foams are similar and the only difference appears in the cell wall thickness and node sizes, which accounts for the different densities of the foams. The SEM images of the cell microstructures can be seen in Fig. 2.

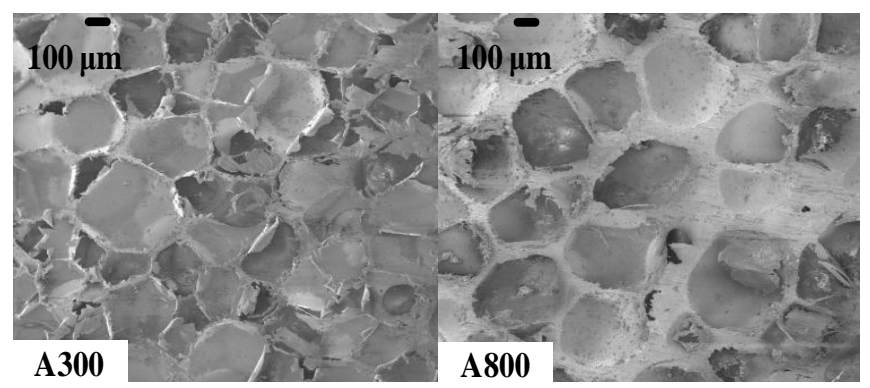

Fig. 2 Cell microstructure of foam core layers [18]

\subsection{Sandwich Panels with Equivalent Core Layer Thickness and Equivalent Core}

\section{Layer Mass}

The Vacuum Assisted Resin Transfer Molding (VARTM) process was utilized to fabricate the sandwich specimens. During the VARTM process, the sandwich specimens were infused under the same conditions, i.e. temperature, humidity, and vacuum pressure $(760 \mathrm{mmHg}(1 \mathrm{~atm}))$, with the same volume of resin. The overall dimensions for the samples were approximately $102 \mathrm{~mm}$ wide, $254 \mathrm{~mm}$ long and 48 mm thick. The foam core itself was approximately $38 \mathrm{~mm}$ thick, while the skin thickness was approximately $5 \mathrm{~mm}$.

For the sandwich composites utilized in this study, two different core layer arrangements were investigated (as shown in Fig. 3). Both configurations consisted of two core layers of foam, A300 and A800 respectively, and were arranged based on monotonically increasing the acoustic wave impedance (A300/A800). It should be 
noted that the first core layer of foam, A300, is the one first exposed to the shock wave loading. For the sandwich composites with equivalent core layer thickness, both foam layers had equivalent layer thickness $(19 \mathrm{~mm})$, resulting in an overall areal density of approximately $18.5 \mathrm{~kg} / \mathrm{m}^{2}$. For the sandwich composites with equivalent core layer mass, the mass of the individual foam layers was equivalent. The following equations were used to obtain the required foam core thickness in order to equalize the mass of the A300 and A800 foam core layers,

First the overall thickness, $\mathrm{t}_{\text {foam_overal }}$, must remain equal,

$$
\mathrm{t}_{\mathrm{A} 300}+\mathrm{t}_{\mathrm{A} 800}=\mathrm{t}_{\text {foam_overall }}=38 \mathrm{~mm}=38 \times 10^{-3} \mathrm{~m}
$$

To maintain equivalent mass, $\mathrm{m}$,

$$
\mathrm{m}=(\mathrm{V})(\rho)=\left(\mathrm{V}_{\mathrm{A} 300}\right)\left(\rho_{\mathrm{A} 300}\right)=\left(\mathrm{V}_{\mathrm{A} 800}\right)\left(\rho_{\mathrm{A} 800}\right)
$$

where $\rho$ is the nominal density and $\mathrm{V}$ is the volume

$$
\mathrm{V}=(\mathrm{A})(\mathrm{t})
$$

where $\mathrm{A}$ is the individual foam layer area, then Eq. (4) becomes

$$
\left(\mathrm{A}_{\mathrm{A} 300}\right)\left(\mathrm{t}_{\mathrm{A} 300}\right)\left(\rho_{\mathrm{A} 300}\right)=\left(\mathrm{A}_{\mathrm{A} 800}\right)\left(\mathrm{t}_{\mathrm{A} 800}\right)\left(\rho_{\mathrm{A} 800}\right)
$$

Note, since $\mathrm{A}_{\mathrm{A} 300}=\mathrm{A}_{\mathrm{A} 800}$

$$
\left(\mathrm{t}_{\mathrm{A} 300}\right)\left(\rho_{\mathrm{A} 300}\right)=\left(\mathrm{t}_{\mathrm{A} 800}\right)\left(\rho_{\mathrm{A} 800}\right)
$$

Substituting Eq. (3) into Eq. (7) yields

$$
\left(\mathrm{t}_{\mathrm{A} 300}\right)\left(\rho_{\mathrm{A} 300}\right)=\left(38 \mathrm{e}^{-3} \mathrm{~m}-\mathrm{t}_{\mathrm{A} 300}\right)\left(\rho_{\mathrm{A} 800}\right)
$$

From Table $1, \rho_{\mathrm{A} 300}=58.5 \mathrm{~kg} / \mathrm{m}^{3}$ and $\rho_{\mathrm{A} 800}=150 \mathrm{~kg} / \mathrm{m}^{3}$, which yields

$$
\left(58.5 \mathrm{~kg} / \mathrm{m}^{3}\right)\left(\mathrm{t}_{\mathrm{A} 300}\right)=\left(150 \mathrm{~kg} / \mathrm{m}^{3}\right)\left(38 \mathrm{e}^{-3} \mathrm{~m}-\mathrm{t}_{\mathrm{A} 300}\right)
$$

and expanding becomes

$$
\left(58.5 \mathrm{~kg} / \mathrm{m}^{3}\right)\left(\mathrm{t}_{\mathrm{A} 300}\right)=\left(5.7 \mathrm{~kg} / \mathrm{m}^{2}\right)-\left(150 \mathrm{~kg} / \mathrm{m}^{3}\right)\left(\mathrm{t}_{\mathrm{A} 300}\right)
$$


Rearranging, and solving for $\mathrm{t}_{\mathrm{A} 300}$

$$
\begin{gathered}
\left(208.5 \mathrm{~kg} / \mathrm{m}^{3}\right)\left(\mathrm{t}_{\mathrm{A} 300}\right)=\left(5.7 \mathrm{~kg} / \mathrm{m}^{2}\right) \\
\mathrm{t}_{\mathrm{A} 300}=0.027 \mathrm{~m}=27 \mathrm{~mm}
\end{gathered}
$$

Substituting Eq. (1) yields,

$$
27 \mathrm{~mm}+\mathrm{t}_{\mathrm{A} 800}=38 \mathrm{~mm}
$$

and thus,

$$
\mathrm{t}_{\mathrm{A} 800}=11 \mathrm{~mm}
$$

Therefore, in order for the individual core layers to have equivalent mass, the thickness of the A300 and A800 foam would need to be $27.0 \mathrm{~mm}$ and $11.0 \mathrm{~mm}$ respectively. Due to specific dimensions and material availability from the manufacturer [19], the thickness of the A300 and A800 foam were $25.4 \mathrm{~mm}$ and 12.7 $\mathrm{mm}$ respectively. This resulted in panels with an overall areal density of approximately $17.6 \mathrm{~kg} / \mathrm{m}^{2}$.

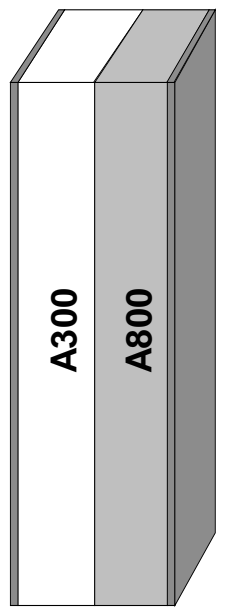

Equivalent Thickness Equivalent Mass

(a) Different core layer arrangements

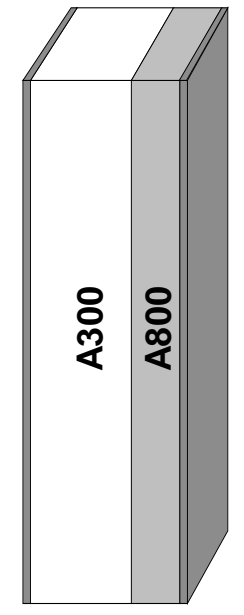

Fig. 3 Specimen design and core configuration 


\section{Experimental Set-up and Procedure}

\subsection{Modified Split Hopkinson Pressure Bar with Hollow Transmission Bar}

A Split Hopkinson Pressure Bar (SHPB) is the most common device used to measure the dynamic constitutive properties of materials. Due to the low-impedance of Corecell ${ }^{\mathrm{TM}}$ foam materials, dynamic experiments for the core materials were performed with a modified SHPB device with a hollow transmission bar to increase the transmitted signal intensity. A sketch of the modified SHPB device and typical pulse profiles are given in Fig. 4. It has a 305 mm-long striker, 1600 mm-long incident bar and $1447 \mathrm{~mm}$-long transmission bar. All of the bars are made of a 6061 aluminum alloy. The nominal outer diameters of the solid incident bar and hollow transmission bar are both $19.05 \mathrm{~mm}$. The hollow transmission bar has a $16.51 \mathrm{~mm}$ inner diameter. At the head and at the end of the hollow transmission bar, end caps made of the same material as the bar were press fitted into the hollow tube. By applying pulse shapers, the effect of the end caps on the stress waves can be minimized. The details of the analysis and derivation of equations for analysis of experimental data can be found in Chen et. al [20].

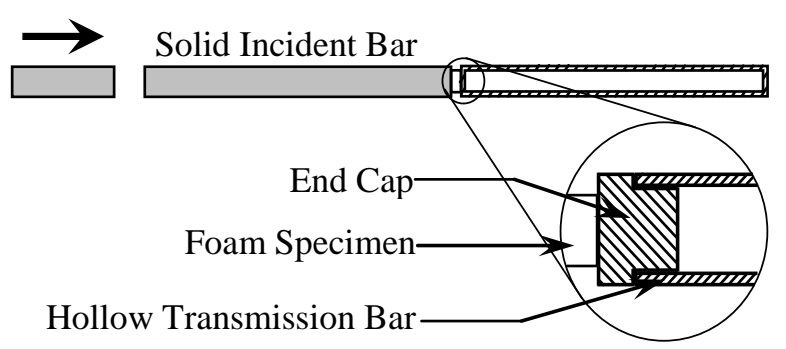

(a) Modified SHPB device

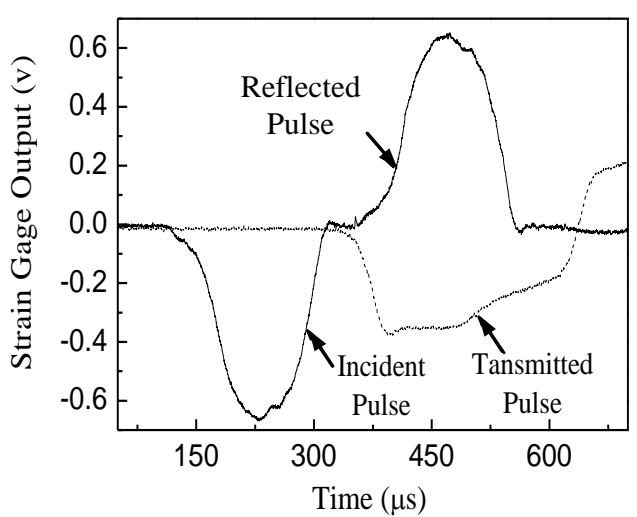

(b) Typical pulse profiles

Fig. 4 Sketch of modified SHPB device with hollow transmission bar and typical pulse profiles 


\subsection{Shock Tube}

The shock tube apparatus used to obtain the controlled dynamic loading is shown in Fig. 5(a). Shock tubes offer the advantages of plane wave fronts, wave parameters that are easily controllable and repeatable, and uniform loading over shock tube muzzle diameter [21]. A complete description of the shock tube and its calibration can be found in ref. [22]. In principle, the shock tube consists of a long rigid cylinder, divided into a high-pressure driver section and a low pressure driven section, which are separated by a diaphragm. By pressurizing the high-pressure driver section, a pressure difference across the diaphragm is created. When this pressure differential reaches a critical value, the diaphragm ruptures. The subsequent rapid release of gas creates a shock wave, which travels down the shock tube to impart shock loading on the specimen at the muzzle end.

When the shock wave impacts the specimen located at the end of the muzzle, the wave is reflected at a higher pressure than that of the incident shock pressure. The theoretical details on the equations for shock tubes have been previously established in the literature [23]. There are four basic theoretical assumptions which are used to describe the gas flow in shock tube:

1. The gas flow is one-dimensional.

2. The gas is ideal and has constant specific heats.

3. Heat transfer and viscosity effects are neglected.

4. Diaphragm rupture is instantaneous and does not disturb the subsequent gas flow. 
Using conservation of energy, mass, and momentum as described by Wright [23], the following relationships for pressure, temperature and density across a shock front can be derived:

$$
\begin{gathered}
\frac{P_{2}}{P_{1}}=\frac{2 \gamma M_{1}^{2}-(\gamma-1)}{\gamma+1} \\
\frac{T_{2}}{T_{1}}=\frac{\left\{2 \gamma M_{1}^{2}-(\gamma-1)\right\}\left\{(\gamma-1) M_{1}^{2}+2\right\}}{(\gamma+1)^{2} M_{1}^{2}} \\
\frac{\rho_{2}}{\rho_{1}}=\frac{M_{1}^{2}(\gamma+1)}{(\gamma-1) M_{1}^{2}+2}
\end{gathered}
$$

where, $\mathrm{P}_{1}, \mathrm{~T}_{1}$ and $\rho_{1}$, are pressure, temperature and density ahead of the shock front and, $\mathrm{P}_{2}, \mathrm{~T}_{2}$ and $\rho_{2}$, are the pressure, temperature and density behind the shock front, $\gamma$ is the adiabatic gas constant, and $\mathrm{M}_{1}$ is the mach number of the shock wave relative to the driven gas. The pressure imparted on the specimen can be controlled by varying the above parameters in equations 9, 10, and 11. Different gases, such as nitrogen, and helium, were used in the shock tube and it was found that helium is the most suitable gas to replicate blast loading conditions and also offered the added advantage of repeatability.

The shock tube apparatus utilized in the present study has an overall length of 8 $\mathrm{m}$, consisting of a driver, driven, converging and muzzle section. The diameter of the driver and driven section is $0.15 \mathrm{~m}$. The final muzzle diameter is $0.07 \mathrm{~m}$. Fig. $5 \mathrm{~b}$ shows detailed dimensions and locations of the muzzle, specimen, supports and the pressure sensors (PCB102A). The sensors are mounted at the end of the muzzle section to measure the incident pressure and the reflected pressure during the experiment. The final muzzle diameter is $0.076 \mathrm{~m}$. The distance between the two sensors is $0.160 \mathrm{~m}$ and the distance between the second sensor and the end of the 
muzzle is $\sim 0.020 \mathrm{~m}$. The specimen was placed in the supports and positioned close to the end of the muzzle. These support fixtures ensure simply supported boundary conditions with a $0.152 \mathrm{~m}$ span.

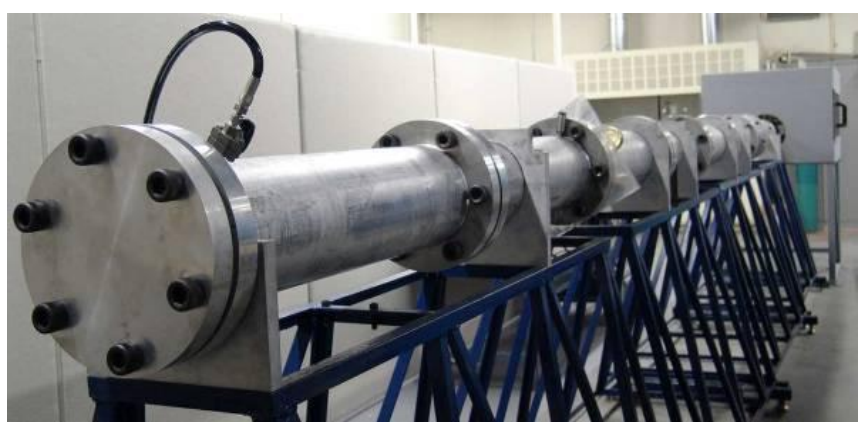

(a) Shock tube facility at URI

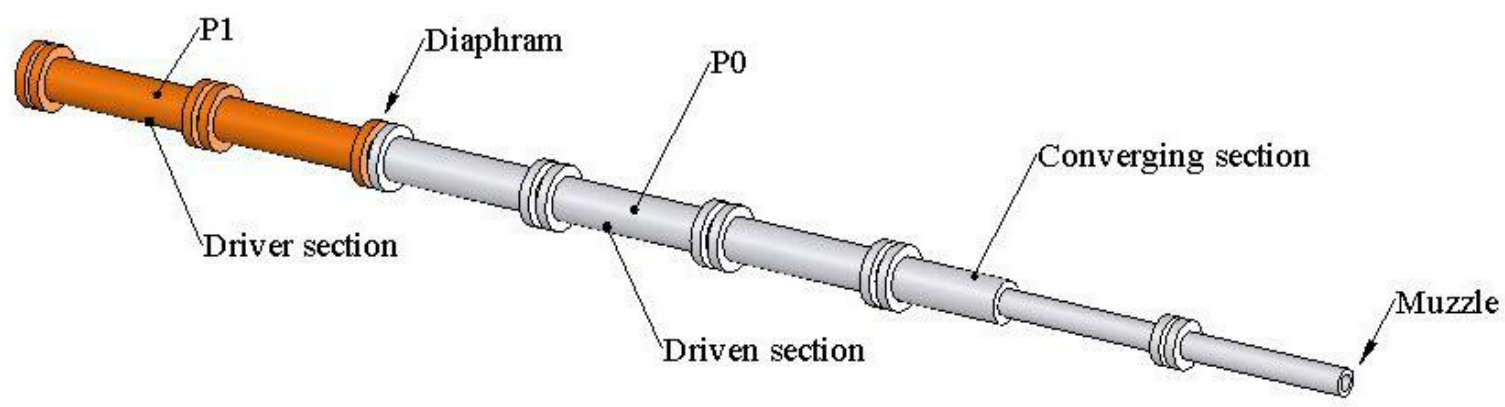

(b) Schematic of shock tube

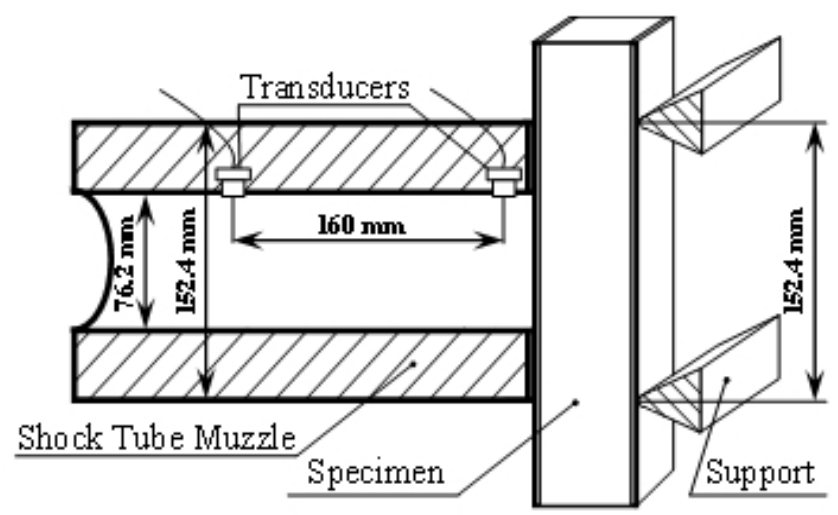

(c) Detailed dimensions of the muzzle

Fig. 5 Shock tube apparatus 


\subsection{High-speed Photography Systems}

Two high-speed photography systems were used in the present study, as shown in Fig. 6. A high-speed 3-D Digital Image Correlation (DIC) system, consisting of two high-speed digital cameras [Photron SA1], was placed facing the back side of the specimen to obtain the real-time full-field in-plane strain, along with out-of-plane deflection and velocity of the back facesheet. A randomized speckle pattern was placed directly on the back facesheet of the sandwich composite to ensure good contrast of the images. Another high-speed digital camera, [Photron SA1], was placed perpendicular to the side surface of the specimen to capture the side-view deformation images and mechanisms of failure. A framing rate of 20,000 fps was utilized which gives an interframe time of approximately $50 \mu \mathrm{s}$.

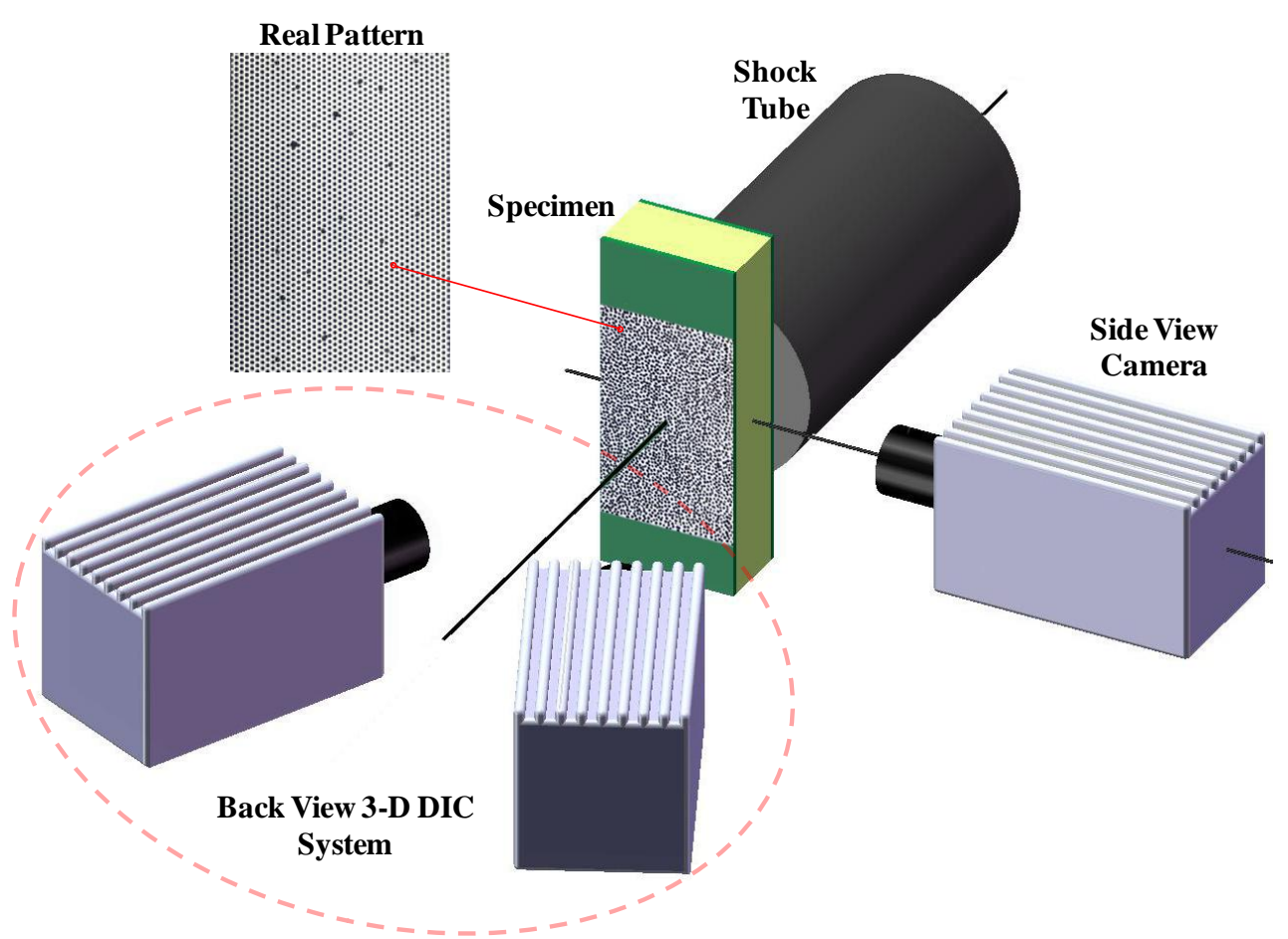

Fig. 6 High-speed photography set-up (Back-view DIC and side-view)

The Digital Image Correlation (DIC) technique is utilized to capture the real-time full-field response of the back face of the sandwich composite panels. It is a non- 
intrusive, optical technique which allows for the capturing of the real-time dynamic response of sandwich composites through the use of high-speed photography and specialized software (PFV). In order to capture the three-dimensional response of the specimens, two cameras must be used in stereo configuration. This means the cameras must be calibrated and have synchronized image recording throughout the entire blast event. The calibration of the cameras is performed by placing a grid containing a known pattern of points (dots) in the exact location as to where the specimen will be placed during the experiment. This grid is then translated and rotated both in and outof-plane, while manually recording a series of images. Due to the fact that the grid pattern has predetermined spacing, the coordinates of the center of each point (dot) is extracted from each image. Since the coordinate location of each dot is extracted uniquely for each camera, this allows for a correspondence of the coordinate system between cameras (Tiwari et al. [24]). DIC post-processing is performed utilizing the image pairs that are recorded during the blast loading event. UsingVIC-3D software package, as distributed by Correlated Solutions, common pixel subsets of the randomized speckle pattern are matched between the deformed images and the undeformed image. By matching the pixel subsets of the random speckle pattern, the three-dimensional location of distinct points on the face of the panel throughout time is obtained. This technique has been applied as a full-field measurement technique in many applications, including shock loading (Tiwari, et al. [25])

\subsection{Experimental Procedure and Parameters}

In the present study, a simply stacked diaphragm of 5 plies of $0.254 \mathrm{~mm}$ mylar sheets, with a total thickness of $1.270 \mathrm{~mm}$, was utilized to generate an impulse loading 
on the specimen with an incident peak pressure of approximately $1 \mathrm{MPa}$, a reflected peak pressure of approximately $5 \mathrm{MPa}$ and a shock wave speed of approximately 1000 $\mathrm{m} / \mathrm{s}$. For each configuration, at least three samples were tested. A typical pressure profile obtained from the transducer closest to the specimen $(\sim 0.020 \mathrm{~m}$ away $)$ can be seen in Fig. 7. It should be noted that both pressure transducers were utilized to obtain the shock wave history, i.e. incident / reflected pressure and incident / reflected velocity. However, only the pressure transducer closest to the specimen was utilized to obtain the pressure applied on the specimen.

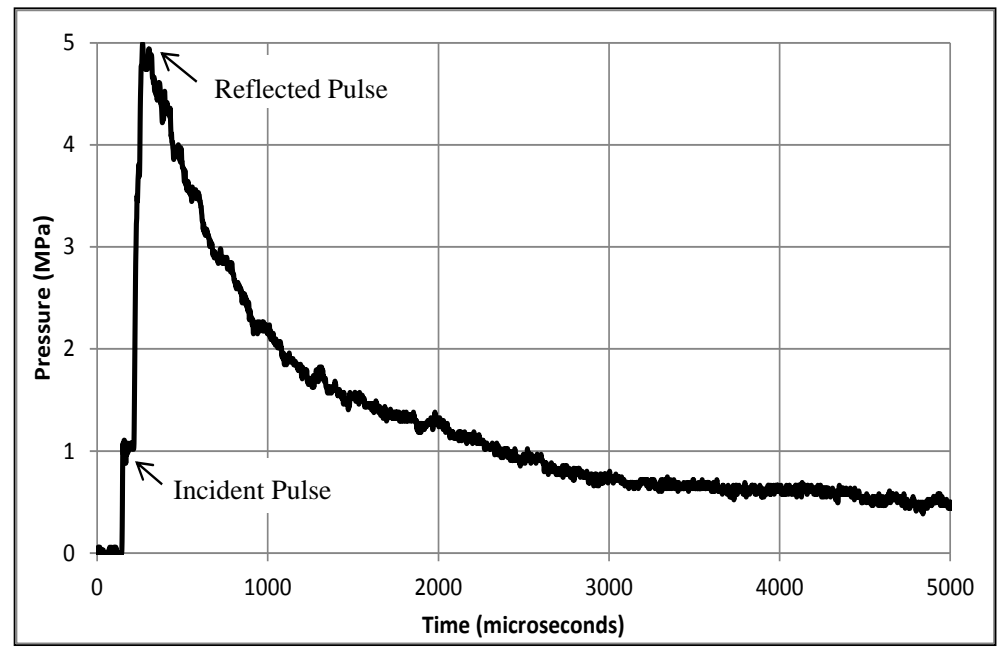

Fig. 7 Incident and reflected pressure profile

\section{Experimental Results and Discussion}

\subsection{Dynamic Behavior of Core Material}

Fig.8 shows the quasi-static (Fig. 8a) and high strain rate (Fig. 8b) compressive behavior of the different types of Corecell ${ }^{\mathrm{TM}}$ A-series foams [18]. For the quasi-static compressive behavior (Fig. 8a), the stress-strain curves show three deformation regions; (I) the linear elastic region, (II) the plateau stress (plastic yielding) region and (III) the densification region [26]. For high strain rate compressive behavior, the 
stress-strain curves also show the three deformation regions, even though the densification region is much harder to achieve. Note the plateau stress regions for both instances have a large strain range.

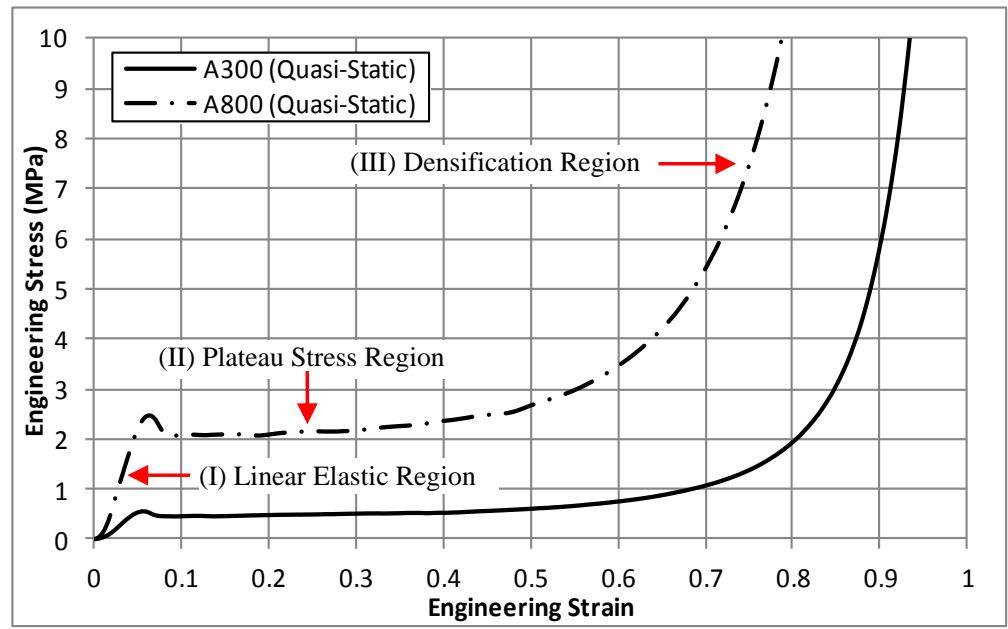

(a) Quasi-Static

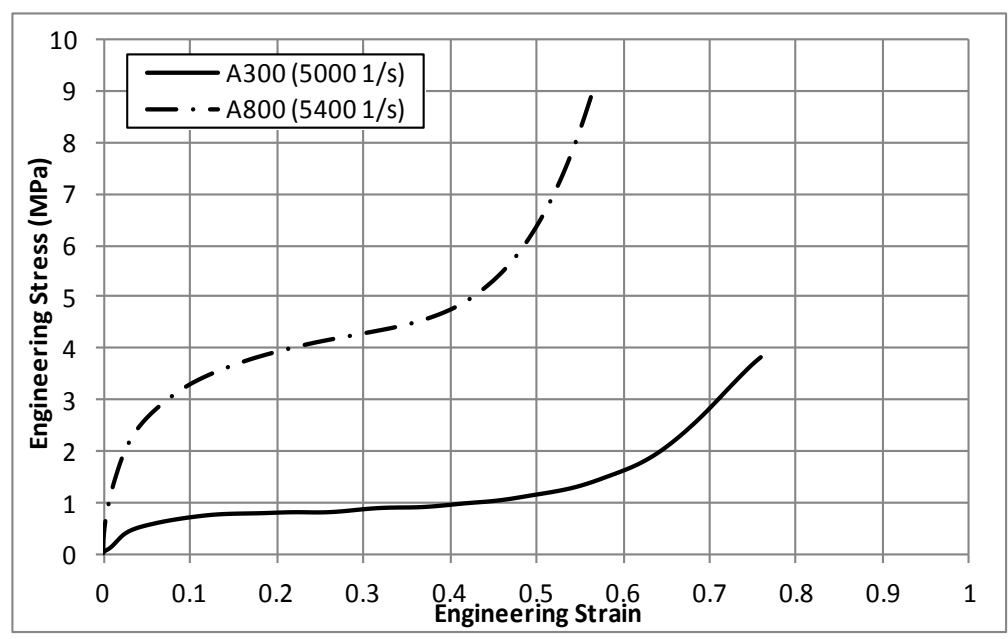

(b) Dynamic

Fig. 8 Compressive behavior of both types of Corecell ${ }^{\mathrm{TM}}$ A-series foams [18]

As seen in Fig.8, the quasi-static and dynamic stress-strain responses have an obvious trend for the different types of foams. Lower density foam has a lower strength and stiffness, as well as a larger strain range for the plateau stress. The high strain rate yield stresses and plateau (flow) stresses are much higher than the quasi- 
static ones for the same type of foams. Table 2 shows the quasi-static and high strain rate plateau stresses (measured in the plateau stress region).

Table 2. Flow stresses (plateau) of both Corecell ${ }^{\mathrm{TM}}$ A-series foams

\begin{tabular}{ccc}
\hline Core Layer & $\mathbf{A 3 0 0}$ & $\mathbf{A 8 0 0}$ \\
\hline $\begin{array}{c}\text { Quasi-Static Yield } \\
\text { Stresses (MPa) }\end{array}$ & 0.48 & 2.08 \\
$\begin{array}{c}\text { High Strain-Rate } \\
\text { Yield Stresses } \\
(\mathrm{MPa})\end{array}$ & 0.8 & 4.00 \\
\hline
\end{tabular}

The dynamic strength of A800 foam increases approximately $100 \%$ in comparison to its quasi-static strength, while the dynamic strength of A300 foam increases approximately $50 \%$ in comparison to its quasi-static strength. The improvement of the mechanical behavior from quasi-static to high strain-rates in these core materials, as well as their long stress plateaus, signifies their ability to absorb large amounts of energy under high strain-rate dynamic loading. Therefore, they show great potential in being used as core materials in sandwich structures subjected to high intensity air blasts.

\subsection{Blast Response of Sandwich Composites with Equivalent Core Layer Thickness and Equivalent Core Layer Mass}

\subsubsection{Real-time Deformation}

The real-time observations of the transient behavior for both sandwich composite panels subjected to shock wave loading are shown in Fig. 9. The shock wave (pressure wave) propagates from the right side of the image to the left side and some detailed deformation mechanisms are pointed out in the figures. It should be noted that the time 
scheme used to represent the images in each configuration is identical. Therefore, for each of the four configurations investigated, the images are correlated based on the same time per frame. This allows for a better comparison between the different configurations in regards to the failure mechanisms and extent of damage observed.

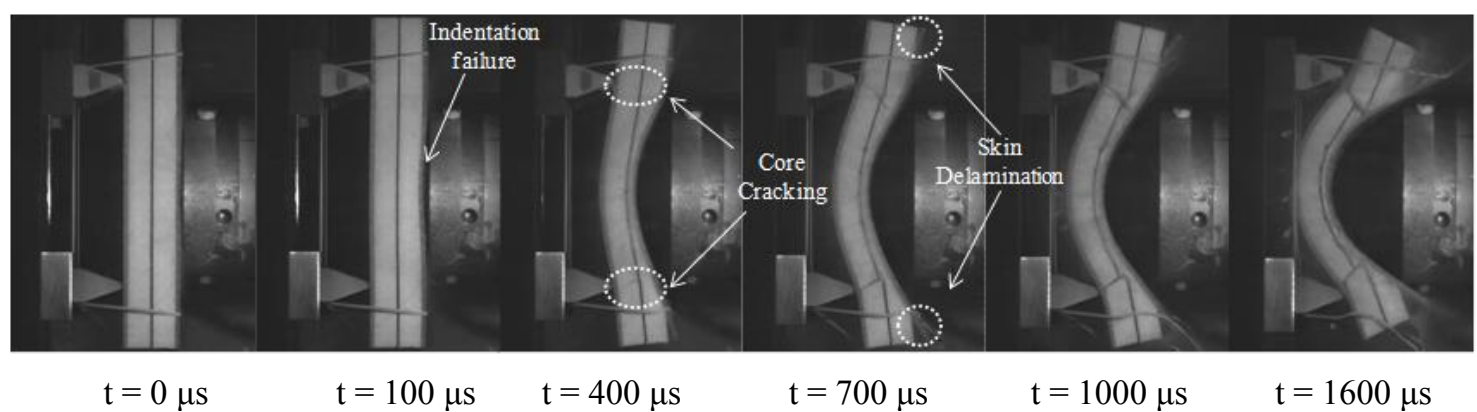

(a) Equivalent Thickness

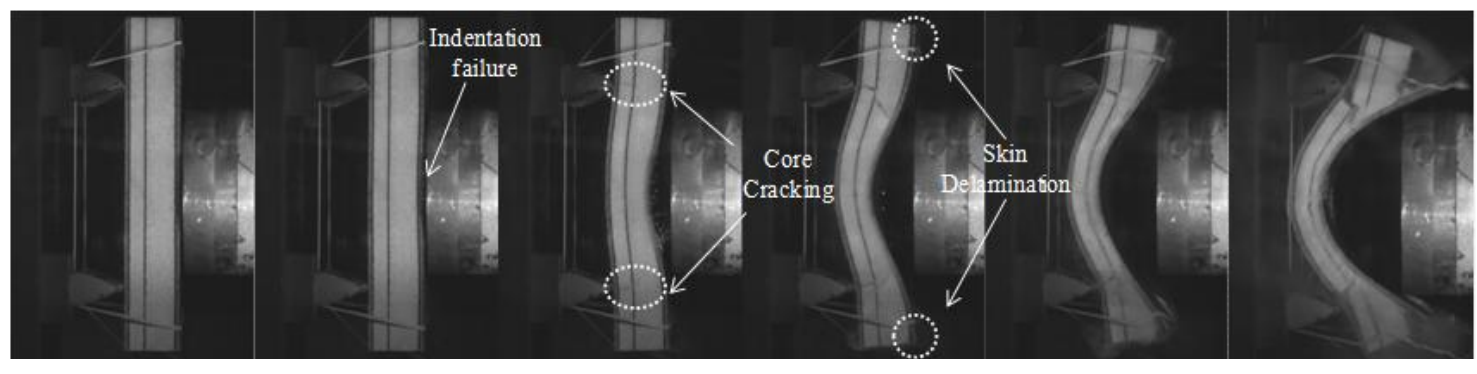

$\mathrm{t}=0 \mu \mathrm{s} \quad \mathrm{t}=100 \mu \mathrm{s} \quad \mathrm{t}=400 \mu \mathrm{s} \quad \mathrm{t}=700 \mu \mathrm{s} \quad \mathrm{t}=1000 \mu \mathrm{s} \quad \mathrm{t}=1600 \mu \mathrm{s}$

(b) Equivalent Mass

Fig. 9 High-speed images for both core configurations

For the sandwich composites with equivalent core layer thickness, as shown in Fig. 9a, it can be observed that at $\mathrm{t}=100 \mu$ s indentation failure of the core has initiated. This means that compression has initiated in the first core layer of foam (A300). By $\mathrm{t}=400 \mu \mathrm{s}$, the A300 layer has continued to compress and core cracking has initiated in the A800 layer where the supports are located. By $\mathrm{t}=700 \mu$ s more core cracking can be observed, as well as skin delamination between the front skin and the first core layer of foam (located at the top and bottom of the specimen). Also at this time, the core compression in the first layer of foam (A300) has reached a 
maximum $(13 \mathrm{~mm})$, approximately $75 \%$ of its original layer thickness $(19 \mathrm{~mm})$. After this time, the response of the specimen is global bending and by $t=1600 \mu$ s, no new failure mechanisms were observed. Also the core cracks have propagated completely through the foam core to the front facesheet, and there is heavy skin delamination between the front facesheet and the first core layer of foam.

For the sandwich composites with equivalent core layer mass, as shown in Fig. $9 \mathrm{~b}$, it can be observed that at $\mathrm{t}=100 \mu \mathrm{s}$ indentation failure of the core has initiated. This means that compression has initiated in the first core layer of foam (A300). By $t$ $=400 \mu \mathrm{s}$, the A300 layer has continued to compress and core cracking has initiated in the A800 layer where the supports are located. By $t=700 \mu$ s more core cracking can be observed, as well as skin delamination between the front skin and the first core layer of foam (located at the top and bottom of the specimen). By $t=1000 \mu \mathrm{s}$, the damage mechanisms have continued to propagate, and the core compression in the first layer of foam (A300) has reached a maximum (19 mm), approximately $75 \%$ of its original layer thickness $(25.4 \mathrm{~mm})$. After this time, the response of the specimen is global bending and by $\mathrm{t}=1600 \mu \mathrm{s}$, no new failure mechanisms were observed. Also the core cracks have propagated completely through the foam core to the front facesheet, and there is heavy skin delamination between the front facesheet and the first core layer of foam.

It was observed in both configurations, equivalent core layer thickness and equivalent core layer mass, that the deformation mechanisms were identical. Both configurations exhibited a double-winged deformation shape which means both configurations were under shear loading. Indentation failure was followed by core 
compression of the first layer of foam (A300) and core cracking, and finally skin delamination between the front facesheet and foam core. The extent of the damage mechanisms varies between configurations, but the time at which the damage mechanisms were observed is identical.

\subsubsection{Deflection}

The mid-point deflections of each configuration and all of its constituents were obtained from the high-speed images, and a typical response can be seen in Fig. 10. For the sandwich composites with equivalent core layer thickness (Fig. 10a), it can be seen that at $\mathrm{t}=1600 \mu \mathrm{s}$ the front face (front skin), interface 1 (between first and second core layer) and the back face (back skin) deflect to approximately $46 \mathrm{~mm}, 33$ $\mathrm{mm}$ and $33 \mathrm{~mm}$ respectively. Since the A300 foam core layer is located between the front skin and interface 1 , the difference in deflection between the front skin and interface 1 indicates the total amount of compression in the A300 layer. Therefore, it is evident that the A300 foam compresses approximately $13 \mathrm{~mm}$, which is $75 \%$ of its original thickness $(19 \mathrm{~mm})$. Also note that the deflection curves for interface 1 and the back face follow the same trend and deflect to the same value at $\mathrm{t}=1600 \mu \mathrm{s}(33 \mathrm{~mm})$. Therefore, no compression was observed in the A800 core layer of foam.

For the sandwich composites with equivalent core layer mass, the mid-point deflections are shown in Fig. 10b. It can be seen that at $\mathrm{t}=1600 \mu \mathrm{s}$, the front skin, interface 1, and the back skin deflect to approximately $60 \mathrm{~mm}, 41 \mathrm{~mm}$ and $41 \mathrm{~mm}$ respectively. Since the A300 foam core layer is located between the front skin and interface 1 , the difference in deflection between the front skin and interface 1 shows the amount of compression in the A300 layer. Therefore, it can be observed that the 
A300 foam compresses approximately $19 \mathrm{~mm}$, which is $75 \%$ of its original thickness $(25.4 \mathrm{~mm})$. Also note that the deflection curves for interface 1 and the back face follow the same trend and deflect to the same value at $\mathrm{t}=1600 \mu \mathrm{s}(41 \mathrm{~mm})$. Therefore, no compression was observed in the A800 core layer of foam.
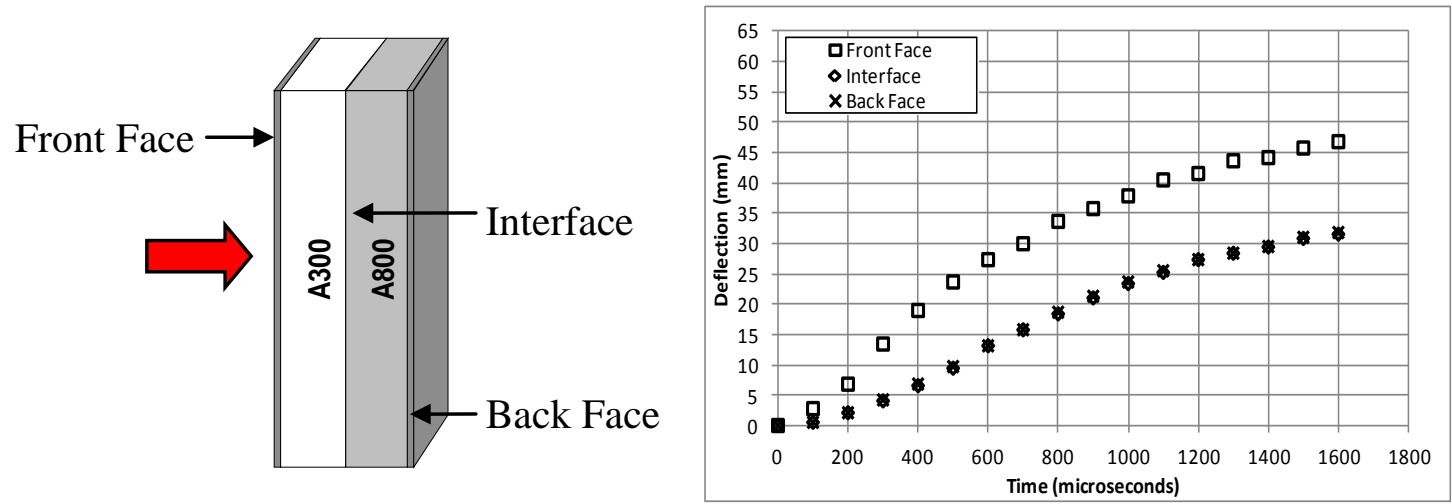

(a) Equivalent Thickness
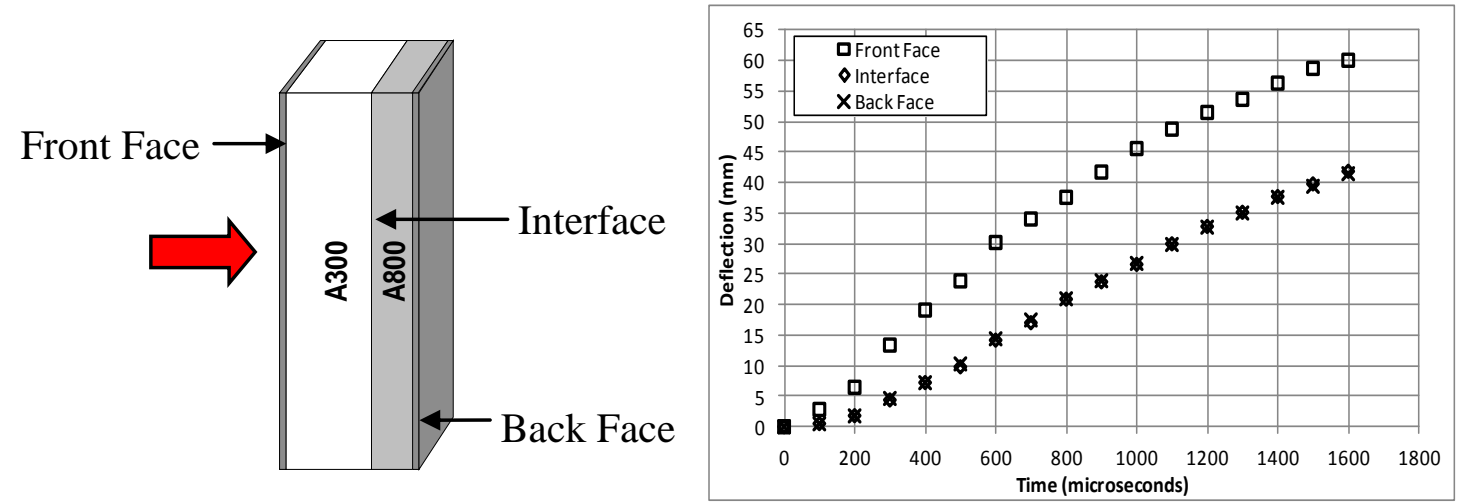

(b) Equivalent Mass

Fig. 10 Mid-point deflection of both configurations

Fig. 11 shows a comparison of the mid-point deflections for the back face of each configuration. It can be seen in the figure that at $\mathrm{t}=1600 \mu \mathrm{s}$, the back face of the sandwich composites with equivalent core layer thickness deflects approximately $20 \%$ less than the back face of the sandwich composites with equivalent core layer mass, i.e. $33 \mathrm{~mm}$ and $41 \mathrm{~mm}$ respectively. 


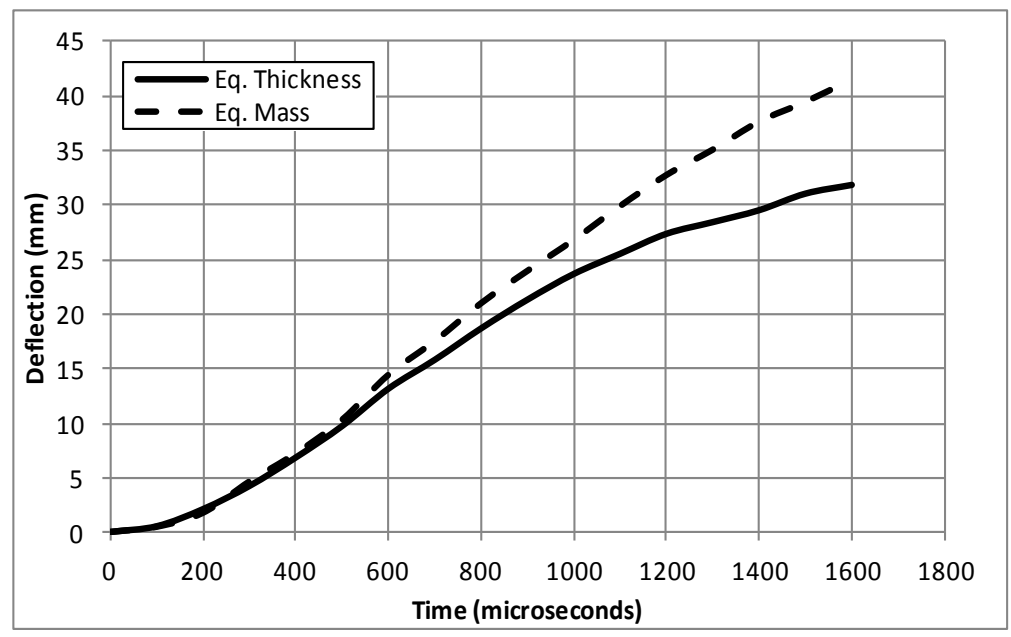

Fig. 11 Comparison of mid-point deflections of the back face of both configurations

From the deflection data of each interface in Fig. 10, the deformation of each core layer along the mid- line (line of symmetry) can be obtained by subtracting the core layers' back side deflection from the core layers' front side deflection. Sequentially, the strain along the line of symmetry of each core layer can be obtained using the following equation,

$$
\text { strain }=\varepsilon=\frac{(\Delta \mathrm{l})}{1_{\text {original }}}
$$

where, $l_{\text {original }}$ is the original thickness of the each core layer.

The strain history of the A300 foam core layer of both configurations, as calculated from Eq. (12) using the mid-point deflection data from Fig. 10, is shown in Fig. 12. Since there is no compression in the A800 foam core layer, its strain history is not shown here. It can be seen that in both configurations, the A300 foam layer exhibits approximately the same amount of maximum strain, $75 \%-80 \%$, however the time in which the maximum strain was reached varied. For the sandwich composites with equivalent core layer thickness, the maximum strain value was achieved by $\sim \mathrm{t}=$ $500 \mu \mathrm{s}$. For the sandwich composites with equivalent core layer mass, the maximum 
strain value was achieved by $\sim \mathrm{t}=1000 \mu \mathrm{s}$. Therefore, since the maximum strain value of the A300 foam layer for the sandwich composites with equivalent core layer mass was achieved in half the time in comparison to that of the sandwich composites with equivalent core layer mass, the behavior of the stiffer, A800 foam layer came into effect much earlier in the panels' deformation history.

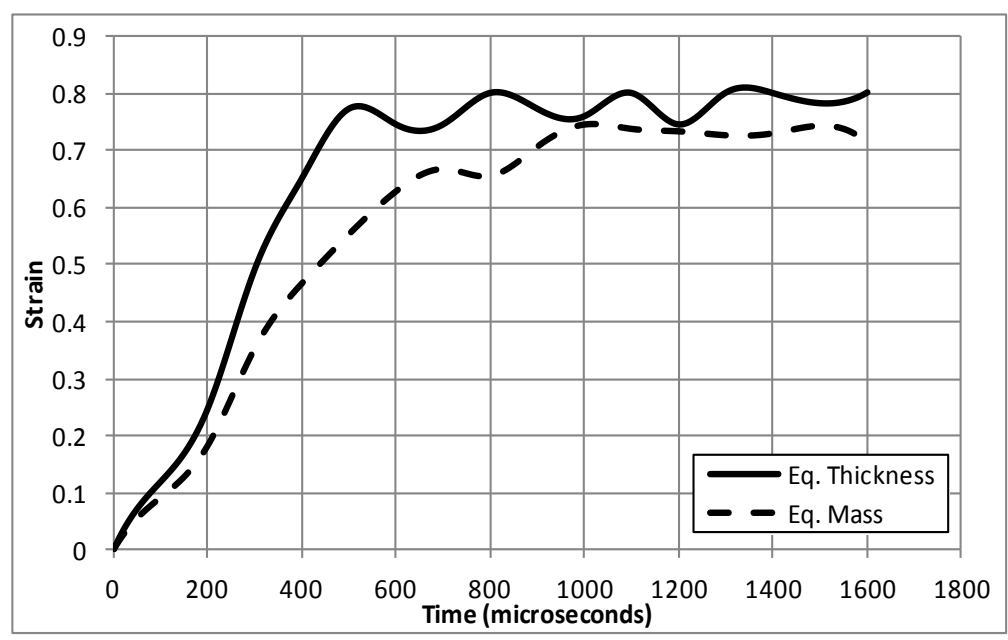

Fig. 12 Strain of A300 foam core layer along the mid-line (line of symmetry) for both configurations

\subsubsection{Digital Image Correlation (DIC) Analysis}

Utilizing the Digital Image Correlation (DIC) technique, the full-field deflection (W), in-plane strain $\left(\varepsilon_{\mathrm{yy}}\right)$ and particle velocity $(\mathrm{dW} / \mathrm{dt})$ of the back facesheet of each configuration were generated. Fig. 13 - Fig. 15 shows the full-field results for the back facesheet of both core arrangements respectively.

Fig. 13 shows the full-field out-of-plane deflection (W) for both configurations. Note the scale ranges from $0 \mathrm{~mm}$ (purple) to $42 \mathrm{~mm}$ (red). It is evident from the figure that the back face of both core configurations exhibits limited out-of-plane deflection prior to $\mathrm{t}=400 \mu \mathrm{s}$. Between $\mathrm{t}=400 \mu \mathrm{s}$ and $\mathrm{t}=1600 \mu \mathrm{s}$, both of these configurations continue to bend and exhibit deflections. For the sandwich composite with equivalent 
core layer thickness, as shown in Fig. 13a, it can be observed that at $t=1600 \mu \mathrm{s}$, the central region of the panel has deflected approximately $33 \mathrm{~mm}$. When using sandwich composites with equivalent core layer mass (Fig. 13b), it can be seen that at $t=1600$ $\mu \mathrm{s}$, the central region of the panel has deflected approximately $41 \mathrm{~mm}$. Therefore, it can be concluded that when using a core configuration with equivalent layer thickness, the deflection across the central region of the back facesheet is reduced approximately $20 \%$.

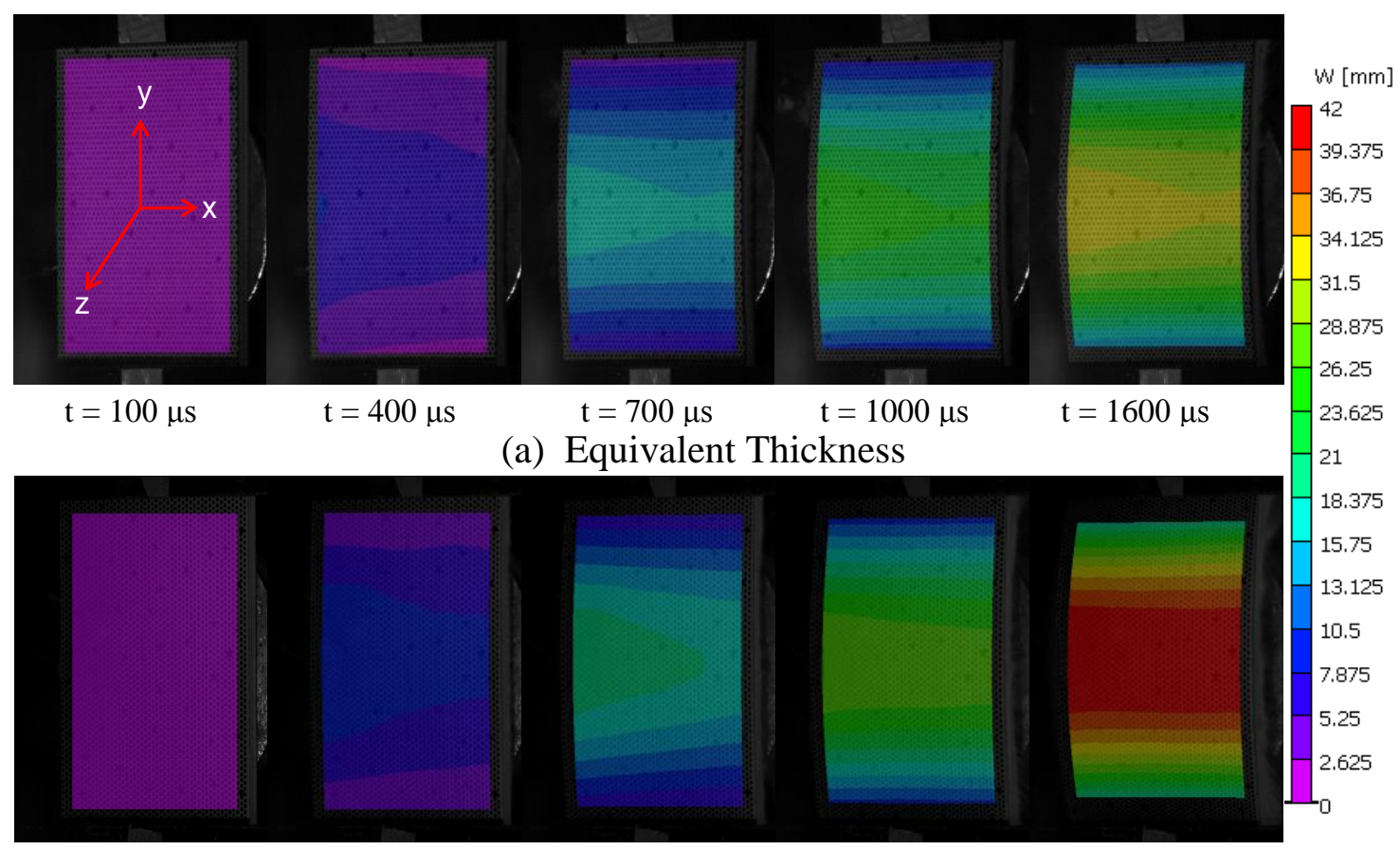

$\mathrm{t}=100 \mu \mathrm{s} \quad \mathrm{t}=400 \mu \mathrm{s}$ $\mathrm{t}=700 \mu \mathrm{s}$ $\mathrm{t}=1000 \mu \mathrm{s}$ $\mathrm{t}=1600 \mu \mathrm{s}$

(b) Equivalent Mass

Fig. 13 Full-field out-of-plane deflection (W) of both configurations

The full-field, in-plane strain $\left(\varepsilon_{\mathrm{yy}}\right)$ is shown in Fig 14 for both configurations with a scale of 0 (purple) to .025 (red), or $0 \%$ to $2.5 \%$ respectively. It can be observed in the figure that the back face of both core configurations exhibits very minimal in-plane strain $\left(\varepsilon_{\mathrm{yy}}\right)$ prior to $\mathrm{t}=100 \mu \mathrm{s}$. Between $\mathrm{t}=100 \mu \mathrm{s}$ and $\mathrm{t}=1600 \mu \mathrm{s}$, both of these configurations continue bending and the in-plane strain values continue to increase. 
For the sandwich composite with equivalent core layer thickness, as shown in Fig. $14 \mathrm{a}$, it can be observed that at $\mathrm{t}=1600 \mu \mathrm{s}$, the central region of the panel exhibits an in-plane strain of approximately .022 , or $2.2 \%$. When using sandwich composites with equivalent core layer mass (Fig. 14b), it can be seen that at $t=1600 \mu \mathrm{s}$, the central region of the panel exhibits an in-plane strain of approximately .024 , or $2.4 \%$. Therefore, it can be concluded that when using a core configuration with equivalent layer thickness, the maximum in-plane strain across the central region of the back facesheet reduced approximately $8 \%$.

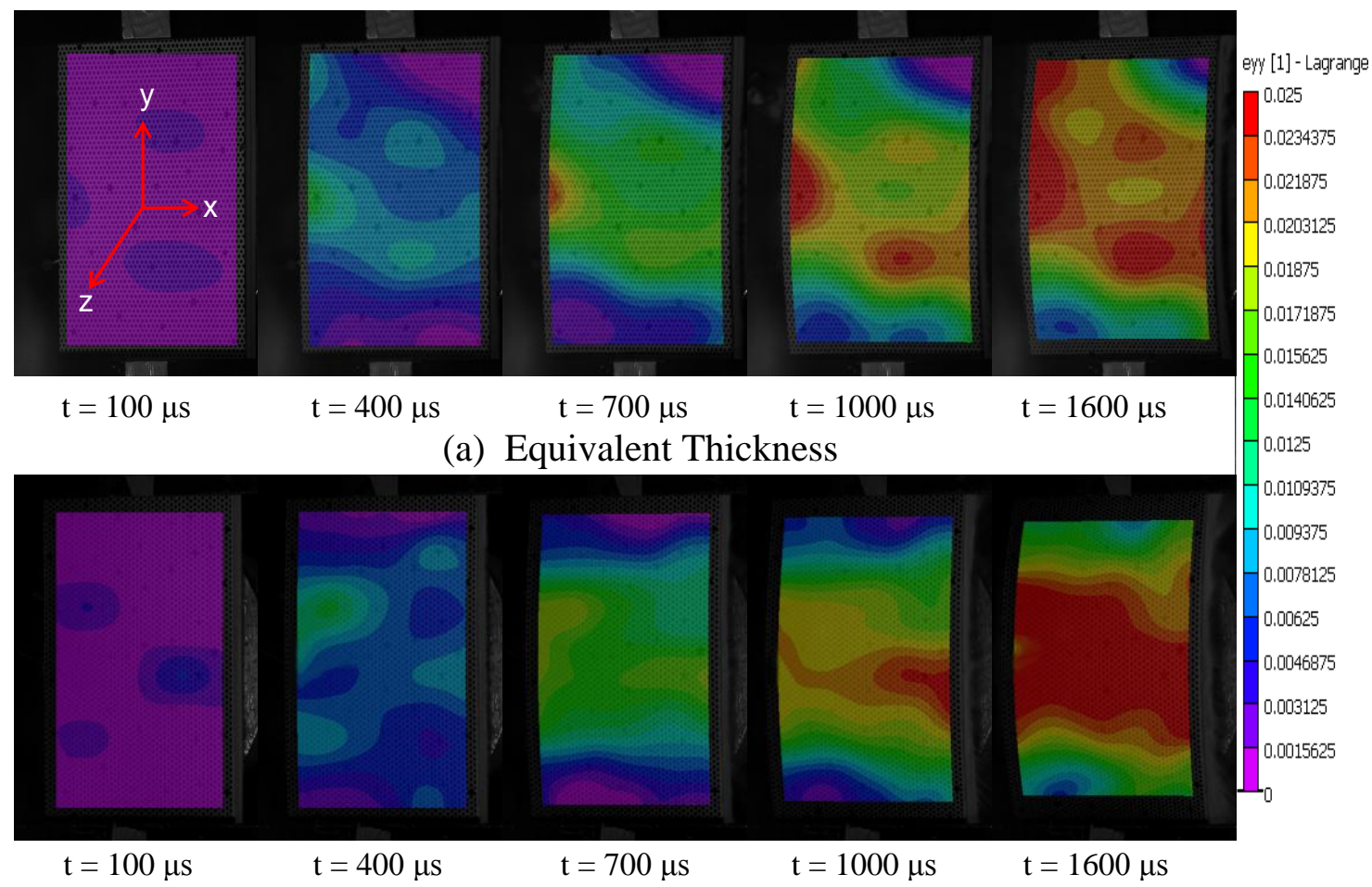

(b) Equivalent Mass

Fig. 14 Full-field in-plane strain $\left(\varepsilon_{\mathrm{yy}}\right)$ of both configurations

Fig. 15 shows the full-field out-of-plane velocity (dW/dt) for both configurations.

Note the scale ranges from $0 \mathrm{~mm} / \mathrm{s}$ (purple) to $35,000 \mathrm{~mm} / \mathrm{s}$ (red), or $0 \mathrm{~m} / \mathrm{s}$ to $35 \mathrm{~m} / \mathrm{s}$.

For the sandwich composites with equivalent core layer thickness, as shown in Fig. 15a, the back face exhibits a large out-of-plane velocity ( $25 \%$ of its maximum) by $\mathrm{t}$ 
$=100 \mu \mathrm{s}$. At $\mathrm{t}=400 \mu \mathrm{s}$, the central region of the back face has reached an out-of-plane velocity of approximately $30 \mathrm{~m} / \mathrm{s}$. By $\mathrm{t}=700 \mu \mathrm{s}$ the velocity has reached a maximum value of $31 \mathrm{~m} / \mathrm{s}$, and reduced back to approximately $30 \mathrm{~m} / \mathrm{s}$. From $\mathrm{t}=700 \mu \mathrm{s}$ and onward, the velocity continues to decrease from $30 \mathrm{~m} / \mathrm{s}$ to $22 \mathrm{~m} / \mathrm{s}(\mathrm{t}=1000 \mu \mathrm{s})$ and finally down to $7 \mathrm{~m} / \mathrm{s}(\mathrm{t}=1600 \mu \mathrm{s})$.

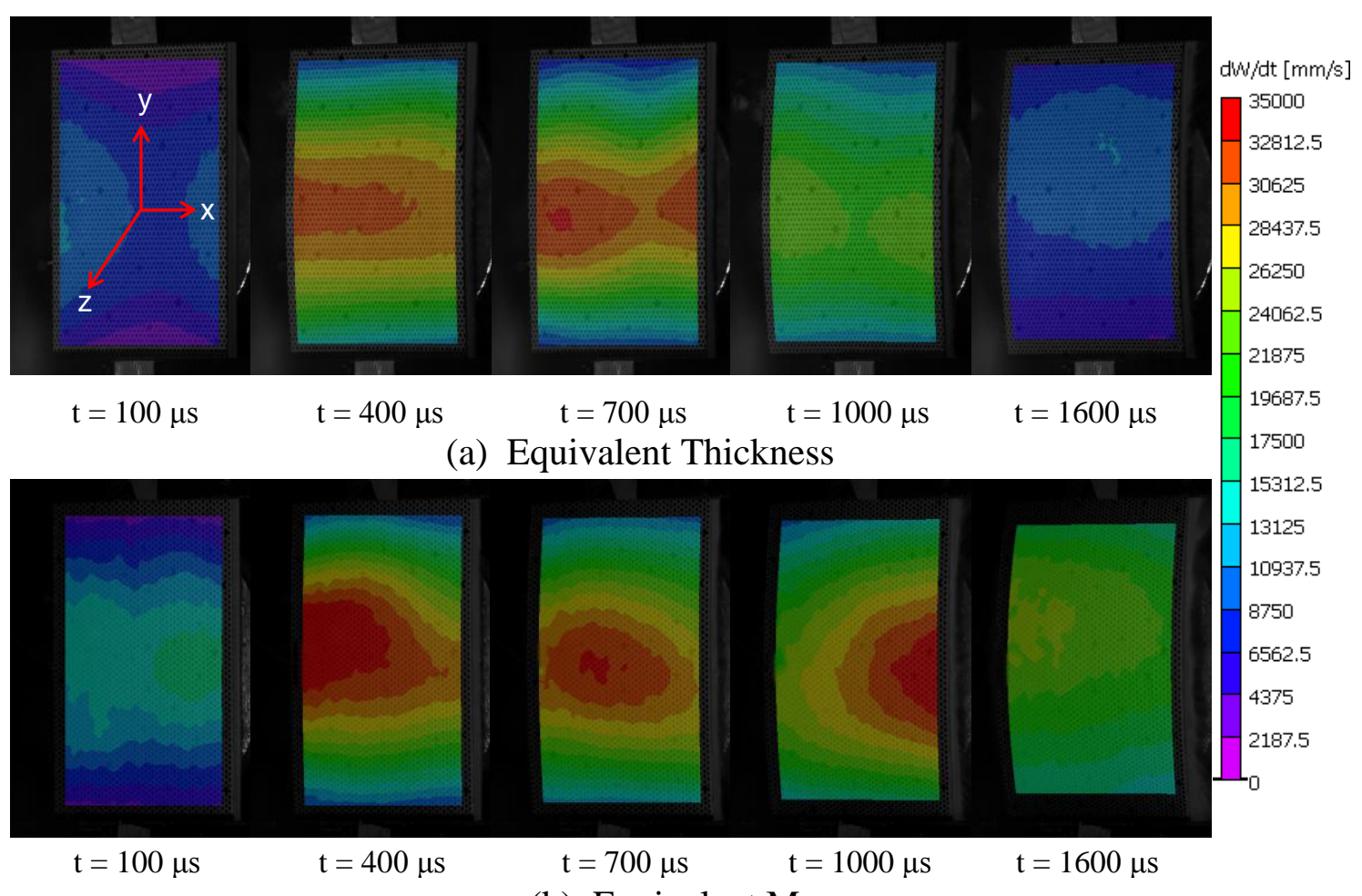

(b) Equivalent Mass

Fig. 15 Full-field out-of-plane velocity (dW/dt) of both configurations

For the sandwich composites with equivalent core layer mass, as shown in Fig. $15 \mathrm{~b}$, the back face exhibits a larger out-of-plane velocity ( $\sim 40 \%$ of its maximum) by $\mathrm{t}$ $=100 \mu \mathrm{s}$. At $\mathrm{t}=400 \mu \mathrm{s}$, the central region of the back face has reached an out-of-plane velocity of approximately $31 \mathrm{~m} / \mathrm{s}$. By $\mathrm{t}=700 \mu \mathrm{s}$ the velocity has reached a maximum values, $34 \mathrm{~m} / \mathrm{s}$, and reduced to $33 \mathrm{~m} / \mathrm{s}$. From $\mathrm{t}=700 \mu \mathrm{s}$ and onward, the velocity continues to decrease from $33 \mathrm{~m} / \mathrm{s}$ to $29 \mathrm{~m} / \mathrm{s}(\mathrm{t}=1000 \mu \mathrm{s})$ and finally down to $20 \mathrm{~m} / \mathrm{s}$ $(\mathrm{t}=1600 \mu \mathrm{s})$. 
Therefore, it can be concluded that when using a sandwich composites with equivalent core layer thickness, the maximum back face velocity is approximately $8 \%$ smaller than the sandwich composite with equivalent core layer mass. Also note, the velocity of the panel at $\mathrm{t}=1600 \mu \mathrm{s}$ is approximately $65 \%$ smaller as well, i.e. $20 \mathrm{~m} / \mathrm{s}$ and $7 \mathrm{~m} / \mathrm{s}$ respectively.

Using the point inspection tool from the Digital Image Correlation (DIC) software, a point directly in the center of the back face of each specimen was chosen. The out-of-plane deflection (W) showed excellent agreement with the results generated utilizing the high-speed images and therefore, only the in-plane strain $\left(\varepsilon_{y y}\right)$ and out-of-plane velocity (dW/dt) results are shown. Fig. 16 and Fig. 17 show the inplane strain and out-of-plane velocity values obtained. Looking at the in-plane strain values (Fig. 16) it can be seen that at $t=1600 \mu \mathrm{s}$, the maximum in-plane strain value at the central point of the back facesheet for the sandwich composite with equivalent core layer thickness is approximately 0.0225 , or $2.25 \%$. When using a sandwich composite with equivalent core layer mass, it can be seen that at $t=1600 \mu$ s, the maximum in-plane strain value at the central point of the back facesheet is approximately 0.0245 , or $2.45 \%$. Therefore, it can be concluded that when using sandwich composites with equivalent core layer thickness, the maximum in-plane strain across the central region of the back facesheet is reduced $8 \%$ in comparison to the sandwich composites with equivalent core layer mass. 


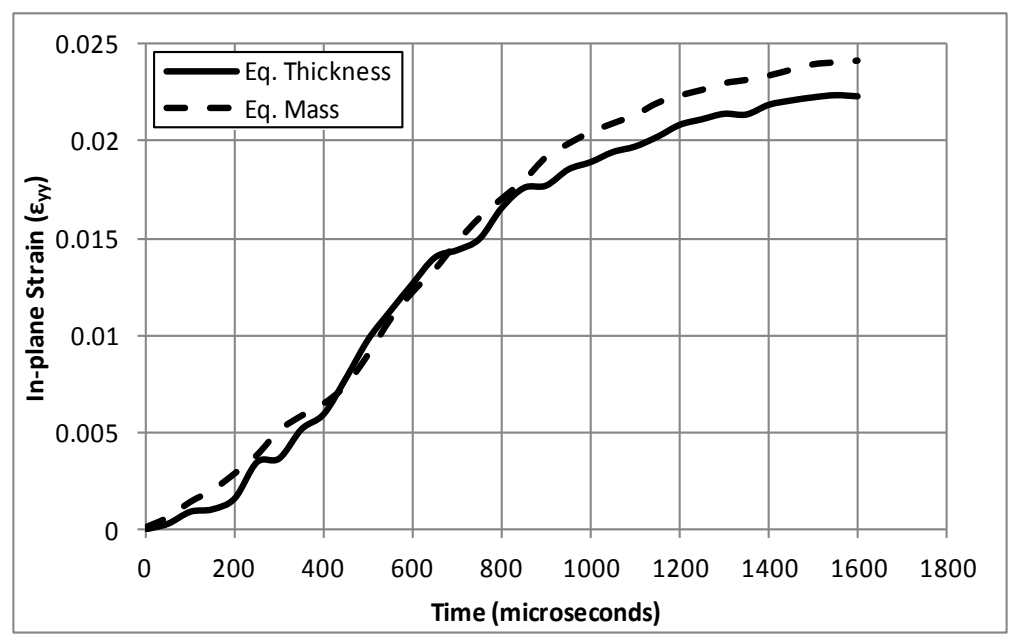

Fig. 16 In-plane strain $\left(\varepsilon_{y y}\right)$ of both configurations

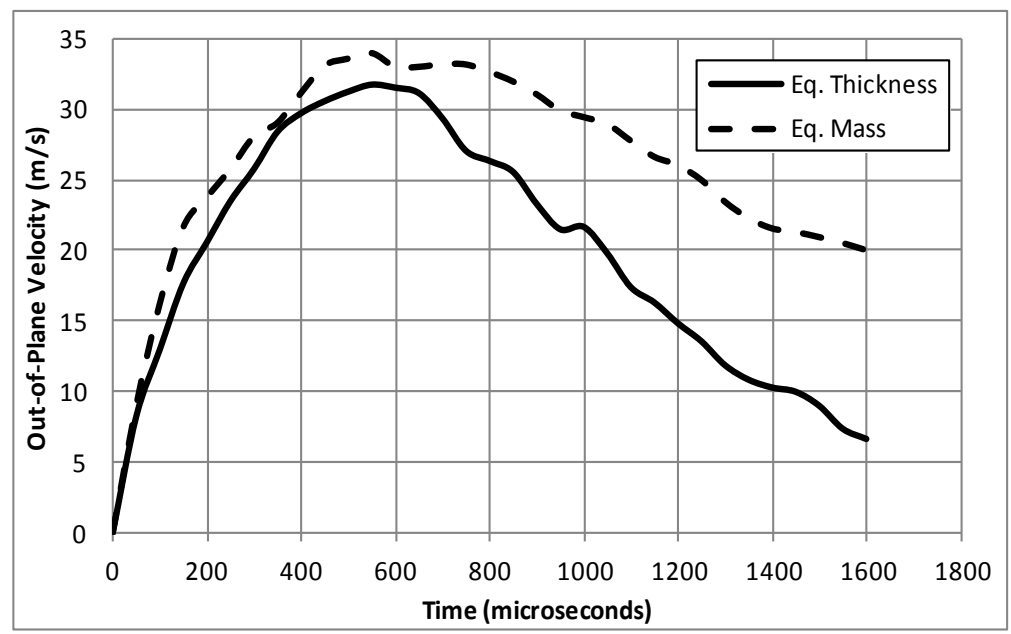

Fig. 17 Out-of-plane velocity (dW/dt) of both configurations

Fig. 17 shows the out-of-plane velocity (dW/dt) for both configurations. For the sandwich composite with equivalent core layer thickness, the maximum velocity is reached at $\mathrm{t}=500 \mu \mathrm{s}$ and is approximately $31 \mathrm{~m} / \mathrm{s}$. For the sandwich composite with equivalent core layer mass, the maximum velocity is reached at approximately the same time, $\mathrm{t}=500 \mu \mathrm{s}$, but the velocity is larger $(34 \mathrm{~m} / \mathrm{s})$. Therefore, it can be concluded that when using sandwich composites with equivalent core layer thickness, the maximum out-of-plane velocity across the central region of the back facesheet is 
reduced $8 \%$ in comparison to the sandwich composites with equivalent core layer mass.

\subsubsection{Post-mortem Analysis}

After the blast loading event occurred, the damage patterns of the sandwich composites with both core layer arrangements were visually examined and recorded using a high resolution digital camera and are shown in Fig.18. Note the locations and damage mechanisms were identical for both configurations; the only difference was in the extent of damage observed. When the sandwich composite with equivalent core layer thickness was subjected to highly-transient loading, as shown in Fig. 18a, the damage was confined to the areas where the supports were located in the shock tube and core cracking is visible in these two areas. The core cracks propagated completely through the foam core. Core delamination is visible between the front facesheet and the foam core. Also, the core cracks lead to back skin delamination, where the core separated from the back facesheet. Some core compression is also visible in the first core layer (A300) of foam.

For the sandwich composite with equivalent core layer mass, the damage patterns after being subjected to the shock loading are shown in Fig. 18b. For this core configuration, the damage was again confined to the areas where the supports were located in the shock tube and core cracking is evident. The core cracks propagated completely through the foam core. Skin delamination is obvious between the front facesheet and the foam core, as well as back skin delamination between the back facesheet and the foam core. Also, heavy front face fiber delamination and core compression in the first layer of foam (A300) can be observed. 
Front facesheet

(Blast-receiving side)

Foam Core Back facesheet

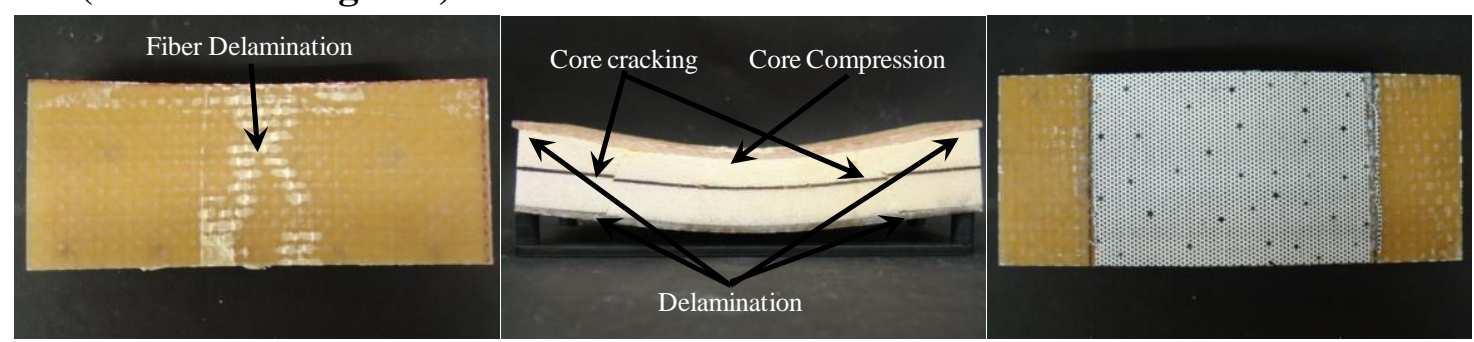

(a) Equivalent Thickness

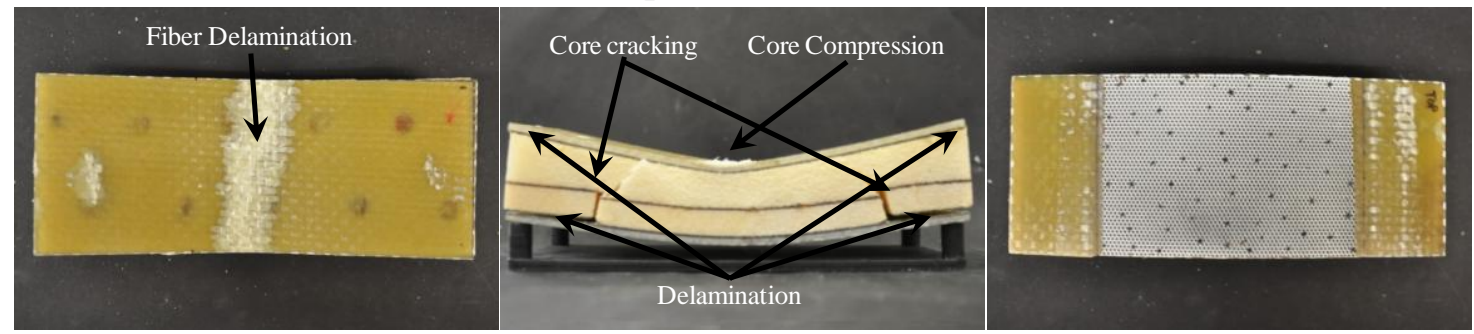

(b) Equivalent Mass

Fig. 18 Visual examination of both configurations after being subjected to high intensity blast load

\subsubsection{Energy Redistribution Behavior}

The energy redistribution behavior of both configurations was next analyzed using the methods described by Wang et al. [27]. The total energy loss and the total deformation energy of the sandwich composites with equivalent core layer thickness and equivalent core layer mass, as calculated during the blast loading event are shown in Fig. 19. Total energy loss is characterized as the difference between the incident and remaining energies of the gas and total deformation energy is defined as the work done by the gas to deform the specimen. This total energy loss is consumed in panel deformation energy, panel kinetic energy, heat, sound, light and any energy lost out of the side of the panel during bending. 


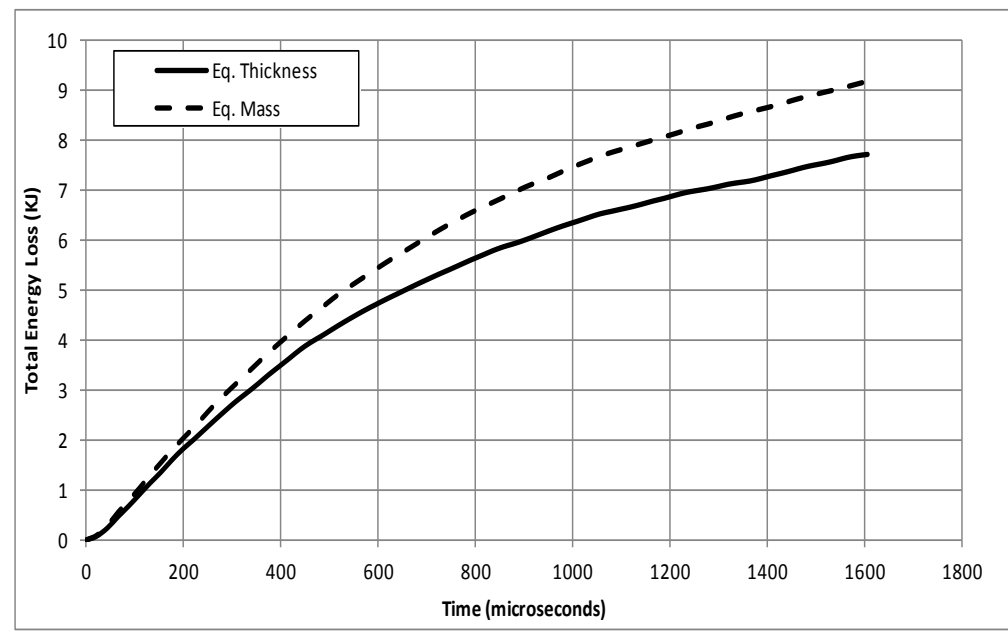

(a) Total energy loss in both configurations during the blast loading event

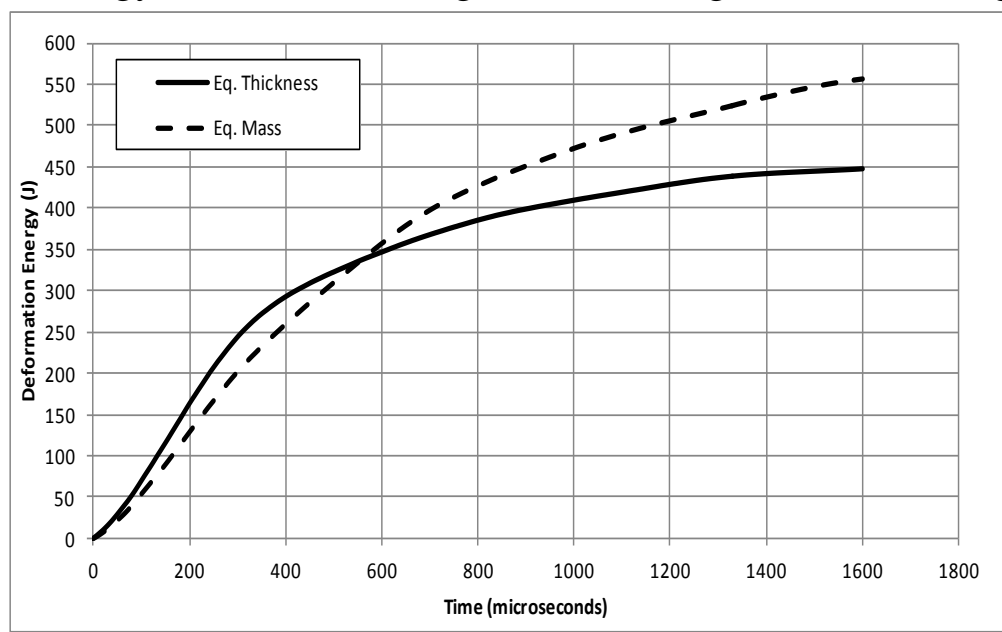

(b) Deformation energy of both configurations during the blast loading event

Fig. 19 Energy redistribution behavior of both configurations during the blast loading event

It can be observed in Fig. 19a and Fig. 19b that at $t=1800 \mu \mathrm{s}$, the total energy loss and the deformation energy of the sandwich composite with equivalent core layer mass are approximately $20 \%$ and $15 \%$ more than that of the sandwich composites with equivalent core layer thickness. This indicates that using equivalent core layer mass, thus increasing the thickness of the A300 (softer) foam core layer allows for more energy to be consumed during the shock loading process, resulting in heavier core damage as observed in Fig. 18b. Also note that a very small amount of energy, 
approximately $5 \%$ of the total energy loss, is used to deform the panel, and the bulk of the energy is lost elsewhere.

Using Matlab, seven points were chosen (between the simple-supports) along the profile of the front face of the panel using the high-speed images (Fig. 9). The splinecurve fitting method was utilized to track the deformation of the front face throughout the shock wave loading event. The reconstructed shapes, as generated by the seven data points, are shown in Fig. 20. Since the deformation energy of both configurations (Fig. 19b) is very similar prior to $t=600 \mu$ s, the reconstructed shapes of the front face of both configurations was carried out to include this time (up to approximately $\mathrm{t}=$ $800 \mu \mathrm{s})$. Through this reconstruction method, the deflection data of each point of the front face of each panel (such as Fig. 20a) can be obtained and utilized in the calculation of the deformation energy (Fig. 19b). Therefore, it can be observed that the shape of the front face of the panel with equivalent core layer mass exhibits a sharper bending profile in comparison to the panel with equivalent core layer thickness. Due to the increased thickness of the A300 (softer) foam core layer and the decreased thickness of the A800 (stiffer layer), this configuration (equivalent core layer mass) results in a core which allows for more localized compression (muzzle diameter) and a larger amount of bending. This in turn results in more gas escaping out the sides and more damage during deformation, which lowers the reflected pressures and allows for a higher total energy loss. 


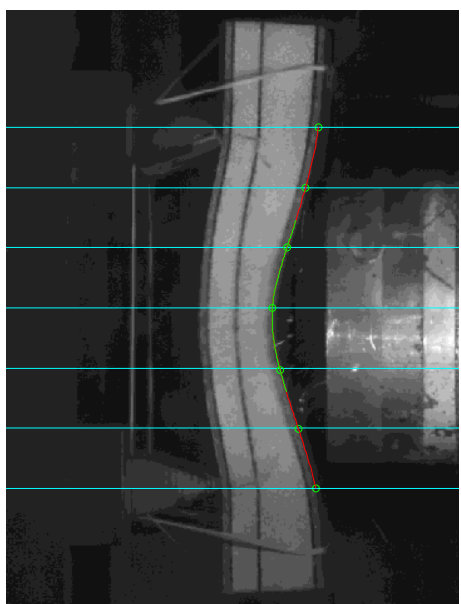

(a) Spline curve fit on front face of sandwich composite with eq. core layer mass

$$
(\mathrm{t}=400 \mu \mathrm{s})
$$

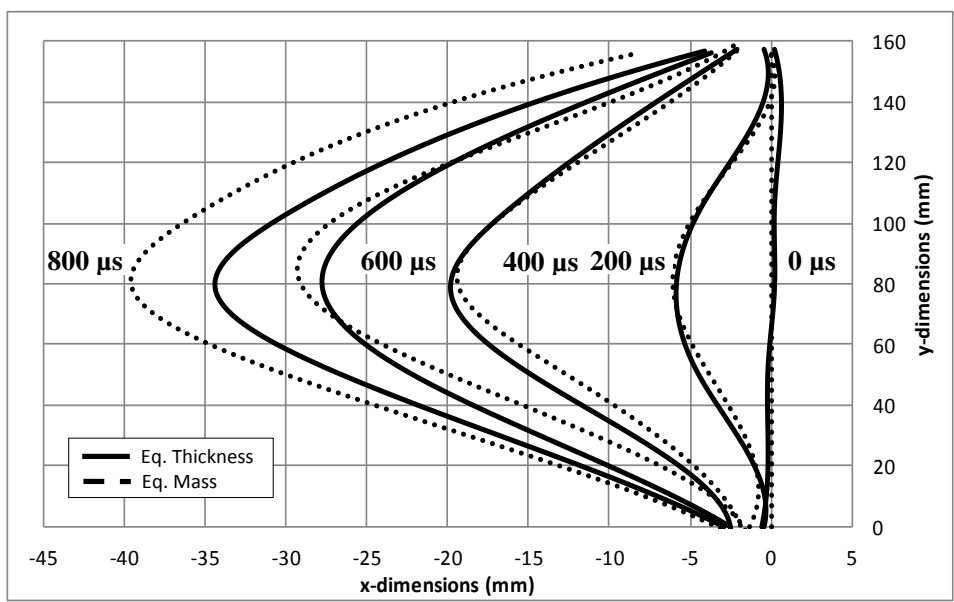

(b) Reconstructed shape of front face of both configurations

(spline curve fit between support fixture-red line)

Fig. 20 Shape reconstruction analysis of both configurations

\subsubsection{Bending Stiffness and Strength of Sandwich Beams}

In order to achieve a better understanding of the performance of these sandwich panels and the phenomena observed, the bending stiffness (flexural rigidity) and strength of each configuration will be calculated and compared. For simplicity the shock loading event, which utilizes a beam (specimen), held in simple-supports, will be modeled as a sandwich beam centrally loaded under three-point bending (quasistatic). This type of analysis on the bending stiffness and strength of sandwich beams has been extensively investigated by [28-34]. Note this general theory is based on the shear deformable beams theory and has been discussed in detail by Allen [28]. This theory uses the assumption of uniform deflection and a linear longitudinal displacement field in the core through its thickness. Also, the facesheets and foam core materials are assumed to be homogeneous and isotropic. 

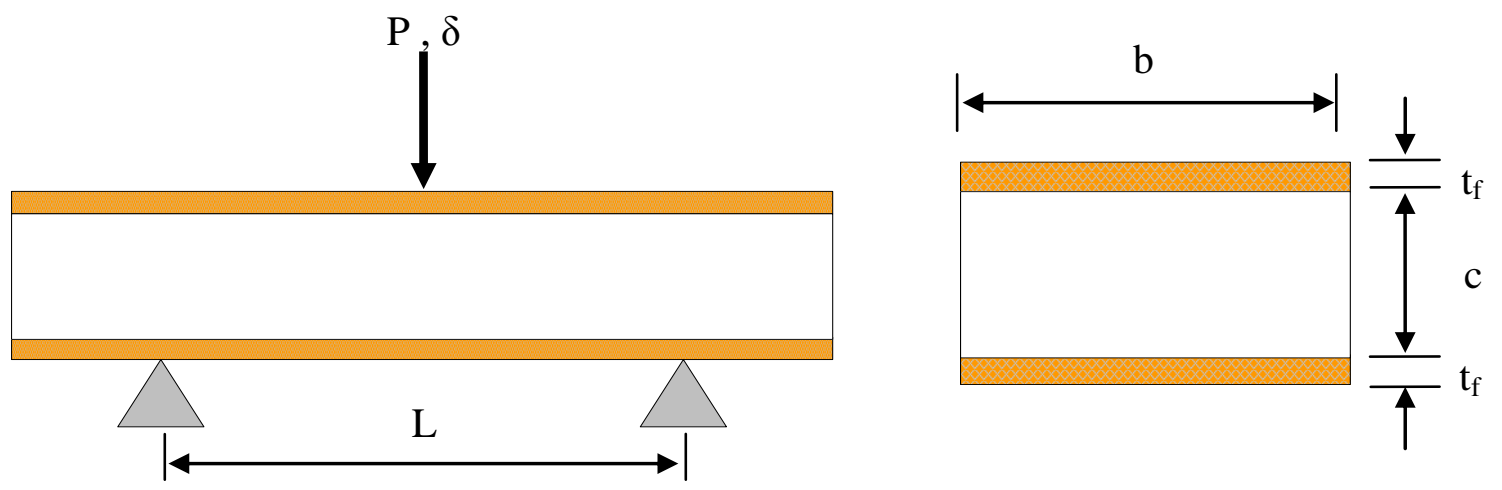

Fig. 21 Simply supported sandwich beam centrally loaded in three-point bending
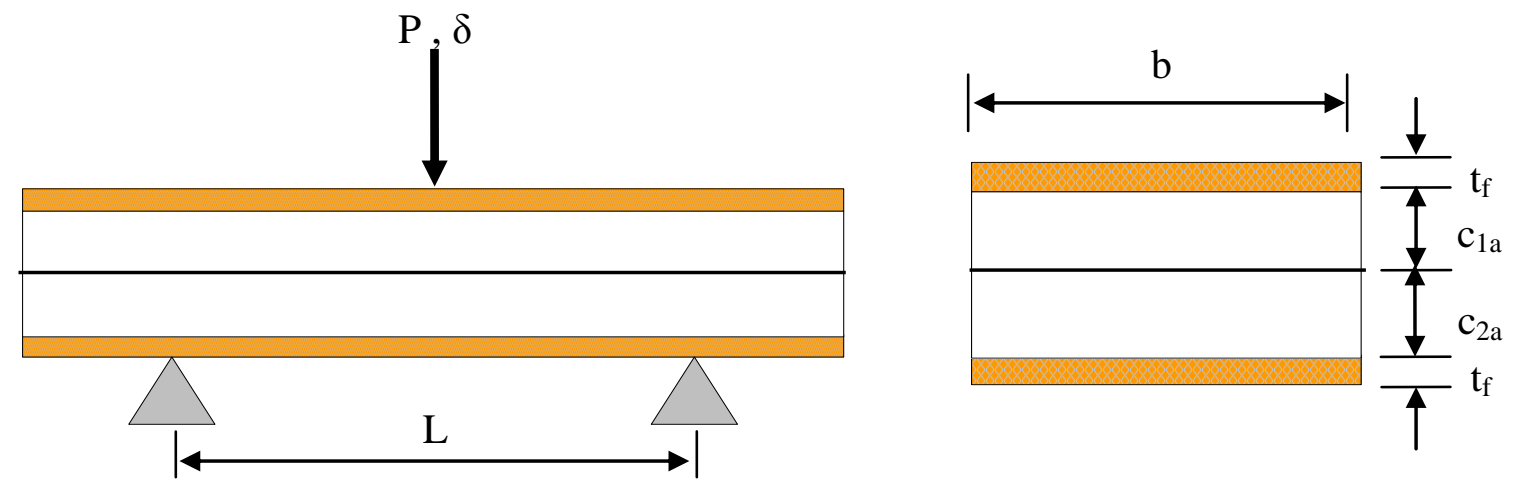

(a) Sandwich composite with equivalent core layer thickness
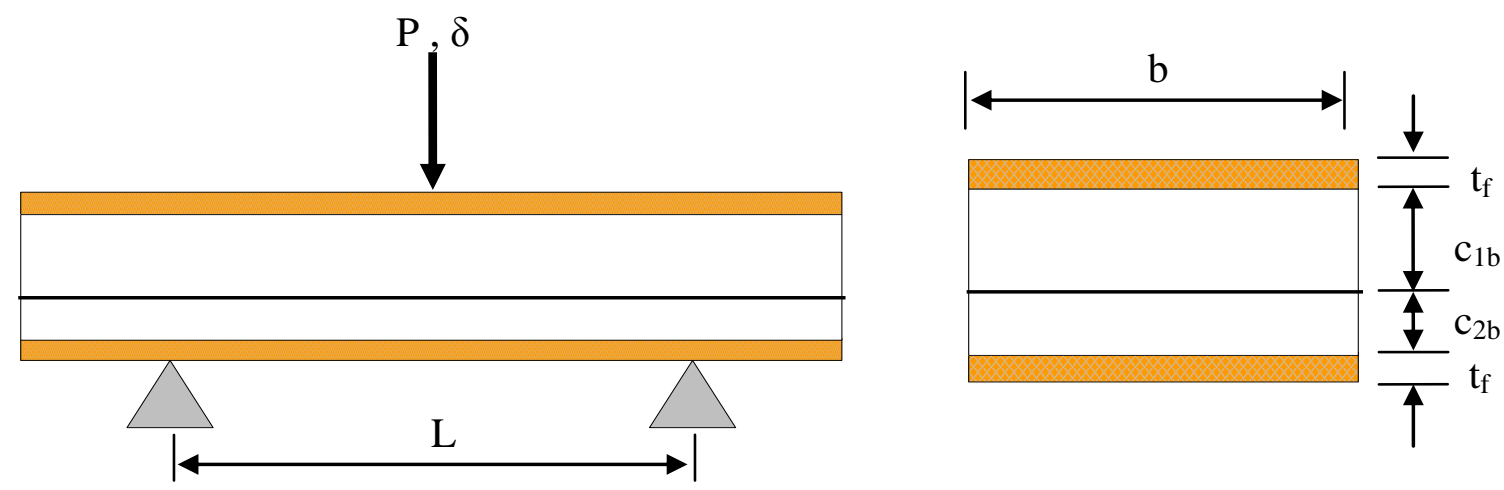

(b) Sandwich composite with equivalent core layer mass

Fig. 22 Modified simply supported sandwich beam centrally loaded in three-point bending

Consider a simply supported sandwich beam centrally loaded in three-point bending, as shown in Fig. 21. The mid-point of the beam deflects by a transverse displacement, $\delta$, due to the applied transverse load, P. Let L be the beam length between the supports, $b$ the width of the beam, $c$ the core thickness, and $t_{f}$ the face 
thickness. The relevant material properties of the facesheets are the transverse compressive strength, $\sigma_{\mathrm{f}}$, and Young's modulus, $\mathrm{E}_{\mathrm{f}}$; for the core, the relevant properties are the compressive Young's modulus, $\mathrm{E}_{\mathfrak{c}}$, shear modulus, $\mathrm{G}_{\mathrm{c}}$, and shear strength, $\tau_{\mathrm{c}}$.

Table 3. Specimen dimensions and three-point bending schematic variables (Fig. 22)

\begin{tabular}{|c|c|}
\hline \multicolumn{2}{|c|}{ Variables } \\
\hline $\mathbf{P}$ & $\mathrm{P}$ \\
$\mathbf{L}$ & $0.1524 \mathrm{~m}$ \\
$\mathbf{E}_{\mathbf{f}}$ & $3.9 \mathrm{MPa}$ \\
$\mathbf{b}$ & $.1016 \mathrm{~m}$ \\
$\mathbf{t}_{\mathbf{f}}$ & $0.005 \mathrm{~mm}$ \\
$\mathbf{d}$ & $0.043 \mathrm{~mm}$ \\
$\mathbf{E}_{\mathbf{c 1}}$ & $32 \mathrm{MPa}$ \\
$\mathbf{E}_{\mathbf{c 2}}$ & $117 \mathrm{MPa}$ \\
$\mathbf{c}_{\mathbf{1 a}}$ & $0.019 \mathrm{~m}$ \\
$\mathbf{c}_{\mathbf{2 a}}$ & $0.019 \mathrm{~m}$ \\
$\mathbf{c}_{\mathbf{1 b}}$ & $0.0254 \mathrm{~m}$ \\
$\mathbf{c}_{\mathbf{2 b}}$ & $0.0127 \mathrm{~m}$ \\
$\mathbf{G}_{\mathbf{c} 1}$ & $20 \mathrm{MPa}$ \\
$\mathbf{G}_{\mathbf{c} 2}$ & $47 \mathrm{MPa}$ \\
$\boldsymbol{\tau}_{\mathbf{c 1}}$ & $0.6 \mathrm{MPa}$ \\
$\boldsymbol{\tau}_{\mathbf{c 2}}$ & $1.6 \mathrm{MPa}$ \\
$\boldsymbol{\sigma}_{\mathbf{f}}$ & $700 \mathrm{MPa}$ \\
\hline
\end{tabular}

Allen [28] gives the total deflection $\delta$ at the mid-point of a sandwich beam loaded in three-point bending as the sum of the deflections due to bending of the facesheets and the shear of the core:

$$
\delta=\frac{P L^{3}}{48(E I)_{e q}}+\frac{P L}{4(A G)_{e q}}
$$

where $(E I)_{\text {eq }}$ is the equivalent flexural rigidity 


$$
(E I)_{e q}=\frac{E_{f} b t_{f} d^{2}}{2}+\frac{E_{f} b t_{f}^{3}}{6}+\frac{E_{c} b c^{3}}{12}
$$

and $(\mathrm{AG})_{\mathrm{eq}}$ is the equivalent shear rigidity

$$
(A G)_{e q}=\frac{b d^{2} G_{c}}{c} \approx b d G_{c}
$$

in terms of the geometric parameters defined above, and the distance between the centroids of the faces $d=c+t_{f}$

Table 4. Comparison of quasi-static flexural and shear rigidity of both configurations

\begin{tabular}{|c|c|c|c|c|}
\hline & Units & Equivalent Thickness & Equivalent Mass & \% difference \\
\hline$(\mathbf{E I})_{\mathbf{e q}}$ & $\mathbf{N ~} \mathbf{~ m ~ m}^{\mathbf{2}}$ & 6.45 & 5.28 & $18 \%$ \\
$(\mathbf{A G})_{\mathbf{e q}}$ & $\mathbf{K N}$ & 104.33 & 70.30 & $33 \%$ \\
Deflection, $\boldsymbol{\delta}$ & $\mathbf{m}[\mathbf{f}(\mathbf{P})]$ & $1.17 \mathrm{E}-05 \times \mathrm{P}$ & $1.43 \mathrm{E}-05 \times \mathrm{P}$ & $18 \%$ \\
\hline
\end{tabular}

Table 4 summarizes the results as obtained using Eq. (13)- Eq. (15) coupled with the corresponding material properties of the given facesheet and foam core material (Table 1), as well as the specimen dimensions (Table 3) and loading conditions (Fig. 22). Note, when applying the above equations to sandwich composites with more than one core layer, the Young's modulus and shear modulus of the foam core, $\mathrm{E}_{\mathrm{c}}$ and $\mathrm{G}_{\mathrm{c}}$ respectively, were calculated using a weighted average, i.e.,

$$
\begin{gathered}
E_{c a}=\frac{\left(E_{c 1}\right)\left(c_{1 a}\right)+E_{c 2}\left(c_{2 a}\right)}{c_{1 a}+c_{2 a}}, \quad E_{c b}=\frac{\left(E_{c 1}\right)\left(c_{1 b}\right)+E_{c 2}\left(c_{2 b}\right)}{c_{1 b}+c_{2 b}} \\
G_{c a}=\frac{\left(G_{c 1}\right)\left(c_{1 a}\right)+G_{c 2}\left(c_{2 a}\right)}{c_{1 a}+c_{2 a}}, \quad G_{c b}=\frac{\left(G_{c 1}\right)\left(c_{1 b}\right)+G_{c 2}\left(c_{2 b}\right)}{c_{1 b}+c_{2 b}}
\end{gathered}
$$

where $c_{1}$ and $c_{2}$ are the respective core thicknesses of A300 and A800 for the sandwich composites with equivalent core layer thickness (denoted with subscript a) and equivalent core layer mass (denoted with subscript $b$ ). These weighted averages 
were used in Eq. (14) and Eq. (15) respectively. Also note, the load P was left as a variable, since the same load was applied to both configurations.

Therefore, it can be observed in Table 4, that the sandwich composites with equivalent core layer mass, thus a thicker A300 (softer) foam core layer and a thinner A800 (stiffer) foam core layer, the equivalent flexural rigidity $\left(\mathrm{EI}_{\mathrm{eq}}\right)$ and shear rigidity $\left(\mathrm{AG}_{\mathrm{eq}}\right)$ are reduced $18 \%$ and $33 \%$ respectively. This results in a quasi-static deflection under three-point bending that is $18 \%$ larger for the sandwich composite with equivalent core layer mass than the sandwich composite with equivalent core layer thickness. These quasi-static results can be extended to the dynamic performance of the sandwich panel with equivalent core layer mass under shock wave loading, and it can be seen that a higher back face deflection, in-plane-stain and velocity, as described in section 4.2.3 (Digital Image Correlation (DIC) Analysis) was achieved.

Foam core composite sandwich beams under quasi-static three-point and fourpoint bending loads can fail under four main modes: face yielding or microbuckling, face wrinkling, core shear yield and core compressive yield (indentation), [28,31,32]]. For a sandwich beam in three-point bending, as shown in Fig.21, the predicted collapse loads are as follows:

For face yielding or microbuckling,

$$
P=\frac{4 b d t_{f} \sigma_{f}}{L}
$$

For face wrinkling,

$$
P=\frac{2 b t_{f} d}{L}+\sqrt[3]{E_{f} E_{c} G_{c}}
$$


note, this expression includes a knockdown factor of almost two (2) associated with the assumed geometrical imperfections of the facesheet [28].

For core shear failure,

$$
\begin{gathered}
P=2 b d \tau_{c} \\
P=2 b d \tau_{c}+8 E_{f} b\left(\frac{t_{f}}{L}\right)^{3} \delta
\end{gathered}
$$

where eq. (18(a)) results in the same yield load as eq. (18(b)) but includes a postyield hardening response with a slope controlled by the bending stiffness of the facesheets [33].

For indentation failure (core compressive yield) [30],

$$
P=b t_{f}\left(\frac{\pi^{2} \sigma_{c}^{2} E_{f} d}{3 L}\right)^{1 / 3}
$$

which assumes that the compressive sandwich face behaves as an elastic beam-column with the core as a rigid-ideally plastic foundation.

For the purposes of this investigation, only face yielding, core shear and indentation (core compressive yield) failure loads will be investigated and compared. Table 5 summarizes the results as obtained using Eq. (16)- Eq. (19) coupled with the corresponding material properties of the given facesheet and foam core material (Table 1), as well as the specimen dimensions (Table 3) and loading conditions (Fig. 22). Note, when applying the above equations, to sandwich composites with more than one core layer, the shear strength and the compressive strength of the foam core, $\tau_{\mathrm{c}}$ and $\sigma_{\mathrm{c}}$ respectively, were calculated again using a weighted average, i.e.,

$$
\tau_{c a}=\frac{\left(\tau_{c 1}\right)\left(c_{1 a}\right)+\tau_{c 2}\left(c_{2 a}\right)}{c_{1 a}+c_{2 a}}, \quad \tau_{c b}=\frac{\left(\tau_{c 1}\right)\left(c_{1 b}\right)+\tau_{c 2}\left(c_{2 b}\right)}{c_{1 b}+c_{2 b}}
$$




$$
\sigma_{c a}=\frac{\left(\sigma_{c 1}\right)\left(c_{1 a}\right)+\tau_{c 2}\left(\sigma_{2 a}\right)}{c_{1 a}+c_{2 a}}, \quad \sigma_{c a}=\frac{\left(\sigma_{c 1}\right)\left(c_{1 b}\right)+\sigma_{c 2}\left(c_{2 b}\right)}{c_{1 b}+c_{2 b}}
$$

where $c_{1}$ and $c_{2}$ are the respective core thicknesses of $A 300$ and A800 for the sandwich composites with equivalent core layer thickness (denoted with subscript a) and equivalent core layer mass (denoted with subscript b). These weighted averages were used in Eq. (18) and Eq. (19) respectively. Also note, the deflection, $\delta$, was left as a variable, since it's a function of the applied load, $\mathrm{P}$.

Table 5. Comparison of different quasi-static three-point bending failure modes and

\begin{tabular}{|c|c|c|c|c|}
\hline \multirow{2}{*}{ Failure Modes } & \multirow{2}{*}{ units } & \multicolumn{2}{|c|}{ Failure Load } & \multirow{2}{*}{$\begin{array}{c}\% \\
\text { difference }\end{array}$} \\
\hline & & Eq. Thickness & Eq. Mass & \\
\hline $\begin{array}{l}\text { Face yield or } \\
\text { microbuckling }\end{array}$ & $\mathbf{K N}$ & \multicolumn{2}{|c|}{401.3} & ------ \\
\hline Core Shear, Eq. (18a) & $\mathbf{K N}[(\mathbf{f}(\boldsymbol{\delta})]$ & $9.61 \times \delta$ & $8.11 \times \delta$ & $16 \%$ \\
\hline Core Shear, Eq. (18b) & $\mathbf{K N}[\mathbf{f}(\boldsymbol{\delta})]$ & $9.72 \times \delta$ & $8.25 \times \delta$ & $15 \%$ \\
\hline $\begin{array}{c}\text { Indentation } \\
\text { (core compressive yield) }\end{array}$ & $\mathbf{N}$ & 929.11 & 795.81 & $14 \%$ \\
\hline
\end{tabular}

Therefore, it can be observed in Table 5, that the sandwich composites with equivalent core layer mass, thus a thicker A300 (softer) foam core layer and a thinner A800 (stiffer) foam core layer, failure modes occur at a load which is approximately $15 \%$ less than that of the sandwich composites with equivalent core layer thickness. This results in panel which exhibits more damage, as described in section 4.2.4 (Postmortem analysis).

\section{Conclusions}

The following is a summary of this investigation:

(1) The dynamic stress-strain response is significantly higher than the quasi-static response for the two type of Corecell ${ }^{\mathrm{TM}}$ A-series foam studied. Both quasi- 
static and dynamic constitutive behaviors of Corecell ${ }^{\mathrm{TM}}$ A series foams (A300 and A800) show an increasing trend. The increase in the yield strength from quasi-static response to dynamic response, along with the longer stress plateau, indicates that these foam materials show great potential in absorbing large amounts of energy.

(2) Sandwich composites with two types of monotonically graded cores based on increasing wave impedance were subjected to blast loading. In order to reduce areal density, a sandwich composite with equivalent core layer mass was fabricated and its blast performance was compared to its sandwich composite counterpart with equivalent core layer thickness. Table 6 lists the overall results of this investigation.

(3) The flexural stiffness and shear rigidity were numerically investigated to achieve a better understanding on the overall behavior of the two types of sandwich composites. It was observed that utilizing a sandwich composite with equivalent core layer mass, thus increasing the thickness of the A300 (softer) layer, decreasing the thickness of the A800 (stiffer) layer and reducing the overall areal density, results in specimen whose stiffness and strength is significantly lower $(\sim 20 \%)$ in comparison to the sandwich specimen with equivalent core layer thickness. 
Table 6. Overall comparison of final results

\begin{tabular}{|c|c|c|c|c|}
\hline & Units & $\begin{array}{c}\text { Equivalent } \\
\text { Thickness }\end{array}$ & $\begin{array}{c}\text { Equivalent } \\
\text { Mass }\end{array}$ & Difference \\
\hline Areal Density & $\mathrm{Kg} / \mathrm{m}^{2}$ & 18.5 & 17.6 & $\downarrow \mathbf{5 \%}$ \\
Deflection & $\mathrm{mm}$ & 33 & 42 & $\uparrow \mathbf{2 1 \%}$ \\
$\begin{array}{c}\text { In-plane Strain } \\
\text { Out-of-plane } \\
\text { Velocity } \\
\text { Total Energy } \\
\begin{array}{c}\text { Loss } \\
\text { Deformation } \\
\text { Energy }\end{array}\end{array}$ & $\mathrm{m} / \mathrm{s}$ & 31 & 2.46 & $\uparrow \mathbf{8 \%}$ \\
$\begin{array}{c}\text { (EI) } \\
\text { eq }\end{array}$ & $\mathrm{J}$ & 7.7 & 9.2 & $\uparrow \mathbf{1 6 \%}$ \\
& $\mathrm{N} \mathrm{x} \mathrm{m} \mathrm{m}^{2}$ & 6.5 & 5.3 & $\downarrow \mathbf{1 8 \%}$ \\
\hline
\end{tabular}

\section{Acknowledgements}

The authors kindly acknowledge the financial support provided by Dr. Yapa D. S. Rajapakse, under Office of Naval Research (ONR) Grant No. N00014-10-1-0662. The authors acknowledge the support provided by the Department of Homeland Security (DHS) under Cooperative Agreement No. 2008-ST-061-ED0002. Authors thank Gurit SP Technology for providing the material, as well as Dr. Stephen Nolet and TPI Composites for providing the facility for creating the composites used in this study. A special thanks to Dr. Richard Brown for providing the Scanning Electron Microscopy (SEM) facility used in obtaining the images of the cell microstructures.

\section{References}

[1] Xue Z, Hutchinson JW. Preliminary assessment of sandwich plates subject to blast loads. Int J Mech Sci 2003; 45: 687-705. 
[2] Fleck NA, Deshpande VS. The resistance of clamped sandwich beams to shock loading. J Appl Mech 2004; 71: 386-401.

[3] Dharmasena KP, Wadley HNG, Xue Z, Hutchinson JW. Mechanical response of metallic honeycomb sandwich panel structures to high-intensity dynamic loading. Int J Imp Eng 2008; 35(9): 1063-1074.

[4] Zhu F, Zhao L, Lu G, Wang Z. Deformation and failure of blast loaded metallic sandwich panels - Experimental investigations. Int J Imp Eng 2008; 35(8): 937-951.

[5] Nurick GN, Langdon GS, Chi Y, Jacob N. Behavior of sandwich panels subjected to intense air blast: part 1- Experiments. Compos Struct 2009; 91 (4): 433-441.

[6] Radford DD, Fleck NA, Deshpande VS. The response of clamped sandwich beams subjected to shock loading. Int J Imp Eng 2004; 32(6): 968-987.

[8] Tagarielli VL, Deshpande VS, Fleck NA. The high strain rate response of PVC foams and end-grain balsa wood. Compos: B 2008; 39: 83-91.

[9] Atas C, Sevim C. On the impact response of sandwich composites with cores of balsa wood and PVC foam. Compos Struct 2010; 93: 40-48.

[10] McShane GJ, Deshpande VS, Fleck NA. The underwater blast resistance of metallic sandwich beams with prismatic lattice cores. J Appl Mech 2007; 74: 352-364. [11] McShane GJ, Radford DD, Deshpande VS, Fleck NA. The response of clamped sandwich plates with lattice cores subjected to shock loading. Euro J Mech - A: Solids 2006; $25: 215-229$.

[12] Hossain MK, Liu QL, O’Toole BJ. Functionally graded foam material system for energy absorption. SAMPE $39^{\text {th }}$ ISTC- Cincinnati OH, Oct. 29 - Nov. 1, 2007.

[13] Kiernan S, Cui L, Gilchrist MD. Propagation of a stress wave through a virtual functionally graded foam. Int J Non-Linear Mech 2009; 44: 456-468.

[14] Cui L, Kiernan S, Gilchrist MD. Designing the energy absorption capacity of functionally graded foam materials. Mater Sci Eng: A 2009; 507: 215-225.

[15] Li Y, Ramesh, KT, Chin ESC. Dynamic characterization of layered and graded structures under impulsive loading. Int J Sol Struct 2001; 38(34-35): 6045-6061. 
[16] Apetre NA, Sankar BV, Ambur DR. Low-velocity impact response of sandwich beams with functionally graded core. Int J Sol Struct 2006; 43(9): 2479-2496.

[17] Wang E, Gardner N, Shukla A. The blast resistance of sandwich composites with stepwise graded cores. Int J Sol Struct 2009; 46: 3492-3502.

[18] Gardner N, Wang E, Shukla A. Performance of functionally graded sandwich beams under shock wave loading. Compos Struct 2011. DOI 10.1016/j.compstruct.2011.12.006.

[19] http://www.gurit.com/corecell-afoam.aspx. Accessed September 20, 2011.

[20] Chen W, Zhang B, Forrestal MJ. A split Hopkinson bar technique for low impedance materials. Exp Mech 1998; 39 (2): 81-85.

[21] Kumar P, LeBlanc J, Stargel D and Shukla A. Effect of plate curvature on blast response of aluminum panels. Imp Eng 2012; Accepted

[22] LeBlanc J, Shukla A, Rousseau C, Bogdanovich A. Shock loading of threedimensional woven composite materials. Compos Struct 2007; 79: 344- 355.

[23] Wright J. Shock tubes. New York: John Wiley and Sons Inc, 1961.

[24] Tiwari V, Sutton MA and McNeill SR. Assessment of High-speed Imaging Systems for 2D and 3D Deformation Measurements: Methodology Development and Validation, Exp Mech 2007; 47: 561-579.

[25] Tiwari V, Sutton MA, McNeill SR, Xu S, Deng X, Fourney WL, and Bretall D. Application of 3D Image Correlation for Full-Field Transient Plate Deformation Measurements During Blast Loading, Int J Imp Eng 2009; 36; 862-874.

[26] Gibson LJ, Ashby MF. Cellular Solids: Structures and Properties. New York: Pergamon Press, Inc., 1988.

[27] Wang E, Shukla A. Analytical and experimental evaluation of energies during shock wave loading. Int J Imp Eng 2010; 37: 1188-1196

[28] Allen H. Analysis and design of structural sandwich panels. Oxford: Pergamon Press, Inc., 1969. 
[29] Theotokoglou E. Analytical determination of the ultimate strength of sandwich beams, Appl Compos Mater 1996; 3: 354-353.

[30] Steeves CA and Fleck NA. Collapse mechanisms of sandwich beams with composite facesheets and a foam core, loaded in three-point bending. Part I: analytical models and minimum weight design, Int J Mech Sci 2004; 46; 561-583.

[31] Steeves CA and Fleck NA. Collapse mechanisms of sandwich beams with composite facesheets and a foam core, loaded in three-point bending. Part II; experimental investigation and numerical modeling, Int J Mech Sci 2004; 46; 585-608.

[32] Lim TS, Lee CS and Lee DG, Failure modes of foam core sandwich beams under static and impact loads, J Compos Mater 2002; 38 (18): 1639-1662.

[33] Chiras S, Mumm D, Evans A, Wicks N, Hutchinson J, Dharmasena H, Wadley H and Fichter S. The structural performance of near-optimized truss core panels, Int J Sol Struct 2002; 39 (15): 4093-4115.

[34] Carlsson LA and Kardomateas GA, Structural and failure mechanics of sandwich composites. New York: Springer, 2011. 


\title{
CHAPTER 6
}

\section{BLAST RESPONSE OF NANO-SCALE CORE-SHELL RUBBER (CSR) TOUGHENED SANDWICH COMPOSITES}

\author{
by \\ Nate Gardner, Sachin Gupta and Arun Shukla \\ Under preparation for submission to the International Journal of Impact Engineering \\ Corresponding Author: Arun Shukla \\ Dynamic Photo Mechanics Laboratory \\ Department of Mechanical, Industrial and Systems \\ Engineering \\ University of Rhode Island \\ 206 Wales Hall, 92 Upper College Rd \\ Kingston, RI, 02881, USA \\ Phone: +1-401-874-2283 \\ Email Address: shuklaa@egr.uri.edu
}




\begin{abstract}
The dynamic behavior of two types of sandwich composites made of E-glass VinylEster (EVE) facesheets and Corecell ${ }^{\mathrm{TM}}$ A500 foam was studied using a shock tube apparatus. The core material and thickness, as well as overall specimen dimensions were held constant, with the only difference arising in the resin system used during the infusion. The non-core-shell rubber toughened resin system (Non-CSR) consisted of a vinyl-ester resin only; while the CSR toughened resin consisted of the same vinylester resin, but with Kane Ace MX-153 nano-scale core-shell rubber particles added to the mixture. Prior to shock tube testing, the quasi-static and dynamic constitutive behavior of the facesheets (tensile/compressive) and foam (compressive)was evaluated. During the shock tube testing, high-speed photography coupled with the optical technique of Digital Image Correlation (DIC) was utilized to capture the realtime deformation process as well as mechanisms of failure. Post-mortem analysis was also carried out to evaluate the overall blast performance of these configurations. Results indicated that adding nano-scale core-shell rubber (CSR) particles to sandwich composites, aids in dispersing the initial shock wave loading, thus reducing the overall deflection, strain, and velocity and improving the overall blast resistance of the structure.
\end{abstract}

Keywords: Sandwich Structures, Polymers, Core-shell Rubber (CSR), Blast Loading, High-speed Photography

\title{
1. Introduction
}

Sandwich structures have very important applications in naval and aerospace industry. Due to their construction they have many advantages that include high 
strength/weight ratio, high stiffness/weight ratio, and energy absorption capabilities. Sandwich structures consist of two thin, stiff facesheets, usually of the same thickness, separated by a lightweight, thicker core. The facesheets carry almost all of the bending and in-plane loads, while the core helps to stabilize the facesheets and defines the flexural stiffness and out-of-plane shear and compressive behavior. When sandwich structures are subjected to high-intensity impulse loadings, such as air blasts, the core materials play a crucial role in the dynamic behavior and overall structural response. Their properties assist in dispersing the mechanical impulse that is transmitted into the structure, and thus protect anything located behind it [1-3].

Common cores are made of metallic and non-metallic honeycombs, cellular foams, balsa wood, PVC, truss and lattice structures. Extensive research exists in the literature regarding the dynamic response of sandwich structures consisting of the various core materials and geometric structures [3-9]. These studies have indicated that advanced sandwich structures can potentially have significant advantages over monolithic plates of equivalent mass in absorbing the blast energy, whether in air or underwater. Apart from the various core materials and structures, the facesheet also plays an important role in the blast mitigation properties of the structure. In fact, the facesheet is the part of the structure which is directly exposed to the blast loading.

For marine applications, fiber-composite (facesheet) materials are commonly based on epoxies and other thermosetting polymers. This is due to the fact that these thermosetting polymers are highly cross- linked resulting in materials which exhibit good elevated temperature resistance and low creep. However, their high cross-link densities cause them to be relatively brittle in nature. This limits their applications as 
structural materials, as they have a poor resistance to crack initiation and growth. To overcome this deficiency and increase toughness, a commonly used method is the addition of a second dispersed particulate phase (during infusion). This second dispersed particulate phase can either be initially soluble in the epoxy resin and which then phase separates during curing to form or it can be of pre-formed particles. For the phase-separable tougheners, both rubbers (carboxyl-terminated butadiene-acrylonitrile (CBTN) $[10,11])$ and thermoplastics [12-14] have been investigated. Pre-formed particles that have been studied include ceramic particles (glass [15, 16], alumina [17], or silica $[18,19]$ ), metal particles (aluminum [18]), polymers [20, 21] and core-shell rubber particles [22-27].

The behavior of rubber toughened and core-shell rubber toughened epoxy resin has been extensively studied in the literature $[10,11,22-27]$. The core-shell rubber particles consist of two parts, a core which is rubber for impact resistance, and a shell which is a co-polymer compatible with epoxy resin. Note for these investigations, most of these rubber particles were on the micro-scale level. Results of these investigations indicated that the addition of rubber particles to epoxy resins can aid in increasing the fracture toughness, lap shear / T-peel strength, and fatigue resistance, as well as allow for no loss of $\mathrm{Tg}$ or thermal properties (during infusion process), consistent morphology and a wide cure window. Therefore, the addition of rubber particles to current resin systems allows the once-brittle by nature resin to become toughened and subsequently more impact resistant.

Due to the improvement in mechanical properties, these rubber toughened epoxies can be used as the matrices for fiber-reinforced composite systems. However, 
the addition of these tougheners or pre-formed rubber particles, in the concentrations required to sufficiently enhance the toughness, can significantly increase the viscosity of the matrix resin. Also, conventional pre-formed particles generally have a particle diameter larger than the inter-fiber spacing, and particles are filtered out during infusion. This has led to the development of nano-scale rubber particles [27], defined as rubber particles less than $100 \mathrm{~nm}$ in diameter, since these particles will flow between the fibers during infusion [28].

Even though the behavior of rubber toughened epoxy systems has been extensively investigated in the literature, investigations regarding the behavior of glass-fiber reinforced composites and sandwich structures are limited. Therefore, the current study will investigate the influence of nano-scale core-shell rubber (CSR) particles [Kane Ace MX 153] on the behavior and performance of E-glass Vinyl-Ester (EVE) composite panels and sandwich structures. It will expand upon the authors' previous work [29-31], for which the blast performance of sandwich composites made of E-glass Vinyl-Ester (EVE) facesheets and Corecell ${ }^{\mathrm{TM}}$ A-series foam was studied. The quasi-static and dynamic constitutive behaviors of the facesheets (both with and without CSR) and foam core material were first studied using a Split Hopkinson Pressure Bar (SHPB) device. The sandwich composites were then fabricated and subjected to shock wave loading generated by a shock tube. Both sandwich composites consisted of identical materials, core thickness and overall dimensions, with the only difference arising in the resin system. The non-core-shell rubber toughened resin system (Non-CSR) consisted of a vinyl-ester resin only; while the CSR toughened resin consisted of the same vinyl-ester resin, but with Kane Ace MX- 
153 nano-scale core-shell rubber particles added to the mixture. The shock pressure profiles and real-time deformation images were carefully analyzed to reveal the failure mechanisms of these sandwich composites. Digital Image Correlation (DIC) analysis was implemented to investigate the real-time deflection, strain and velocity of the back face of the specimens. Post-mortem analysis was also carried out to evaluate the overall blast performance of these sandwich structures.

\section{Material and Specimen}

\subsection{Skin (Facesheet) and Core Materials}

The facesheet materials that were utilized in this study are E-glass Vinyl-Ester (EVE) composites. The woven roving E-glass fibers of the facesheet materials were placed in a quasi-isotropic layout $[0 / 45 / 90 /-45]_{s}$. The fibers were made of the .610 $\mathrm{kg} / \mathrm{m}^{2}$ areal density plain weave. The resin system used consisted of Ashland Derakane Momentum 8084, Ashland Derakane Momentum 411-200, and Kaneka Kane Ace MX153 (Core-shell Rubber) with the front and back skins (facesheets) consisting of identical layups and materials. Both composite panels consisted of 55\% volume fraction of glass (fiber). Fig. 1 shows a schematic of the sandwich composite with skin and core materials.

Ashland Derakane 8084 is an elastomer modified epoxy vinyl ester resin designed to offer increased adhesive strength, superior resistance to abrasion and severe mechanical stress, while giving greater toughness and elongation. Ashland Derakane 411-200 is an epoxy vinyl ester resin based on Bisphenol-A epoxy that is specifically formulated for use in vacuum infusions. Kaneka Kane Ace MX153is a 33\% concentrate core-shell rubber (CSR) toughening agent in unmodified liquid epoxy 
resin based on Bisphenol-A. The CSR of Kane Ace MX153 is based on a lower Tg rubber component which provides more toughening than standard CSR compositions. MX153 is stable and the CSR remains completely dispersed under normal handling, formulating and curing conditions. Fig. 2 shows an SEM image of nano-scale coreshell rubber particles [27].

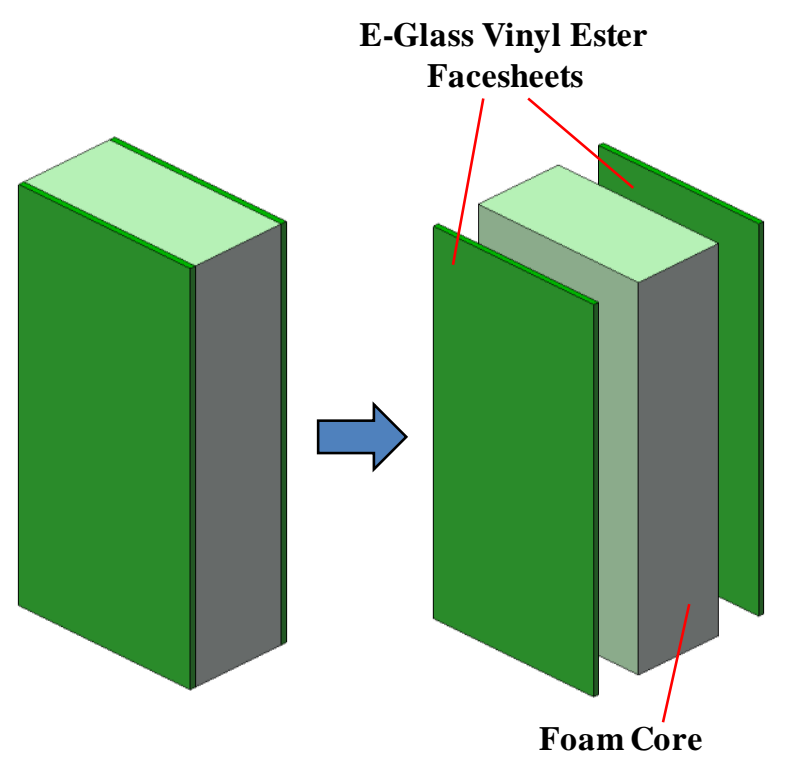

Fig. 1 Schematic of sandwich composite with skin and core

Due to the fact that resin viscosity is important during the infusion process (VARTM), the Ashland Derakane Momentum 411-200 was added to the Ashland Derakane Momentum 8084 in order to counteract the high viscosity of the Kaneka Kane Ace MX153. The dynamic viscosities of the Ashland Derakane 8084, 411-200 and Kaneka Kane Ace MX153 are $360 \mathrm{mPa}-\mathrm{s}\left(25^{\circ} \mathrm{C}\right), 210 \mathrm{mPa}-\mathrm{s}\left(25^{\circ} \mathrm{C}\right)$, and 20,000 $\mathrm{mPa}-\mathrm{s}\left(50^{\circ} \mathrm{C}\right)$, as given from the manufacturer's data [32-34]. For this investigation a mixture of $48 \%$ Ashland Derakane 8084, $48 \%$ Ashland Derakane 411-200 and $4 \%$ Kaneka Kane Ace MX 153 nano-scale core-shell rubber (CSR) particles was used to fabricate the CSR toughened composite facesheets and sandwich panels. 


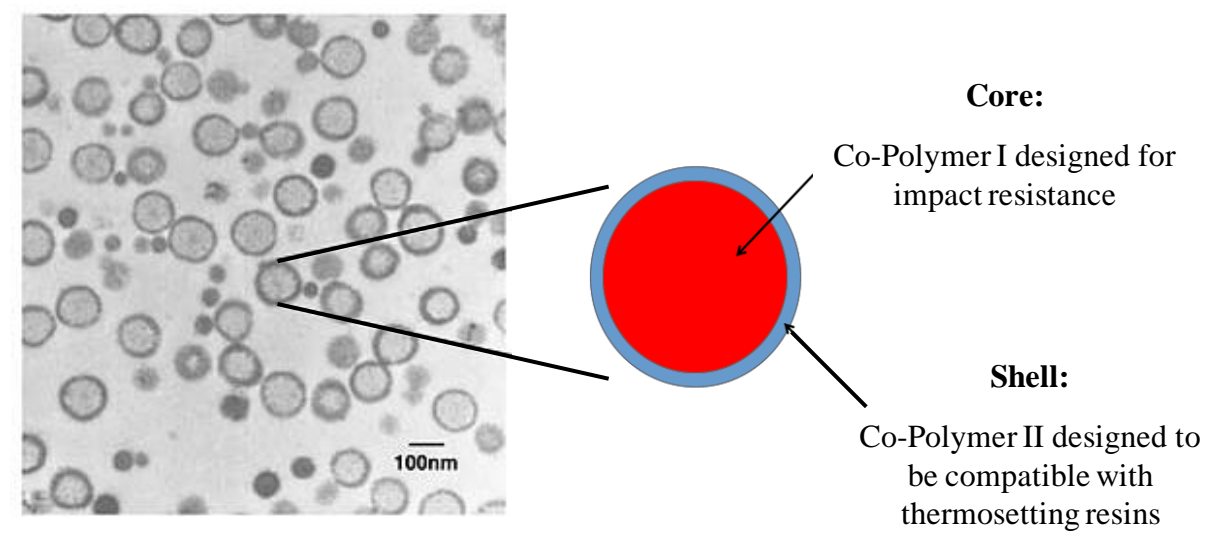

Fig. 2 SEM image of nano-scale core-shell rubber (CSR) particles [27].

The core material used in the present study is Corecell ${ }^{\mathrm{TM}}$ A500, which is a styrene acrylonitrile (SAN) foam manufactured by Gurit SP Technologies specifically for marine sandwich composite applications. Table 1 lists important material properties of the foam from the manufacturer's data [35], as well as the material properties of the facesheet [29]. The material properties of the facesheet and the core materials were determined using proper ASTM standards, D 3410 and D 1621 respectively. The SEM images of the A500 foam cell microstructure can be seen in Fig. 3.

Table 1. Quasi-static material properties of foam core [35] and EVE facesheet [29]

\begin{tabular}{ccccc}
\hline & $\begin{array}{c}\text { Nominal } \\
\text { Density, } \boldsymbol{\rho} \\
\left(\mathrm{kg} / \mathrm{m}^{3}\right)\end{array}$ & $\begin{array}{c}\text { Compressive } \\
\text { Modulus, E } \\
(\mathrm{MPa})\end{array}$ & $\begin{array}{c}\text { Compressive } \\
\text { Strength, } \boldsymbol{\sigma}_{\mathbf{y}} \\
(\mathrm{MPa})\end{array}$ & $\begin{array}{c}\text { Acoustic Wave } \\
\text { Impedance } \\
\left(\mathrm{kg} / \mathrm{m}^{2} \mathrm{~s}\right)\end{array}$ \\
\hline $\mathbf{A 5 0 0}$ & 92 & 64 & 0.9 & $7.7 \times 10^{4}$ \\
$\begin{array}{c}\text { E-glass } \\
\text { Vinyl-Ester } \\
\text { Composite }\end{array}$ & 1800 & $\begin{array}{c}13600 \\
{[\text { longitudinal }]} \\
3900\end{array}$ & 220 & $\begin{array}{c}4.9 \times 10^{6} \\
{\left[\begin{array}{c}\text { longitudinal] } \\
2.6 \times 10^{6} \\
{[\text { transverse] }}\end{array}\right.}\end{array}$ \\
\hline
\end{tabular}




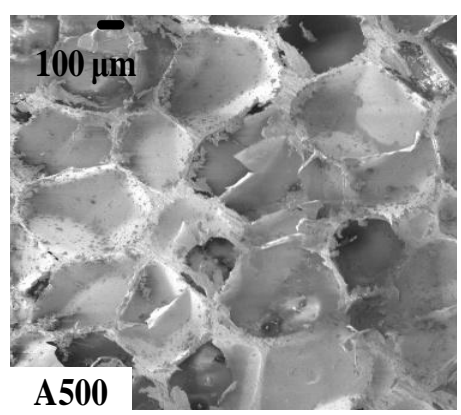

Fig. 3 Cell microstructure of A500 foam core layer [35]

\subsection{Core-shell Rubber (CSR) Toughened Sandwich Composites}

Vacuum Assisted Resin Transfer Molding (VARTM) process was utilized to fabricate the sandwich specimens. During the VARTM process, the sandwich specimens were infused under the same conditions, i.e. temperature, humidity, and vacuum pressure $(760 \mathrm{mmHg}(1 \mathrm{~atm}))$, with the same volume of resin. The overall dimensions for the samples were approximately $102 \mathrm{~mm}$ wide, $254 \mathrm{~mm}$ long and 48 $\mathrm{mm}$ thick. The foam core itself was approximately $38 \mathrm{~mm}$ thick, while the skin thickness was approximately $5 \mathrm{~mm}$.

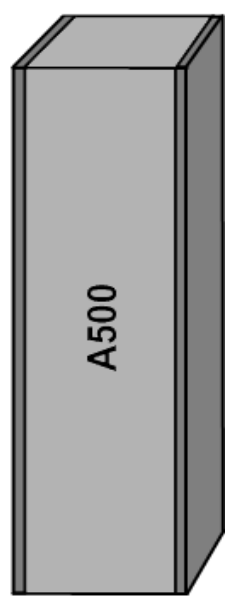

(a) Specimen Schematic

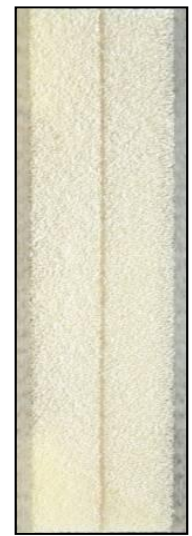

(b) A500 foam core
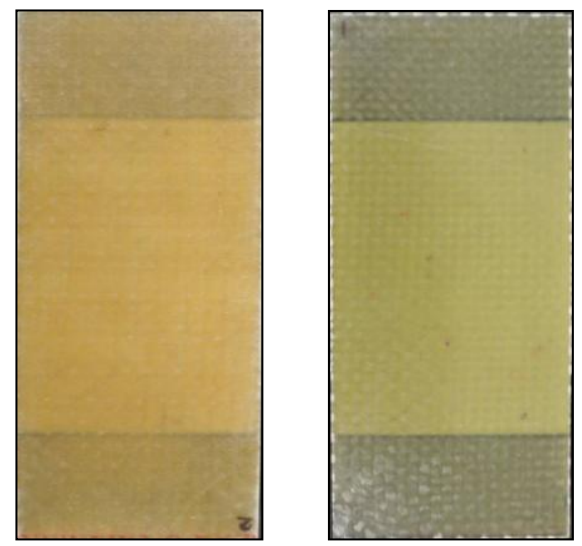

(c) Different facesheet material systems; (L) Non-CSR, (R) CSR

Fig.4 Specimen design and facesheet configurations 
For the sandwich composites utilized in this study, two different resin systems were used; one configuration utilized a simple vinyl-ester resin system, while the other configuration utilized the same vinyl-ester resin system but with nano-scale core-shell rubber (CSR) toughening. The sandwich composite panels can be seen in Fig. 4. Both configurations consisted of one core layer of foam, A500. Since the core material and thickness, as well as overall specimen dimensions were identical, with the only difference arising in the resin system used during the infusion, the areal density of the two configurations was within $3 \%$, i.e. the areal density of the Non-CSR toughened sandwich composite was $19 \mathrm{~kg} / \mathrm{m}^{2}$, and the CSR toughened sandwich composite was $18.5 \mathrm{~kg} / \mathrm{m}^{2}$

\section{Experimental Set-up and Procedure}

\subsection{Quasi-Static Loading}

The quasi-static loading was implemented by a screw- driven testing machine (Instron 5582), as shown in Fig. 5. For the quasi-static tensile and compressive tests on the facesheet materials, the proper ASTM standards for polymer matrix composite materials were used, ASTM D3039/D3039M - 08 (tensile) and ASTM D3410/D3410M - 03 (compressive). The experimental set-up consisted of the Instron 5582 testing machine, an extensometer ( $25.4 \mathrm{~mm}$ gage section) and a data acquisition system, along with a Wheatstone bridge high-strain indicator and oscilloscope (Tektronix TDS 3014). Note for the tensile experiments, two strain gages (C2A-13250LW-350, Vishay Micro-measurements) were placed on the panels to ensure no shear loading is being applied. These gages were placed longitudinally and centered approximately in the middle of the specimen (length), centered $5 \mathrm{~mm}$ from the right 
and left side. For the compressive tests both gages were placed longitudinally, one gage on each side of the panel (front and back). Specimens were loaded with a constant crosshead rate of $2 \mathrm{~mm} / \mathrm{min}$ and $1.5 \mathrm{~mm} / \mathrm{min}$ for the tensile and compressive tests respectively. For the tensile loading, an extensometer was utilized to obtain the exact deformation of the specimen, while under compressive loading the machine compliance was subtracted from the total deformation to obtain the exact deformation of the specimen. Specimen dimensions were approximately $25.4 \mathrm{~mm}$ wide, $254 \mathrm{~mm}$ long and $5 \mathrm{~mm}$ thick (tensile) and $25.4 \mathrm{~mm}$ wide, $152 \mathrm{~mm}$ long and $5 \mathrm{~mm}$ thick (compression). For the transverse compressive loading of the facesheet material, circular specimens with a diameter of approximately $12.7 \mathrm{~mm}$, and thickness of $5 \mathrm{~mm}$ were used. Specimens were loaded with a constant crosshead rate of $0.25 \mathrm{~mm} / \mathrm{min}$.

For the quasi-static compressive tests of the core material, the proper ASTM standard for rigid cellular plastics (foam) was used, ASTM D1621 - 04a. Specimens were loaded with a constant crosshead rate of $2.5 \mathrm{~mm} / \mathrm{min}$. Specimen dimensions were approximately $25.4 \mathrm{~mm}$ wide, $25.4 \mathrm{~mm}$ long, and approximately $12.7 \mathrm{~mm}$ thick. 


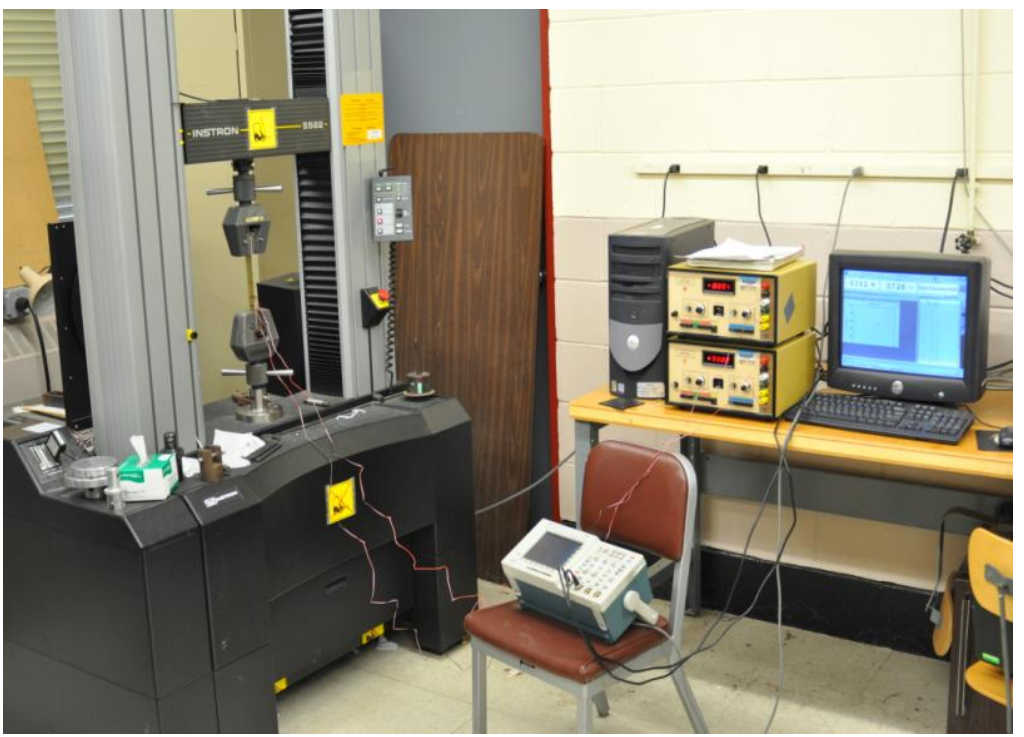

(a) Instron 5582 tensile experimental set-up (facesheets)

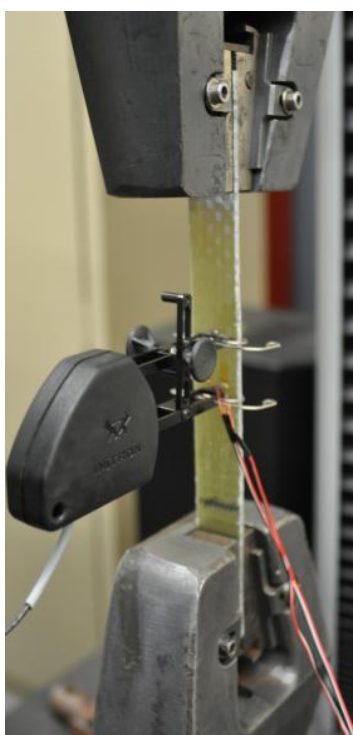

(b) Close up of specimen, along with extensometer and strain gages

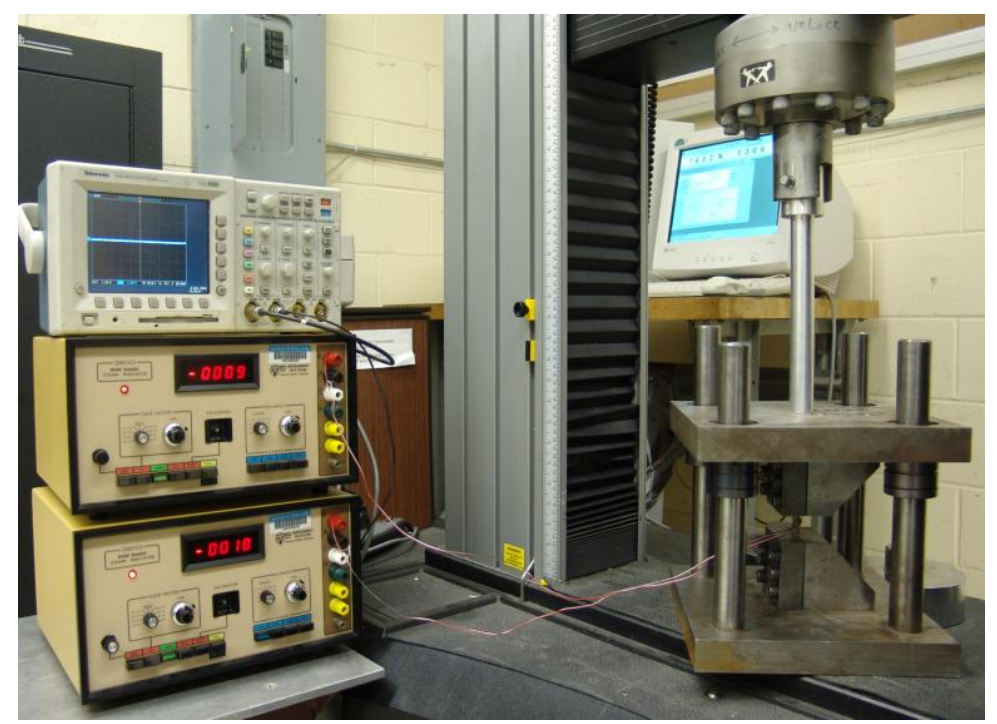

(c) Instron 5582compressive experimental set-up

Fig. 5 Quasi-static experimental set-up

\subsection{Drop-weight Impact Tower}

The dynamic loading was implemented by a drop-weight impact tower apparatus (Instron 9210), as shown in Fig. 6. To investigate the dynamic impact behavior of the facesheets, the proper ASTM standard for polymer matrix composite materials was utilized (ASTM D 7136/D 7136M - 07). The Dynatup 9210 used is outfitted with a 
medium weight crosshead and is capable of producing 4.6-300 Joules of energy. The maximum impact velocity that can be achieved is $5 \mathrm{~m} / \mathrm{s}$. The drop tower is equipped with a data acquisition system. The data acquisition system includes a velocity detector, a piezoelectric tup for measuring loads, a signal conditioning amplifier, and a computer with an A/D board. The velocity detector is capable of measuring impact velocity as well as rebound velocity.

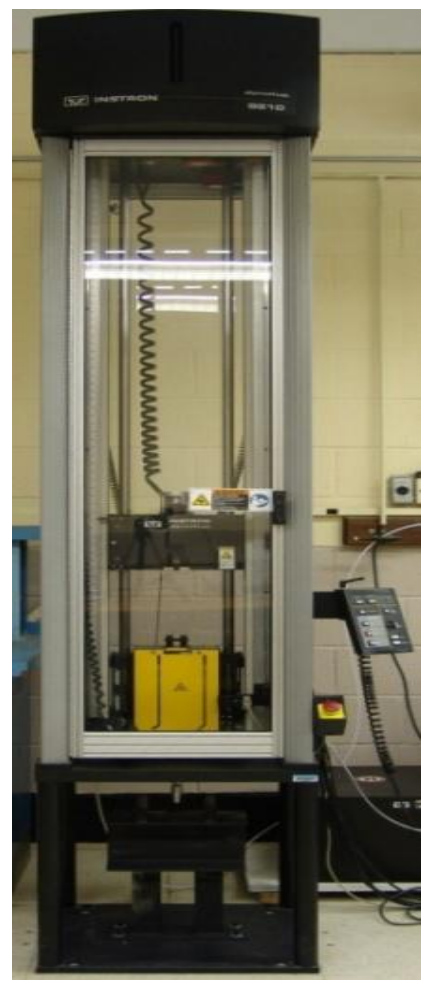

(a) Instron Dynatup dropweight tower

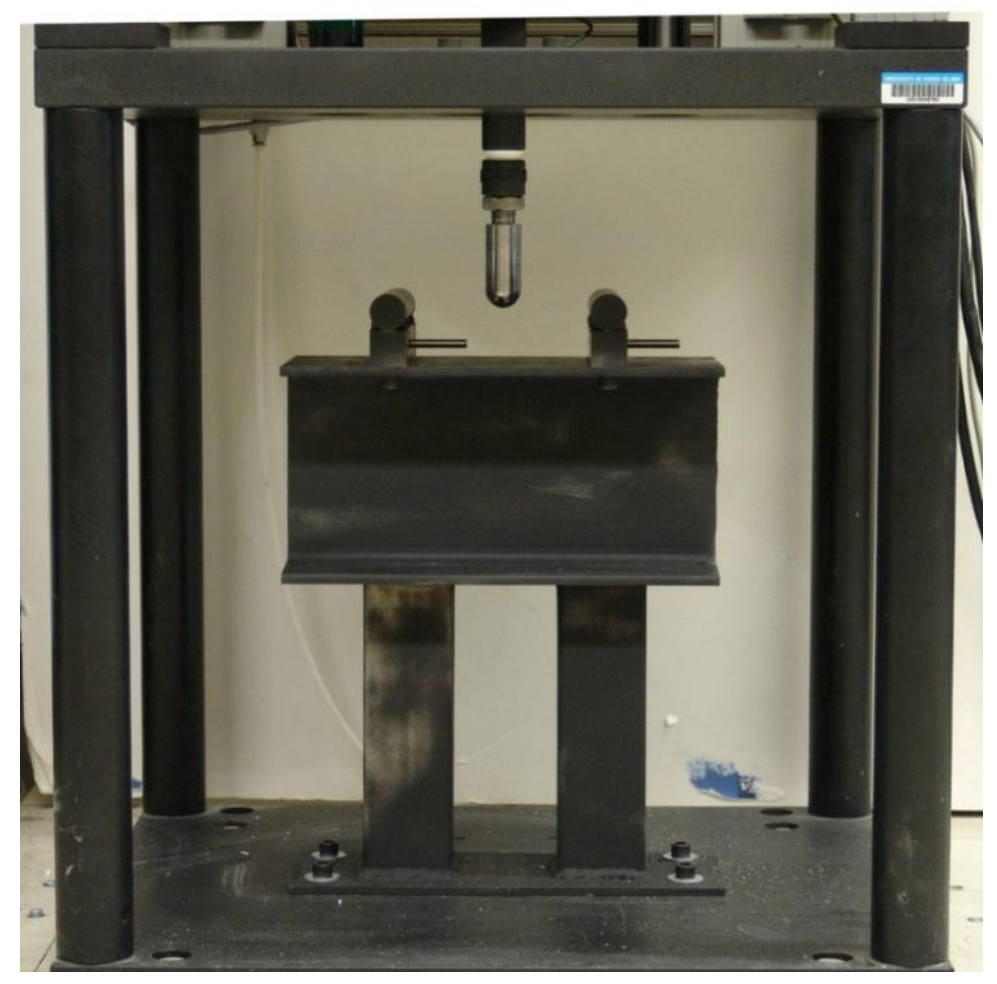

(b) Simple support fixture

Fig. 6 Drop-weight impact experimental set-up

Normally specimens are held within the drop tower enclosure during experiments. To allow the use of an environmental chamber or testing of specimens too large to fit in the enclosure, the 9210 can be modified. Specimens that do not fit in the enclosure can be fixed within the support base, outside of the enclosure. A simple support fixture 
was built that resides in the support base (see Figure 6b). Note the span width between supports is approximately $152 \mathrm{~mm}$, which is identical to the simple support span in the shock loading experiments. The positioning of the specimen outside of the enclosure also allowed the specimen to be oriented in such a way that a high-speed photography system could be employed during testing. The use of the high-speed photography system was to ensure proper results, i.e. duration of event (prior to impacting support fixture), no slipping of the specimen, and proper loading (center).

To perform an experiment several steps must be taken. The crosshead mass and drop height must be determined. Given that highest energy output was to be used the cross head was loaded with the maximum weight. All weights are stamped with their mass. The additional mass of the crosshead, reaction plate, reaction plate bolts, tup, tup bolts, and striker were taken into account. The mass of the crosshead, reaction plate and bolts are labeled from the manufacturer. The tup, tup bolt and striker were weighed to determine their mass. Table 2 shows the mass of all components contributing to the impact.

Table 2. Mass of drop-weight components contributing to impact

\begin{tabular}{|c|c|c|c|c|c|c|}
\hline Component & $\begin{array}{c}\text { Reaction } \\
\text { plates } \\
\text { and bolts }\end{array}$ & $\begin{array}{c}\mathbf{4 4} \mathbf{~ K N} \\
\mathbf{1 0 , 0 0 0} \text { lb) } \\
\text { tup and } \\
\text { bolt }\end{array}$ & $\begin{array}{c}\mathbf{2 5 . 4} \text { mm (1in) } \\
\text { hemispherical } \\
\text { striker }\end{array}$ & $\begin{array}{c}\text { Cross- } \\
\text { head }\end{array}$ & Weights & $\begin{array}{c}\text { Total } \\
\text { Mass }\end{array}$ \\
\hline Mass (kg) & 1.39 & 0.85 & 0.31 & 4.79 & 11.16 & 19.5 \\
\hline
\end{tabular}

Both facesheet materials were subjected to approximately $150 \mathrm{~J}$ of a low velocity impact with a $25.4 \mathrm{~mm}$ (1in) diameter hemispherical striker installed on the tup. Given the impact energy and known mass of the drop-weight, the drop height, $h$, was determined by 


$$
h=\frac{E}{m g}
$$

where $\mathrm{E}$ is the desired energy, $\mathrm{m}$ is the mass of the drop-weight, and $\mathrm{g}$ is the acceleration due to gravity.

After the drop height was determined, the drop tower velocity was tested. The specimen was placed in the fixture and the cross head was lowered until it came into contact with the specimen. The velocity sensor must be adjusted so that the velocity flag attached to the crosshead is in line with the bottom of the sensor. With the sensor adjusted, the number indicated on the scale was taken as a datum point and the calculated drop height was set from the datum. The crosshead was raised to the appropriate height and the specimen removed. A velocity test was then completed to ensure that the proper velocity was reached. Impact velocities were checked against a calculated velocity determined by

$$
v=\sqrt{2 g h}
$$

Before experiments were performed, the data acquisition system was configured. Each tub has a calibration factor that must be input into the software. The system was configured using the correct calibration factor for the $44 \mathrm{KN}(10,000 \mathrm{lb})$ tup. After the calibration factor was entered, the sampling rate was properly chosen. The sampling rate will determine if the entire event is captured. The data acquisition system will record 8192 data points regardless of the sampling rate, therefore it is important to know the duration of the impact event. For the given study, the event duration was approximately $12 \mathrm{~ms}$. A sampling rate of $410 \mathrm{kHz}$ was chosen as this corresponds to 20 ms allowing for a proper margin of safety. Figure 7 shows a specimen placed in the simple supports with the hemispherical impactor in contact with the specimen. 


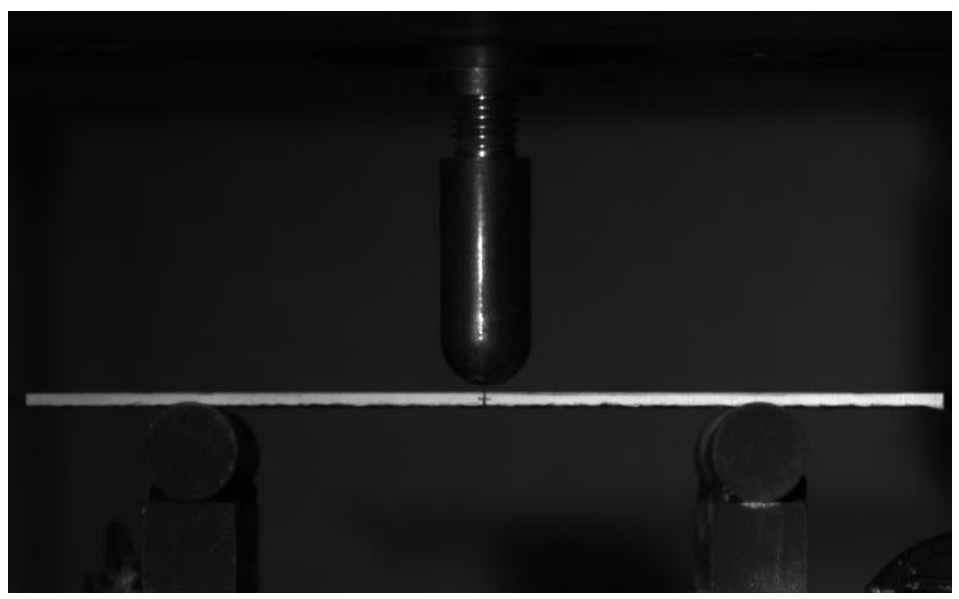

Fig. 7 Specimen placed in simply-supported conditions for dropweight impact event

\subsection{Split Hopkinson Pressure Bar (SHPB)}

A Split Hopkinson Pressure Bar (SHPB) is the most common device for measuring dynamic constitutive properties of materials. For the current investigation two different SHPB systems were utilized; solid incident-solid transmission bar for high-impedance materials and a solid incident-hollow transmission bar (Modified SHPB) for low-impedance materials. Due to the low-impedance of Corecell ${ }^{\mathrm{TM}}$ foam material, dynamic experiments for the core materials were performed with a modified SHPB device with a hollow transmission bar to increase the transmitted signal intensity. A sketch of the modified SHPB device and typical pulse profiles are given in Fig. 8. It has a $304.8 \mathrm{~mm}$-long striker, $1600 \mathrm{~mm}$-long incident bar and $1447 \mathrm{~mm}$-long transmission bar. All of the bars are made of a 6061 aluminum alloy. The nominal outer diameters of the solid incident bar and hollow transmission bar are $19.05 \mathrm{~mm}$. The hollow transmission bar has a $16.51 \mathrm{~mm}$ inner diameter. At the head and at the end of the hollow transmission bar, end caps made of the same material as the bar were press fitted into the hollow tube. By applying pulse shapers, the effect of the end 
caps on the stress waves can be minimized. The details of the analysis and derivation of equations for analysis of experimental data can be found in ref [36].

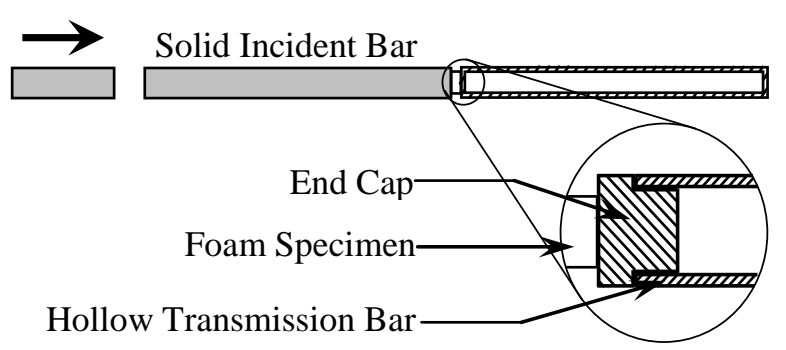

(a) Modified SHPB device

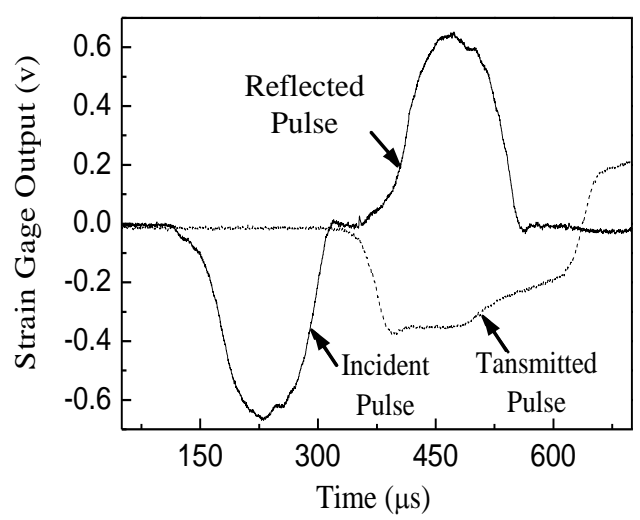

(b) Typical pulse profiles

Fig. 8 Sketch of modified SHPB device with hollow transmission bar and typical pulse profiles

Due to the high-impedance of the EVE composite facesheets, dynamic experiments were performed with a normal SHPB device (solid incident-solid transmission). . It has a $203.2 \mathrm{~mm}$-long striker, $1600 \mathrm{~mm}$-long incident bar and 1447 mm-long transmission bar. All of the bars are made of Maraging steel. The nominal outer diameters of the solid incident bar and solid transmission bar are $12.7 \mathrm{~mm}$.

\subsection{Shock Tube}

The shock tube apparatus used to obtain the controlled dynamic loading is shown in Fig. 9(a). Shock tubes offer the advantages of plane wave fronts, wave parameters that are easily controllable and repeatable, and uniform loading over shock tube muzzle diameter [37]. A complete description of the shock tube and its calibration can be found in ref. [38]. In principle, the shock tube consists of a long rigid cylinder, divided into a high-pressure driver section and a low pressure driven section, which are separated by a diaphragm. By pressurizing the high-pressure driver section, a pressure difference across the diaphragm is created. When this pressure differential 
reaches a critical value, the diaphragm ruptures. The subsequent rapid release of gas creates a shock wave, which travels down the shock tube to impart shock loading on the specimen at the muzzle end.

When the shock wave impacts the specimen located at the end of the muzzle, the wave is reflected at a higher pressure than that of the incident shock pressure. The theoretical details on the equations for shock tubes have been previously established in the literature [39]. There are four basic theoretical assumptions which are used to describe the gas flow in shock tube:

1. The gas flow is one-dimensional.

2. The gas is ideal and has constant specific heats.

3. Heat transfer and viscosity effects are neglected.

4. Diaphragm rupture is instantaneous and does not disturb the subsequent gas flow.

Using conservation of energy, mass, and momentum as described by Wright [39], the following relationships for pressure, temperature and density across a shock front can be derived:

$$
\begin{gathered}
\frac{P_{2}}{P_{1}}=\frac{2 \gamma M_{1}^{2}-(\gamma-1)}{\gamma+1} \\
\frac{T_{2}}{T_{1}}=\frac{\left\{2 \gamma M_{1}^{2}-(\gamma-1)\right\}\left\{(\gamma-1) M_{1}^{2}+2\right\}}{(\gamma+1)^{2} M_{1}^{2}} \\
\frac{\rho_{2}}{\rho_{1}}=\frac{M_{1}^{2}(\gamma+1)}{(\gamma-1) M_{1}^{2}+2}
\end{gathered}
$$

where, $P_{1}, T_{1}$ and $\rho_{1}$, are pressure, temperature and density ahead of the shock front and, $\mathrm{P}_{2}, \mathrm{~T}_{2}$ and $\rho_{2}$, are the pressure, temperature and density behind the shock front, $\gamma$ is the adiabatic gas constant, and $M_{1}$ is the mach number of the shock wave relative to 
the driven gas. The pressure imparted on the specimen can be controlled by varying the above parameters in equations 1,2, and 3. Different gases, such as nitrogen, and helium, were used in the shock tube and it was found that helium is the most suitable gas to replicate blast loading conditions and also offered the added advantage of repeatability.

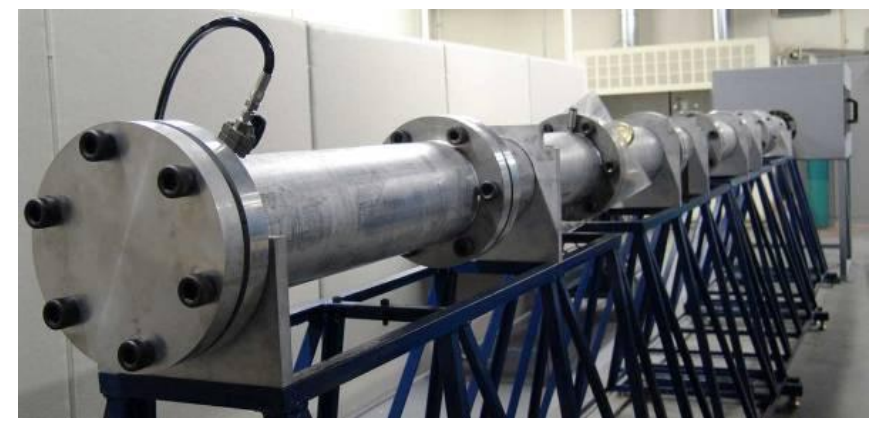

(a) Shock tube facility at URI

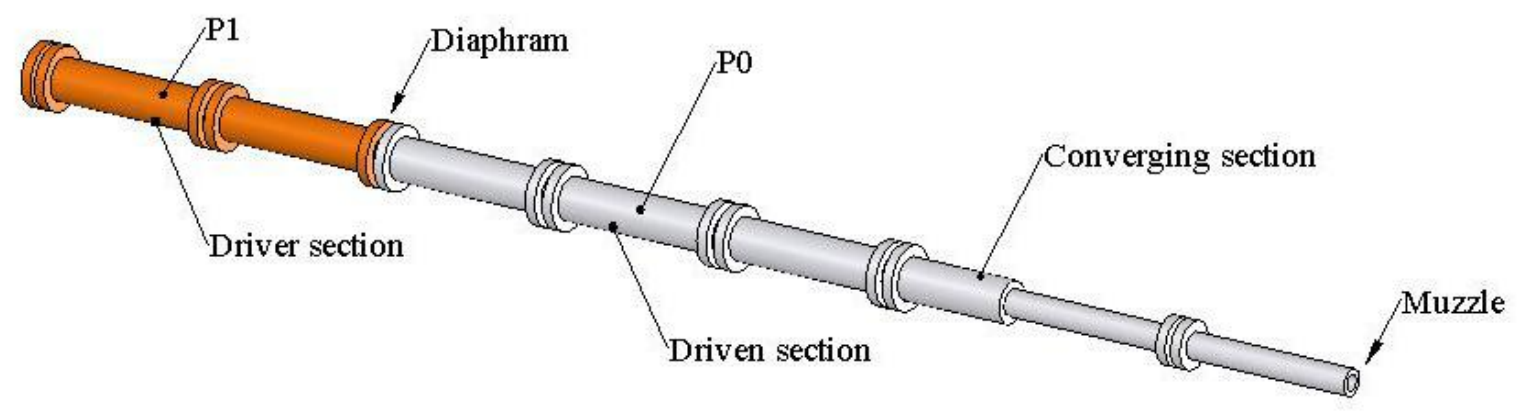

(b) Schematic of shock tube

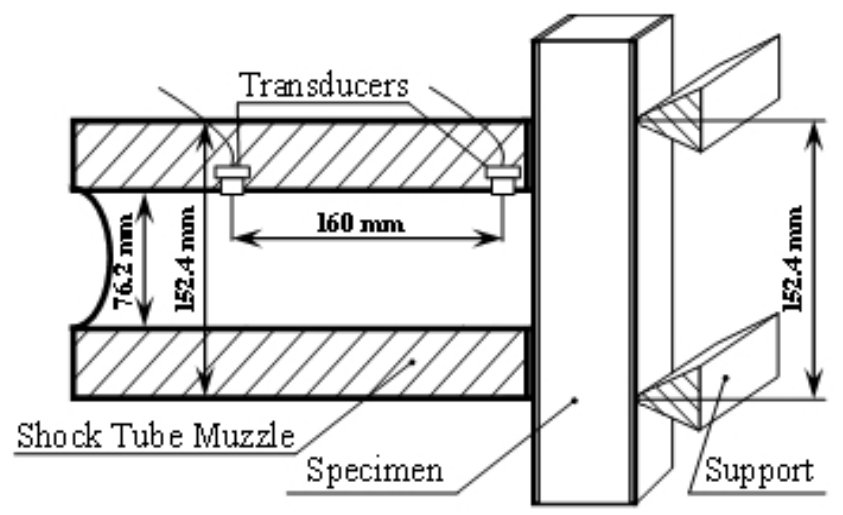

(c) Detailed dimensions of the muzzle

Fig. 9 Shock tube apparatus 
The shock tube apparatus utilized in the present study has an overall length of 8 $\mathrm{m}$, consisting of a driver, driven, converging and muzzle section. The diameter of the driver and driven section is $0.15 \mathrm{~m}$. The final muzzle diameter is $0.07 \mathrm{~m}$. Fig. $9 \mathrm{~b}$ shows detailed dimensions and locations of the muzzle, specimen, supports and the pressure sensors (PCB102A). The sensors are mounted at the end of the muzzle section to measure the incident pressure and the reflected pressure during the experiment. The final muzzle diameter is $0.076 \mathrm{~m}$. The distance between the two sensors is $0.160 \mathrm{~m}$ and the distance between the second sensor and the end of the muzzle is $\sim 0.020 \mathrm{~m}$. The specimen was placed in the supports and positioned close to the end of the muzzle. These support fixtures ensure simply supported boundary conditions with a $0.152 \mathrm{~m}$ span.

\subsection{High-speed Photography Systems}

Two high-speed photography systems were used in the present study, as shown in Fig. 10. A high-speed 3-D Digital Image Correlation (DIC) system, consisting of two high-speed digital cameras [Photron SA1], was placed facing the back side of the specimen to obtain the real-time full-field in-plane strain, along with out-of-plane deflection and velocity of the back facesheet. A randomized speckle pattern was placed directly on the back facesheet of the sandwich composite to ensure good contrast of the images. Another high-speed digital camera, [Photron SA1], was placed perpendicular to the side surface of the specimen to capture the side-view deformation images and mechanisms of failure. A framing rate of 20,000 fps was utilized which gives an interframe time of approximately $50 \mu \mathrm{s}$. 
The Digital Image Correlation (DIC) technique is utilized to capture the real-time full-field response of the back face of the sandwich composite panels. It is a nonintrusive, optical technique which allows for the capturing of the real-time dynamic response of sandwich composites through the use of high-speed photography and specialized software (PFV). In order to capture the three-dimensional response of the specimens, two cameras must be used in stereo configuration. This means the cameras must be calibrated and have synchronized image recording throughout the entire blast event. The calibration of the cameras is performed by placing a grid containing a known pattern of points (dots) in the exact location as to where the specimen will be placed during the experiment. This grid is then translated and rotated both in and outof-plane, while manually recording a series of images. Due to the fact that the grid pattern has predetermined spacing, the coordinates of the center of each point (dot) is extracted from each image. Since the coordinate location of each dot is extracted uniquely for each camera, this allows for a correspondence of the coordinate system between cameras (Tiwari et al. [40]). DIC post-processing is performed utilizing the image pairs that are recorded during the blast loading event. Using VIC-3D software package, as distributed by Correlated Solutions, common pixel subsets of the randomized speckle pattern are matched between the deformed images and the undeformed image. By matching the pixel subsets of the random speckle pattern, the three-dimensional location of distinct points on the face of the panel throughout time is obtained. This technique has been applied as a full-field measurement technique in many applications, including shock loading (Tiwari, et al. [41]). 


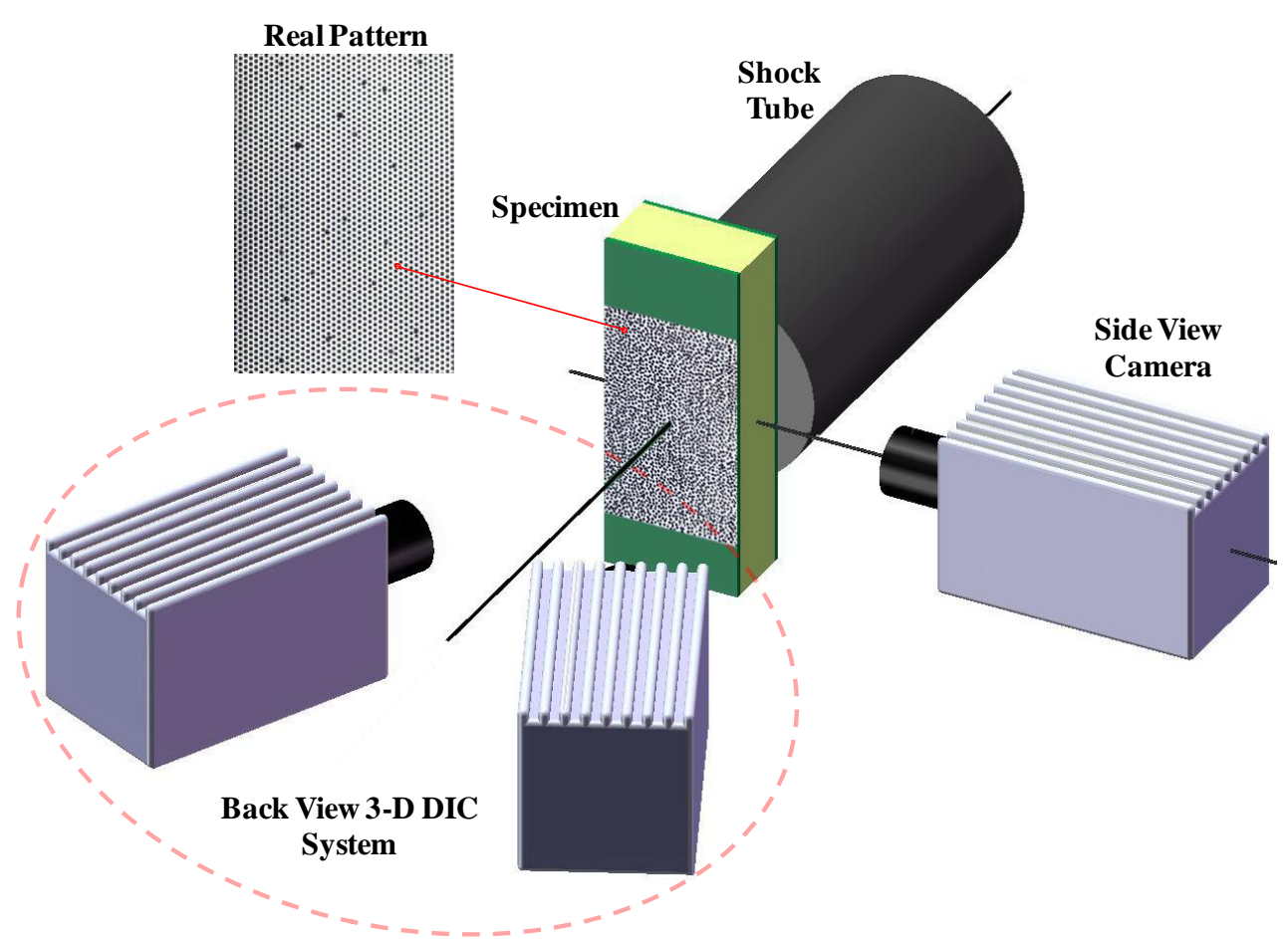

Fig. 10 High-speed photography set-up (Back-view DIC and side-view)

\subsection{Experimental Procedure and Parameters}

An initial series of experiments was conducted for both configurations and three samples were tested for each. This was followed by a second set of experiments, in which two specimens were tested for each configuration. Two different loading conditions were applied. For the first set of experiments, a simply stacked diaphragm of 5 plies of $0.254 \mathrm{~mm}$ mylar sheets with a total thickness of $1.270 \mathrm{~mm}$ was utilized to generate an impulse loading on the specimen with an incident peak pressure of approximately 1.0 $\mathrm{MPa}$, a reflected peak pressure of approximately 5.0 $\mathrm{MPa}$ and a wave speed of approximately $1000 \mathrm{~m} / \mathrm{s}$. A typical pressure profile obtained from the transducer closest to the specimen ( $\sim 0.02 \mathrm{~m}$ away) can be seen in Fig. 11. It should be noted that both pressure transducers were utilized to obtain the shock wave history, i.e. incident / reflected pressure and incident / reflected velocity. However, only the 
pressure transducer closest to the specimen was used to obtain the pressure applied on the specimen.

For the second set of experiments, a simply stacked diaphragm of 1 ply of 0.254 mm mylar sheets with a total thickness of $0.254 \mathrm{~mm}$ was utilized to generate an impulse loading on the facesheets only, with an incident peak pressure of approximately $0.3 \mathrm{MPa}$, a reflected peak pressure of approximately $1 \mathrm{MPa}$, and a wave speed of approximately $650 \mathrm{~m} / \mathrm{s}$. Due to the authors previous work [29-31], the experiments corresponding to the loading conditions with an incident peak pressure of 1.0 MPa will be presented and discussed in detail, while the facesheet experiments corresponding to the lower loading conditions (incident peak pressure 0.3 $\mathrm{MPa}$ ) will be utilized to better evaluate the performance and failure mechanisms of the sandwich structures.

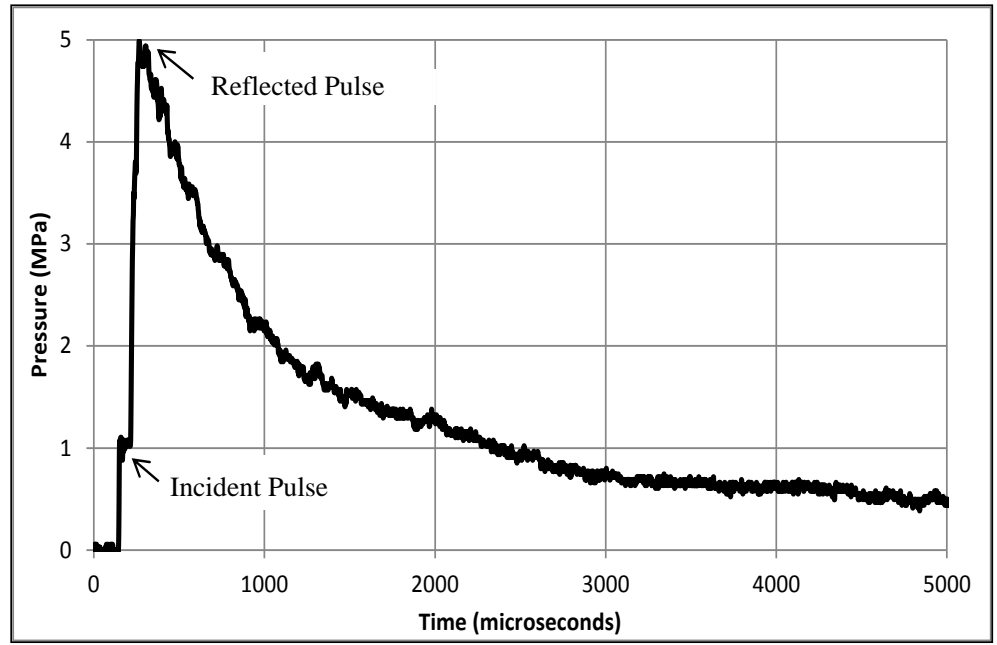

Fig. 11 Incident and reflected pressure profile 


\subsection{Residual Compressive Strength Measurements}

Following the blast loading experiments, residual strength measurements of the facesheet materials were taken. For this investigation, two specimens were tested for each configuration. The compressive strength was chosen as the residual strength parameter for this investigation. It is generally the most severely affected by impact regarding all of the mechanical properties given that the major mode of impact damage is most often delamination. Delamination is extremely detrimental to the residual compressive strength. The equipment used to measure the residual strength consisted of an Instron 5585 screw-driven testing machine, and a special compression fixture, Fig. 12, designed to be similar to that described in [42] and ASTM standard D 7137/ D7137M - 07.

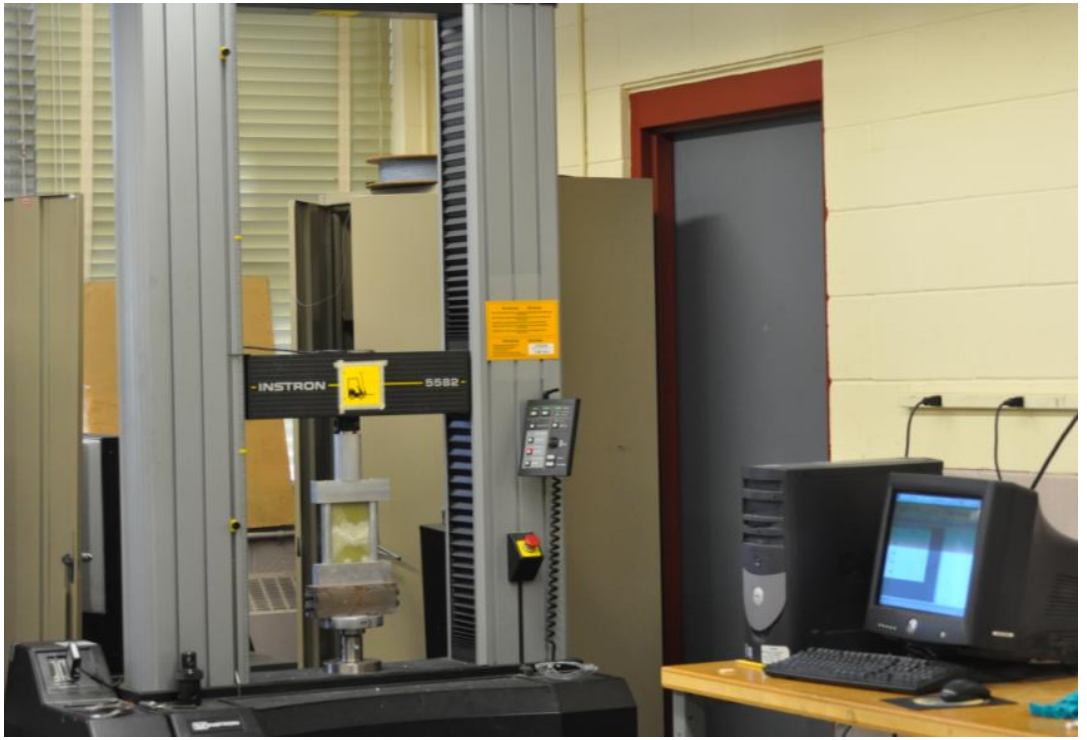

(a) Instron 5582 compressive residual strength experimental set-up (pre-damaged facesheets)

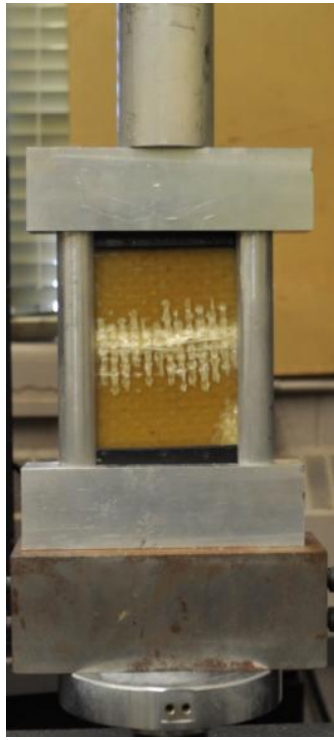

(b) Close up of predamaged specimen in compressive fixture

Fig. 12 Compressive residual strength experimental set-up

The compression fixture consists of two rigid blocks connected by two $25.4 \mathrm{~mm}$ diameter guide rods. During the experiment, the compressive load was applied to the 
sample through the top block. The top block was free to slide up or down on the guide rods. The guide rods were used to ensure proper alignment of the load. The sample was loaded in two $5.0 \mathrm{~mm}$ deep slots, in between the two blocks. The fit in the slots was tight to provide clamped edge conditions on the loaded edge of the sample. The vertical edges of the sample were supported laterally by anti-buckling guides.

The post-damaged samples were trimmed down to $101.6 \mathrm{~mm}$ wide by $152.4 \mathrm{~mm}$ long. The fixture was placed into the loading frame of the Instron 5582 testing machine and the panels were loaded with a constant crosshead rate of $1.25 \mathrm{~mm} / \mathrm{min}$ until failure. Data from this test consisted of the maximum load the plate could withstand before failure.

\section{Experimental Results and Discussion}

\subsection{Constitutive Behavior of Facesheet and Core Material}

\subsubsection{Quasi-Static Behavior}

The quasi-static behavior of the facesheet and core materials were first investigated using an Instron 5582 screw-driven testing machine. The compressive properties of the A500 foam, as well as the tensile and compressive properties of the two composite facesheet panels (Non-CSR and CSR toughened) was studied to better understand the individual behavior of all of the constituents used in the sandwich composite structure. Understanding the individual properties of each material will allow for a better understanding of the entire material system, i.e. sandwich structure. Due to the fact that during a shock wave loading event the facesheet materials exhibit very little compression(transverse and longitudinally), only the tensile behavior of the facesheet material will be presented here, as shown in Fig. 13a. 


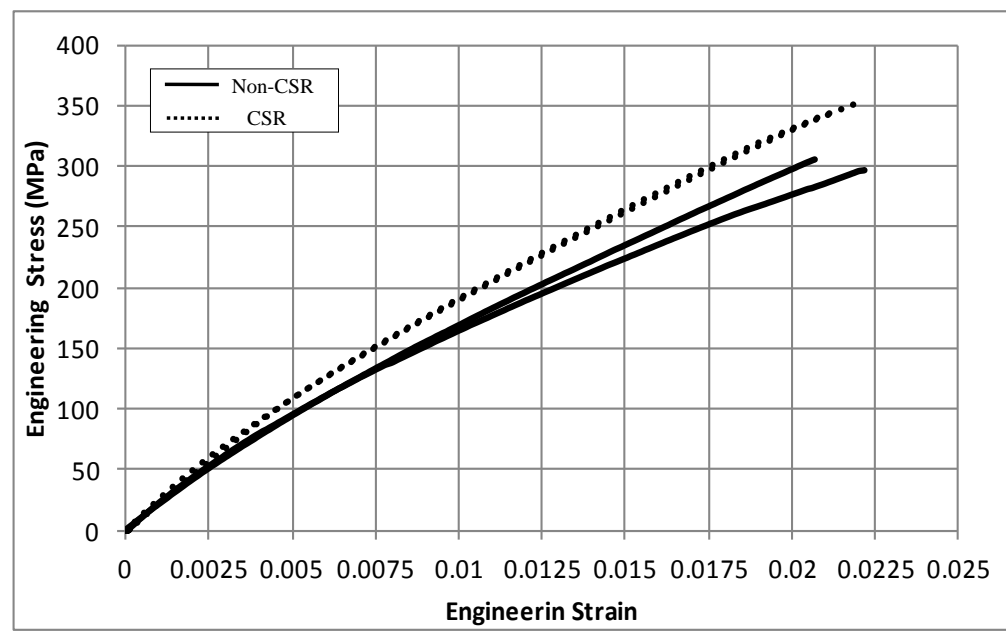

(a) Quasi-static behavior of the different composite facesheets (Non-CSR and CSR)

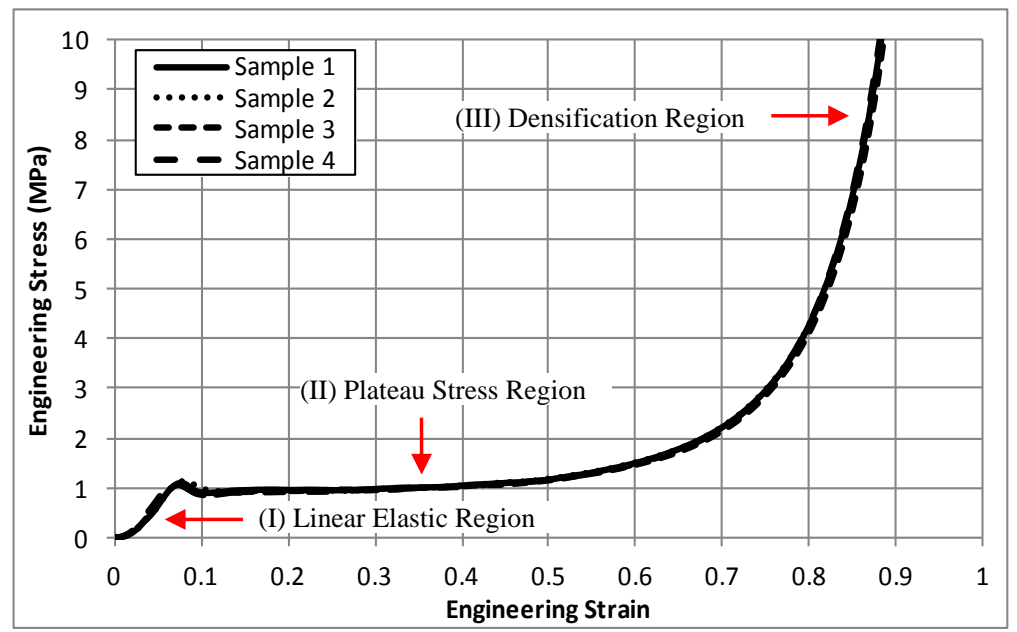

(b) Quasi-static behavior of the Corecell ${ }^{\mathrm{TM}}$ A500 foam

Fig. 13 Quasi-static behavior of composite facesheets (tensile) and A500 foam core (compressive)

For the tensile behavior of the facesheets, it can be observed that the CSR toughened composite exhibits a stiffer behavior in comparison to the Non-CSR composite panel. The average tensile Young's Modulus, E, and ultimate tensile strength, $\mathrm{U}_{\mathrm{T}}$, was approximately $14.5 \mathrm{GPa}$ and $300 \mathrm{MPa}$ for the Non-CSR and $16 \mathrm{GPa}$ and $350 \mathrm{MPa}$ for the CSR toughened facesheet respectively. Therefore, it can be 
concluded that the overall tensile strength and stiffness increases approximately $10 \%$ for the CSR toughened facesheet over the Non-CSR facesheet.

For the quasi-static compressive behavior of the A500 foam core material, as shown in Fig. 13b, the stress-strain curves show three deformation regions; (I) the linear elastic region, (II) the plateau stress (plastic yielding) region and (III) the densification region [43]. It can be observed that the plateau stress of the A500 foam is approximately $0.88 \mathrm{MPa}$.

\subsubsection{Drop-weight Impact}

Both types of composite panels were subjected to low velocity high mass (LVHM) impact events using an Instron Dynatup 9210 drop-weight tower apparatus. The specimens were simply-supported, across a $152 \mathrm{~mm}$ span (identical support conditions and span length as in shock tube experiments). The $19.4 \mathrm{~kg}$ mass was released from a drop height of $75 \mathrm{~cm}$, achieving a maximum impact velocity of 3.8 $\mathrm{m} / \mathrm{s}$ and a total impact energy of $\sim 150 \mathrm{~J}$. Load data and velocity data were recorded by a data acquisition system, and the total energy absorbed during the impact event was obtained through the data collected. For each composite panel system, at least three samples were tested. Due to the repeatability, Fig. 14 shows the total energy absorbed during the $150 \mathrm{~J}$ impact event (typical response).

It can be observed from the figure that by $\mathrm{t}=12 \mathrm{~ms}$, the Non-CSR toughened facesheet has absorbed approximately $25 \mathrm{~J}$, while the CSR toughened facesheet has absorbed $27 \mathrm{~J}$. Therefore, it can be concluded that the addition nano-scale CSR particles allows for more energy to be absorbed during impact, approximately $7 \%$ more in comparison to the Non-CSR toughened facesheet. 


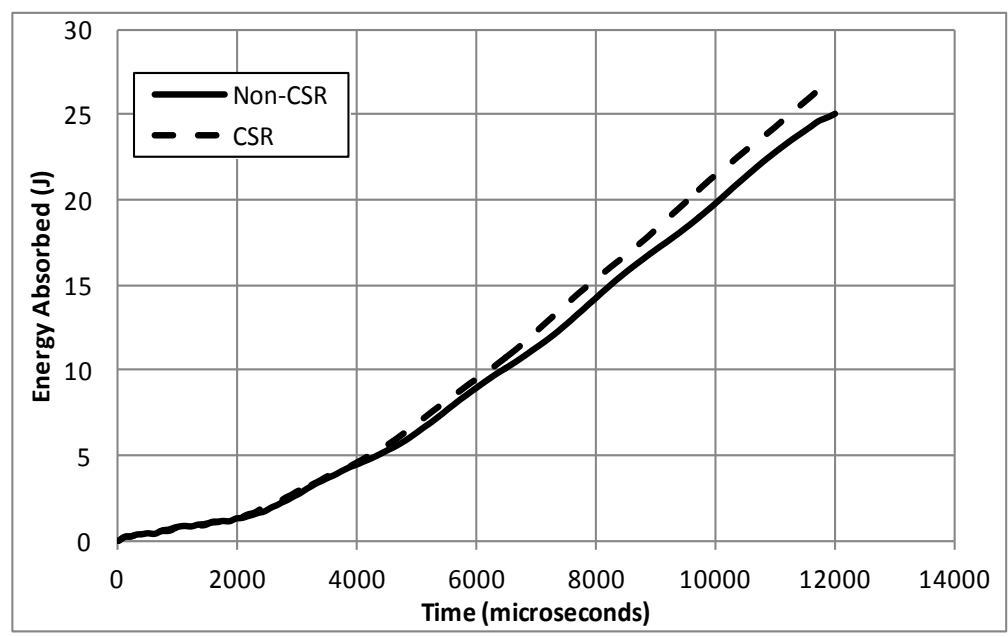

Fig. 14 Total energy absorbed for both composite facesheets during drop-weight impact event

\subsubsection{High Strain Rate (SHPB) Behavior}

The high strain rate behavior of the facesheet and core materials was investigated using a SHPB apparatus. For the dynamic behavior of the facesheet materials, Fig. 15a, it can be observed that the Engineering stress-strain response shows two distinct deformation regions, denoted by (I) and (II), for the behavior of the resin, and the resin/fiber mixture respectively. Due to the fabrication of the composite facesheets there exists plain resin, before the resin/fiber composites. Therefore, as the specimen compresses, you first see the behavior of the resin only, and then the behavior of the overall facesheet (resin/fiber). Since the Non-CSR toughened facesheet exhibit catastrophic failure and excessive damage, only the CSR toughened composite facesheet results will be shown. 


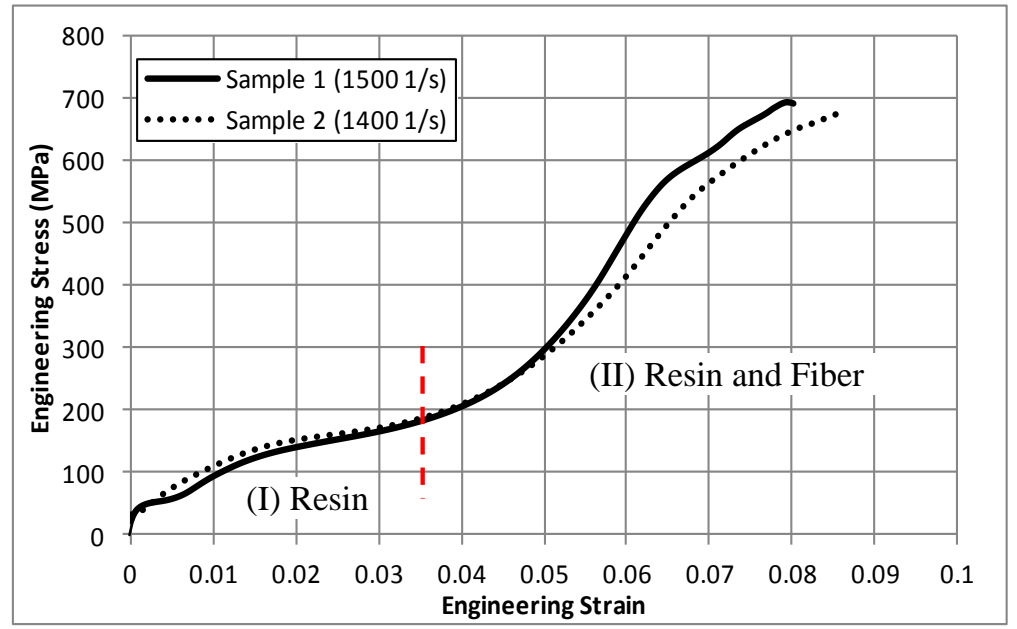

(a) High strain rate behavior of the CSR toughened composite facesheets

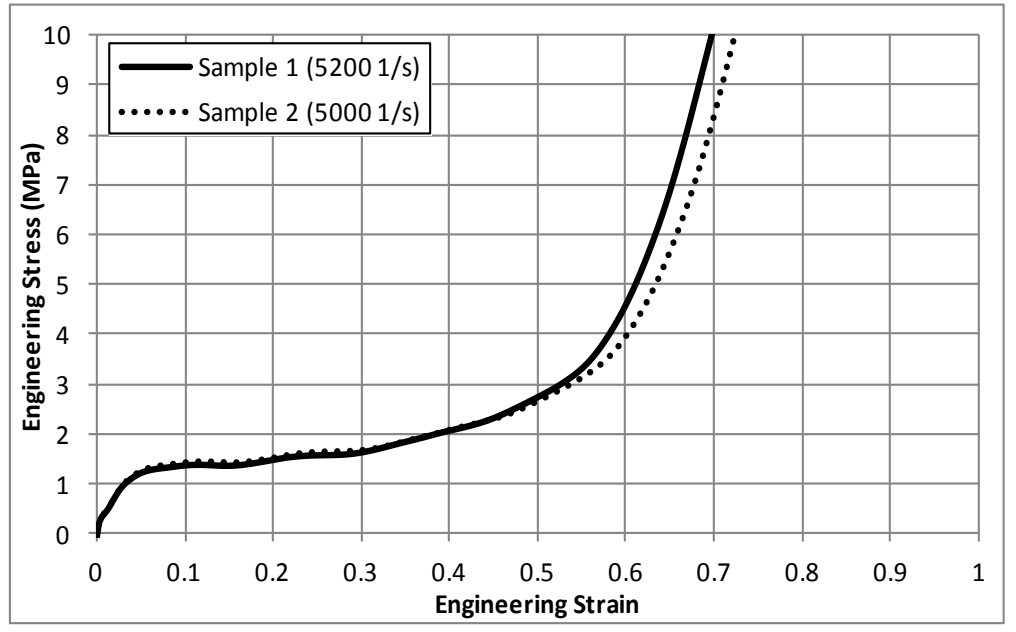

(b) High strain rate behavior of the Corecell ${ }^{\mathrm{TM}}$ A500 foam

Fig. 15 High strain rate compressive behavior of composite facesheets and A500 foam core

Fig. 15b shows the dynamic behavior of the A500 foam core material. Note, the stress-strain curves also show the three deformation regions, as they did under quasistatic loading, even though the densification region is much harder to achieve under dynamic loading conditions. The high strain rate yield stress and plateau (flow) stress of the A500 foam is much higher than its quasi-static yield and plateau stresses, approximately $100 \%$ (1.60 $\mathrm{MPa}$ and $0.88 \mathrm{MPa}$ respectively). The improvement of the 
mechanical behavior from quasi-static to high strain-rates in this core material, as well its long stress plateaus, signifies its ability to absorb large amounts of energy under high strain-rate dynamic loading. Therefore, it shows great potential in being used as a core material in sandwich structures subjected to high intensity air blasts.

\subsection{Blast Response of Core-shell Rubber (CSR) Toughened Sandwich Composites}

\subsubsection{Real-time Deformation}

The real-time observations of the transient behavior for both types of configurations subjected to shock wave loading are shown in Fig. 16. The shock wave (pressure wave) propagates from the right side of the image to the left side and some detailed deformation mechanisms are pointed out in the figures. It should be noted that the time scheme used to represent the images in each configuration is identical. Therefore, for both of the configurations investigated, the images are correlated based on the same time per frame. This allows for a better comparison between the different sandwich panels in regards to the failure mechanisms and extent of damage observed.

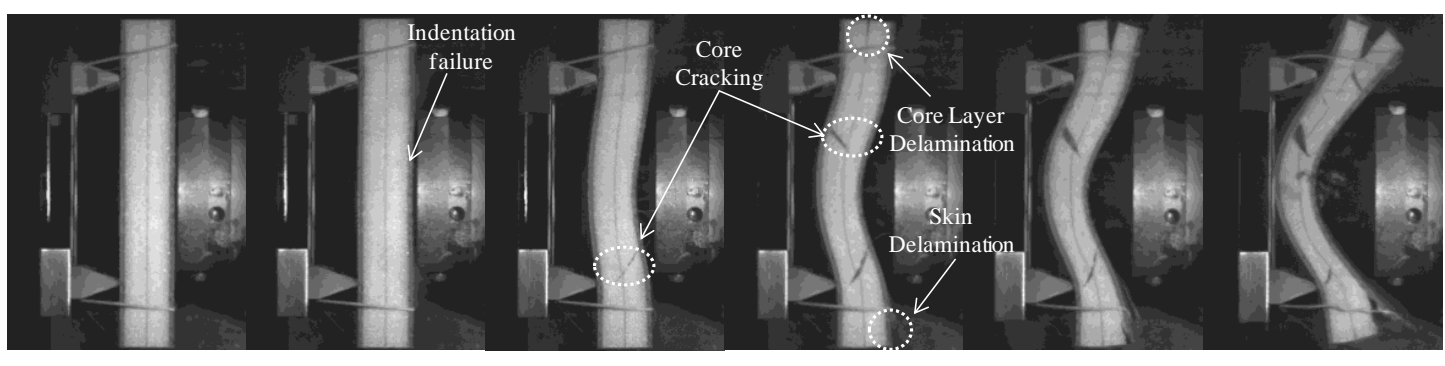

$$
\mathrm{t}=0 \mu \mathrm{s} \quad \mathrm{t}=100 \mu \mathrm{s} \quad \mathrm{t}=400 \mu \mathrm{s} \quad \mathrm{t}=700 \mu \mathrm{s} \quad \mathrm{t}=1000 \mu \mathrm{s} \quad \mathrm{t}=1600 \mu \mathrm{s}
$$

(a) Non-CSR Toughened

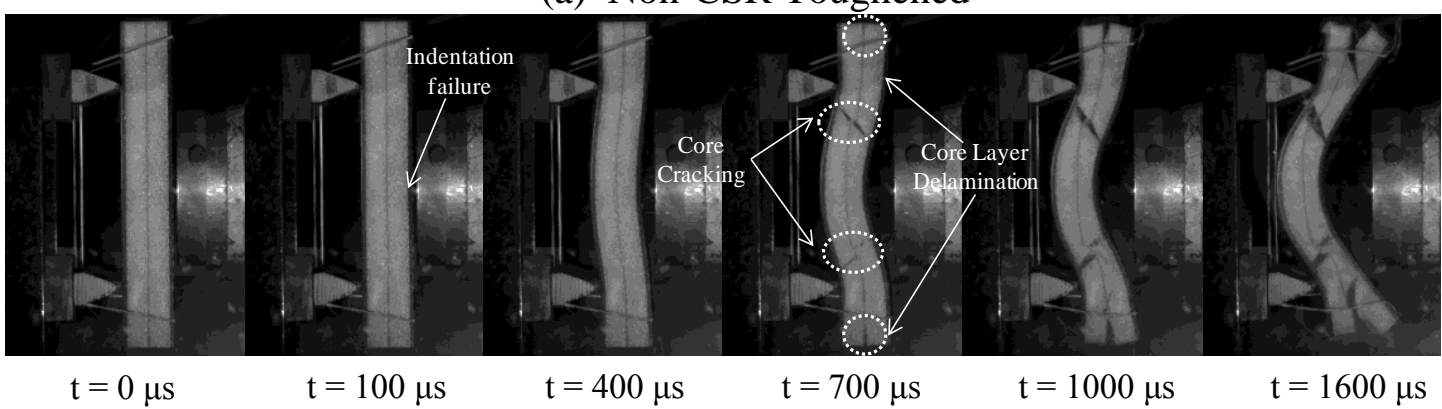

(b) CSR Toughened

Fig. 16 High-speed images for (a) Non-CSR and (b) CSR toughened sandwich composites 


\subsubsection{Deflection}

The mid-point deflection of each sandwich panel and all of its constituents was obtained from the high-speed images and a typical response can be seen in Fig. 17. For both configurations studied, the midpoint deflection of the front face (front skin) and back face (back skin) of the specimen was plotted. For the sandwich composite without core-shell rubber (Non-CSR), as shown in Fig 17a, it is evident that at $\mathrm{t}=$ $1600 \mu$ s the front face deflects to approximately $46 \mathrm{~mm}$, while the back facesheet deflects approximately $35 \mathrm{~mm}$. Therefore, the difference between the deflection of the front face and deflection of the back face signifies the total amount of compression observed in the core. Therefore, it can be concluded that the core compressed approximately $11 \mathrm{~mm}$, which is $30 \%$ of its original thickness $(38 \mathrm{~mm})$.

For the core-shell rubber (CSR) toughened sandwich composite, as shown in Fig $17 \mathrm{~b}$, it is evident that at $\mathrm{t}=1600 \mu \mathrm{s}$ the front face deflects to approximately $45 \mathrm{~mm}$, while the back facesheet deflects approximately $32 \mathrm{~mm}$. Therefore, the difference between the deflection of the front face and deflection of the back face signifies the total amount of compression observed in the core. Therefore, it can be concluded that the core compressed approximately $12 \mathrm{~mm}$, which is $30 \%$ of its original thickness (38 $\mathrm{mm})$.

Fig. 17c shows a comparison between the back face deflections of both configurations. By $\mathrm{t}=1600 \mu \mathrm{s}$, the back facesheet of the Non-CSR toughened sandwich composite deflects to approximately $35 \mathrm{~mm}$, while the back facesheet of the CSR toughened sandwich composite deflects to approximately $32 \mathrm{~mm}$. Therefore, it can be concluded that when using a nano-scale CSR toughened sandwich composite, 
the deflection across the central region of the back facesheet is reduced approximately $8 \%$.

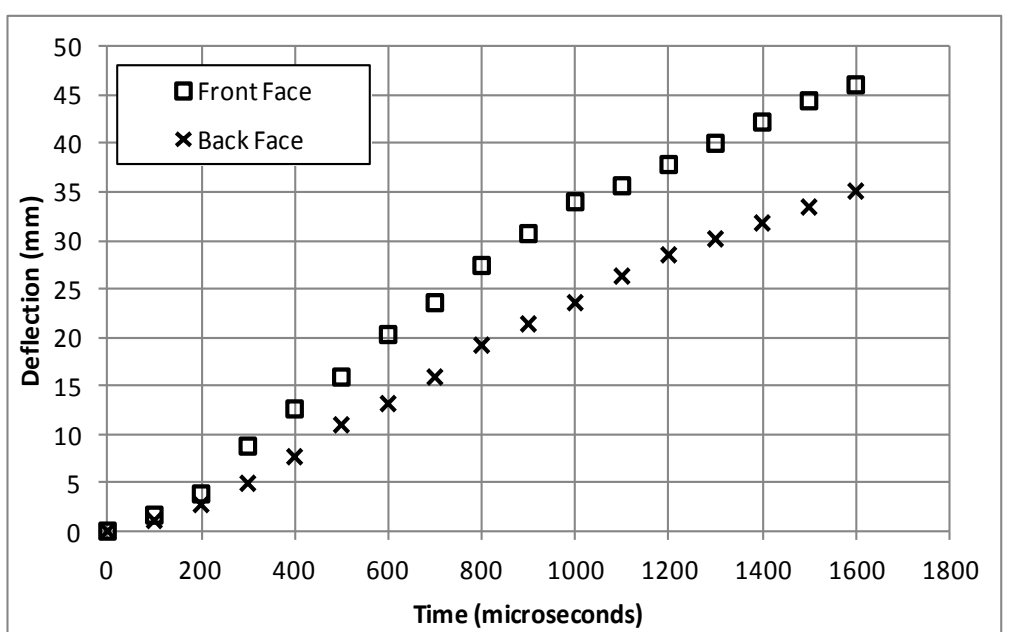

(a) Mid-point deflection of Non-CSR toughened

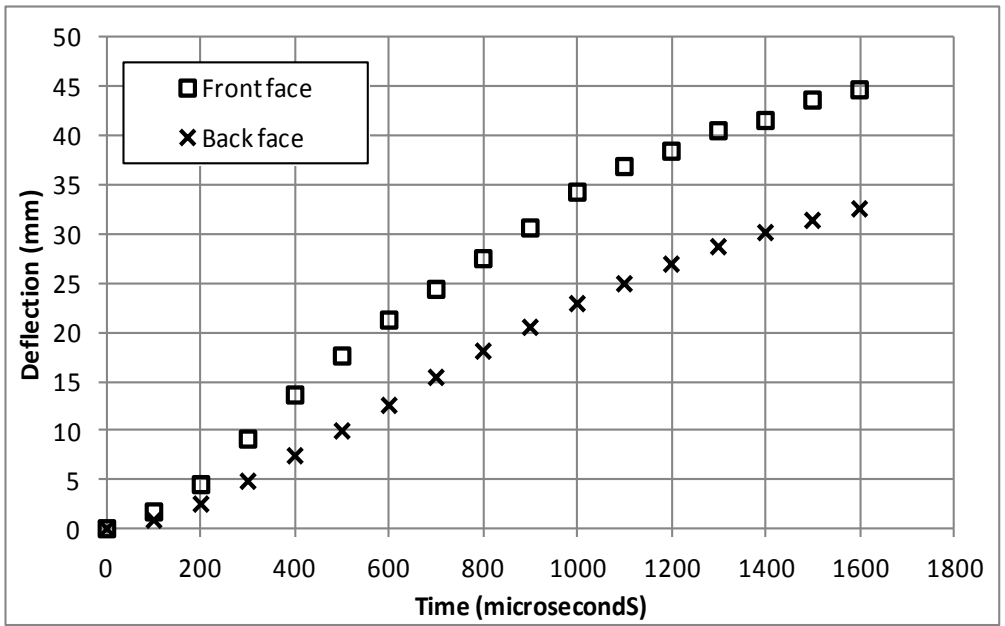

(b) Mid-point deflection of CSR toughened

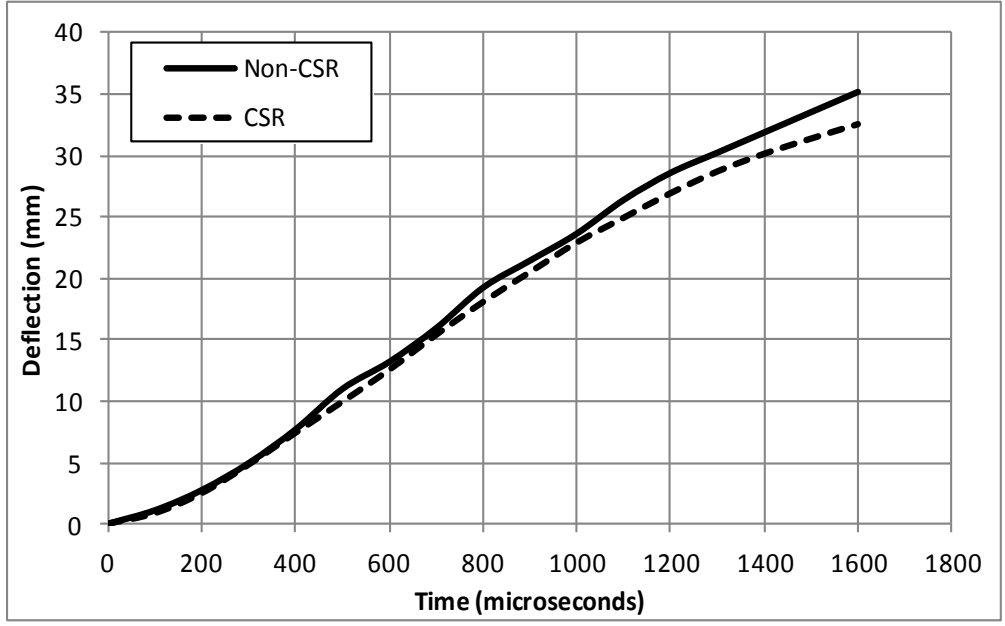

(c) Comparison of back face deflections for both configurations

Fig. 17 Mid-point deflections of both configurations 


\subsubsection{Digital Image Correlation (DIC) Analysis}

Utilizing the Digital Image Correlation (DIC) technique, the full-field deflection (W), in-plane strain $\left(\varepsilon_{\mathrm{yy}}\right)$ and particle velocity $(\mathrm{dW} / \mathrm{dt})$ of the back facesheet of each configuration were generated. Fig. 18 - Fig. 21 show the full-field results for the back facesheet of both configurations respectively. Fig. 18 shows the full-field out-of-plane deflection (W) during the initial fluid-structure interaction ( $\mathrm{t} \leq \sim 250 \mu \mathrm{s}$, [22]), with an emphasis on the shape of the loading, as indicated by the localized areas of larger deflection. For the Non-CSR toughened sandwich composites, as shown in Fig. 18a, by $\sim \mathrm{t}=100 \mu \mathrm{s}$, the loading can be observed as a circular region in the center of the back facesheet. For the CSR toughened sandwich composites, as shown in Figures $18 \mathrm{~b}$, by $\mathrm{t}=100 \mu$ s the loading is more dispersed across the back facesheet, resulting in up to two or even three areas of localized deflection (loading). Therefore, it can be concluded that utilizing CSR, and thus introducing nano-scale rubber particles into the composites, aids in dispersing the initial loading on the structure, resulting in up to three areas of localized deflection (loading) on the back facesheets. 


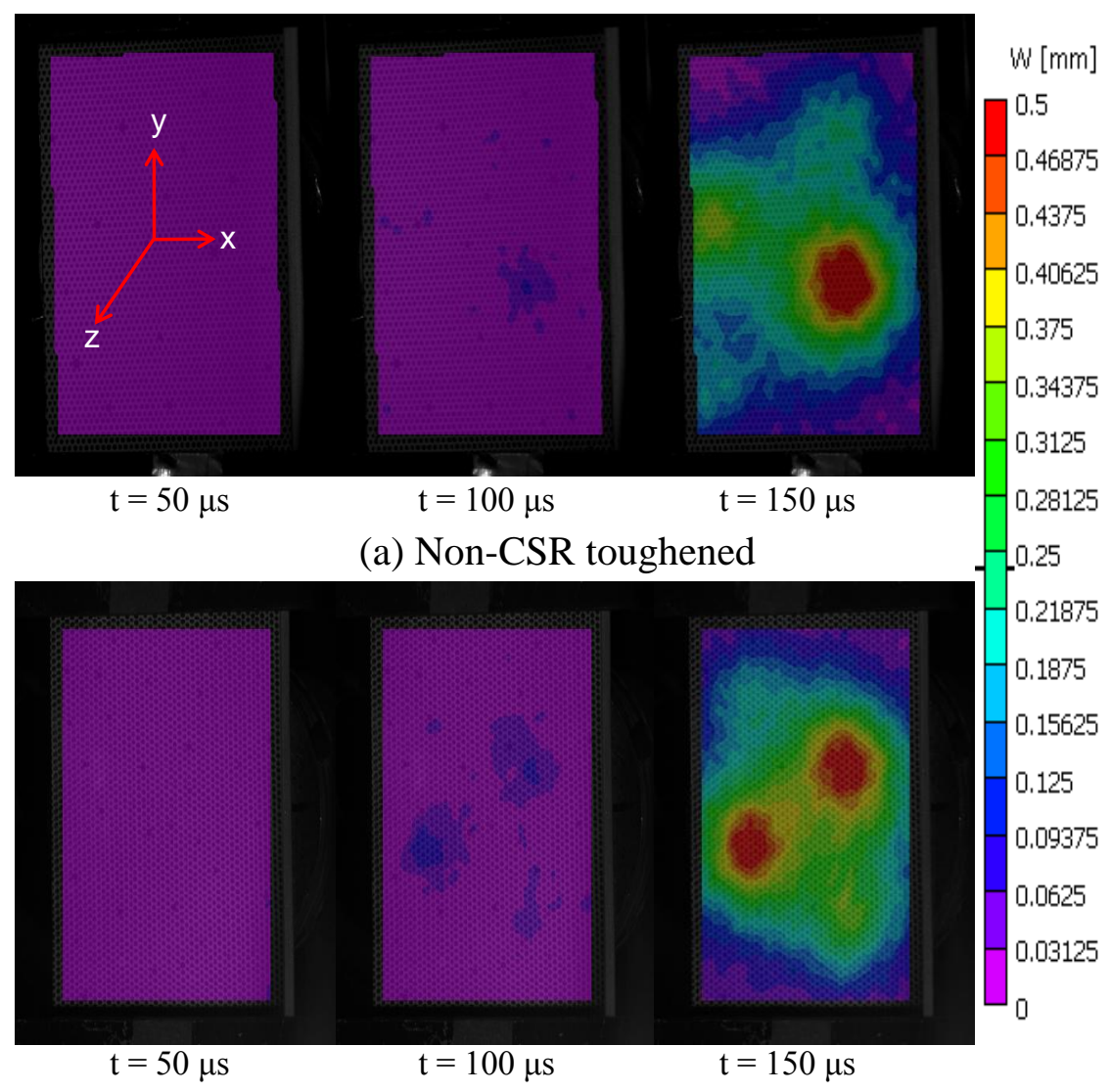

(b) CSR toughened

Fig. 18 Localized areas of larger deflections (loading) during fluid-structure interaction

Fig. 19 shows the full-field out-of-plane deflection (W) for both configurations. Note the scale ranges from $0 \mathrm{~mm}$ (purple) to $35 \mathrm{~mm}$ (red). It is evident from the figure that the back face of both core configurations exhibits limited out-of-plane deflection prior to $\mathrm{t}=400 \mu \mathrm{s}$. Between $\mathrm{t}=400 \mu \mathrm{s}$ and $\mathrm{t}=1600 \mu \mathrm{s}$, both of these configurations continue to bend and exhibit deflections. For the Non-CSR toughened sandwich composite, as shown in Fig. 19a, it can be observed that at $t=1600 \mu$ s, the central region of the panel has deflected approximately $35 \mathrm{~mm}$. When using CSR toughened sandwich composites (Fig. 19b), it can be seen that at $t=1600 \mu \mathrm{s}$, the central region of the panel has deflected approximately $32 \mathrm{~mm}$. Therefore, it can be concluded that 
when using a nano-scale CSR toughened sandwich composite, the deflection across the central region of the back facesheet is reduced approximately $8 \%$.

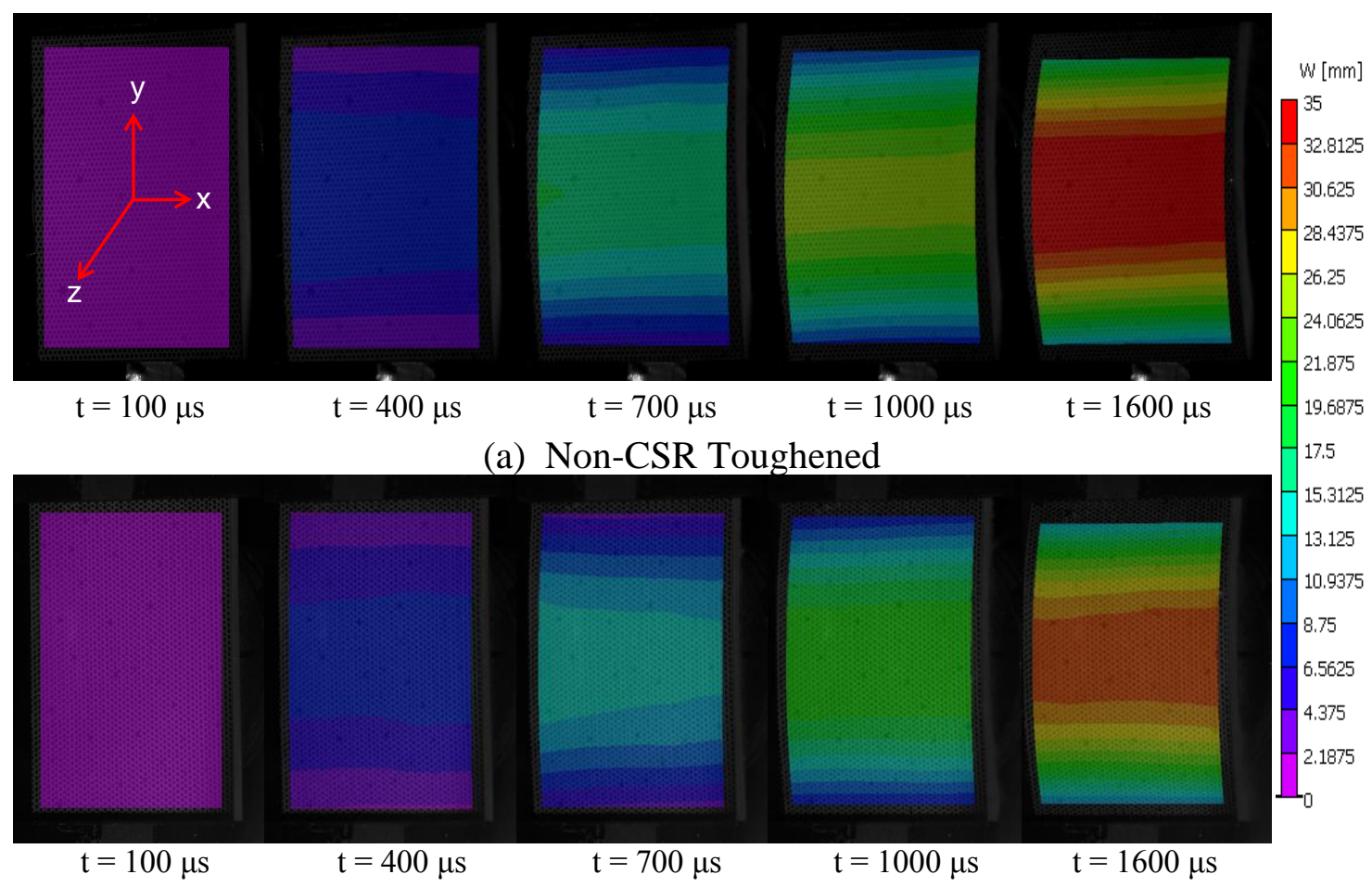

(b) CSR Toughened

Fig. 19 Full-field out-of-plane deflection (W) of both

The full-field, in-plane strain $\left(\varepsilon_{\mathrm{yy}}\right)$ is shown in Fig 20 for both configurations with a scale of 0 (purple) to .025 (red), or $0 \%$ to $2.5 \%$ respectively. It can be observed in the figure that the back face of both core configurations exhibits very minimal in-plane strain $\left(\varepsilon_{y y}\right)$ prior to $t=100 \mu \mathrm{s}$. Between $\mathrm{t}=100 \mu \mathrm{s}$ and $\mathrm{t}=1600 \mu \mathrm{s}$, both of these configurations continue bending and the in-plane strain values continue to increase. For the Non-CSR toughened sandwich composite, as shown in Fig. 20a, it can be observed that at $\mathrm{t}=1600 \mu \mathrm{s}$, the central region of the panel exhibits a maximum inplane strain value of $2.5 \%$. When using CSR toughened sandwich composites (Fig. 20b), it can be seen that at $\mathrm{t}=1600 \mu \mathrm{s}$, the central region of the panel exhibits a maximum in-plane strain value of $2.0 \%$. Therefore, it can be concluded that when 
using a nano-scale CSR toughened sandwich composite, the maximum in-plane strain across the central region of the back facesheet is reduced approximately $20 \%$.

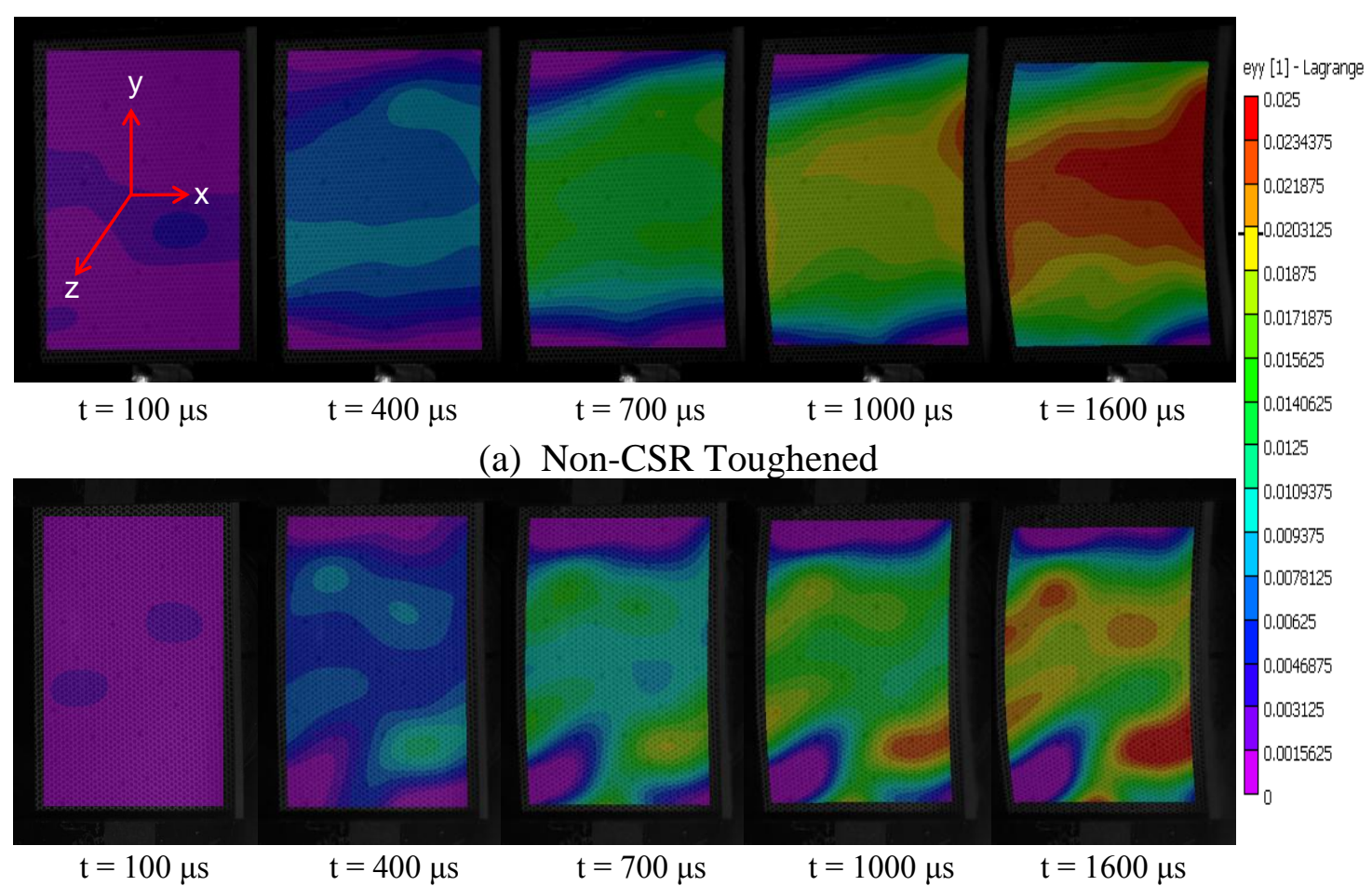

(b) CSR Toughened

Fig. 20 Full-field in-plane strain $\left(\varepsilon_{\mathrm{yy}}\right)$ of both configurations

Fig. 21 shows the full-field out-of-plane velocity (dW/dt) for both configurations. Note the scale ranges from $0 \mathrm{~mm} / \mathrm{s}$ (purple) to $32,000 \mathrm{~mm} / \mathrm{s}$ (red), or $0 \mathrm{~m} / \mathrm{s}$ to $32 \mathrm{~m} / \mathrm{s}$. For the Non-CSR toughened sandwich composites, as shown in Fig. 21a, the back face exhibits a large out-of-plane velocity $(\sim 50 \%$ of its maximum) by $\mathrm{t}=100 \mu \mathrm{s}$. At $\mathrm{t}=$ $400 \mu \mathrm{s}$, the central region of the back face has reached an out-of-plane velocity of approximately $30 \mathrm{~m} / \mathrm{s}$. By $\mathrm{t}=700 \mu$ s the velocity has reached a maximum value of 32 $\mathrm{m} / \mathrm{s}$, and reduced back to approximately $30 \mathrm{~m} / \mathrm{s}$. From $\mathrm{t}=700 \mu \mathrm{s}$ and onward, the velocity continues to decrease from $30 \mathrm{~m} / \mathrm{s}$ to $24 \mathrm{~m} / \mathrm{s}(\mathrm{t}=1000 \mu \mathrm{s})$ and finally down to $15 \mathrm{~m} / \mathrm{s}(\mathrm{t}=1600 \mu \mathrm{s})$. For the CSR toughened sandwich composites, as shown in Fig. 
$21 \mathrm{~b}$, the back face exhibits a smaller out-of-plane velocity ( $40 \%$ of its maximum) by $\mathrm{t}=100 \mu \mathrm{s}$. At $\mathrm{t}=400 \mu \mathrm{s}$, the central region of the back face has reached an out-ofplane velocity of approximately $25 \mathrm{~m} / \mathrm{s}$. By $\mathrm{t}=700 \mu$ s the velocity has reached a maximum values, $28 \mathrm{~m} / \mathrm{s}$, and reduced to $26 \mathrm{~m} / \mathrm{s}$. From $\mathrm{t}=700 \mu \mathrm{s}$ and onward, the velocity continues to decrease from $26 \mathrm{~m} / \mathrm{s}$ to $21 \mathrm{~m} / \mathrm{s}(\mathrm{t}=1000 \mu \mathrm{s})$ and finally down to $12 \mathrm{~m} / \mathrm{s}(\mathrm{t}=1600 \mu \mathrm{s})$.
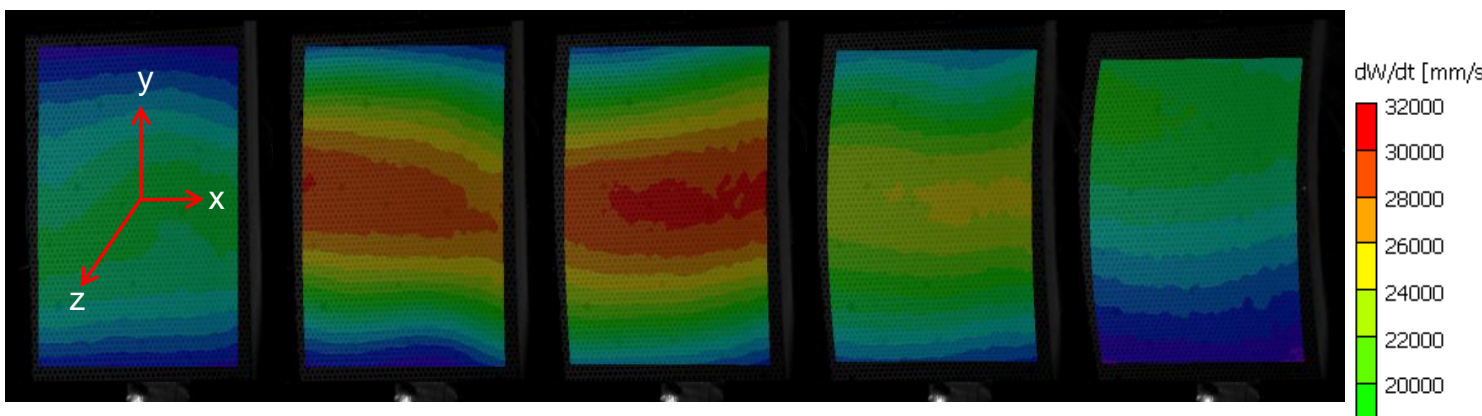

$\mathrm{t}=100 \mu \mathrm{s}$

$\mathrm{t}=400 \mu \mathrm{s}$

$\mathrm{t}=700 \mu \mathrm{s}$

$\mathrm{t}=1000 \mu \mathrm{s}$

$\mathrm{t}=1600 \mu \mathrm{s}$

18000

(a) Non-CSR Toughened

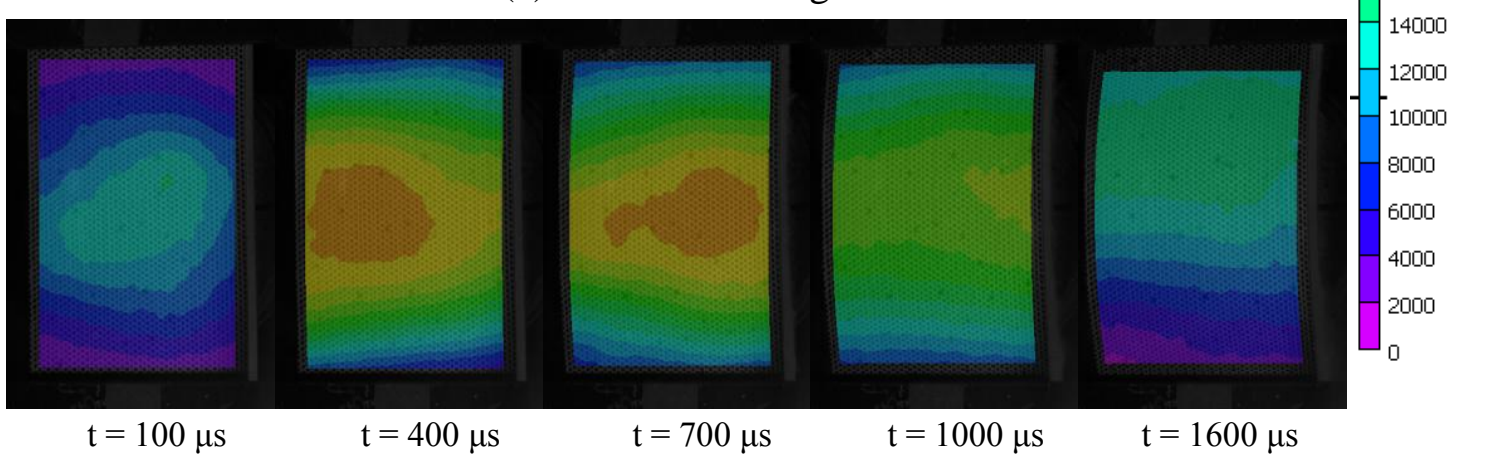

(b) CSR Toughened

Fig. 21 Full-field out-of-plane velocity (dW/dt) of both configurations

Therefore, it can be concluded that when using a nano-scale CSR toughened sandwich composites, the maximum back face velocity is approximately $12 \%$ smaller than the Non-CSR toughened sandwich composites. Also note, the velocity of the panel at $\mathrm{t}=1600 \mu \mathrm{s}$ is approximately $20 \%$ smaller as well, i.e. $15 \mathrm{~m} / \mathrm{s}$ and $12 \mathrm{~m} / \mathrm{s}$ respectively. 
Using the point inspection tool from the Digital Image Correlation (DIC) software, a point directly in the center of the back face of each specimen was chosen. The out-of-plane deflection (W) showed excellent agreement with the results generated utilizing the high-speed images and therefore, only the in-plane strain $\left(\varepsilon_{\mathrm{yy}}\right)$ and out-of-plane velocity (dW/dt) results are shown. Fig. 22 and Fig. 23 show the inplane strain and out-of-plane velocity values obtained. Looking at the in-plane strain values (Fig. 22) it can be seen that at $\mathrm{t}=1600 \mu \mathrm{s}$, the maximum in-plane strain value at the central point of the back facesheet for the Non-CSR toughened sandwich composite is approximately 0.025 , or $2.5 \%$. When using a CSR toughened sandwich composite, it can be seen that at $\mathrm{t}=1600 \mu \mathrm{s}$, the maximum in-plane strain value at the central point of the back facesheet is approximately 0.020 , or $2.0 \%$. Therefore, it can be concluded that when using CSR toughened sandwich composites, the maximum inplane strain across the central region of the back facesheet is reduced $20 \%$ in comparison to the sandwich composites with equivalent core layer mass.

Fig. 23 shows the out-of-plane velocity (dW/dt) for both configurations. For the Non-CSR toughened sandwich composite, the maximum velocity is reached at $t=600$ $\mu \mathrm{s}$ and is approximately $32 \mathrm{~m} / \mathrm{s}$. For the CSR toughened sandwich composite, the maximum velocity is reached at approximately the same time, $\mathrm{t}=600 \mu \mathrm{s}$, but the velocity is larger $(34 \mathrm{~m} / \mathrm{s})$. Therefore, it can be concluded that when nano-scale CSR toughened sandwich composites, the maximum out-of-plane velocity across the central region of the back facesheet is reduced $12 \%$ in comparison to the sandwich composites with equivalent core layer mass. 


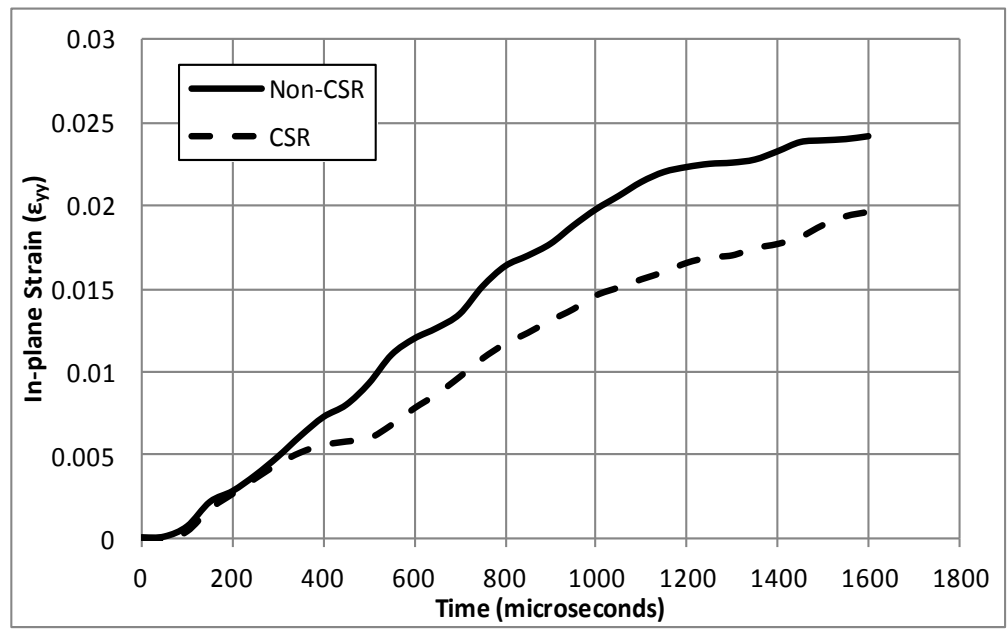

Fig. 22 In-plane strain $\left(\varepsilon_{\mathrm{yy}}\right)$ of both configurations

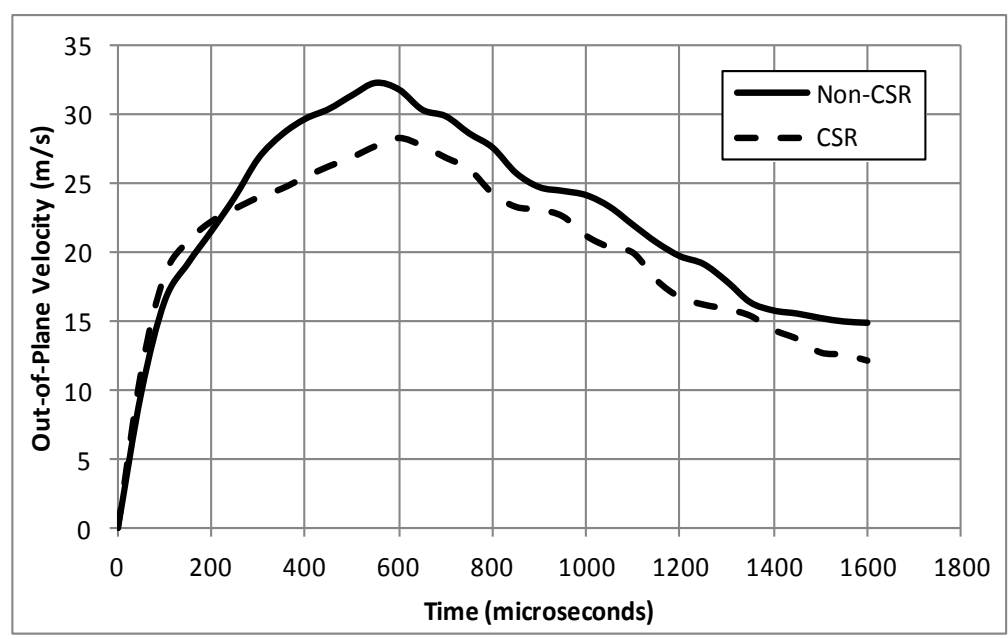

Fig. 23 Out-of-plane velocity (dW/dt) of both configurations

In order to provide a more comprehensive understanding on the behavior response of the sandwich panels during the shock wave loading, the individual facesheets were subjected to blast wave loading as well. The Digital Image Correlation (DIC) technique was applied to the facesheet experiments (incident peak pressure 0.3 $\mathrm{MPa})$. For this investigation full-field analysis was also carried out, as well as lineinspection. Since the behavior of the facesheets during the initial fluid structure interaction was similar to that of the sandwich composites, only the line-inspection analysis will be presented here. Utilizing the line-inspection tool from the Digital 
Image Correlation (DIC) software, a longitudinal line directly in the center of the back face of each specimen was chosen, and the results were extracted, as shown in Fig. 24. It can be observed from the figure that by $\mathrm{t}=3200 \mu \mathrm{s}$, the Non-CSR toughened sandwich composite exhibits a crack across the central region. Also note, the amount of bending in the Non-CSR composite facesheets is higher than the CSR toughened facesheet.

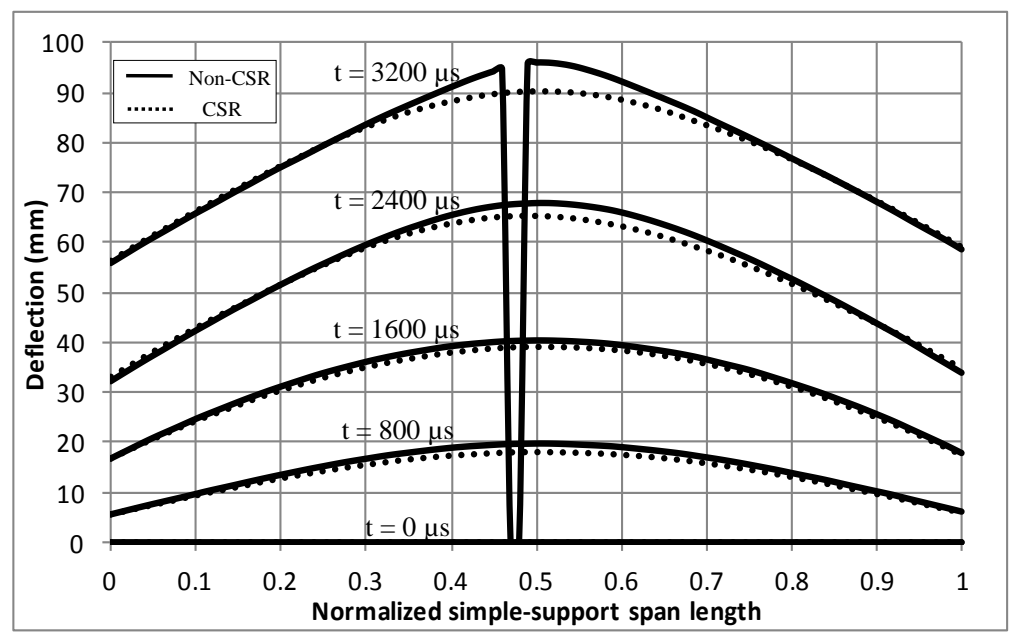

Fig. 24 Longitudinal line-inspection analysis across central region of back face for both composite facesheet systems

\subsubsection{Residual Compressive Strength}

After the blast loading event occurred on the facesheets, both types of composite facesheets were subjected to post-blast residual compressive strength measurements. For each composite panel system, at least two samples were tested. A typical response is shown in Fig. 25. It can be observed that after the blast loading event, the Non-CSR toughened composite facesheet achieved a maximum residual strength of $15 \mathrm{MPa}$, at a strain level of approximately $1 \%$. Following this, the composite facesheet exhibited brooming due to cracking and heavy fiber delamination (Fig. 25b). 


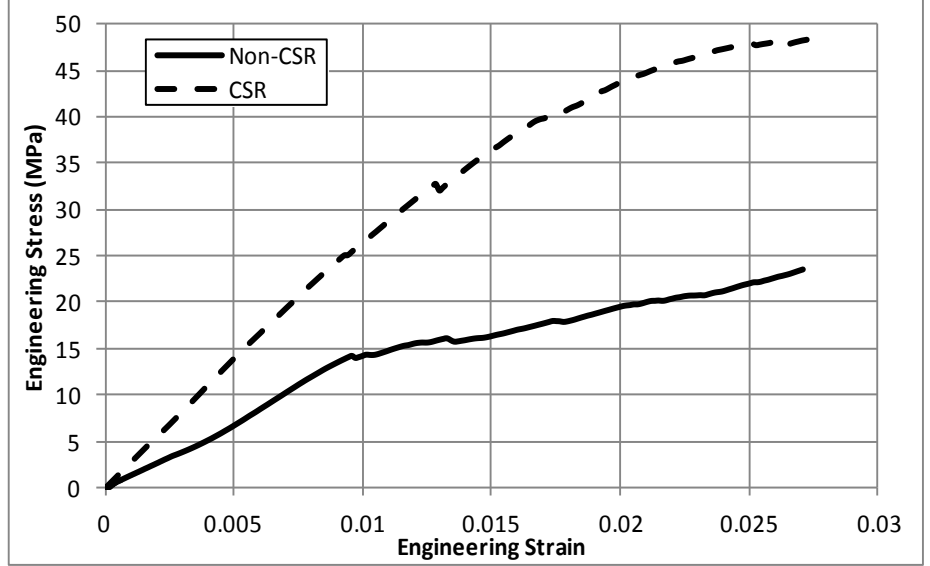

(a) Residual compressive strengths of both types of composite facesheets

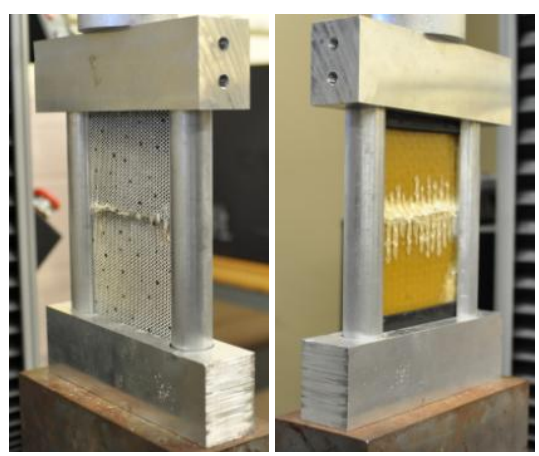

(b) Non-CSR Toughened

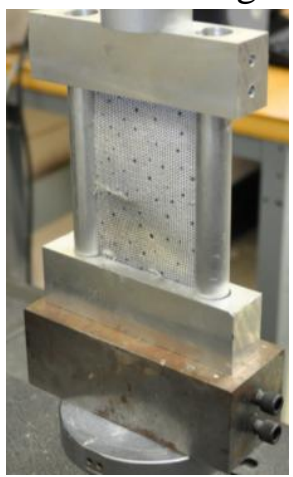

(c) CSR Toughened

Fig. 25 Residual compressive strength of both types of composite facesheets after being subjected to high intensity blast loading (incident peak pressure $0.3 \mathrm{MPa}$ )

For the CSR toughened composite facesheet, this panel achieved a maximum post-blast residual strength of $48 \mathrm{MPa}$, at a strain level of approximately $2.5 \%$. Therefore, it can be concluded that the addition of nano-scale CSR particles to the composite facesheet allows for a post-blast residual strength which is approximately $300 \%$ higher than that of the Non-CSR toughened facesheet.

\subsubsection{Post-mortem Analysis}

After the quasi-static (Drop-weight) and dynamic events (SHPB and Shock Tube) occurred, the damage patterns were visually examined and recorded using a high resolution digital camera and are shown in Fig. 26 - Fig. 29. The damage patterns of both types of composite facesheets subjected to a $150 \mathrm{~J}$ drop-weight impact event are 
shown in Fig. 26. For the Non-CSR toughened composite facesheet, heavy fiber delamination can be observed along the central region of the facesheet (Fig. 26a). For the CSR toughened composite facesheet, as shown in Fig. 26b, very little damage can be observed.

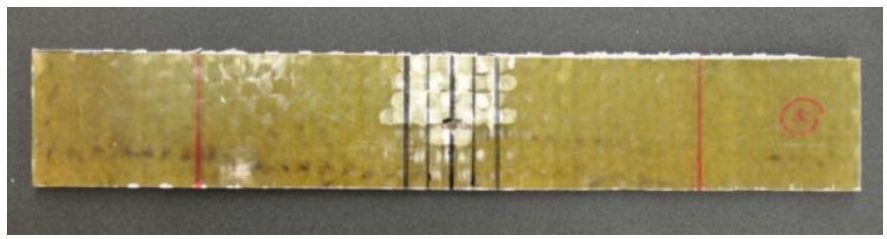

(a) Non-CSR Toughened

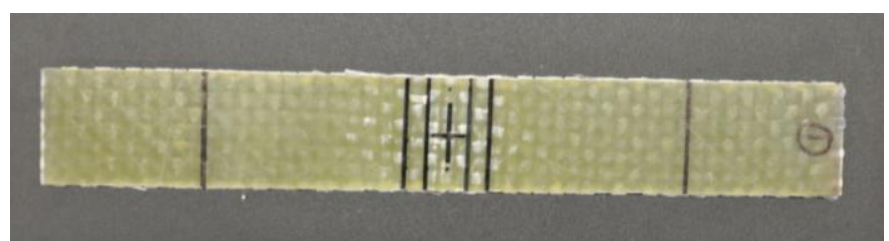

(b) CSR Toughened

Fig. 26 Visual examination of both composite facesheets after being subjected to $150 \mathrm{~J}$ impact

Fig. 27 shows the damage patterns of composite facesheets subjected to a strain rate of $10^{3}(5000$ 1/s) using a Split Hopkinson Pressure Bar apparatus. It can be observed from the figure that the CSR toughened composite facesheet (Fig. 27b) maintained its structural integrity, while the Non-CSR toughened composite facesheet (Fig. 27a) did not (catastrophic failure).

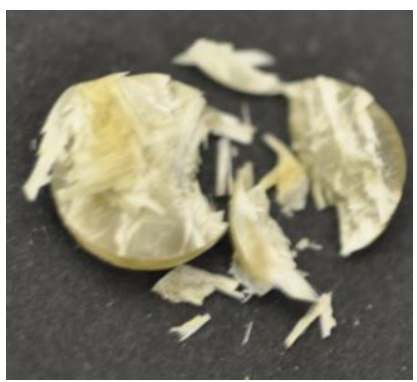

(a) Non-CSR Toughened

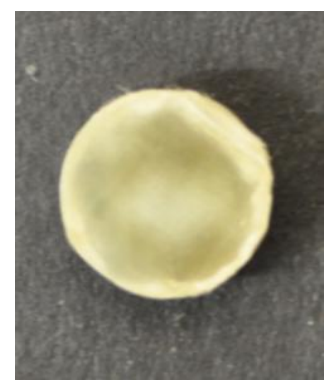

(b) CSR Toughened

Fig. 27 Visual examination of both composite facesheets after being subiected to high strain-rate loading (SHPB) 
The damage patterns of both types of sandwich composite panels subjected to an incident peak pressure of 1.0 $\mathrm{MPa}$, a reflected peak pressure of 5.0 $\mathrm{MPa}$, and an incident velocity of $1000 \mathrm{~m} / \mathrm{s}$ using the shock tube apparatus are shown in Fig. 28. When the Non-CSR toughened sandwich composite was subjected to high-intensity loading, as shown in Fig. 28a, the damage was confined to the areas where the supports were located in the shock tube and core cracking is visible in these two areas. The core cracks propagated completely through the foam core. Core delamination is visible between the two core layers of A500 foam. Also one of the core cracks lead to back skin delamination, where the core separated from the back facesheet. Some core compression is visible in the first core layer of A500 foam. Also heavy fiber delamination and cracking is visible along the front facesheet.

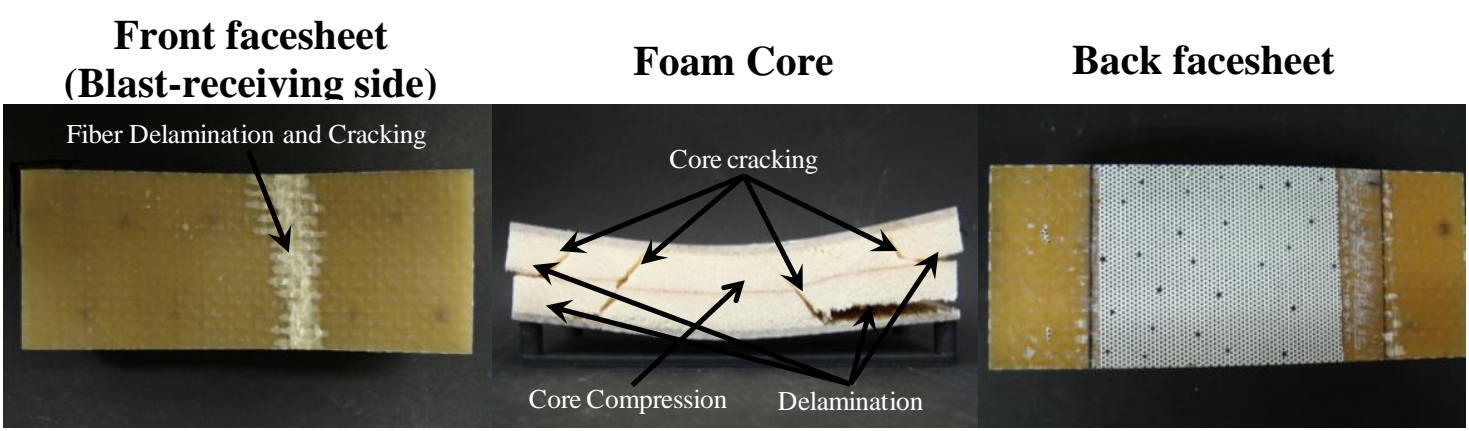

(a) Non-CSR Toughened

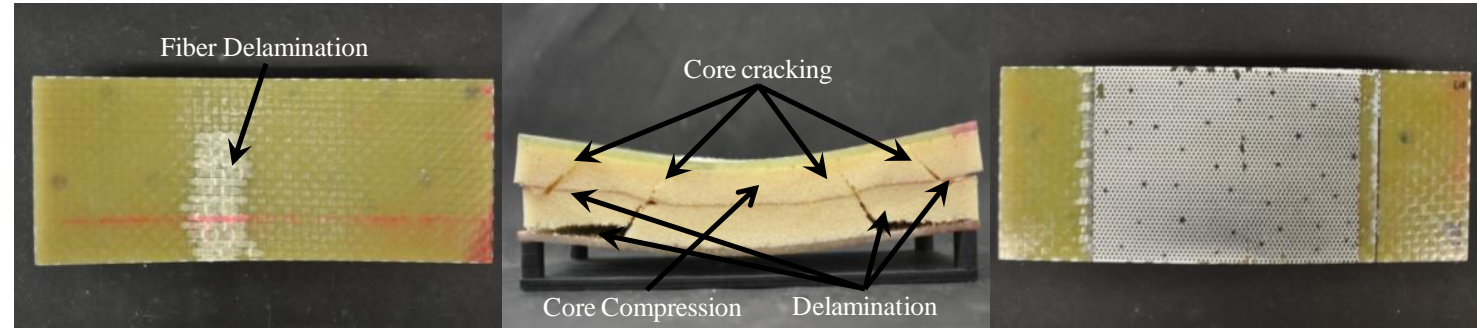

(b) CSR Toughened

Fig. 28 Visual examination of both configurations after being subjected to high intensity blast load (incident peak pressure $1.0 \mathrm{MPa}$ 
When the CSR toughened sandwich composite was subjected to high-intensity loading, as shown in Fig. 28b, the damage was again confined to the areas where the supports were located in the shock tube and core cracking is visible in these two areas. The core cracks propagated completely through the foam core. Core delamination is visible between the two core layers of A500 foam. Also, the core cracks lead to back skin delamination, where the core separated from the back facesheet. Some core compression is visible in the first core layer of A500 foam. Also, fiber delamination is visible along the front facesheet.

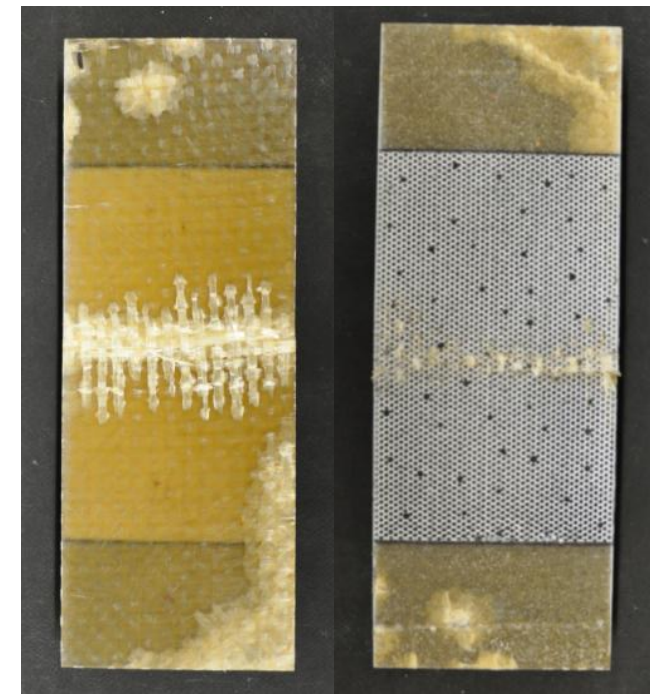

(a) Non-CSR Toughened Composite; (L) Front facesheet, (R) Back facesheet

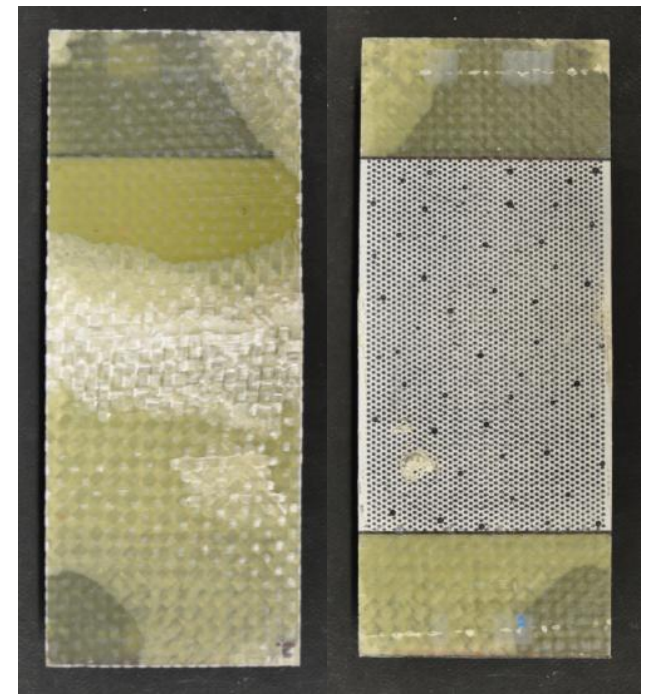

(b) CSR Toughened Composite; (L) Front facesheet, (R) Back facesheet

Fig. 29 Visual examination of both composite facesheets after being subjected to high intensity blast loading (incident peak pressure of $0.3 \mathrm{MPa}$ )

Fig. 29 shows the damage patterns of both types of composite facesheets subjected to an incident peak pressure of $0.3 \mathrm{MPa}$, a reflected peak pressure of 1.0 $\mathrm{MPa}$, and an incident velocity of $650 \mathrm{~m} / \mathrm{s}$ using the shock tube apparatus. When the Non-CSR toughened facesheet was subjected to the blast loading, heavy fiber delamination and a large central crack was observed, as shown in Fig. 29a. When the 
CSR toughened facesheet was subjected to the blast loading, heavy delamination was observed, as shown in Fig. 29b.

The results of the post-mortem analysis, Fig. 26 - Fig. 29, show that using a coreshell rubber (CSR) toughened epoxy system allows for a more impact resistant composite system, and aids in dispersing the high-intensity dynamic loadings. The CSR toughened panels exhibited heavy amounts of delamination, but the structural integrity was maintained. On the contrary, the Non-CSR toughened sandwich panels exhibited heavy fiber delamination and cracking. For the Non-CSR toughened composite facesheets, these panels failed catastrophically and structural integrity was lost.

\section{Conclusions}

The following is the summary of the investigation:

(1) Core-shell rubber particles (CSR) have a significant effect on the quasi-static and dynamic behavior of composite materials and sandwich structures. It was observed that the addition of $4 \%$ CSR to E-glass Vinyl-Ester (EVE) composite facesheets increased the tensile strength $(\sim 10 \%)$ and drop-weight impact resistance $(\sim 10 \%)$,. After the dynamic loading, such as SHPB and shock wave loading, the CSR toughened facesheets maintained structural integrity, while the Non-CSR facesheets did not.

(2) The dynamic stress-strain response is significantly higher than the quasi-static response for the Corecell ${ }^{\mathrm{TM}}$ A500 foam studied. The increase in the yield strength from quasi-static response to dynamic response, along with the longer 
stress plateau, indicates that this core material shows great potential in absorbing large amounts of energy.

(3) Two types of sandwich composites were fabricated, one with the addition of nano-scale core-shell rubber (CSR) particles during infusion, and one without (Non-CSR). The core material and thickness, as well as overall specimen dimensions were held constant. Results indicated that the addition of nanoscale core-shell rubber (CSR) particles to the panels allows for an increase in blast performance. By dispersing the initial loading, the CSR toughened sandwich composites exhibit lower amounts of out-of-plane deflection and velocity, as well as in-plane strain, approximately $8 \%, 12 \%$ and $20 \%$ respectively.

\section{Acknowledgements}

The authors kindly acknowledge the financial support provided by Dr. Yapa D. S. Rajapakse, under Office of Naval Research Grant No. N00014-10-1-0662. The authors acknowledge the support provided by the Department of Homeland Security under Cooperative Agreement No. 2008-ST-061-ED0002. Authors thank Gurit SP Technology for providing the materials, as well as Dr. Stephen Nolet and TPI Composites for providing the facility for creating the composites used in this study. A special thanks to Dr. Richard Brown for providing the Scanning Electron Microscopy (SEM) facility used in obtaining the images of the cell microstructures.

\section{References}

[1] Z. Xue, J.W. Hutchinson, Preliminary assessment of sandwich plates subject to blast loads, Int. J. Mech. Sci. 45 (2003) 687-705. 
[2] N.A. Fleck, V.S. Deshpande, The resistance of clamped sandwich beams to shock loading, J. Appl. Mech. 71 (2004) 386-401.

[3] K.P. Dharmasena, H.N.G. Wadley, Z. Xue, J.W. Hutchinson, Mechanical response of metallic honeycomb sandwich panel structures to high-intensity dynamic loading, Int. J. Imp. Eng. 35 (2008) 1063-1074.

[4] F. Zhu, L. Zhao, G. Lu, Z. Wang, Deformation and failure of blast loaded metallic sandwich panels - Experimental investigations. Int. J. Imp. Eng. 35 (2008) 937-951.

[5] G.N. Nurick, G.S. Langdon, Y. Chi, N. Jacob, Behavior of sandwich panels subjected to intense air blast: part 1- Experiments, Comp. Struct. 91 (2009) 433 - 441.

[6] V.L. Tagarielli, V.S. Deshpande, N.A. Fleck, The high strain rate response of PVC foams and end-grain balsa wood, Compos. B. 39 (2008) 83-91.

[7] D.D. Radford, G.J. McShane, V.S. Deshpande, N.A. Fleck, The response of clamped sandwich plates with metallic foam cores to simulated blast loading, Int. J. Sol. Struct. 44 (2006) 6101-612.

[8] G.J. McShane, V.S. Deshpande, N.A. Fleck, The underwater blast resistance of metallic sandwich beams with prismatic lattice cores, J. Appl. Mech. 74 (2007) 352 364.

[9] G.J. McShane, D.D. Radford, V.S. Deshpande, N.A. Fleck, The response of clamped sandwich plates with lattice cores subjected to shock loading, Euro. J. Mech. - A: Sol. 25 (2006) 215-229.

[10] A.J. Kinloch, S.J. Shaw, D.A. Tod, D.L. Hunston, Deformation and fracture behavior of a rubber-toughened epoxy: 1. Microstructure and fracture studies. Poly. 24 (1983) 1341- 1354.

[11] A.F. Yee, R.A. Pearson, Toughening mechanisms in elastomer-modified epoxies, J. Mater. Sci. 21 (1986) 2462-2474.

[12] C.B. Bucknall, I.K. Partridge, Phase separation in epoxy resins containing polyethersulphone, Poly. 24 (1983) $639-644$. 
[13] A.J. Kinloch, M.L. Yuen, S.D. Jenkins SD (1994) Thermoplastic-toughened epoxy polymers, J. Mater. Sci. 29 (1994) 3781-3790.

[14] B.B. Johnsen, A.J. Kinloch, A.C. Taylor, Toughness of syndiotactic polystyrene/epoxy polymer blends: microstructure and toughening mechanisms, Poly. 46 (2005) 7352-7369.

[15] L.J. Broutman, S. Sahu, The effect of interfacial bonding on the toughness of glass filled polymers, Mater. Sci. Eng. 8 (1971) 98-107.

[16] J. Spanoudakis, R.J. Young, Crack propagation in a glass particle- filled epoxy resin - Part 1, J. Mater. Sci. 19 (1984) 473-486.

[17] L.M. McGrath, R.S. Parnas, J.L. Lenhart, S. King, Fracture toughness of aluminaepoxy composites, Poly. Mater. Sci. Eng. Preprints, 94 (2006) 683 - 684.

[18] R. Griffiths, D. Holloway, The fracture energy of some epoxy resin materials, J. Mater. Sci. 5 (1970) 302-307.

[19] T.H. Hsieh, A.J. Kinloch, K. Masania, J. Sohn Lee, A.C. Taylor, S. Sprenger, The toughness of epoxy polymers and fiber composites modified with rubber microparticles and silica nanoparticles, J. Mater. Sci. 45 (2010) $1193-1210$.

[20] D.G. Dixon, S.J. Harris, M. Dempster, P. Nicholls, Effect of PEEK fibers and powder on joints made with high temperature adhesive, J. Adhes. 65 (1998) 131-162.

[21] A.J. Kinloch, A.C. Taylor, The toughening of cryanate-ester polymers -Part I Physical modification using particles, fibers and woven mats, J. Mater. Sci. 37 (2002) 433-460.

[22] K.F. Lin, Y.D. Shieh, Core-Shell Particles Designed for Toughening the Epoxy Resins: I. Preparation and Characterization of Core-Shell Particles, J. Appl. Poly. Sci. 69 (1998) 2069-2078.

[23] K.F. Lin, Y.D. Shieh, Core-shell particles designed for toughening the epoxy resins: II. Core-shell particle toughened epoxy resins, J. Appl. Poly. Sci. 70 (1998) $2313-2322$. 
[24] L. Becu, A. Maazouz, H. Sautereau and J.F. Gerard, Fracture behavior of epoxy polymers modified with core-shell rubber particles, J. Appl. Poly. Sci. 65 (1997) 24192431.

[25] C. Yan, K.Q. Xiao, L. Ye, Y.W. Mai, Numerical and experimental studies on the fracture behavior of rubber toughened epoxy in bulk specimen and laminated composites, J. Mater. Sci. 37 (2002) 921-927.

[26] G. Giannakopoulos, K. Masania, A.C. Taylor, Toughening of epoxy using coreshell particles, J. Mater. Sci. 46 (2011) 327-338.

[27] Kaneka Texas Corporation, http://www.kanekatexas.com/MX (Accessed September 20, 2011).

[28] A.J. Kinloch, R.D. Mohammed, A.C. Taylor, S. Sprenger, D. Egan, The interlaminar toughness of carbon-fiber reinforced plastic composites using 'hybridtoughened' matrices, J. Mater. Sci. 41 (2006) 5043-5046.

[29] N. Gardner, E. Wang, A. Shukla, Performance of functionally graded sandwich beams under shock wave loading, Compos. Struct. (2011) DOI 10.1016/j.compstruct.2011.12.006.

[30] E. Wang, N. Gardner, A. Shukla, The blast resistance of sandwich composites with stepwise graded cores, Int. J. Sol. Struct. 46 (2009) 3492-3502.

[31] N. Gardner, E. Wang, P. Kumar, A. Shukla, Blast mitigation in a sandwich composite using graded core with polyurea interlayer, Exp. Mech. (2011) DOI 10.1007/s11340-011-9517-9.

[32] Derakane 8084 Material Data Sheet, www.ashland.com (Accessed September 20, 2011).

[33] Derakane 411-200 Material Data Sheet, www.ashland.com (Accessed September 20, 2011).

[34] Kaneka Kane Ace MX 153 Material Data Sheet, http://www.kanekatexas.com (Accessed September 20, 2011). 
[35] Corecell ${ }^{\mathrm{TM}}$ A-Series Foam Material Data Sheet, http://www.gurit.com/corecellafoam.aspx. (Accessed September 20, 2011).

[36] W. Chen, B. Zhang, M.J. Forrestal, A split Hopkinson bar technique for low impedance materials, Exp. Mech. 39 (1998) 81-85.

[37] P. Kumar, J. LeBlanc, D. Stargel, A. Shukla, Effect of plate curvature on blast response of aluminum panels, Imp. Eng. (2012) Accepted.

[38] J. LeBlanc, A. Shukla, C. Rousseau, A. Bogdanovich, Shock loading of threedimensional woven composite materials, Compos. Struct. 79 (2007) 344- 355.

[39] Wright J. Shock tubes. New York: John Wiley and Sons Inc, 1961.

[40] V. Tiwari, M.A. Sutton, S.R. McNeill, Assessment of High-speed Imaging Systems for 2D and 3D Deformation Measurements: Methodology Development and Validation, Exp. Mech. 47 (2007) 561-579.

[41] V. Tiwari, M.A. Sutton, S.R. McNeill, S. Xu, X. Deng, W.L. Fourney, D. Bretall, Application of 3D Image Correlation for Full-Field Transient Plate Deformation Measurements During Blast Loading, Int. J. Imp. Eng. 36 (2009) 862-874.

[42] H.T. Wu, G.S. Springer, Impact induced stresses, strains, and delaminations caused by impact on composite plates, J. Compos. Mater. 22 (2008) 533-560.

[43] L.J. Gibson, M.F. Ashby. Cellular Solids: Structures and Properties. New York: Pergamon Press, Inc., 1988.

[44] E. Wang, Blast resistance and energy mitigation behavior of sandwich composite materials, Ph.D Dissertation, University of Rhode Island, 2010. 


\section{CHAPTER 7}

\section{CONCLUSIONS AND FUTURE WORKS}

\section{Conclusions}

The main objective of this investigation has been to investigate the blast resistance and mitigation behaviors of novel composites and sandwich structures. Various composite panels and sandwich structures were designed and fabricated with an overall aim to create an optimal structure to withstand high-intensity air-blast loadings. Different materials, ranging from facesheet materials made of E-glass VinylEster (EVE) and nano-scale core-shell rubber (CSR) toughened particles, to core materials including functionally graded styrene acrylonitrile foams (SAN) and Dragonshield polyurea were designed and fabricated. Using the shock tube facility, an air-blast loading, equivalent to those experienced and generated during real-life explosions was applied to the various composites and sandwich structures. 3D- Digital Image Correlation (3D DIC) technique coupled with high-speed imaging was used to obtain the back face out-of-plane deflections and velocities, as well as the in-plane strains during the experiments. Understanding the overall behaviors and failure mechanisms will lead to optimally designed light-weight structures that can mitigate energy and maintain structural integrity when subjected to blast loadings. Due to the increased threat of damage to civilian and defense structures in the form of terrorist attacks and blast loading, a comprehensive understanding on blast mitigation of composites and sandwich structures, as well as an optimal design to withstand these loadings, is pivotal. The findings from the present study are summarized below. 
(1) The dynamic stress-strain response is significantly higher than the quasi-static response for every type of Corecell ${ }^{\mathrm{TM}}$ A-series foam studied, as well as the Dragonshield-HT Polyurea (PU) interlayer. Both quasi-static and dynamic constitutive behaviors of Corecell ${ }^{\mathrm{TM}}$ A-series foams (A300, A400, A500, and A800), as well as the PU interlayer show an increasing trend. The improvement of the mechanical behavior from quasi-static to high strain-rates in these core materials, as well as their long stress plateaus, signifies their ability to absorb large amounts of energy under high strain-rate dynamic loading. Therefore, they show great potential in being used as core materials in sandwich structures subjected to high intensity air blasts.

(2) The sandwich specimens with two different core arrangements, configuration 1 (A300/A500/A800) and configuration 2 (A500/A300/A800), were subjected to shock wave loading. The overall specimen dimensions and areal density were held constant; the only difference was in the gradation of the foam core layers. Configuration 1 was monotonically graded based on increasing the density of the core layers from low/middle/high density, while configuration 2 was nonmonotonically graded, i.e. middle/low/high density foam. The overall performance of configuration 1 (A300/A500/A800) was better than that of configuration 2 (A500/A300/A800). Large compression was visible in the core when the least density foam (A300) is first in contact with the blast loading. This configuration reduced the dynamic pressures seen on the back facesheet, and thus limited the total amount of damage imparted on the specimen. When using the A500 foam first in contact with the blast loading, 
the overall deformation process of the sample was completely different. Compression in the core was limited, and thus the specimen showed a heavy amount of damage.

(3) Sandwich composites with four different core layer arrangements, one, two, three and four layers respectively, were subjected to shock wave loading. The foam core was monotonically graded based on increasing acoustic wave impedance (increasing density and stiffness, E and $\rho$ respectively), with the foam core layer of lowest wave impedance facing the blast. The specimen dimensions were held constant for all core configurations, while the number of core layers varied. The overall performance of the sandwich composite with four layers of core gradation is the best, followed by the sandwich composites with three, two and one layer gradation respectively. Even though each configuration allowed for a stepwise compression of the core, it was shown that the number of core layers has an influence on the dynamic response of the structure under blast loading. More specifically, by increasing the number of monotonically graded layers, the acoustic wave impedance mismatch between successive layers is reduced. Therefore, the strength of the initial shock wave (stress wave) can be weakened by the time it reaches the back facesheet, resulting in lower back face deflection, in-plane strain, and velocity. More importantly, the overall damage imparted on the structure can be reduced and structural integrity can be maintained.

(4) Increasing the number of monotonically graded foam core layers, thus introducing more material interfaces, allows for blast wave (stress wave) 
attenuation through the following mechanisms: (1) stepwise compression of the core (energy dissipation mechanism) and (2) scattering/dispersion of the wave through interface variations. Combining these mechanisms results in lengthened timescales for pressure rises across the samples, allowing for a time-delay of the peak stress arrival, and thus delaying the time of damage initiation.

(5) When using higher levels of core gradation, i.e. two, three and four layers respectively, the amount of stress transferred to subsequent layers is diminished, thereby subjecting the back face to reduced loadings and blast pressures.

(6) Sandwich composites with two types of core layer arrangements were subjected to shock wave loading. Both core configurations consisted of three (3) types of Corecell ${ }^{\mathrm{TM}}$ foam and a polyurea (Dragonshield - HT) interlayer. The foam core was monotonically graded based on increasing wave impedance and the only difference between the two core configurations arose in the location of the polyurea interlayer. It was observed that when the polyurea interlayer is located behind the graded foam core, and in front of the back face (i.e. configuration 2), the core layer arrangement allows for a stepwise compression of the core. Larger compression was visible in the A300 and A500 foam core layers of configuration 2 than configuration 1 . This compression lowers the strength of the initial shock wave by the time it reaches the back facesheet and thus the overall deflection, in-plane strain, and velocity were reduced in comparison to the sandwich composite with the polyurea 
interlayer located behind the front facesheet and in front of the foam core (i.e. configuration 1). Therefore, it can be concluded that placing the polyurea interlayer behind the foam core and in front of the back facesheet (configuration 2) improves the blast resistance of the sandwich composite and better maintains structural integrity.

(7) Comparison of the mid-point deflection of both configurations was made using high-speed photography (side-view images) and the Digital Image Correlation (DIC) technique. Results obtained using both methods of analysis showed excellent agreement with a small margin of error $(<5 \%)$.

(8) The methods used to evaluate the energy as described by Wang et al. (2010) were implemented and the results analyzed. It was observed that the location of the polyurea layer has a significant positive effect on the response of composite sandwich panels to shock wave loading, both in terms of failure mitigation and energy absorption, if it is placed opposite the blast-receiving side (configuration 2). On the contrary, the presence of polyurea on the blastreceiving side (configuration 1), amplifies the destructive effect of the blast, promoting (rather than mitigating) the failure of the composite sandwich panels.

(9) Sandwich composites with two types of monotonically graded cores based on increasing wave impedance were subjected to blast loading. In order to reduce areal density, a sandwich composite with equivalent core layer mass was fabricated and its blast performance was compared to its sandwich composite counterpart with equivalent core layer thickness. The materials, as well as the 
core layer arrangements, and overall specimen dimensions were identical, with the only difference appearing in the thickness of the individual core layers of each specimen (equivalent layer thickness and equivalent layer mass). Table 1 lists the overall results of this specific investigation.

Table 1. Overall comparison of final results

\begin{tabular}{|c|c|c|c|c|}
\hline & Units & $\begin{array}{c}\text { Equivalent } \\
\text { Thickness }\end{array}$ & $\begin{array}{c}\text { Equivalent } \\
\text { Mass }\end{array}$ & Difference \\
\hline Areal Density & $\mathrm{Kg} / \mathrm{m}^{2}$ & 18.5 & 17.6 & $\downarrow \mathbf{5 \%}$ \\
Deflection & $\mathrm{mm}$ & 33 & 42 & $\uparrow \mathbf{2 1 \%}$ \\
In-plane Strain & $\%$ & 2.26 & 2.46 & $\uparrow \mathbf{8 \%}$ \\
Out-of-plane Velocity & $\mathrm{m} / \mathrm{s}$ & 31 & 34 & $\uparrow \mathbf{8 \%}$ \\
Total Energy Loss & $\mathrm{KJ}$ & 7.7 & 9.2 & $\uparrow \mathbf{1 6 \%}$ \\
Deformation Energy & $\mathrm{J}$ & 447.9 & 556.6 & $\uparrow \mathbf{2 0 \%}$ \\
$($ EI) & $\mathrm{N} \mathrm{x} \mathrm{m}{ }^{2}$ & 6.5 & 5.3 & $\downarrow \mathbf{1 8 \%}$ \\
\hline
\end{tabular}

(10) The flexural stiffness and shear rigidity were numerically investigated to achieve a better understanding on the overall behavior of the two types of sandwich composites. It was observed that utilizing a sandwich composite with equivalent core layer mass, thus increasing the thickness of the A300 (softer) layer, decreasing the thickness of the A800 (stiffer) layer and reducing the overall areal density, results in specimen whose stiffness and strength is significantly lower $(\sim 20 \%)$ in comparison to the sandwich specimen with equivalent core layer thickness. 
(11) Core-shell rubber particles have a significant effect on the quasi-static and dynamic behavior of composite materials and sandwich structures. It was observed that the addition of $4 \%$ CSR to E-glass Vinyl-Ester (EVE) composite facesheets increased the tensile strength $(\sim 10 \%)$ and drop-weight impact resistance ( 10\%),. After the dynamic loadings, such as SHPB and shock wave loading, the CSR toughened facesheets maintained structural integrity, while the Non-CSR facesheets did not.

(12) Two types of sandwich composites were fabricated, one with the addition of nano-scale core-shell rubber (CSR) particles during infusion, and one without (Non-CSR). The core material and thickness, as well as overall specimen dimensions were held constant. Results indicated that the addition of nanoscale core-shell rubber (CSR) particles to the panels allows for an increase in blast performance. The improvement in impact resistance, as well as the increased tensile strength of the CSR toughened facesheets, can be directly applied to the increased performance of the CSR toughened sandwich composites. The improvement in impact resistances allows for better dispersion of the initial shock loading, while the increased tensile strength improves the bending strength of the sandwich composites. This results in lower amounts of overall out-of-plane deflection and velocity, as well as inplane strain, approximately $8 \%, 12 \%$ and $20 \%$ respectively in comparison to the Non-CSR toughened sandwich composites. 


\section{Future Work}

The current research is a step forward in understanding the dynamic response of composites panels and sandwich structures during shock wave loading. It elucidates a more comprehensive understanding on the dynamic behavior and mechanisms of failure of novel composite facesheets and sandwich structure subjected to highintensity air-blasts. Different materials, ranging from facesheet materials made of Eglass Vinyl-Ester (EVE) and nano-scale core-shell rubber (CSR) toughened particles, to core materials including functionally graded styrene acrylonitrile foams (SAN) and Dragonshield Polyurea were designed and fabricated. The overall aim was to design and fabricate, through composite toughening and functionally graded foam cores, light-weight structures that can mitigate energy and maintain structural integrity when subjected to blast loadings. The proposed future projects are as follows,

(1) Perform Split Hopkinson Pressure Bar (SHPB) experiments on the foam core of the functionally graded sandwich beams, with a focus on the behavior (amplitude and length) of the reflected and transmitted pulses. By using the identical core layer arrangements, along with the various material interfaces, better insight can be made on the blast performance and behavior of sandwich structures with functionally graded/layered core arrangements.

(2) Conduct a comprehensive study to understand the effect of nano-scale coreshell (CSR) rubber particle weight percentages on the quasi-static and dynamic behaviors of glass-fiber reinforced panels. Due to the fact that CSR particles cause an increase in resin viscosity, the weight percentages of CSR cannot exceed $10 \%$ without compromising proper infusion. Specimens will be 
fabricated and subjected to various quasi-static and dynamic loading conditions including, drop-weight impact, Split Hopkinson Pressure Bar (SHPB) and shock tube loading. Also residual strength measurements of the panels will be conducted both pre-and post-loading.

(3) Results from the composite panel tests will aid in the optimal design of a sandwich structure to mitigate energy and maintain structural integrity. Depending upon the weight-percentage of CSR that provides the best increase in mechanical properties, sandwich panels will be fabricated using the same amount of CSR in the resin system. A shock tube apparatus will be utilized to conduct a controllable and repeatable air-blast loading on the sandwich structure. During the shock tube testing, a high-speed photography system coupled with the optical technique of 3-D Digital Image Correlation (DIC) will be utilized to capture the real-time deformation process as well as mechanisms of failure. Post-mortem analysis will be carried out to evaluate the overall blast performance of this structure.

(4) The idea and application of polyurea, or other rubber-elastomers, to sandwich composites and various other structural materials should be extensively investigated. The aim of the investigation should be focused on the influence of polyurea coating thickness on the dynamic response of the material system. There exists an optimal thickness, depending upon application, which allows for the highest mechanical property increase, without compromising the overall areal density/weight. 
(5) Also the idea of a functionally graded polyurea system could have a significant effect on retrofitting pre- existing structures, and creating more improved blast resistant structures. Using cenospheres or fly ash to increase the density of the polyurea coating would allow for a functionally graded material system based on increasing density. Since polyurea has the ability to be spray cast, functionally grading the polyurea and applying it to structures shows great promise.

(6) An in-depth, cost-effective analysis is needed between the weight and performance of the entire series of sandwich structures investigated. Failure mode maps should be generated for the different core material and facesheet systems used. Focus should be on the failure mechanisms observed and the overall behavior of the sandwich structures, as well as the total amount of deflection, strain and velocity each panel exhibited. 


\section{APPENDICES}

\section{APPENDIX A}

\section{FABRICATION OF SPECIMENS:}

\section{VACCUUM ASSISTED RESIN TRANSFER MOLDING (VARTM) PROCESS}

Composites are defined as a material composed of two or more distinctive constituents. There is a reinforcement phase, which are the fibers, and a binder phase, which is the compliance matrix or resin. Composites offer a range of properties which are impossible to match with traditional materials. They allow the design and engineering of materials precisely tailored to meet the demands of the particular application. Composites combine tremendous durability and high specific strength with an ability to be formed easily and accurately into virtually and complex geometric shape. Some advantages of composites include high strength and stiffness, low weight-mass ratio, dimensional stability and exceptional formability.

When these composites are made in the most economical way, they are inferior to those that take much more time and money to construct. Developing a method that is fast and reliable is crucial. A controlled set up must be designed in order to optimize the resin infusion. The experimental process in which infusion is accomplished is known as Vacuum Assisted Resin Transfer Molding (VARTM). In this process a vacuum pulls resin in from a feed tube to distribute it evenly into the preform.

There are several different steps that must be followed in order to run a VARTM infusion. A selection of materials that will be infused must be acquired. For example, sixteen (16) plies of E-glass are cut and stacked in a quasi-isotropic layout $\left[0^{\circ} / 45^{\circ} / 90^{\circ} /-45^{\circ}\right]_{\mathrm{s}}$. There are eight (8) plies on the bottom, and eight (8) plies on the top, with a foam core separating the E-glass fibers. This creates a sandwich composite panel and is the preform that will be infused (Figure 1a). The foam core has been previously laid out in a configuration that was tailored to meet specific demands. Once the core was laid out, 3/16 inch holes were drilled approximately two inches apart and off center (Figure 1b). This ensures that the resin will flow through the entire preform and infuse properly. 


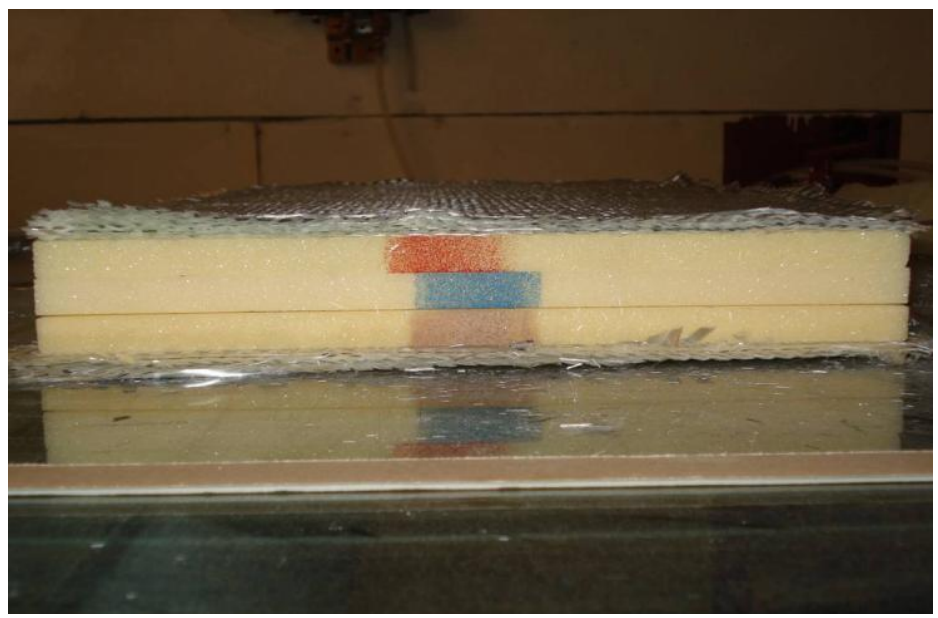

(a) Preform layout (E-Glass + Foam Core)

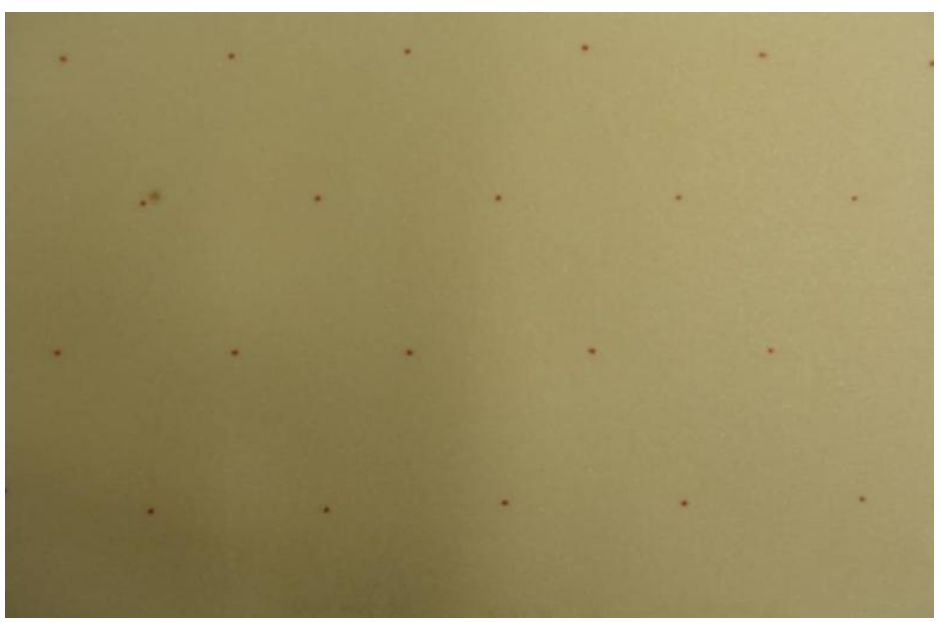

(b) Layout for drilling holes in foam core

Fig. 1 Sandwich composite perform layup

The preform is placed on a large glass table that has already been coated with a few layers of high-temperature Mold Release (T.R. Industries). This is a substance that deters two substances from bonding together, also known as a buffer. This is important so that the finished panel does not stick to the glass and can easily be removed. The next step is to place a sheet of peel ply on top of the preform, so the vacuum bag does not stick to the finished composite panel (Figure 2a). The peel ply is a highly permeable fabric allowing resin to flow through it, but not hardening with the preform. When the process is finished, the peel ply allows the preform to have uniform texture.

After the peel ply is laid down, a piece of mesh is cut to approximately the dimensions as the top of the preform ( $\sim 1$ in. on all sides), and placed on top of the peel 
ply. This mesh helps ensure proper infusion across the perform (Figure 2b). Next, a distribution medium (knitted fabric) is placed alongside the performs to ensure that thorough wetting out is achieved quickly. This entire process is known as SCRIMP (Seemann Composite Resin Infusion Molding Process)

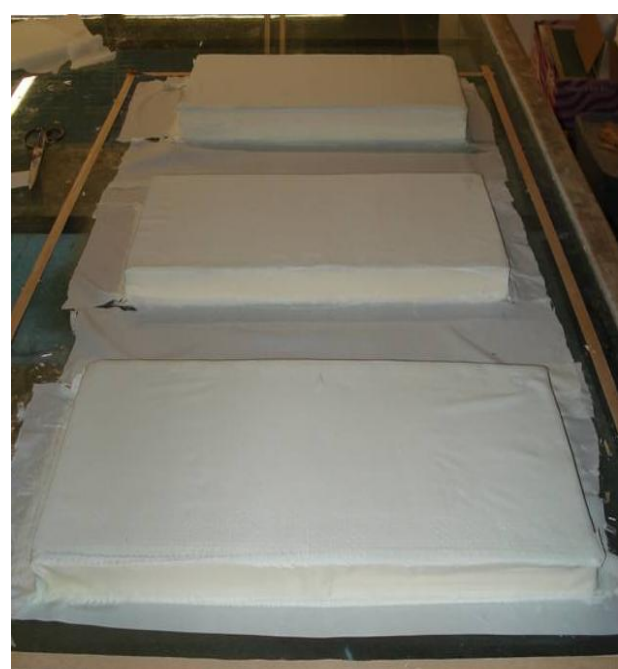

(a) Peel ply laid over preform

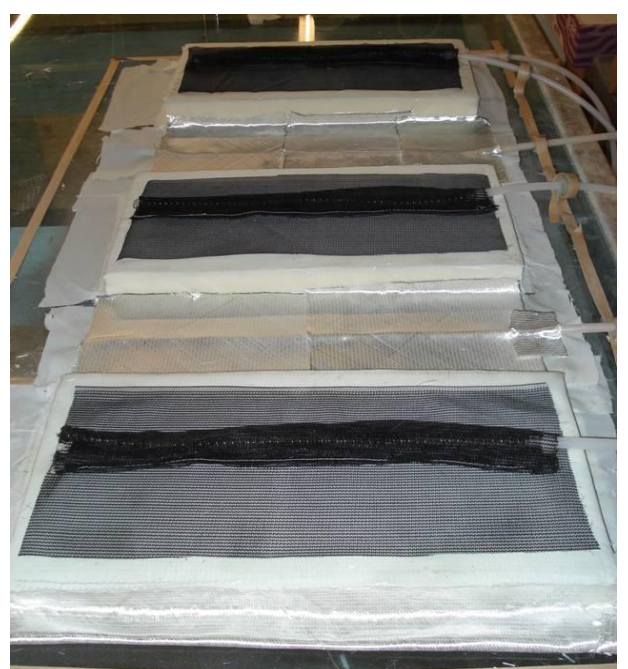

(b) Mesh placed on top of peel ply

Fig. 2 Seemann Composite Resin Infusion Molding Process (SCRIMP)

Tacky tape forms the perimeter around the entire preform. It is placed about two (2) to three (3) inches wider than the preform to give adequate room for tubing and bagging. A piece of coiled tubing, which has been cut to the approximate length of the preform, is placed on the sides of the preform. Another piece of tubing, which is connected to the vacuum pot, is inserted into the coiled tubing. The vacuum pot is a sealed bucket that collects any excess resin that comes out of the preform. This is important because the vacuum pump would be ruined if resin enters the vacuum line. A feed line is then installed on top of the preform. Again, a piece of coiled tubing is cut to the approximate length of the preform. Once the coiled tubing is in place, it is wrapped with mesh and a piece of tubing, which is connected to the resin bucket, is inserted into the coiled tubing. These lines are made airtight by wrapping tacky tape around the edges that cross the previously constructed tacky tape perimeter (Figure 5).

The final step in the set-up is the addition of the vacuum bag. The bag is adhered to the tacky tape and positioned around the tubes (Figure 6). When there is excess bag in an area, an "ear" is formed with tacky tape to guarantee that the bag will be airtight. 
This is a critical step due to the fact that any small holes could cause loss of a full vacuum.

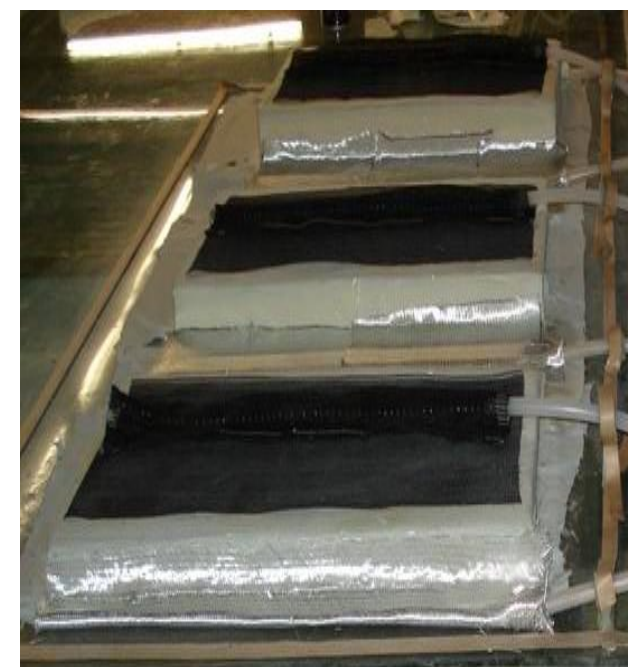

(a) Tacky tape with resin and vacuum tubes

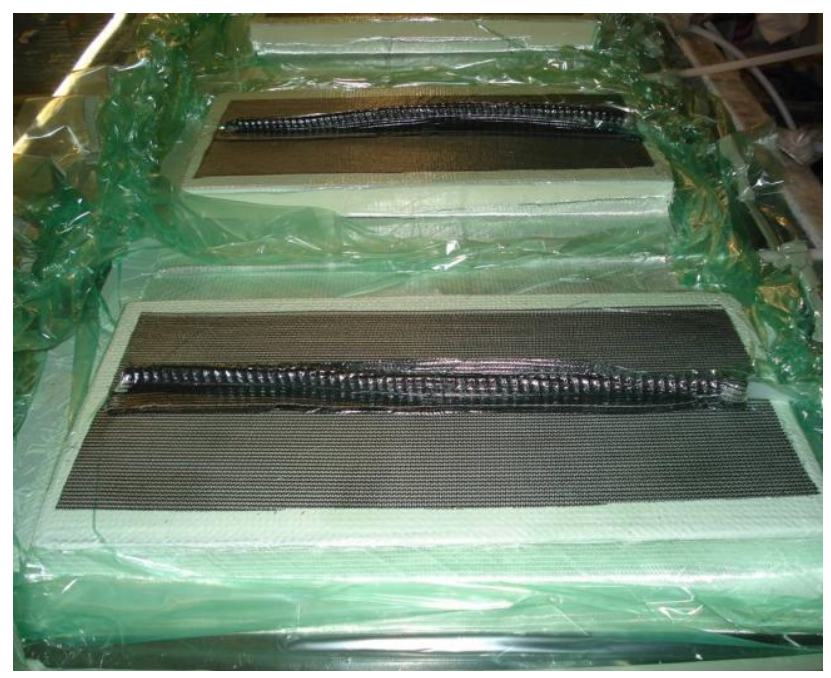

(b) Vacuum bag attached and sealed

Fig. 3 Preparation for Vacuum Bag

Once the entire set-up is complete, a vacuum check is made. This is done by using a vacuum gage to test how well it was holding a vacuum. After the vacuum check is made, and an adequate vacuum is reached, the clamp is turned off and the infusion begins (Fig. 4).

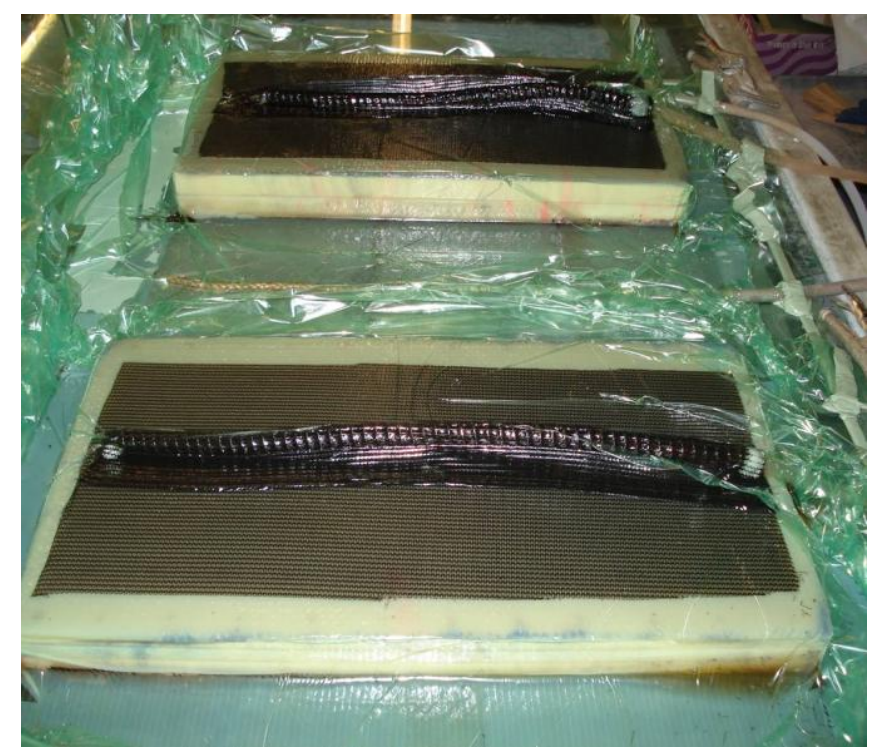

Fig. 4 Preform being infused 
Numerous factors in the set-up process can modify the desired final product. A correct bagging job is one that is not too tight and has no leaks or creases. A bag that is too tight could leave channels running on the sides of the preform, causing the resin to move around the piece on the sides rather than through the preform. This occurs because fluids travel through the path of least resistance. Most of the resin will flow in these voids and leave through the vacuum tube. Therefore, only some resin will enter the piece leaving dry spots and thus weakening the composite.

Another variable is the time needed to perform an infusion. There were a total of sixteen plies of E-glass and a foam core infused at once. The time needed depends on the resin used and the temperature and humidity in the room where the infusion took place. Not enough humidity and/or too low of a temperature can cause the resin to become more viscous. This will lead to improper infusion due to the fact that the resin will harden before the entire sample is infused.

Atmospheric pressure plays another role in the VARTM process. There is pressure pushing against the vacuum bag and pressure pushing against the bucket of resin. If the pressure is higher, the vacuum on the piece will be much higher. The atmospheric pressure pushes on the vacuum bag, causing more air to be withdrawn by the vacuum pump.

Maintaining a perfect vacuum is very important as well. An ideal vacuum is 30" Hg. There are many places where leaks could exist. The vacuum pot must be tightly shut so that no air can leak in from the sides of the lid. In some cases, air that is between the clamp on the feed tube and the resin bucket will get into the piece as the resin follows. This air makes the piece fluctuate rapidly during the entire infusion.

Placing the vacuum and feed tubes in the correct locations can determine how quickly and effectively the resin flows into the preform. Feed tubes should not exceed the length of the preform. The tube should go on top of the preform and in the center. This will prevent dry spots from occurring by forcing the resin to flow through the piece instead of down the sides.

If the coil tubing on top of the preform became loose, it could puncture the vacuum bag. This would hinder the quality of the vacuum, making the infusion process take longer and weaken the compression that the bag puts on the piece. Also, 
if the tacky tape does not stay sealed to the glass table, it can create holes in which air will leak in. This could destroy the vacuum and any chance for a quality piece to form.

When the infusion process is completed, the piece must be left to cure. This is the process in which the resin will harden. There are a couple of ways in which the piece can cure. It can either be left at room temperature for approximately forty-eight (48) hours, or, to speed up the process, it can be placed in an oven. Leaving the piece on the table, and allowing it to cure, is the best method because it will ensure that the piece remains flat and no bowing occurs. Also, if the temperature of the oven is set too high, the sides of the piece will flare up and the entire piece will become less dense. This will lead to an offset of the strength properties of the piece.

\section{References}

[1] Cook J and Walsh S (2003) Enhancing the Vacuum Assisted Resin Transfer Molding (VARTM) process through the alternation of variables. Army Research Laboratory.

\section{Extra Figures:}

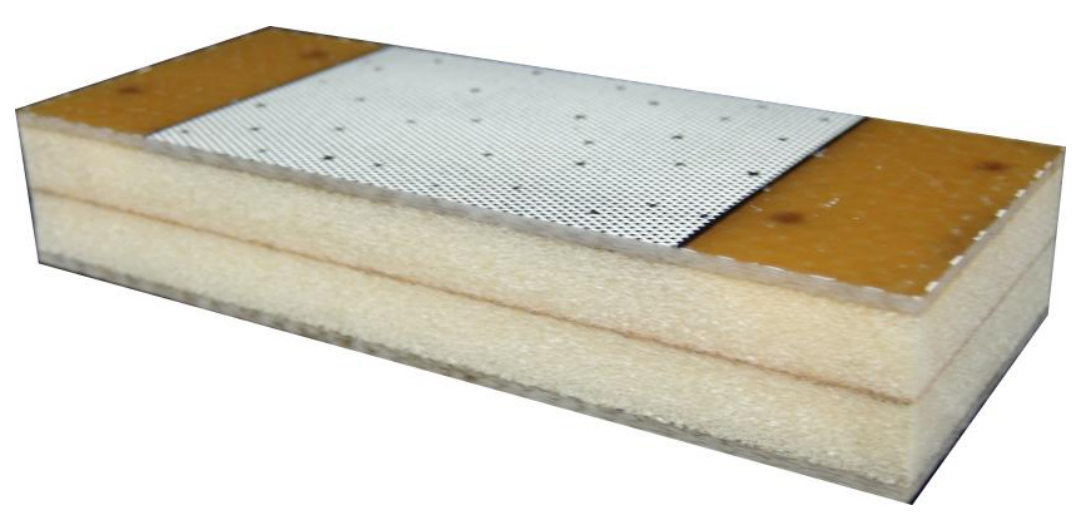

Fig. 5 One Layer Gradation

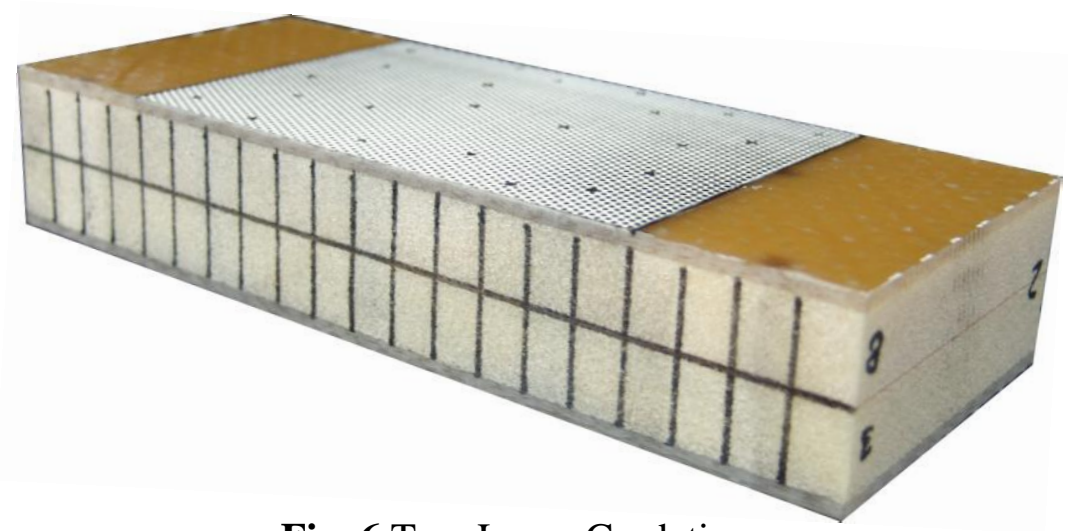

Fig. 6 Two Layer Gradation 


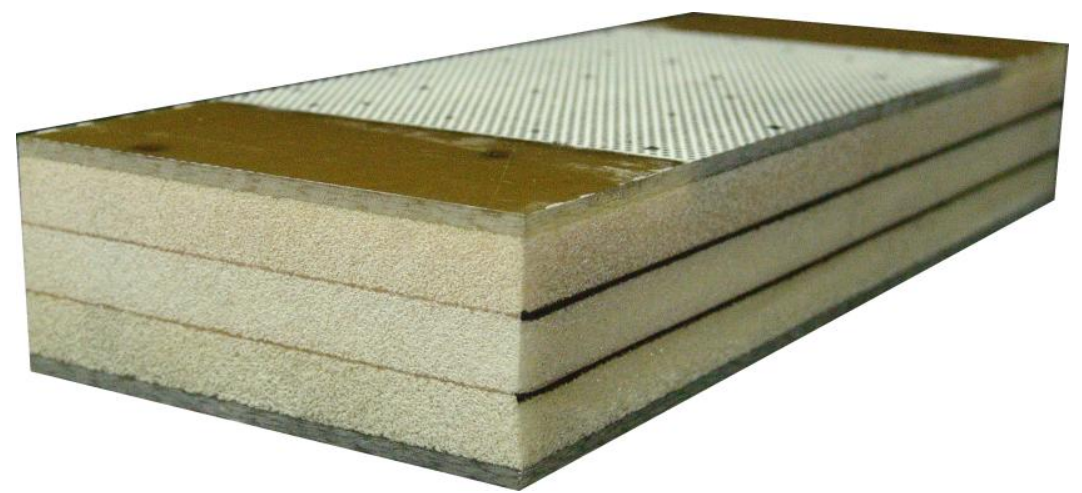

Fig. 7 Three Layer Gradation

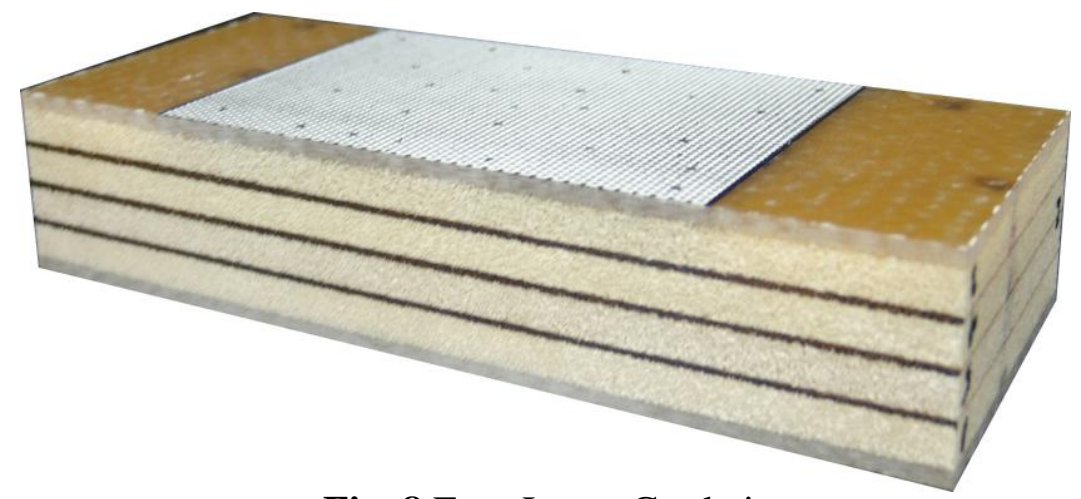

Fig. 8 Four Layer Gradation

\section{POLYUREA FABRICATION:}

(1) Infuse the foam core and one-8ply facesheet

(2) Infuse second 8ply facesheet

(3) Cut fabricated panels to overall specified dimensions ( $304.8 \mathrm{~mm}$ x $609.6 \mathrm{~mm}$ )

(4) Plasma treat both sides of the Dragonshield-HT polyurea layer

(5) Sand the surface of the facesheet and foam core for which the polyurea interlayer will be in contact with

(6) Mix West Systems Epoxy 105/206 and apply to both sides of the polyurea interlayer

(7) Place sandwich panel under vacuum again to ensure uniform pressure over entire sample 


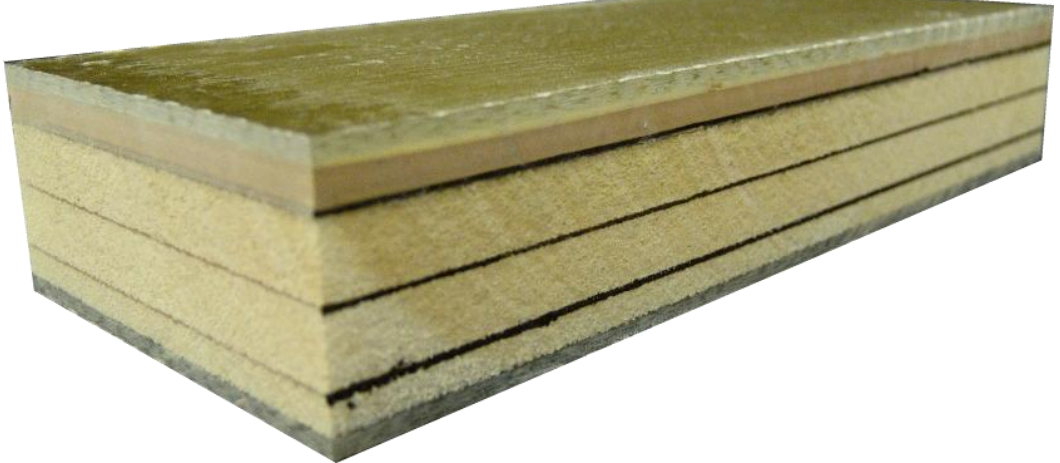

Fig. 9 PU Front (PU/A300/A500/A800)

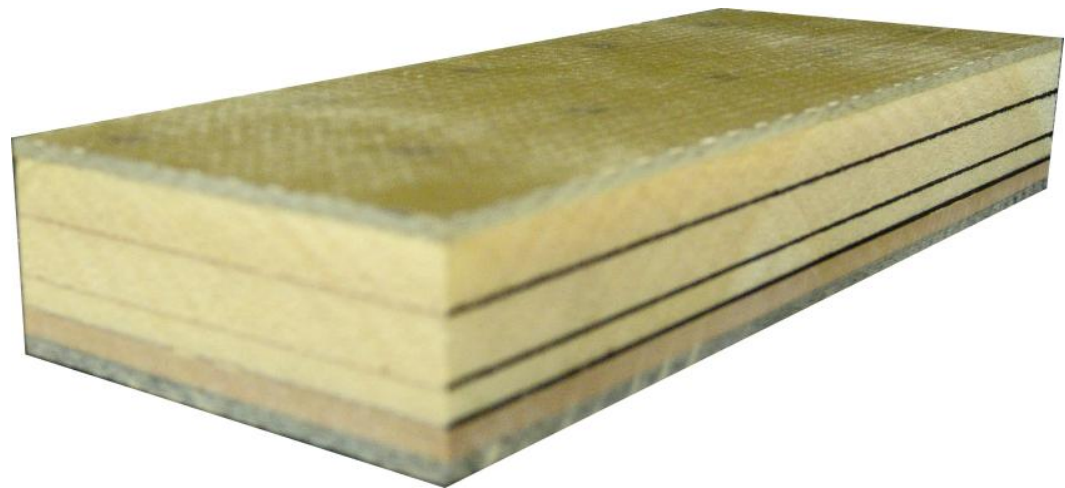

Fig. 10 PU Back (A300/A500/A800/PU) 


\section{APPENDIX B}

\section{STANDARD OPERATING PROCEDURES (SOP)}

\section{$\underline{\text { SHPB }}$}

\section{Specimen preparation:}

1. The specimen dimensions should follow the below relation:

$$
\frac{L}{D}=\sqrt{\frac{3 v}{4}}
$$

where $\mathrm{L}$ is the length, $\mathrm{D}$ is the diameter and $v$ is the Poisson's ratio of the specimen.

2. Make sure the faces of the specimen are parallel and flat (use step collet while machining metal specimens to get parallel faces). The diameter of the specimen after the test should be smaller than the diameter of the pressure bars.

\section{Selecting the bar:}

1. Determine the impedance ( $\rho c A)$ of the specimen.

where $\rho$ is the density, $c\left(c=\sqrt{\frac{E}{\rho}}\right)$ is the wave speed and $A$ is the area.

2. Then select the pressure bars (steel or Aluminum) closer to the impedance of the specimen. We also have different diameters for the pressure bars.

Note: The basic thumb rule is that we use steel bars for the harder materials (metals etc..) and Aluminum bars for the softer materials (polymers, foams etc..).

3. After the pressure bars are selected, make sure the end faces of the bars are flat and parallel.

4. Align the pressure bars and striker on the mounting frame.

\section{Experimental procedure:}

(a) Give all required connections. Connections include: Connect the BNC cables from the amplifier to the oscilloscope. Check the right channels and connect them. Make sure the amplifier (2310A) and oscilloscope are grounded. Do not change any settings on the amplifier. The amplifier has been calibrated for $350 \mathrm{ohms}$ strain gages. Please refer to manual if you we 
wish to make any changes and let everyone in the lab know before you make any changes. Turn ON the amplifier and oscilloscope.

(a) The excitation voltage and gain are set to $10 \mathrm{~V}$ and 100 respectively. Turn the reset switch ON for all the four channels.

(b) Check the resistance on the strain gauges and they should read around 350 ohms.

(c) Set the voltage levels, trigger position, data duration time (2ms-4ms), for all the four channels in Oscilloscope. These values depend on the experiments.

(d) Balance the Wheatstone bridge for all the four channels by turning the reset button.

(e) Check whether the bars are well aligned or not, and also the projectile should be well aligned to the impact end of the incident bar.

(f) Then make sure that the bars are moving freely, if not apply WD-40 lubricant and adjust the screws of the clamps.

(g) Clean the interfaces of the bar and the projectile with Kim wipes and ethyl alcohol.

(h) Push the projectile to the end of the barrel of gas gun assembly with a flexible poly rod.

(i) Measure the dimensions of both specimen and pulse shaper. Dimensions include: diameter and thickness.

(j) Select the striker depending on the strain rate you are trying to get. You can vary strain rate by using different pressures and different striker bars. Make sure the pulses are not getting overlapped. If the pulses are getting overlapped, use the shorter striker bar. (Thumb rule: The longer the striker, the lower the strain rate. The higher the pressure, the higher the strain rate).

(k) Lubricate both faces of the test specimen with Molybdenum disulfide lubricant and sandwich the specimen between the bars and align the specimen with respect to bar center.

(1) Place the pulse shaper at the impact end of the incident bar with a thin layer of lubrication (if you are using lead pulse shaper) and align it with respect 
to bar center. We generally use clay and lead pulse shapers. These give us very good results for harder materials, but for the softer materials, you can try different pulse shapers. These include paper, copper etc..

(m) Release the nitrogen gas from the gas tank into the gas gun chamber until the required pressure level is achieved.

(n) Arm the oscillation to capture the strain gage voltage signals and make sure the arm holds until you release the projectile. If the arm is not holding, adjust trigger levels. (Note: if you are getting high noise in your signals more than $20 \mathrm{mv}$, turn off the lights before the experiment).

(o) Once again, ensure that the specimen is well aligned between the bars and verify the status of the trigger hold before pressing the solenoid valve release button.

(p) Press solenoid valve control box button to release the projectile.

(q) Save captured voltage pulses onto a USB drive for further analysis of the data.

(r) A MATLAB program is written to read the data from the pulses and analyze the pulses using the one-dimensional wave theory stress and strain equations. After the experiments are performed, the pulses are used along with the MATLAB program to determine the equilibrium and true stressstrain plots of the specimen.

(s) After the experiment is completed, turn off the cylinder and make sure all the left over nitrogen gas in the gas chamber is released.

(t) After the data is transferred from the oscilloscope to USB drive, verify that in your computer and turn off the amplifier and oscilloscope.

\section{Analyzing the results:}

1. There are two MATLAB codes to analyze the data. 1. Verify_Equilibrium and 2. Steel/aluminum_SHPB. Use the appropriate codes to analyze the data. Depending on the bars you used, the respective code has to be used.

2. Make sure the code has the right properties and dimensions of the pressure bars you used. These include the bar diameters and proper wave speed of bar material. 
3. If we use hollow bars, make sure you have the right dimensions in the code. For solid bars, dimensions for the hollow bar should be zero.

4. First run the verify equilibrium code. Make sure the data you get from the oscilloscope has the following names for the four channels. TEK00000, TEK00001, TEK00002, and TEK00003. The code recognizes these names. Make sure the codes and the data are in the same folder.

5. TEK00000 and TEK00001 represent incident and reflected pulses (channel 1 and channel2). TEK00002 and TEK00003 represent the transmitted pulse (channel 3 and channel 4). The code averages channel 1 and channel 2. And channel 3 and channel 4 .

6. The code converts the voltage output to microstrains and balances.

7. Default values for filtering are given in the code. For incident and reflected pulse, default value of 0.2 ( $\mathrm{fn}=0.2$ ) is used and for transmitted pulse, a value of 0.05 ( $\mathrm{fn}=0.05$ ) is used. Depending on the noise you get, change the values of fn. The value of 'fn' ranges from 0.001 to 0.99 . Higher value of ' $f n$ ' means, the pulses were not filtered. Decrease the value of ' $\mathrm{fn}$ ' if you would like to filter more. You can use different values for incident and transmitted pulses.

8. When you run the code, you get two figures. Figure 1 gives the incident and reflected pulses. Figure 2 gives the transmitted pulse.
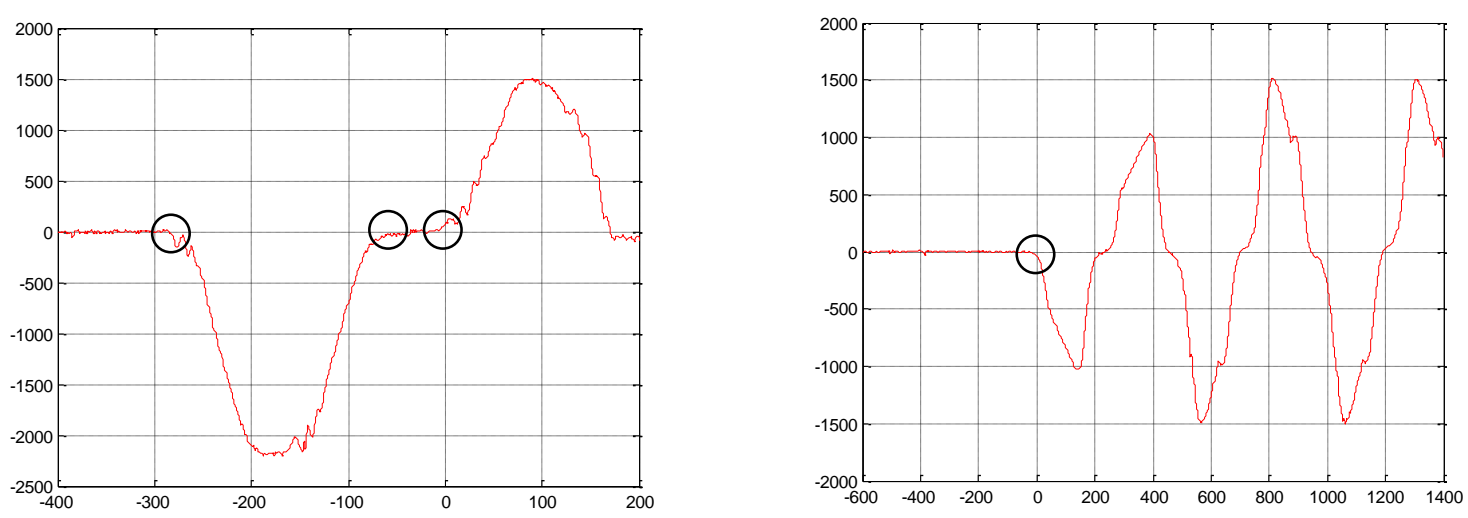

9. Note the incident starting time, incident end time, reflected starting time and transmitted starting time as shown in the above figures. You can zoom the pulses by pressing 'zoom in' button at the top to get the right times. Then go to MATLAB main window and press 'ENTER'. 
10. Input the values you found out and press 'ENTER'.

11. Now you will get 3 more figures. Figure 3 shows the incident, reflected and transmitted pulses. Figure 4 shows the incident, reflected and transmitted pulses you picked on before. Figure 5 shows the force ratio. Front face represents the forces calculated on the incident and reflected pulses. Back face represents the force calculated on the transmitted pulse. Ideally, these two fronts and back face should match perfectly.

12. Various factors decide the equilibrium. These include type of material tested, strain rate etc..

13. Make sure the incident and reflected pulses start at the same time on Figure 4. On the first trial, you might end up something as below

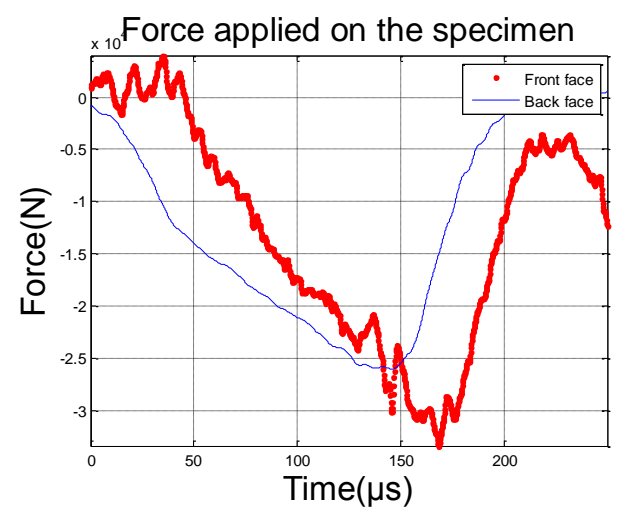

14. Go back to the times you found for the incident, reflected and transmitted pulses. Never change the times of reflected and transmitted pulses. Shift the incident pulse to either side and try for different values until you get decent equilibrium. For the case shown above, by shifting the time of the incident pulse, the below equilibrium was obtained.

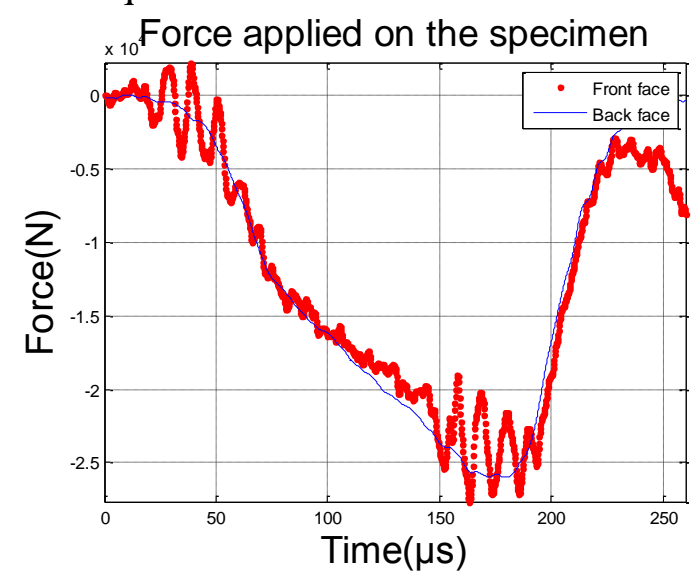


15. Save this figure in to the respective folder. Also save the new times of the pulses.

16. Now open the SHPB code and make sure you have the same value for filter as in the verify_equilibrium code.

17. Enter the specimen thickness and diameter in inches.

18. Again, you get two figures. Figure 1 gives the incident and reflected pulses. Figure 2 gives the transmitted pulse.

19. Go to main 'MATLAB' window and enter the final times here.

20. You get Eng. stress strain curve (Figure 3) and True stress-strain curve (Figure 4).

21. Follow the directions of the Figure 4.

22. Pick two points to calculate the slope. You can pick at the initial elastic region of the true stress-strain curve.

23. You will end up with figure 5. Pick two points at the linear region as shown below.

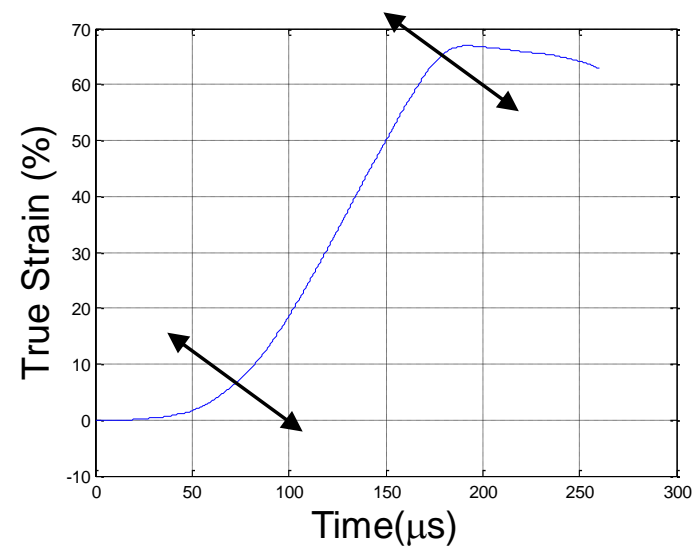

24. Go to MATLAB main window and you can see the strain rate. Note down this value. Next you will end up with final figure (Figure 6). This is eng. strain rate vs. time.

25. Be careful when you pick up the strain rate points. Consider the following points

a. Make sure the region you pick is in the equilibrium. 
b. For foam materials, you might not get very good equilibrium and constant strain rate. So calculate the strain rate over the entire loading duration.

\section{Tensile SHPB:}

\section{Procedure:}

1. The specimen dimensions are given below. These dimensions vary with the material tested. For metals, the below dimensions can be used. To perform experiments at lower strain rate, increase the gage diameter (D) to 0.2 ". For plastics, use gage length of 0.2 " and gage diameter of 0.2 ".

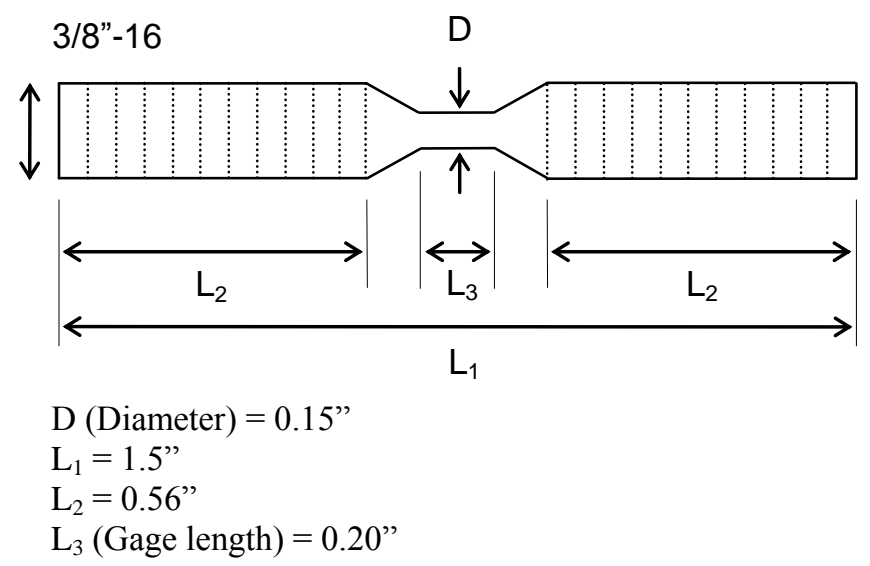

2. Selecting the bar is same as explained before.

3. Experimental procedure is also similar to the above. Here, you place the pulse shaper on the flange. You can use paper, clay or lead.

4. Different striker bars can be used to perform experiments at different strain rates. Make sure the striker bar slides freely on the bars.

5. The specimen will be threaded at both ends to the pressure bars. There is no need to use the lubricant.

6. The connections remain the same as explained before. You can use the same amplifier and oscilloscope, and same settings.

7. The MATLAB codes have been modified and use the appropriate code to perform your analysis. The steps to run the code is same as explained for compression SHPB. 


\section{Compression SHPB at elevated temperatures:}

1. The tungsten carbide inserts will be used. The specimen will be sandwiched between these inserts.

2. The diameter of the specimen should be smaller than the inserts. The below figure shows the set up.

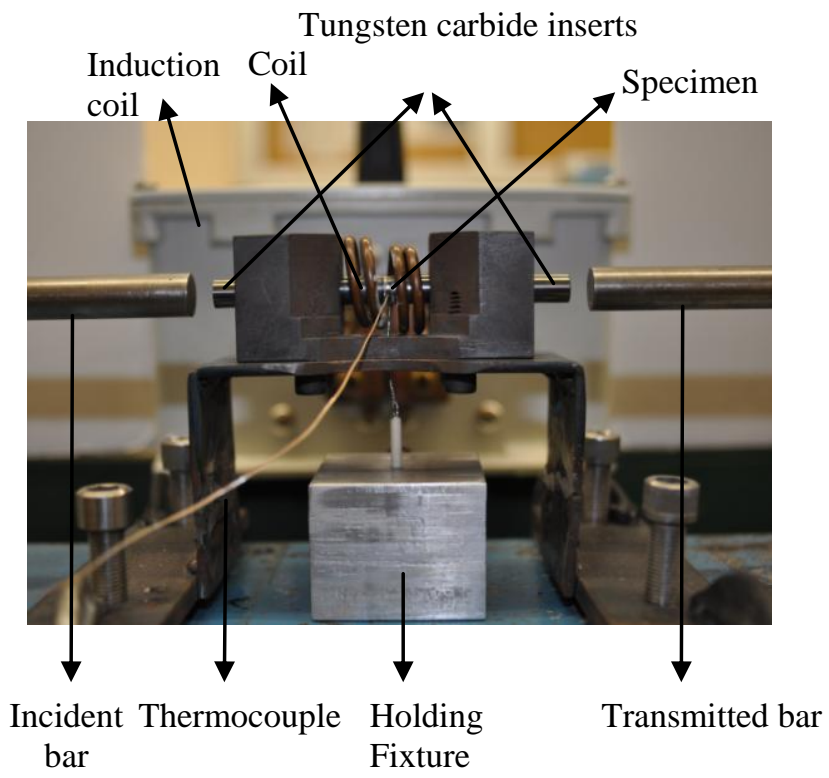

3. For experiments at elevated temperatures, the SHPB apparatus in conjunction with the induction coil heating system will be utilized as shown in Fig. 2.

4. A special fixture is used to load the specimen.

5. The inserts were used to eliminate the temperature gradient in the bars and thus protect the strain gages mounted on them.

6. The impedance of the inserts was matched with the bars; hence they do not disturb the stress wave profiles in the bar. The impedance matching requires the diameter of these tungsten carbide inserts to be smaller than the main pressure bars. This is the reason for the specimen diameter for high temperature testing being smaller than that for room temperature testing.

7. By varying the power, higher temperatures can be achieved.

8. The induction coil heating system has a power control box, remote to start and stop, a cooling unit and cooling supply (blue box) to reserve water. Make sure the blue box has sufficient distilled water. The copper coils are connected to the cooling unit and it is places around the inserts. 
9. First turn $\mathrm{ON}$ the blue box, then the power supply. The power supply needs the larger output in the DPML lab.

10. Make sure the wheel on the cooling unit is pinning smoothly and fast. If not, do not do the experiment. Increase the power supply, to heat the specimen.

11. When the regulator is turned ON, it should give a click sound after around 30s. If it does not, turn it off and try again. If the problem persists, turn off the regulator and the problem can be determined.

12. Turning ON the power supply regulator, it will read 'cycle continuous' on the remote (smallest one), which is desired.

13. The system should already be set to manual power output again, which will allow to control the power. If it is not set, you can do by using the switch located to the immediate right of the dial on the regulator.

14. Make sure the dial on the regulator is zero, so there will be no immediate power output.

15. Now press 'start' button on the remote (small one that reads the display).

16. The bars were kept apart initially, later the specimen and carbide inserts were heated in isolation to the desired temperature (usually about $20-50^{\circ} \mathrm{C}$ higher than the test temperature) and soon after the bars were brought manually into contact with the specimen. The temperature of the specimen was monitored using a $0.127 \mathrm{~mm}$ chromel/alumel thermocouple, which was spot welded onto the specimen.

17. In most of the experiments, it takes less than two minutes to heat the specimen to the required temperature and it takes less than 10 seconds to bring the pressure bars into contact with the tungsten inserts and fire the gun.

18. Once the temperature is reached, hit 'stop' on the display and turn off the regulator and the induction heater. Now trigger the oscilloscope. If you trigger the oscilloscope before, due to magnetic fields from the induction heater, you will see lot of noise.

19. Allow the cooling unit to run for some time son that it reaches room temperature. 
20. All other experimental procedure, data capturing, and analyzing the results remain the same as explained in compression SHPB section.

\section{Note:}

1. Always make sure the yield strength of the material you are testing is never beyond the yield strength pressure bars.

2. For testing ceramics of high strength, we need to use inserts so as to protect the bars from plastic deformation. 


\section{SHOCK TUBE}

\section{Checklist Before Experiments:}

Shock Tube Related:

Do you have enough GAS and GAS PRESSURE?

Do you have enough MYLAR SHEETS?

Dump Tank Related:

Is the support FIXTURE tight in the dump tank?

Are the supports in the right SPAN position?

According to your experiment, do you need the EXTRA SPACERS for the support?

Is the side window, LEXAN SHEET, clean?

Are the back windows, LEXAN SHEETS, clean?

Are the back windows, LEXAN SHEETS, 1/2 in. thick?

Visualization System Related:

Does every LIGHT work fine?

Does every CAMERA work fine?

Does LAPTOP work fine?

Specimen Related:

Have you measured the specimen's SIZE?

Have you measured the specimen's WEIGHT?

Is your SPECKLE PATTERN OK?

Have you taken the pre-blast IMAGES of the specimen? 


\section{Checklist During Experiments:}

\section{Shock Tube Related:}

Have you changed the MYLARS?

Are all the screw connections TIGHT?

Have you put the PRESSURE SENSORS in the shock tube?

Is the shock tube PERPENDICULAR to the supports, and centrally located?

Dump Tank Related:

Is the specimen in the right position and secure?

Is the shock tube close enough to the specimen, $1 / 16$ in?

Have you put the side doors on the dump tank?

If no back side DIC system is required, have you installed a good protection for the back side LEXAN windows ?

\section{DIC System Related:}

Have you double checked the camera CABLE CONNECTIONS?

Is the back view camera system PERPENDICULAR to the back side window?

Is the side view camera PERPENDICULAR to the side window and specimen?

Have you set the right FRAME RATE?

Have you done the BLACK CALIBRATION (SHADING)?

Is the viewing area acceptable (make the view of the specimen as large as it can)?

Have you FOCUSED on the specimen (use iris 2.8 or smaller)?

Have you increased the IRIS to at least 5.6?

Have you set SYNC MODE for all slave cameras (E-SYNC)?

Have you done the correct CALIBRATION (grids used to track particle distance)?

Have you set the right TRIGGER MODE?

Have you double checked that the cameras can take the desired images? 
Oscilloscope Related:

Have you double checked the CABLE CONNECTIONS from the sensors to the channels are right?

Have you double checked the VOLTAGE AND TIME RANGE setting?

Have you double checked the TRIGGER setting (trigger level and position)? 


\section{ULTRAH HIGH-SPEED AND HIGH-SPEED PHOTOGRAPHY SYSTEMS:}

\section{$\underline{\text { IMACON200A }}$}

\section{Set camera facing desired event}

1. Connect control i/o cable, which runs from camera to computer, to camera (should already be attached to computer)

2. Connect a BNC cable from the monitor output of the camera (clearly marked) to the stereo converter box used to connect trigger to photogenic flash light.

3. Connect a BNC cable to trigger 1 (clearly marked) on the camera, and utilizing a $\mathrm{T}$ joint, connect the trigger to the oscilloscope, with a second $\mathrm{BNC}$ branching to the make circuit used to trigger the apparatus.

4. When selecting which Photogenic to use, be sure that one is the flash with the bulb capable of producing persistent light - this will be identifiable as the bulb with an additional, smaller bulb, situated in the center of the flash ring of the rest of the bulb.

\section{FOR CAMERA SETTINGS, put camera into Focus mode and set the} following:
a. Set Iris to $\mathrm{f} 2.8$
b. Set exposure to 5 microseconds
c. Set Gain to 5

6. To focus camera, set flash with the ability to provide a constant light source to model, so it provides light with which to focus the camera. Focus the camera as you would any other camera, by use of the lens manual focus. NOTE: The camera must be in focus mode in order to focus the lens.

7. Once camera, flash, and trigger are set, open the camera software on the computer system.

8. Program provides instruction on setting any additional necessary settings, which can be tailored to your experiment

9. MAKE NOTE:

a. The flash has a rise time of 100 microseconds - be sure that the preflash delay is at least that much 
b. Set MCP to 3 or 4 in computer software - the higher the \#, the higher the brightness

c. The camera delay must also be tailored to your experiment - know how long your event takes, what part of it you wish to photograph, and how long it takes before that happens. The Imacon, though indeed quite mighty, only takes 16 pictures, so you must be sure not to miss your event!

\section{TAKE CAUTION:}

a. When changing the lens, be sure that the shudder of the camera is closed - if it is open, and there is no lens on the camera, ambient light or any sudden flashes can/will destroy the ISOs and the prisms, rendering the camera useless.

b. When testing the flash for operation, do not look directly into it, or flash it while others are doing so - the flash is EXTREMELY intense, and can cause disorientation and result in injury.

11. FINAL NOTE: The Imacon 200 was the first of its kind, and is worth $\$ 500,000$. Treat it with respect - handle it and its components with care 


\section{DIGITAL IMAGE CORRELATION (DIC) ANALYSIS}

\section{TEST AND CAMERA PROCEDURE}

\section{Physical Set-up - Prior to any software}

1. Set up lamps

2. Keep lens caps on cameras

3. Plug in cameras

1. Turn on master

2. Turn on slave

3. Wait for "sync in" light on slave camera before starting any software

4. Make sure IP light is also on

\section{Software Set-up}

1. Start software - PFV

2. Go to Cam2 window

1. Right click

1. CAM options

\section{1. $\mathrm{I} / \mathrm{O}$}

1. Set sync in to "ON CAM POS"

1. E-Sync shows up in window

3. Set frame rate to $20,000 \mathrm{fps}$ (or desired rate)

4. Shading

1. Make sure lens cap is on

1. Click calibrate

2. Do this for each camera!!!

5. Now all set with software set-up

\section{Camera Set-up (Focus and Calibration)}

1. Take lens caps off

2. Focus and set aperture on the lens itself

1. Set aperture to 2.8 initially for focusing the camera 
2. Maximize window of interest in the software for focusing and hit fit

3. Set aperture to 5.6 for calibration

4. Repeat for each camera

3. Calibrate the Cameras

1. Trig Mode $=$ Random, 1 frame

2. Hit Record button in software

3. Move the calibration sheet and hit the trigger button repeatedly while rotating and translating the cal sheet

4. Once sufficient images (>100) are captured hit record done

4. Data Save Tab

1. Select the folder to save the calibration images

1. Save CAM 1 (Cal_0_), Type = TIFF

2. Save CAM 2 (Cal_1_), Type $=$ TIFF

3. Run Through the calibration process in VIC-3D

\section{Camera Usage (Actual Test)}

1. In the camera software

1. Camera Tab

1. Hit Display "Live"

2. Set Trigger mode to "END"

3. Hit record button (software) one time

1. Light goes to orange, and will say Trig In

4. Hit the Trigger (physical) button to begin recording

5. Hit the Trigger button again to stop recording

1. The software will record the images for the 1 second prior to the stop recording push. Therefore, you should stop the recording as soon as possible after the event or you will miss the data.

6. DO NOT TURN OFF THE CAMERAS UNTIL THE DATA HAS BEEN SAVED

7. Check the start point of motion with the red slider bar (the middle number is the current frame) 
1. Hit left facing arrow to set begin point to current frame

2. Play until end of motion and hit stop

3. Hit right arrow to set end of frames

You have now isolated the frames that comprise the event

\section{Data Save}

1. Set the folder for the data to be saved to

1. This is different than the folder the calibration is saved to

2. Select Camera 1

1. File names (Data_0_), Type $=$ TIFF

2. Save

3. Select Camera 2

1. File names (Data_1_), Type $=$ TIFF

2. Save

\section{DATA CALIBRATION AND POST PROCESSING}

1. Open VIC-3-D Program

2. Click Grid Icon (Cal Images)

1. Find Calibration images from test

2. Open all

3. Click caliper button

1. Select Target $(12 \times 12 \times 9$ or whatever used during test)

2. Extract

3. Auto

4. Calibrate

1. Standard Deviation should be $<0.1$ and should have used at least 75 images

5. Now you are done with the calibration part

4. Click the speckle images icon

1. Select the images recorded during the test

2. Under AOI tools tab 
1. Select the type of view area (rectangle, polygon, etc.)

2. Select the view area

3. Take the green box and move to a area of low deformation with a high quality speckle dot

4. Click the "?" icon

1. Dic_0 $\rightarrow$ camera 1

2. Dic_1 $\rightarrow$ camera 2

3. Verify that both cameras are seeing the dot with a checkmark next to the image by scrolling through the images with the down arrow. If there is not a checkmark next to each image then go back and select a new green box location

4. Click close

5. Now all ready for post processing

5. Click the "start analysis" button

1. Run

2. The code will run through the images with Z Displacement initially

3. Done

6. Data drop down menu

1. Post processing

1. Calculate curvature

2. Calculate strain

3. Calculate velocity

1. Input time step as the inter frame time

4. Close

7. Data tab under AOI Tools

1. Can click on the individual pictures now

2. Right click to change contour levels

3. Plot tools $\rightarrow$ Autoscale

1. Uncheck Boxes

4. Contour, set range 
5. Right click to create animations

1. Different coding methods increase or decrease the file size

6. Inspector tools

1. Point, Line etc.

1. ' $\mathrm{X}$ ' icon to extract time histories

\section{$\underline{\text { DIC Coordinate System }}$}

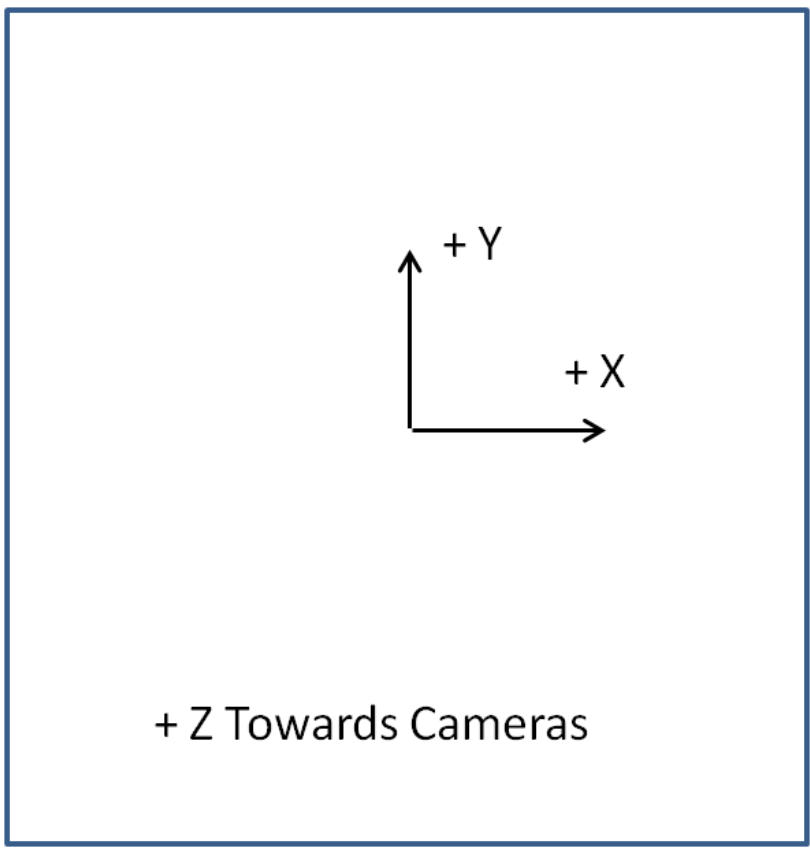




\section{ENERGY ANALYSIS}

This manual is designed for the steps to use the energy and impulse analysis code.

(1) Obtain the original data

The original data of shock tube experiments are from the Tektronix oscilloscope (TDS3014 or 3014C). The data must have following name:

First channel: TEK00000.csv

Second channel: TEK00001.csv

Normally, there are two columns in these files. The unit of the first column is second (s). The unit of the second column is voltage (v).Please copy these files into the folder named "experimental data backup".

(2) Analyze the original data. This step is to analyze the original data to obtain the shock wave velocity, the peak pressure and the modified pressure profiles.

This step is carried out in the folder named "original data analysis". In this folder, the $\mathrm{m}$ file named profile_analysis.m is necessary. Other files can be deleted or replaced. You must copy the original data files into this folder and then run the code. The running process is as follow,

(1) The code will first ask you how many plys you use in the experiment. This information is only for your record. It does not matter the analysis process.

(2) The code will ask you the sensitivity of the sensors. This value is given in the box of the sensors. This value means how many milli-voltage related to 1 psi.

(3) Then, a figure with two plots of the pressure profiles will be given. Look at this figure carefully and determine an approximate biggest time before the first jump time of channel 1. (This time should be less than and as close as possible to the first jump time of channel 1. Normally, should not be farer from the first jump time than 200 microsecond)

(4) Then the code will inform you as follow, 
"The peak and velocity data have been saved into the file, which is named peak\&velocity.txt and in the same folder of this code.

There are two more pressure data files in this folder:

inc_sp.dat

ref_sp.dat

They can be used for energy and impulse evaluation.

The code will give some plots to verify your data.

Please double check them very carefully.

press any key to continue"

(5) After pressing any key, the code will give four images:

Figure 1 Original Data

Figure 2 Modified Pressure Profiles

Figure 3 Pressure profile of Channel 1 with key point marks

Figure 4 Pressure profile of Channel 2 with key point marks

Look at these figures very carefully, especially Figure 3 and 4. If the jump points and peak points are not right, you may need to manually pick up the jump points and peak points.

(6) There will be three saved files in the same folder,

inc_sp.dat

ref_sp.dat

peak\&velocity.txt

The first two files will be used to analyze the energy and impulse. There are two column data in these two files. The first column is time with unit second (s). The second column is pressure data with unit psi.

The last file records the physical parameters, which needs to be input in the energy and impulse analysis.

(7) Please cut these three data files into the folder named "experimental data backup" and delete all of these files in the current folder.

(3) Analyze the incident and remaining energy and impulse 
This step is to use the data obtained in step 2 to analyze the incident and remaining energy and impulse in a shock tube experiment.

This step is carried out in the folder named "gas energy and impulse analysis". In this folder, seven $\mathrm{m}$ files are necessary. They are,

Main code: energy_impulse_analysis.m

Function 1: load_data.m

Function 2: skip_points.m

Function 3: density_change.m

Function 4: sound_speed.m

Function 5: velocity_change.m

Function 6: spline_integ.m

Other files can be deleted or replaced. You must copy the data files: inc_sp.dat and ref_sp.dat, into this folder and the correlated blank test data file, inc.dat, from the folder named "blank test data". The code running process is as follow,

(1) The code will first show the format and unit of the data. Please be sure that the data should be the exact format.

(2) Then the code will give the total number of the data and ask you how many points you want to skip. This means if the original data is

Original Data

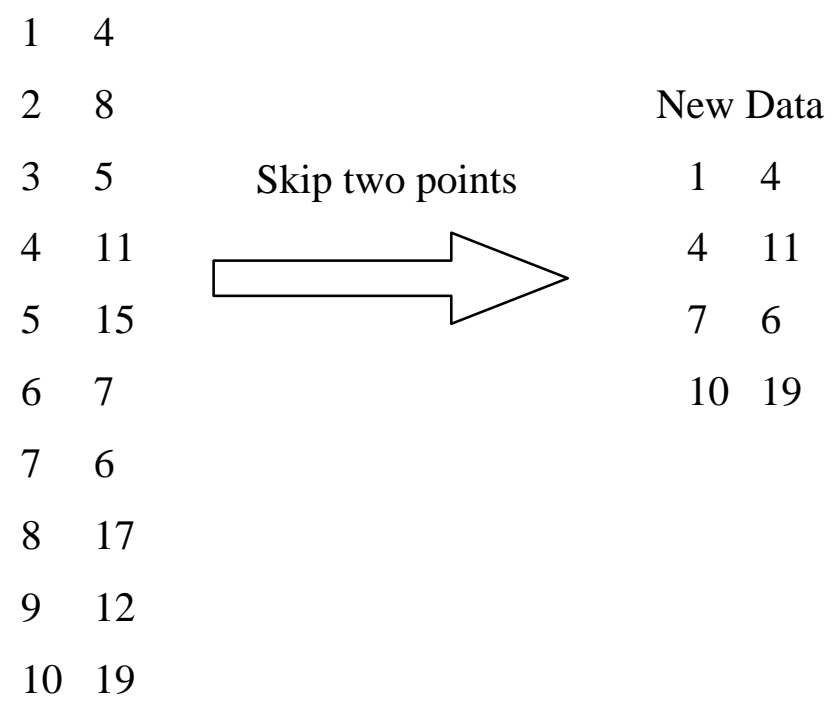


(3) Then the code will ask you to input physical parameters obtained in step 2 (saved in peak\&velocity.txt).

(4) The code will calculate the physical parameter profiles. This process is automatic.

(5) Then the code will integrate the parameter profiles to obtain the energy components. This process is automatic.

(6) Finally, the code will ask you to input the name of the file which you want to save data into. Then all of the data will be saved into filename_gas_energy.mat. The energy and impulse components are saved into ten files as follow,

\begin{tabular}{|c|c|}
\hline filename_incident_internal_E.dat & incident internal energy \\
\hline filename_remaining_internal_E.dat & remaining internal energy \\
\hline filename_incident_translational_E.dat & incident translation energy \\
\hline filename_remaining_translational_E.dat & remaining translational energy \\
\hline filename_incident_work_E.dat & work done by the incident gas \\
\hline filename_remaining_work_E.dat & $\begin{array}{l}\text { work done by the gas behind the } \\
\text { reflected shock wave }\end{array}$ \\
\hline filename_total_incident_E.dat & total incident energy \\
\hline filename_total_remaining_E.dat & total remaining energy \\
\hline filename_total_energy_loss.dat & $\begin{array}{l}\text { absolute difference between the total } \\
\text { incident and remaining energies }\end{array}$ \\
\hline lename_total_impulse.dat & total impulse \\
\hline
\end{tabular}

Please copy and save these data into a safe folder.

(4) Analyze the deformation energy of the gas, momentum and kinetic energy of the specimen

This step is to use the data obtained in step 2 and the high-speed side-view images to analyze the deformation energy of the gas, momentum and kinetic energy of the specimen in a shock tube experiment. 
This step is carried out in the folder named "specimen momentum and energy". In this folder, only one m files, Deformation_momentum_kinetic_photron.m, are necessary. Other files can be deleted or replaced. You must copy the data files: ref_sp.dat, and a series of high-speed images into this folder. The code running process is as follow,

(1) The code will first show the format and unit of the data. Please be sure that the data should be the exact format.

(2) load the time series of the images

You will have three ways to load the time series of the images.

(i) The time between two frames is same. You can input total number of frames and time between two frames. Then the code will generate the time series automatically.

(ii) The time between two frames is not same. You can input total number of frames and input time between two frames frame by frame.

(iii) The time between two frames is not same. The time between two frames is not same. Then you can just load that time series data file. You can choose anyone and following the instruction.

(3) Length calibration. You can choose any image for length calibration. You will need to choose two points on this image and the vertical distance between these two points will be used to calibrate the length. Therefore, you need to know one real vertical scale between two points on the image. For example:

(i) the span of the supports is 6 inches

(ii) the outer diameter of the shock tube is 5 inches

The process will repeat three times. Thus, totally you will pick six times. Please follow the instruction.

(4) Then you can do real measurement. Normally, you need to measure the deformation shape of the front face for every image. For each image, you need to choose seven points on the front face. The code will let you pick up the top point of the front face first. Then it will let you pick up the bottom point of the front face. After this, the code will base on these two points to 
draw seven lines with equivalent distance. Please pick up the points from the top to the bottom.

(5) The code will ask you to input the mass of the specimen.

(6) The code will ask you to input the name of the file which you want to save you data into. 


\section{APPENDIX C}

\section{SAFETY GUIDELINES FOR EXPERIMENTAL EQUIPMENT}

\section{SHPB}

1. Never perform experiment without the help of other students

a. For ease of conducting a safe and efficient experiment

2. Make sure proper precautions are made prior to experiment

a. Wear safety glasses at al 1 times

b. Long sleeve shirts and shoes should be worn, no open toe shoes or sandals

c. Make sure all wires and gauges are adhered correctly and prepared properly

3. Make sure proper bars are being used depending upon specific materials being tested (steel or aluminum bars)
a. Solid-Solid Bar (hard materials)
b. Solid-Hollow Bar (soft materials)
c. Hollow-Hollow (real soft materials)

\section{DO NOT PRESSURIZE GUN UNTIL YOU ARE ABOUT TO FIRE}

a. Do not put fingers between bars, i.e. striker and incident bar or incident and transmitter bar when SHPB is pressurized

b. Do not stand in front of muzzle or try to load striker bar when pressurized

c. Do not leave bar unattended after pressurized

d. If adjustments are needed, vent the pressure beforehand

5. Conduct yourself in a mature and responsible manner at all times in the laboratory

6. Make sure to yell "firing" when experiment is about to be run and SHPB is being pressurized, keep outside doors closed so no one walks in

7. Make sure everyone in the lab, helping or not with the experiment, is aware an experiment will be taking place 


\section{SHOCK TUBE}

1. Do not stand near shock tube when it is being fired

a. Stand no closer than 30 feet in any direction

b. Stand next to person who controls Helium Tank

2. Wear ear and eye protection

3. Warn everyone around you that shock tube is to be fired

a. Make sure Fluids Labs and Microfluidics labs know

b. Make sure no classes are going on in Design Studio

c. Make sure Design Studio students are not there

i. If Design Studio is occupied, and no class is going on, ask students to leave for the test

4. Double check to make sure no one is in specimen lab

5. Check to make sure door to outside is closed and locked

6. Have the shock tube area roped off prior to test

7. Place "warning sign" in front of Microfluidics lab door and close divider off to design studio

8. Be sure to yell "firing" when releasing gas and also around the pressure where the diaphragm is expected to burst

9. Make sure bolts are completely tightened in the enclosure at end of shock tube.

10. Make sure all lexan is in place and both sliding doors are closed

11. Attach vacuum if sample that is to be blasted will cause excessive debris

12. If for any reason someone comes within 30 feet of tube shut off gas immediately and ask person to leave

\section{Procedure For Running a Safe Shock Tube Experiment}

1. Load up cart in DPML lab with proper equipment that is to be used

a. Oscilloscope, High-speed Camera, proper cables, extension cords, duct tape, research notebook, pressure sensors, mylar sheets, etc

b. Ask someone to help you move the cart and camera 
i. Under no circumstance is the High-speed Camera to be moved by less than 2 people.

ii. This includes loading and unloading the camera

2. Enter Shock Tube area through garage door and place cart in position

3. Set up Camera in proper position

4. Set up flash bulbs and/or proper lights

5. Attach cables to necessary components and to triggers

6. Once High-speed Camera and components are set up, then place sample in enclosure

a. Use rubber band for positioning and then tape the sample in place

b. Adjust camera for experiment and focus on sample

7. Set up computer software (Imacon200, or Photron SA1)

8. Trigger camera and adjust images accordingly

9. Once Camera and computer are in place, properly set up oscilloscope place pressure sensors in the shock tube

10. Make sure everything is turned on and set up properly for given test

a. Camera images and triggers for both oscilloscope and camera are dependent on the number of plies being used

11. Make sure mylar is in place between driver and driven section

12. Once mylar is in place and both the camera and oscilloscope are set up properly, double check sample and make sure it is in place and shock tube muzzle is touching it.

13. Check everything over one more time and make sure nothing triggered prematurely

14. Make sure tank is completely shut and follow the safety rules

15. If sample is known to make mess attach vacuum to enclosure tank Go to helium tank and slowly begin releasing gas into the shock tube

16. Remember to yell "firing" when loading and when burst is expected

17. Once sample has been blasted, shut off gas and proceed to checking the scope and camera for data

18. Make sure to save data to respected positions 
a. Camera data saved under "users"

b. Oscilloscope data saved on floppy and then on your computer

i. Double check to make sure data is there

19. Remove sample from shock tube and piece back together

a. Once the piece pertaining to the sample have been collected and the sample put back together as well as possible, take pictures

i. Remember the longer you wait the more time the sample has to creep back and lose shape...which means losing crucial information 


\section{APPENDIX D}

\section{ASTM STANDARDS:}

\section{$\underline{\text { ASTM D 3039/D 3039M - } 08}$}

\section{$\underline{\text { Standard Test Method for Tensile Properties of Polymer Matrix Composite Materials }}$}

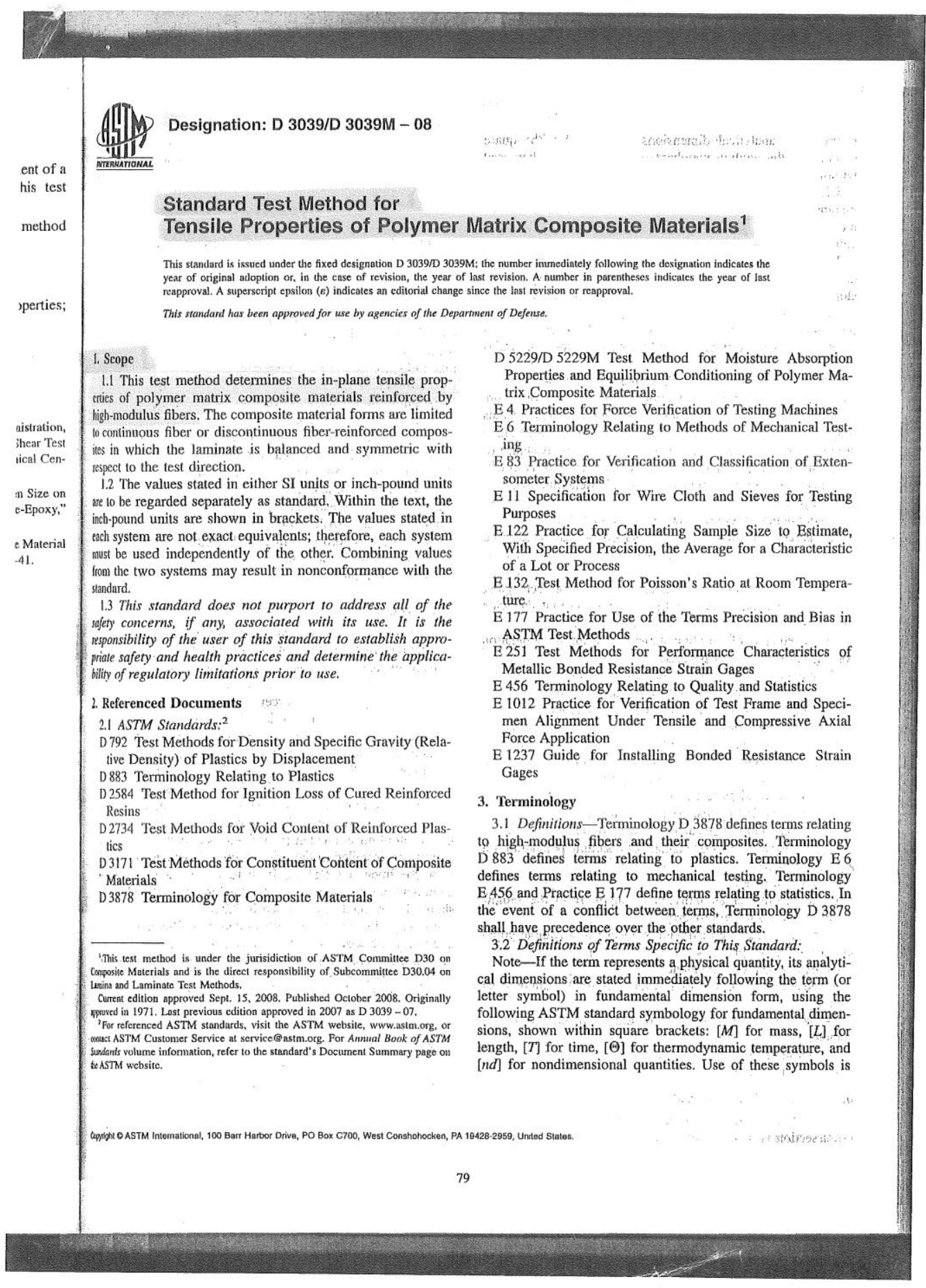


$\underline{\text { ASTM D 3410/D 3410M - } 03}$

\section{$\underline{\text { Standard Test Method for Compressive Properties of Polymer Matrix Composite }}$}

\section{Materials with Unsupported Gage Section by Shear Loading}

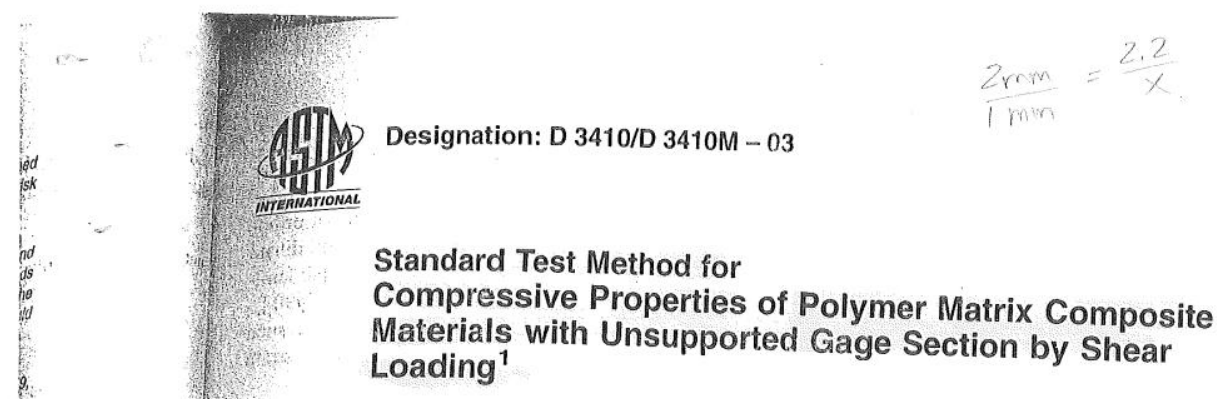

This standard is issued under the fixed designation D $3410 / \mathrm{D} 3410 \mathrm{M}$; the number immediately following the designation indicates the year of original adoption or, in the case of revision, the year of last revision. A number in parentheses indicates the year of last
reapproval. A superscript epsilon ( $\epsilon$ ) indicates an editorial change Thistard the last revision or reapproval.

This standard has been approved for use by agencies of the Department of Defense.

\section{Scope}

1.1 This test method determines the in-plane compressive properties of polymer matrix composite materials reinforced by high-modulus fibers. The composite material forms are limited to continuous-fiber or discontinuous-fiber reinforced composites for which the elastic properties are specially orthotropic with respect to the test direction. This test procedure introduces the compressive force into the specimen through shear at wedge grip interfaces. This type of force tronger at the procedure in Test Method D 695 where compressive force is transmitted into the specimen by end-loading, Test Method D 6641/D 6641M where compressive force is transmitted by combined shear and end loading, and Test Method D 5467/ D $5467 \mathrm{M}$ where compressive force is transmitted by subjecting a honeycomb core sandwich beam with thin skins to four-point

1.2 This test method is applicable to composites made from unidirectional tape, wet-tow placement, textile (for example, fabric), short fibers, or similar product forms. Some product forms may require deviations from the test method.

1.3 The values stated in either SI units or inch-pound units are to be regarded separately as standard. Within the text the inch-pounds tnits are shown in brackets. The values stated in each system are not exact equivalents; therefore, each system must be used independently of the other. Combining values from the two systems may result in nonconformance with the standard.

Nотв 1-Additional procedures for determining compressive properties of resin-matrix composites may be found in Test Methods D 695 ,

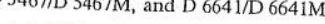

1.4. This standard does not purport to address all of the safety concerns, if any, associated with its use. It is the responsibility of the user of this standard to establish appro-

'This specification is under the jurisdiction of ASTM Committee D30 Composite Materials and is the direct responsibility of Subcommittee D30.04 on Lamina and Laminate Test Methods.

Current edition approved June 10, 2003. Published August 2003. Originly approved in 1975. Last previous edition approved in 1995 as D 3410/D 3410M - 95. priate safety and health practices and determine the applica bility of regulatory limitations prior to use.

2. Referenced Documents

2.1 ASTM Standards:

D 695 Test Method for Compressive Properties of Rigid Plastics $^{2}$

D 792 Test Methods for Density and Specific Gravity (Relative Density) of Plastics by Displacement ${ }^{2}$

D 883 Terminology Relating to Plastics ${ }^{2}$

D 2584 Test Method for Ignition Loss of Cured Reinforced Resins $^{3}$

D 2734 Test Methods for Void Content of Reinforced Plastics $^{3}$

D 3171 Test Method for Constituent Content of Composite Materials $^{4}$

D 3878 Terminology for Composite Materials ${ }^{4}$

D 5229/D 5229M Test Method for Moisture Absorption Properties and Equilibrium Conditioning of Polymer Matrix Composite Materials ${ }^{4}$

D 5379/D 5379M Test Method for Shear Properties of Composite Materials by the V-Notched Beam Method ${ }^{4}$ D 5467/D 5467M Test Method for Compressive Properties of Unidirectional Polymer Matrix Composites Using Sandwich Beam ${ }^{4}$

D 6641/D 6641M Test Method for Determining the Compressive Properties of Polymer Matrix Composite Laminates Using a Combined Loading Compression (CLC) Test Fixture $^{4}$

E 4 Practices for Force Verification of Testing Machines 5 E 6 Terminology Relating to Methods of Mechanical Testing $^{5}$

E 83 Practice for Verification and Classification of Extensometers $^{5}$

E 111 Test Method for Young's Modulus, Tangent Modulus,

${ }^{2}$ Annual Book of ASTM Standards, Vol 08.01.

${ }^{3}$ Annual Book of ASTM Standards, Vol 08.02 .

${ }_{5}^{4}$ Annual Book of ASTM Standards, Vol 15,03.

${ }^{5}$ Annual Book of ASTM Standards, Vol 03.01 .

Copyright O ASTM International, 100 Barr Harbor Drive, PO Box C700, West Conshohocken, PA 19428-2959, United States. 
$\underline{\text { ASTM D } 1621-04 a}$

\section{$\underline{\text { Standard Test Method for Compressive Properties of Rigid Cellular Plastics }}$}

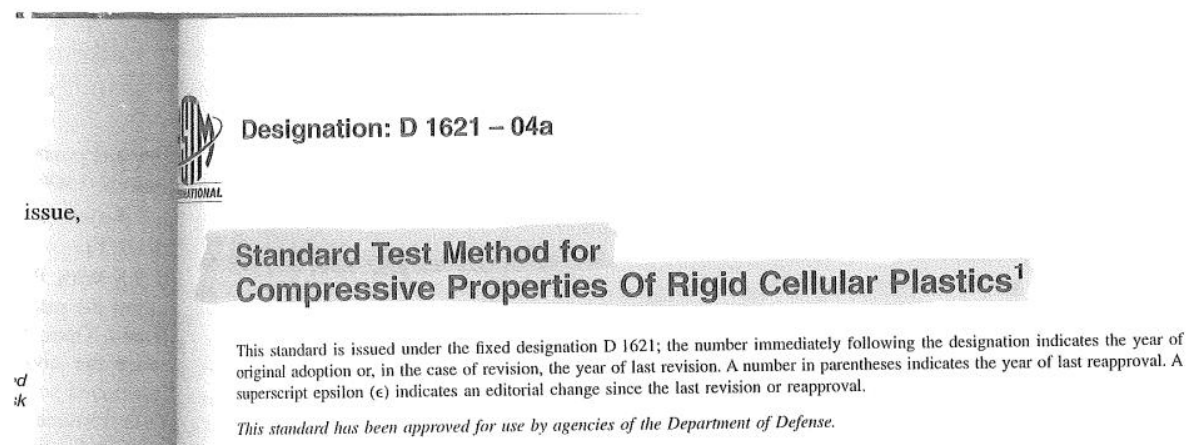

This test method describes a procedure for determining this test method describes a properties of rigid cellular materials, particuexpanded plastics.

The values stated in SI units are to be regarded as the

a. The values in brackets are for information only.

a This standard does not purport to address all of the concerns, if any, associated with its use. It is the wibility of the user of this standard to establish approssfety and health practices and determine the applicaof regulatory limitations prior to use.

- T-This test method and ISO 844 are technically equivalent.

8ferenced Documents

4. ISTM Standards: ${ }^{2}$

618 Practice for Conditioning Plastics for Testing

4 Practices for Force Verification of Testing Machines

83 Practice for Verification and Classification of Exten-

inteter Systems

691 Practice for Conducting an Interlaboratory Study to

Dtermine the Precision of a Test Method

i. 150 Standard:

0844 Cellular Plastics - Compression Test of Rigid Masinals $s^{3}$

Irminology

41 Definitions:

III conpliance - the displacement difference between test ine drive system displacement values and actual specimen trement.

The Fists melhod is under the jurisdiction of ASTM Committee D20 on Plics.

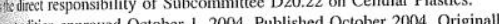
antedition approved October 1,2004 . Published October 2004. Ori in in 1959. Last previous edition approved in 2004 as D 1621 - 04.

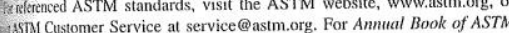
istance information, refer to the standard's Document Summary page on a) website.

thistle from American National Standards Institute (ANSI), 25 W. 43rd St.

3.1 .2 compliance correction - an analytical method of modifying test instrument displacement values to eliminate the amount of that measurement attributed to test instrument compliance.

3.1.3 compressive deformation - the decrease in length produced in the gage length of the test specimen by a compressive load expressed in units of length.

3.1.4 compressive strain - the dimensionless ratio of compressive deformation to the gage length of the test specimen or the change in length per unit of original length along the longitudinal axis.

3.1 .5 compressive strength - the stress at the yield point if a yield point occurs before $10 \%$ deformation (as in Fig. 1a) or, in the absence of such a yield point, the stress at $10 \%$ deformation (as in Fig. 1b)

3.1.6 compressive stress (nominal) - the compressive load per unit area of minimum original cross section within the gage boundaries, carried by the test specimen at any given moment, expressed in force per unit area.

3.17 compressive stress-strain diagram-a diagram in which values of compressive stress are plotted as ordinates against corresponding values of compressive strain as abscis sas.

3.1 .8 compressive yield point-the first point on the stressstrain diagram at which an increase in strain occurs without an increase in stress.

3.1 .9 deflectometer - a device used to sense the compressive deflection of the specimen by direct measurement of the distance between the compression platens.

3.1.10 displacement-compression platen movement after the platens contact the specimen, expressed in millimetres or inches.

3.1.11 gage length-the initial measured thickness of the test specimen expressed in units of length.

3.1 .12 modulus of elasticity - the ratio of stress (nominal) to corresponding strain below the proportional limit of a materia expressed in force per unit area based on the minimum initial cross-sectional area.

*A Summary of Changes section appears at the end of this standard. 
$\underline{\text { ASTM D 7136/D 7136M - } 07}$

\section{$\underline{\text { Standard Test Method for Measuring the Damage Resistance of a Fiber-Reinforced }}$}

\section{$\underline{\text { Polymer Matrix Composite to a Drop-Weight Impact Event }}$}

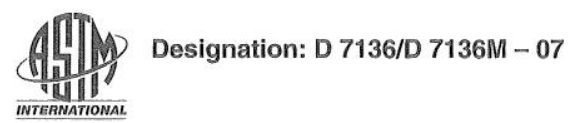

\section{Standard Test Method for Measuring the Damage Resistance of a Fiber-Reinforced Polymer Matrix Composite to a Drop-Weight Impact Event ${ }^{1}$}

This standard is issued under the fixed designation D 7136/D 7136M; the number immediately following the designation indicates the
year of original adoption or, in the case of revision, the year of last revision. A number in parentheses indicates the year of last year of original adoption or, in the case of revision, the year of last revision. A number in parentheses indicates the year of last reapproval. A superscript epsilon $(\varepsilon)$ indicates an editorial change since the last revision or reapproval.

1. Scope

1.1 This test method determines the damage resistance of multidirectional polymer matrix composite laminated plates subjected to a drop-weight impact event. The composite material forms are limited to continuous-fiber reinforced polymer matrix composites, with the range of acceptable test laminates and thicknesses defined in 8.2 .

1.2 A flat, rectangular composite plate is subjected to an out-of-plane, concentrated impact using a drop-weight device with a hemispherical impactor. The potential energy of the drop-weight, as defined by the mass and drop height of the impactor, is specified prior to test. Equipment and procedures are provided for optional measurement of contact force and velocity during the impact event. The damage resistance is quantified in terms of the resulting size and type of damage in the specimen.

1.3 The test method may be used to screen materials for damage resistance, or to inflict damage into a specimen for subsequent damage tolerance testing. When the impacted plate is tested in accordance with Test Method D 7137/D 7137M, the overall test sequence is commonly referred to as the Compression After Impact (CAI) method. Quasi-static indentation per Test Method D 6264 may be used as an alternate method of creating damage from an out-of-plane force and measuring damage resistance properties.

1.4 The damage resistance properties generated by this test method are highly dependent upon several factors, which include specimen geometry, layup, impactor geometry, impactor mass, impact force, impact energy, and boundary conditions. Thus, results are generally not scalable to other configurations, and are particular to the combination of geometric and physical conditions tested.

1.5 The values stated in either SI units or inch-pound units are to be regarded separately as standard. Within the text the inch-pound units are shown in brackets. The values stated in

'This test method is under the jurisdiction of ASTM Committee D30 on Composite Materials and is the direct responsibility of Subcommittee D30.05 on Structural Test Methods.

Current edition approved Oct. 1, 2007. Published November 2007. Originally approved in 2005. Last previous edition approved in 2005 as D 7136/D 7136M each system are not exact equivalents; therefore, each sys must be used independently of the other. Combining val from the two systems may result in nonconformance with standard.

1.6 This standard does not purport to address all of safety concerns, if any, associated with its use. It is responsibility of the user of this standard to establish ap priate safety and health practices and determine the appl

bility of regulatory limitations prior to use.

2. Referenced Documents

2.1 ASTM Standards: ${ }^{2}$

D 792 Test Methods for Density and Specific Gravity ( $R$ tive Density) of Plastics by Displacement

D 883 Terminology Relating to Plastics

D 3171 Test Methods for Constituent Content of Compe

Materials

D 3763 Test Method for High Speed Puncture Propertie

Plaśtics using Load and Displacement Sensors

D 3878 Terminology for Composite Materials

D 5229/D 5229M Test Method for Moisture Absom

Properties and Equilibrium Conditioning of Polymerl trix Composite Laminates

D 5687/D 5687M Guide for Preparation of Flat Compos

Panels with Processing Guidelines for Specimen Prer tion

D 6264 Test Method for Measuring the Damage Resit of a Fiber-Reinforced Polymer-Matrix Composite 1 Concentrated Quasi-Static Indentation Force

D 7137/D 7137M Test Method for Compressive Res Strength Properties of Damaged Polymer Matrix Con ite Plates

E 4 Practices for Force Verification of Testing Machin E 6 Terminology Relating to Methods of Mechanical ing

E 18 Test Methods for Rockwell Hardness and Rod

ASTM standards, visit the ASTM website, www.astm contact ASTM Customer Service at service@astm.org. For Ammual Book o Standards volume information, refer to the standard's Document Summan? the ASTM website.
MIL-H

ume

Desiz

MIL-HI

MIL-H

ComI

MIL-HI

Comf

MIL-HI

Comp

MIL-HI

Comp

NASA

Tough

$1983^{5}$

3. Termin

3.1 Defir

to compos:

relating to

mechanical

define term

between teI

over the ott.

3.2 Defin

term represi

are stated in

fusdamental

standard sy

within squar

lime, $[\theta]$ for

dimensional

analytical di

symbols ma

brackets.

3.2.1 dent

formed by ar

shall be defin

Available fo en Proving Gro Available fro 
$\underline{\text { ASTM D 7137/D 7137M - } 07}$

\section{$\underline{\text { Standard Test Method for Compressive Residual Strength Properties of Damaged }}$}

\section{$\underline{\text { Polymer Matrix Composite Plates }}$}

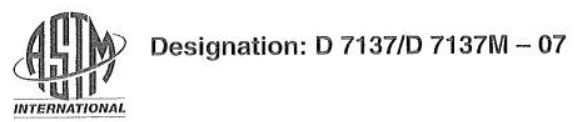

\section{Standard Test Mlethod for Compressive Residual Strength Properties of Damaged Polymer Matrix Composite Plates ${ }^{1}$}

This standard is issued under the fixed designation D 7137/D 7137M; the number immediately following the designation indicates the year of original adoption or, in the case of revision, the year of last revision. A number in parentheses indicales the year of last reapproval. A superscript epsilon $(\epsilon)$ indicates an editorial change since the last revision or reapproval,

\section{Scope}

1.1 This test method covers compression residual strength properties of multidirectional polymer matrix composite laminated plates, which have been subjected to quasi-static indentation per Test Method D 6264/D 6264M or drop-weight impact per Test Method D 7136/D 7136M prior to application of compressive force. The composite material forms are limited to continuous-fiber reinforced polymer matrix composites with multidirectional fiber orientations, and which are both symmetric and balanced with respect to the test direction. The range of acceptable test laminates and thicknesses is defined in 8.2.

NOTE 1-When used to determine the residual strength of drop-weight impacted plates, this test method is commonly referred to as the Compression After Impact, or CAI, method

1.2 The method utilizes a flat, rectangular composite plate, previously subjected to a damaging event, which is tested under compressive loading using a stabilization fixture.

Notr: 2-The damage tolerance properties obtained are particular to the type, geometry and location of damage inflicted upon the plate.

1.3 The properties generated by this test method are highly dependent upon several factors, which include specimen geometry, layup, damage type, damage size, damage location, and boundary conditions. Thus, results are generally not scalable to other configurations, and are particular to the combination of geometric and physical conditions tested.

1.4 This test method can be used to test undamaged polymer matrix composite plates, but historically such tests have demonstrated a relatively high incidence of undesirable failure modes (such as end crushing). Test Method D 6641/D 6641M is recommended for obtaining compressive properties of undamaged polymer matrix composites.

1.5 The values stated in either SI units or inch-pound units are to be regarded separately as standard. Within the text the inch-pound units are shown in brackets. The values stated in

'This test method is under the jurisdiction of ASTM Committee D30 on Composite Materials and is the direct responsibility of Subcommittee D30.05 on Structural Test Methods.

Current edition approved Nov. 15, 2007. Published December 2007. Originally $05^{* 1}$. each system are not exact equivalents; therefore, eat must be used independently of the other. Combinil from the two systems may result in nonconformance standard.

1.6 This standard does not purport to address , safety concerns, if any, associated with its use. responsibility of the user of this standard to establi. priate safety and health practices and determine the bility of regulatory limitations prior to use.

\section{Referenced Documents}

2.1 ASTM Standards: ${ }^{2}$

D 792 Test Methods for Density and Specific Grav tive Density) of Plastics by Displacement D 883 Terminology Relating to Plastics

D 3171 Test Methods for Constituent Content of C Materials

D 3878 Terminology for Composite Materials

D 5229/D 5229M Test Method for Moisture Al

Properties and Equilibrium Conditioning of Poly trix Composite Materials

D 5687/D 5687M Guide for Preparation of Flat C Panels with Processing Guidelines for Specimen tion

D 6264/D 6264M Test Method for Measuring the Resistance of a Fiber-Reinforced Polymer-Mat posite to a Concentrated Quasi-Static Indentation

D 6641/D 664IM Test Method for Determining II pressive Properties of Polymer Matrix Composil nates Using a Combined Loading Compression (C Fixture

D 7136/D 7136M Test Method for Measuring the Resistance of a Fiber-Reinforced Polymer Mato posite to a Drop-Weight Impact Event

E 4 Practices for Force Verification of Testing $M x$ E 6 Terminology Relating to Methods of Mechani ing

${ }^{2}$ For referenced ASTM standards, visit the ASTM website, wwi contact ASTM Customer Service at service@ astm.org. For Amual R Standarls volume information, refer to the standard's Document Sum the ASTM website 


\section{BIBLIOGRAPHY}

Allen, H., "Analysis and design of structural sandwich panels", Pergamon Press, Inc., Oxford, 1969.

Amini, M. R., Amirkhizi, A. V., and Nemat-Nasser, S., "Numerical modeling of response of monolithic and bilayer plates to impulsive loading", International Journal of Impact Engineering, 37(2010) 90-102

Amini, M. R., Isaacs, J. B., and Nemat-Nasser S., "Experimental investigation of response of monolithic and bilayer plates to impulsive loading", International Journal of Impact Engineering, 37(2010) 82-89

Amini, M. R., Isaacs, J. B., and Nemat-Nasser, S.., "Investigation of effect of polyurea on response of steel plates to impulsive loads in direct pressure-pulse experiments", Mechanics of Materials, 42 (2010) 628-639

Amini, M. R., Isaacs J. B., and Nemat-Nasser, S., "Numerical modeling of effect of polyurea on response of steel plates to impulsive loads in direct pressure-pulse experiments", Mechanics of Materials, 42 (2010) 615-627

Amirkhizi, A. V., Isaacs, J., McGee, J., and Nemat-Nasser, S., “An experimentallybased constitutive model for polyurea, including pressure and temperature effects”, Philosophical Magazine, 86 (36): (2006) 5847-5866

Apetre, N.A., Sankar, B.V. and Ambur, D.R., "Low-velocity impact response of sandwich beams with functionally graded core", International Journal of Solids and Structures, 43 (2006) 2479-2496. 
Atas, C., and Sevim, C., "On the impact response of sandwich composites with cores of balsa wood and PVC foam”, Composite Structures, 93 (2010) 40-48.

Bahei-El-Din, Y. A., Dvorak, G. J., and Fredricksen, O.J., “A blast-tolerant sandwich plate design with a polyurea interlayer", International Journal of Solids and Structures, 43 (2006) 7644-7658

Becu, L., Maazouz, A., Sautereau, H., and Gerard JF, "Fracture behavior of epoxy polymers modified with core-shell rubber particles", Journal of Applied Polymer Science, 65 (1997) 2419-2431.

Broutman, L. J., and Sahu, S., "The effect of interfacial bonding on the toughness of glass filled polymers”, Material Sciences and Engineering, 8 (1971) 98-107.

Bruck, H., "A one-dimensional model for designing functionally graded materials to manage stress waves", International Journal of Solids and Structures, 37 (2000) 6383-6395.

Bucknall, C. B., and Partridge, I. K., "Phase separation in epoxy resins containing polyethersulphone", Polymer, 24 (1983) 639 - 644

Carlsson, L. A., and Kardomateas, G. A., "Structural and failure mechanics of sandwich composites”, Springer, New York, 2011.

Changjing, X., Yongxiang, D., Liping, C., Dongjie, X., and Shunhong, W., "Propagation characteristic of explosive waves in layered media: Numerical analysis”, Defence Science Journal, 59 (2009) 499-504.

Chen, W., Zhang, B., and Forrestal, M.J., “A split Hopkinson bar technique for lowimpedance materials", Experimental Mechanics, 39 (1998) 81-85. 
Chiras, S., Mumm, D., Evans, A., Wicks, N., Hutchinson, J., Dharmasena, H., Wadley, H., and Fichter, S., "The structural performance of near-optimized truss core panels", International Journal of Solids and Structures, 39 (2002) 4093-4115.

Cook, J., and Walsh, S., "Enhancing the Vacuum Assisted Resin Transfer Molding (VARTM) process through the alternation of variables", Army Research Laboratory, 2003.

Corecell $^{\mathrm{TM}}$ A-Series Foam Material Data Sheet. www.gurit.com (Accessed April 3, 2012)

Courant, R., and Friedrichs, K.O., "Supersonic flow and shock waves", Interscience Publishers, New York, 1948.

Cui, L., Kiernan, S., Gilchrist, M. D., "Designing the energy absorption capacity of functionally graded foam materials", Materials Science and Engineering: A, 507 (2009) 215-225.

Derakane 411-200 Material Data Sheet, www.ashland.com (Accessed April 3, 2012)

Derakane 8084 Material Data Sheet, www.ashland.com (Accessed April 3, 2012)

Dharmasena, K.P., Wadley, H.N.G., Xue, Z. and Hutchinson, J.W., "Mechanical response of metallic honeycomb sandwich panel structures to high-intensity dynamic loading”, International Journal of Impact Engineering, 35 (2008) 1063-1074.

Dixon, D. G., Harris, S. J., Dempster, M., and Nicholls, P., "Effect of PEEK fibers and powder on joints made with high temperature adhesive", Journal of Adhesion, 65 (1998) 131-162. 
Dragonshield-HT Polyurea Material Data Sheet. www.specialty-products.com (Accessed April 3, 2012)

Fleck, N.A., Deshpande, V.S., "The resistance of clamped sandwich beams to shock loading”, Journal of Applied Mechanics, 71 (2004) 386-401.

Gardner, N., Wang, E., Kumar, P., and Shukla, A., "Blast mitigation in a sandwich composite using graded core with polyurea interlayer", Experimental Mechanics, 2011. DOI 10.1007/s11340-011-9517-9.

Gardner, N., Wang, E., and Shukla A., "Performance of functionally graded sandwich beams under shock wave loading”, Composite Structures, 2011. DOI 10.1016/j.compstruct.2011.12.006.

Giannakopoulos, G., Masania, K., and Taylor, A. C., "Toughening of epoxy using core-shell particles”, Journal of Material Science, 46 (2011) 327-338.

Gibson, L. J., and Ashby, M. F., "Cellular Solids: Structures and Properties", Elmsford, NY: Pergamon Press, Inc., 1988.

Griffiths, R., and Holloway, D., "The fracture energy of some epoxy resin materials", Journal of Materials and Sciences, 5 (1970) 302-307.

Hoo Fatt, M. S., Ouyang, X., and Dinan, R. J., "Blast response of walls retrofitted with elastomer coatings", Journal of Structural Materials, 15 (2004) 129-138.

Hossain, M. K., Liu, Q. L., and O’Toole, B. J., "Functionally graded foam material system for energy absorption", SAMPE $39^{\text {th }}$ ISTC-Cincinnati OH, Oct. 29 Nov. 1, 2007.

Hsieh, T. H., Kinloch, A. J., Masania, K., Sohn Lee, J., Taylor, A. C., and Sprenger, S., "The toughness of epoxy polymers and fiber composites modified with 
rubber microparticles and silica nanoparticles", Journal of Materials and Sciences, 45 (2010) $1193-1210$.

Johnsen, B. B., Kinloch, A. J., Taylor, A. C., "Toughness of syndiotactic polystyrene/epoxy polymer blends: microstructure and toughening mechanisms", Polymer, 46 (2005) 7352-7369.

Kaneka Kane Ace MX 153Material Data Sheet, http://www.kanekatexas.com (Accessed April 3, 2012)

Kaneka Texas Corporation, http://www.kanekatexas.com/MX.html (Accessed April 3, 2012)

Kiernan, S., Cui, L., and Gilchrist, M. D., "Propagation of a stress wave through a virtual functionally graded foam", International Journal of Non-Linear Mechanics, 44 (2009) 456-468.

Kinloch, A. J., Yuen, M. L., and Jenkins, S. D., "Thermoplastic-toughened epoxy polymers", Journal of Material Science, 29 (1994) 3781-3790.

Kinloch, A. J., Mohammed, R. D., Taylor, A. C., Sprenger, S., and Egan, D., "The interlaminar toughness of carbon-fiber reinforced plastic composites using 'hybrid-toughened' matrices", Journal of Material Science, 41(2006) 50435046.

Kinloch, A. J., Shaw, S. J., Tod, D. A., Hunston, D. L., "Deformation and fracture behavior of a rubber-toughened epoxy: 1. Microstructure and fracture studies", Polymer, 24 (1983) 1341- 1354. 
Kinloch, A. J., and Taylor, A. C., "The toughening of cryanate-ester polymers -Part I - Physical modification using particles, fibers and woven mats”, Journal of Materials and Sciences, 37 (2002) 433-460.

Kumar, P., LeBlanc, J., Stargel, D., and Shukla, A., "Effect of plate curvature on blast response of aluminum panels", Impact Engineering, (2012) Accepted

LeBlanc, J., Shukla, A., Rousseau, C., and Bogdanovich, A., "Shock loading of threedimensional woven composite materials”, Composite Structures, 79, (2007) 344- 355 .

Li, R.F., Kardomateas, G.A. and Simitses, G.J., "Nonlinear Response of a Shallow: Sandwich Shell With Compressible Core to Blast Loading”, Journal of Applied Mechanics-Transactions of the ASME, 75 (2008) 125-138.

Li, Y., Ramesh, K.T. and Chin, E.S.C., "Dynamic characterization of layered and graded structures under impulsive loading”, International Journal of Solids and Structures, 38 (2001) 6045-6061.

Liang, Y., Spuskanyuk, A. V., Flores, S. E., Hayhurst, D. R., Hutchinson, J. W., McMeeking, R. M., and Evans, A. G., "The response of metallic sandwich panels to water blast”, Journal of Applied Mechanics, 74 (2007) 81-99.

Lim, T. S., Lee, C. S., and Lee, D. G., "Failure modes of foam core sandwich beams under static and impact loads", Journal of Composite Materials, 38 (2002) 1639-1662.

Lin, K. F., and Shieh, Y. D., "Core-Shell Particles Designed for Toughening the Epoxy Resins: I. Preparation and Characterization of Core-Shell Particles", Journal of Applied Polymer Science, 69 (1998) 2069-2078. 
Lin, K. F., and Shieh, Y. D., "Core-shell particles designed for toughening the epoxy resins: II. Core-shell particle toughened epoxy resins", Journal of Applied Polymer Science, 70 (1998) 2313 -2322.

Makris, A., Frost, D. L., Nerenberg, J., and Lee, J. H. S., "Attenuation of a blast wave with a cellular material", In: Sturtevant B, Shepard JE, Hornung HG, editors. Proceedings of the $20^{\text {th }}$ International Symposium on Shock Waves (ISSW/20), Pasadena, CA, World Scientific. Vol. II, (1996) 1387-1392.

McGrath, L. M., Parnas, R. S., Lenhart, J. L., and King, S., "Fracture toughness of alumina-epoxy composites", Polymer Material Sciences and Engineering, Preprints, 94 (2006) $683-684$.

McShane, G. J., Deshpande, V. S., and Fleck, N. A., "The underwater blast resistance of metallic sandwich beams with prismatic lattice cores", Journal of Applied Mechanics,74 (2007) 352-364.

McShane, G.J., Radford, D.D., Deshpande, V.S. and Fleck, N.A., "The response of clamped sandwich plates with lattice cores subjected to shock loading", European Journal of Mechanics - A: Solids, 25 (2006) 215-229.

Nerenberg, J., Nemes, J. A., Frost, D. L., and Makris, A., "Blast wave loading of polymeric foam", In: Houwing AFP et al., editors. Proceedings of the $21^{\text {st }}$ International Symposium on Shock Waves (ISSW/21), Australia, Panther Publishing and Printing, Vol. I, (1997) 91-96.

Nurick, G.N., Langdon, G.S., Chi, Y. and Jacob, N., “ Behavior of sandwich panels subjected to intense air blast: part 1- Experiments", Composite Structures, (2008), in press. 
Perl R., “Terrorism: US responses to bombings in Kenya and Tanzania: A new policy direction?", Congressional Report. Congressional Research Service, The Library of Congress (1998).

Perl, R., and O'Rourke, R., “Terrorist attack on USS Cole: Background and issues for Congress. Emerging Technologies: Recommendations for counter-terrorism” Institute for Security Technology Studies, Dartmouth College (2001)

Petel, O. E., Jette, F. X., Goroshin, S., Frost, D. L., and Ouellet, S., "Blast wave attenuation through a composite of varying layer distribution", Shock Waves, (2011). DOI 10.1007/s00193-010-0295-6.

Radford, D. D., Fleck, N. A., and Deshpande, V. S., "The response of clamped sandwich beams subjected to shock loading", International Journal of Impact Engineering, 32 (2004) 968-987.

Radford, D.D., McShane, G.J., Deshpande, V.S. and Fleck, N.A., “The response of clamped sandwich plates with metallic foam cores to simulated blast loading”, International Journal of Solids and Structures, 44 (2006) 6101-6123.

Roland, C. M., Twigg, J. N., Vu, Y., and Mott, P. H., "High strain rate mechanical behavior of polyurea", Polymer, 48 (2006) 574-578.

Samadhiya, R., Mukherjee, A., and Schmauder, S., "Characterization of discretely graded materials using acoustic wave propagation", Computational Materials Science, 37 (2006) 20-28.

Spanoudakis, J., Young, R. J., "Crack propagation in a glass particle- filled epoxy resin - Part 1", Journal of Materials and Sciences, 19 (1984) 473-486. 
Steeves, C. A., and Fleck, N. A., "Collapse mechanisms of sandwich beams with composite facesheets and a foam core, loaded in three-point bending. Part I: analytical models and minimum weight design", International Journal of Mechanical Sciences, 46 (2004) 561-583.

Steeves, C. A., and Fleck, N. A., "Collapse mechanisms of sandwich beams with composite facesheets and a foam core, loaded in three-point bending. Part II; experimental investigation and numerical modeling", International Journal of Mechanical Sciences, 46 (2004) 585-608.

Tagarielli, V. L., Deshpande, V. S., and Fleck, N. A., "The high strain rate response of PVC foams and end-grain balsa wood", Composites: Part B, 39 (2008) 83-91.

Tekalur, S. A., Shukla, A., and Shivakumar, K., "Blast resistance of polyurea based layered composite materials", Composite Structures, 84 (2008) 271-281.

Tekalur, S.A., Bogdanovich, A.E. and Shukla, A., "Shock loading response of sandwich panels with 3-D woven E-glass composite skins and stitched foam core", Composite Science and Technology (2008), doi:10.1016/j.compscitech.2008.03.017.

Theotokoglou, E., "Analytical determination of the ultimate strength of sandwich beams", Applied Composite Materials, 3 (1996) 354-353.

Tilbrook, M. T., Deshpande, V. S., and Fleck, N. A., "The impulsive response of sandwich beams: Analytical and numerical investigation of regimes of behavior", Journal of Mechanics and Physics of Solids, 54 (2006) 2242-2280. 
Tiwari, V., Sutton, M. A., and McNeill, S. R., “Assessment of High-speed Imaging Systems for 2D and 3D Deformation Measurements: Methodology Development and Validation”, Experimental Mechanics, 47 (2007) 561-579.

Tiwari, V., Sutton, M. A., McNeill, S. R., Xu, S., Deng, X., Fourney, W.L., Bretall, D., "Application of 3D Image Correlation for Full-Field Transient Plate Deformation Measurements During Blast Loading”, International Journal of Impact Engineering, 36 (2009) 862-874.

Wadley, H.N.G., Dharmasena, K.P., Chen, Y., Dudt, P., Knight, D., Charette, R. and Kiddy, K., "Compressive response of multilayered pyramidal lattices during underwater shock loading", International Journal of Impact Engineering, 35 (2008) 1102-1114.

Wang, E., "Blast resistance and energy mitigation behavior of sandwich composite materials", Ph.D Dissertation, University of Rhode Island, 2010.

Wang, E., and Shukla, A., "Analytical and experimental evaluation of energies during shock wave loading”, International Journal of Impact Engineering, 37 (2010) 1188-1196.

Wang, E., Gardner, N., and Shukla, A., "The blast resistance of sandwich composites with stepwise graded cores", International Journal of Solids and Structures, 46 (2009) 3492-3502.

Wang, L. L., "Foundation of stress waves”, Elsevier, 2007.

Wang, Z., Jing, L., Ning, J., and Zhao, L., "The structural response of clamped sandwich beams subjected to impact loading", Composite Structures, 93 (2011) 1300-1308. 
Wright, J., Shock tubes. John Wiley and Sons Inc, New York, 1961.

Wu, H. T., and Springer, G. S., "Impact induced stresses, strains, and delaminations caused by impact on composite plates”, Journal of Composite Materials, 22 (2008) $533-560$.

Xue, Z. and Hutchinson, J.W., Preliminary assessment of sandwich plates subject to blast loads, International Journal of Mechanical Sciences, 45 (2003) 687-705.

Yan, C., Xiao, K. Q., Ye, L., and Mai, Y. W. "Numerical and experimental studies on the fracture behavior of rubber toughened epoxy in bulk specimen and laminated composites”, Journal of Material Science, 37 (2002) 921-927.

Yee, A. F., and Pearson, R. A., "Toughening mechanisms in elastomer-modified epoxies", Journal of Material Science, 21 (1986) 2462-2474.

Yi, J., Boyce, M. C., Lee, G. F., and Balizer, E., "Large deformation rate-dependent stress-strain behavior of polyurea and polyurethanes", Polymer, 47 (2005) 319329.

Zhu, F., Zhao, L., Lu, G. and Wang, Z., "Deformation and failure of blast loaded metallic sandwich panels - Experimental investigations", International Journal of Impact Engineering, 35 (2008) 937-951. 